
Digital Media Practices in Households 


\section{MediaMatters}

MediaMatters is an international book series published by Amsterdam University Press on current debates about media technology and its extended practices (cultural, social, political, spatial, aesthetic, artistic). The series focuses on critical analysis and theory, exploring the entanglements of materiality and performativity in 'old' and 'new' media and seeks contributions that engage with today's (digital) media culture.

For more information about the series see: www.aup.nl 


\title{
Digital Media Practices in Households
}

\author{
Kinship through Data
}

Larissa Hjorth, Kana Ohashi, Jolynna Sinanan, Heather Horst, Sarah Pink, Fumitoshi Kato and Baohua Zhou 
This project has received funding from the Australian Research Council Linkage scheme. The project, entitled Locating the Mobile, was in partnership with Intel and Distinguished Professor Genevieve Bell.

Cover illustration: Photo by Jolynna Sinanan

Cover design: Coördesign, Leiden

Lay-out: Crius Group, Hulshout

$\begin{array}{ll}\text { ISBN } & 9789462989504 \\ \text { e-ISBN } & 9789048542062 \\ \text { DOI } & 10.5117 / 9789462989504 \\ \text { NUR } & 670\end{array}$

\section{(9) (1) $\Theta \Theta$}

\section{Creative Commons License CC BY NC ND}

(http://creativecommons.org/licenses/by-nc-nd/3.o)

@ All authors / Amsterdam University Press B.V., Amsterdam 2020

Some rights reserved. Without limiting the rights under copyright reserved above, any part of this book may be reproduced, stored in or introduced into a retrieval system, or transmitted, in any form or by any means (electronic, mechanical, photocopying, recording or otherwise).

Every effort has been made to obtain permission to use all copyrighted illustrations reproduced in this book. Nonetheless, whosoever believes to have rights to this material is advised to contact the publisher. 


\section{Table of Contents}

$\begin{array}{lr}\text { List of Figures } & 9\end{array}$

Acknowledgements 11

1. Introduction 13

$\begin{array}{ll}\text { Meet Rika } & 16\end{array}$

Methods 20

Digital Kinship as Intimate Mundane Co-presence 22

Friendly Surveillance and Care at a Distance $\quad 25$

Kinship Across Three Cities, Generations and Cultures $\quad 27$

$\begin{array}{ll}\text { Shanghai } & 28\end{array}$

Tokyo 30

Melbourne 31

Structure of the Book 33

\section{Section I Digital Kinship}

2. Platform Genealogies 43

Japan: LINE: A Post 3/11 Social Media 45

China: WeChat $\quad 48$

Melbourne: Facebook, WhatsApp and Instagram 50

Locating the Platforms Across the Sites: Emojis/Stamps/Stickers 54

3. Friendly Social Surveillance 61

Understanding Contemporary Surveillance: A Familial Model 63

Care at a Distance: Examples of Families and Friendly Surveillance 65

$\begin{array}{ll}\text { Melbourne } & 65\end{array}$

$\begin{array}{ll}\text { Tokyo } & 68\end{array}$

$\begin{array}{ll}\text { Shanghai } & 71\end{array}$

Cultural Understandings of Friendly Surveillance $\quad 73$ 


\section{Section II Playful Kinship}

4. Digital Gifts and Rituals 79

The Cultural Dimensions of Gifts and Rituals 80

$\begin{array}{ll}\text { Digital Gifts and Domestic Care } & 84\end{array}$

$\begin{array}{ll}\text { Digital Gifts as Intimate Co-presence } & 87\end{array}$

Keeping While Giving $\quad 89$

Conclusion: Gifts of Presence/Presents 90

5. Playful Haptics in Families 93

Reading Gestures $\quad 95$

Haptic Play and Screens $\quad 96$

Haptic Rhythms $\quad 99$

Haptic Play Poetry 103

Haptic Play Cadences (Co-present Frequency) 106

$\begin{array}{ll}\text { Conclusion: Playful Encounters } & 109\end{array}$

\section{Section III Visualizing Kinship}

6. Personal Visual Collecting and Self-Cataloguing 113

The Politics of Collecting: Sharing and Non-sharing, Group Archive or Self-catalogue $\quad 115$

$\begin{array}{ll}\text { Tokyo } & 118\end{array}$

Melbourne $\quad 123$

$\begin{array}{ll}\text { Shanghai } & 127\end{array}$

$\begin{array}{ll}\text { Conclusion: Sharing and Non-sharing } & 131\end{array}$

7. Visual Generational Genres 137

Co-present Eating: Sharing Food Moments 139

Co-present Mobility: Sharing Travel Experiences 146

$\begin{array}{ll}\text { Conclusion } & 147\end{array}$

\section{Section IV Co-futuring Kinship}

8. Re-imagining Digital Care and Health 153

Mundane Mobile Games as Quotidian Digital Health $\quad 154$

Applified and Datafied: Quantified Self and Digital Health $\quad 156$

$\begin{array}{lr}\text { Feeling data } & 15^{8}\end{array}$

Conclusion 160 
9. Quotidian Care at a Distance

163

$\begin{array}{ll}\text { Informal Care } & 165\end{array}$

$\begin{array}{ll}\text { Digital Care } & 167\end{array}$

WeChat and Informal Care $\quad 171$

$\begin{array}{ll}\text { Careful Apps in Melbourne } & 178\end{array}$

$\begin{array}{ll}\text { Conclusion } & 183\end{array}$

10. Conclusion 187

$\begin{array}{ll}\text { Continuity and Discontinuity } & 189\end{array}$

Implications for Digital Media Practices in Households $\quad 191$

$\begin{array}{ll}\text { Author Biographies } & 197\end{array}$

$\begin{array}{ll}\text { Index } & 201\end{array}$ 



\section{List of Figures}

$\begin{array}{lll}\text { Figure 1.1 Rika's LINE picture to her mum } & 18\end{array}$

Figure 1.2 Sending messages, stamp and videos to family members on LINE $\quad 20$

Figure 2.1 Chat with rabbit stamp on LINE 47

Figure 2.2 Yolanda's father sharing his daily jogging record in the family WeChat group $\quad 50$

Figure 2.3 Facebook page from our Melbourne participants $\quad 51$

$\begin{array}{lll}\text { Figure 2.4 WhatsApp } & 53\end{array}$

Figure 3.1 Maki and Eriko exchanged messages and photos on LINE for confirming the safety while travelling abroad $\quad 70$

$\begin{array}{lll}\text { Figure 4.1 LINE stamps for mums } & 80\end{array}$

Figure 4.2 WeChat Happy New Year gifting 84

Figure 4.3 Saori and symbolic gift giving through maintenance on LINE 86

$\begin{array}{lll}\text { Figure 4.4 Kevin's family WeChat group } & 88\end{array}$

Figure 4.5 Satomi and co-presence as gift 90

Figure 5.1 Susan using her iPad pointer (so her fingers don't touch the screen) 94

Figure 5.2 Yuto's journey via Swarm mapping 97

Figure 5.3 Yuto's smartphone is often in his pocket. He took it out during the interview 100

$\begin{array}{lll}\text { Figure 5.4 LINE PokoPoko } & 102\end{array}$

Figure 5.5 Takeshi's right thumb became bigger than his left thumb 103

Figure 5.6 A mother's poem shared by her daughter on Weibo.com 104

$\begin{array}{lll}\text { Figure 5.7 WeChat emoticons } & 105\end{array}$

Figure 5.8 An example of WeChat-provided stickers 105

Figure 5.9 Screenshot of Taylor's family WeChat group 106

Figure 5.10 Screenshot of Tiffany's family WeChat group 107

$\begin{array}{lll}\text { Figure 6.1 } & \text { Mari's Instagram images } & 120\end{array}$

Figure 6.2 Nozomi's Lunch photographed and shared 121

Figure 6.3 Tiffany sharing a photo of her school dumplings with her family $\quad 128$

Figure 6.4 Posts by Ben 129

$\begin{array}{lll}\text { Figure 7.1 Rika's travel images } & 140\end{array}$

Figure 7.2 WeChat moments, sharing food in Shanghai 144

$\begin{array}{lll}\text { Figure 7.3 Chun's shared pictures } & 145\end{array}$ 
Figure 7.4 Jia's images on holiday

146

Figure 9.1 A moving echo image of the embryo provided by the gynecologist and shared with Serika's family members on Mitene

Figure 9.2 Screenshot of a meditation app, which gives instructions according to the user's heart rate. The text translates to "training to look outside with concentration"

Figure 9.3 Masami is playing game, reversi, on tablet PC 170

Figure 9.4 Hiroshi likes playing "old" game on desktop computer $\quad{ }_{171}$

Figure 9.5 Screenshot of Li Wei's family group chat 177

$\begin{array}{lll}\text { Figure 10.1 Rika's Instagram } & 188\end{array}$ 


\section{Acknowledgements}

The authors would like to thank all the participants in Melbourne, Tokyo and Shanghai for allowing us into their homes and lives. Thank you for sharing your insights and stories.

This book is the result of an Australian Research Council (ARC) Linkage project Locating the Mobile with Intel, Keio University and Fudan University. We would like to thank Distinguished Professor Genevieve Bell for her early insights and contributions. We also acknowledge the great editorial work by Klare Lanson and would like to thank Shihui Gui and Qianqian You for their research assistance. Larissa would like to also thank RMIT University and her Design \& Creative Practice Platform colleagues.

And, of course, the authors wish to thank friends, family and colleaguestheir support cannot be underestimated. 



\title{
1. Introduction
}

\begin{abstract}
In this introductory chapter we begin with one of our participants, Rika, as she uses her smartphone practices to help create a sense of care at a distance with her aging mother-what we call Digital Kinship. We then turn to contextualizing the methods deployed over the three years in three locations and how each of the three cultural contexts informs different rituals around data use. We discuss how Digital Kinship can make sense of the paradoxical role of surveillance in an age of datafication through "friendly surveillance" and "care at a distance."
\end{abstract}

Keywords: data; dataveillance; care at a distance; intergenerational; Digital Kinship; ethnography

Data — and its locative possibilities and potentialities — can be found in almost all our quotidian moments. Waiting at the bus stop. Riding home on a bike. Searching for the nearest bookshop. Grabbing a bite to eat on the run. Moving from one work meeting to the next. A school pickup. A Sunday brunch with family. In these everyday moments, the mobile phone is on hand. Listening. Tracking. Connecting.

For many, the datafication of everyday life has both invisible and visible dimensions and implications through social mobile media. Datafication - the "conversion of qualitative aspects of life into quantified data" (Ruckenstein and Schüll 2017, 261) —unevenly occupies many of our lives in ways we are yet to fully understand. Sometimes the narratives between practice and perception converge, other times they diverge. Datafication occupies a paradoxical role in our lives-empowering and yet exploiting, visualizing while camouflaging, user-generated and yet platform (corporate) personalized.

Mobile media devices such as smartphones and Apple Watches_-along with apps like Strava and Instagram - weave multiple data trails of intentional and unintentional tracking. From self to social to corporate, the data

Hjorth, L., K. Ohashi, J. Sinanan, H. Horst, S. Pink, F. Kato, B. Zhou, Digital Media Practices in Households: Kinship through Data. Amsterdam: Amsterdam University Press, 2020 DOI 10.5117/9789462989504_CHO1 
trails and tracks are as thick as they are dynamic. Examples are endless. Sometimes we consciously check on a relative through social media, other times we unthinkingly watch ourselves and loved ones on a post. Sometimes we reflect on a deceased loved one with someone in another country via a post. Sometimes our data trails take a life of their own, in ways we are still yet to fully understand.

Much scholarship has been conducted into these two camps of beliefone, the dataveillance (Ruppert 2011; van Dijck 2014; Lupton 2016) group who see empowerment narratives underscored by obligations often not understood by users; the other group more aligned with Quantified Self (QS) ideals that see the body as the laboratory for creative reflection and self-knowledge through numbers. Indeed, much is still to be learnt from ethnographic and creative explorations into everyday datafication as a way to reimagine ourselves within social dynamics (Sharon 2017; Pink and Fors 2017; Khot et al. 2016; Fors et al. 2019). Such research into self-tracking and wearables has demonstrated how personal data can become part of people's personal and collective digital lives - whether as part of ordinary everyday life, or within the context of the QS movement (e.g. Lupton 2016). However, there is another dimension to mobile tracking that is less immediately visible, yet perhaps even more pervasive than digital self-tracking.

In the literature, the role of data to care at a distance - especially between the generations - has been relatively overlooked. For instance, how can we learn from quotidian intergenerational practices to reflect upon the changing role of care with datafication? What are some of the making-do practices emerging around intergenerational care at a distance?

In Digital Media Practices in Households focus on intergenerational mobile media practices, through an analysis of how these are lived and experienced across three different social and cultural contexts. These practices are played out in an ambivalent and paradoxical space that is at the intersection of intimacy, care and data transition. Attention to such practices account for, but goes beyond, the emphasis on personal data, spans different types of platform and media practices, and brings attention to the intergenerational and cross-cultural understandings that are often left out of the debate. We seek to understand datafication in terms of the often-invisible care work done in intergenerational relationships. We bring care and media practice together to think about contemporary forms of kinship that marry the digital, social and material in complex ways.

In Digital Media Practices in Households we trace the cross-cultural and intergenerational role of mobile media practices in three locations - Shanghai, Tokyo and Melbourne. Through the concept of Digital Kinship, we bring 
together the continuities and discontinuities in and around the negotiation of mundane intimacies in the digital and non-digital worlds. In this book we seek to connect the discontinuities with the continuities through four key kinship concepts—Digital, Playful, Visualizing, Co-futuring — and show how these elements of kinship are played out through culturally specific modes of the intimate and mundane.

From social media like LINE, WhatsApp and WeChat and self-tracking health apps on smartphones to wearables like Apple watches, this book explores the multiple ways in which intergenerational practices play out around mobile media for care at a distance. This can involve locative and non-locative possibilities. We recognize that quotidian forms of locative media are often embedded in social and mobile media practices. As Rowan Wilken notes in The Cultural Economies of Locative Media (2019), within the all-pervasiveness of everyday mobile media, we can find multiple and contesting forms, textures, and gradations of location that inform our contemporary ways of being in place $(2019,5)$. Thus, understanding locative media needs to be done in the context of the embedded mobile media practices.

Entwined within our exploration of mobile media in households and familial contexts is the integral role of care within contemporary media practices. Care, as we argue, isn't just a practice for feminist or social services but rather crucial to an ethics of media practice (Mol 2009; Bellacasa 2018). As the fallout from the Cambridge Analytica debacle still resonates around notions of trust, bringing care (ethically, theoretically, conceptually and methodologically) to media practices is key (Gold and Klein 2019). We redeploy Jeanette Pols' notion of care at a distance (2012) — originally used in telecare settings to explore the role of technology to enhance relationships when used in unison, not replacing, people - to consider the tacit, informal and mundane ways in which mobile media does often invisible care work in everyday intimate relations.

Given the above ambitions, Digital Media Practices in Households is not a conventional academic book. In it we seek to bring readers into the mobile and digital family lives and everyday worlds of participants, across three different cultural and national contexts. This means leading our discussion by example, rather than by theory. In particular, through the practices of our participants we reflect on the quotidian and often-invisible forms of care at a distance constitute contemporary Digital Kinship.

Through cross-cultural examples, we seek to explore the ways in which place and context inform particular rituals of belonging. We believe this helps to bring to the fore the socially active micro-moments that matter to our participants, and our analysis. In doing so, we invite readers into 
an interpretive process that is based on ethnographic encounters with participants in the places where mobile media practices are meaningful to them. Through this process, we seek to explore the everyday intimate and mundane meanings of caring and relationality that, in turn, has methodological consequences and makes a substantive contribution to the study of intergenerational mobile media practices across cultures.

In this introductory chapter we begin with one of our participants, Rika. We visit her experiences in negotiating her aging mother's independence and how her smartphone practices help to create a sense of care at a distance - what we call Digital Kinship. We then turn to contextualizing the methods deployed over the three years in three locations. Then we discuss understanding Digital Kinship as a form of intimate mundane co-presence. This is followed by a reflection upon the ways in which we seek to understand the paradoxical role of surveillance in an age of datafication, through "friendly surveillance" and "care at a distance." Having outlined key concepts, we then contextualize our three contrasting sites that can provide insight into some of the salient and culturally specific notions. We reflect upon how understanding data and care through an intergenerational lens can provide nuanced insight. We then discuss the book structure. Now let us turn to Rika.

\section{Meet Rika}

In 2014 we met 32-year-old female flight attendant Rika who lived in a bedsit about an hour by train from Tokyo. Just a stone's throw away from her apartment, Rika's only family member-her 72-year-old mother-lived alone. Rika would often leave home for work early in the morning and return home late. Rika and her mother respected each other's daily rhythms by living separately. While traditionally families in Japan would live together in bygone times, now it is common for them to live apart. However, Rika's smartphone (sumaho) provided a care at a distance whereby Rika was able to monitor her elderly mother through the constant co-presence of social mobile media.

Like many Japanese of her generation, Rika grew up with the mobile (keitai) and viewed her phone as an integral part of her everyday life. Rika's first keitai was the one that her mother bought her when she was in junior high school, with the intention of using the phone as a form of personal security — or what Misa Matsuda (2009) calls "mom in the pocket" - to accompany her constantly when travelling from after-school cram (or "juku") 
school. Since her first keitai, Rika and her mother had a long history communicating phatic, logistic, and emotional messages through and by the mobile phone. As the mobile phone had grown and transformed into the sumaho, so too had Rika and her mother's relationship evolved through its constant co-presence, so much so that the mobile played an integral role in maintaining their broader kinship network.

After graduating from university, the mobile phone became even more important in maintaining Rika's relationship with her mother, as Rika started to fly around the world as a flight attendant. Overseas flights occasionally caused unexpected delays. As Rika described, “I couldn't come back to Japan when there was a flood in Thailand and a volcanic explosion in Iceland. In these situations, if it wasn't for mobile phones, my mother would not have been able to find out if I was OK." Her airline policy stated that family members of employees were not allowed to make calls directly to the airline if a hijacking occurred. This made the mobile phone indispensable in allowing Rika to communicate with her mother especially when she was abroad.

Rika often did a "check-in" on Facebook when she went abroad on business. One reason was to let her mother know where she was. Her mother did not have her own Facebook account. So, Rika kept hers logged-on via the tablet PC and gave it to her mother, who could then check Rika's timeline without it being bothersome. Previously, she used to give a paper-based hotel list to her mother, but with Facebook "check-in" functions this was no longer needed. Social media platform LINE also played a key role in connecting the pair when they were away from each other. They could talk or chat for free by using LINE — the most used social media app in Japan — when they have WiFi connection, regardless of their location.

In this opening vignette we see Rika utilizing mobile media to share both co-presence and co-location with her mother as a type of care at a distance. Over the three years of the fieldwork, Rika went from using locative media as a tool for care at a distance with her mum to becoming a mum herself. Through these transitions, Rika adapted media practices in accordance with the rhythm of her everyday life. We understand how Japanese social media platforms LINE and Facebook were used for digital and visual reflection of - and for-kinship. These digital kinship practices played a key role in the maintenance of social rituals and gift giving economies as well as affording new ways to express mundane intimacies in playful and visual modes. Through the study of Rika's media practices over three years, we recognized that the relationship between mother and daughter was gradually changing as they began to adopt new mobile media technologies. 


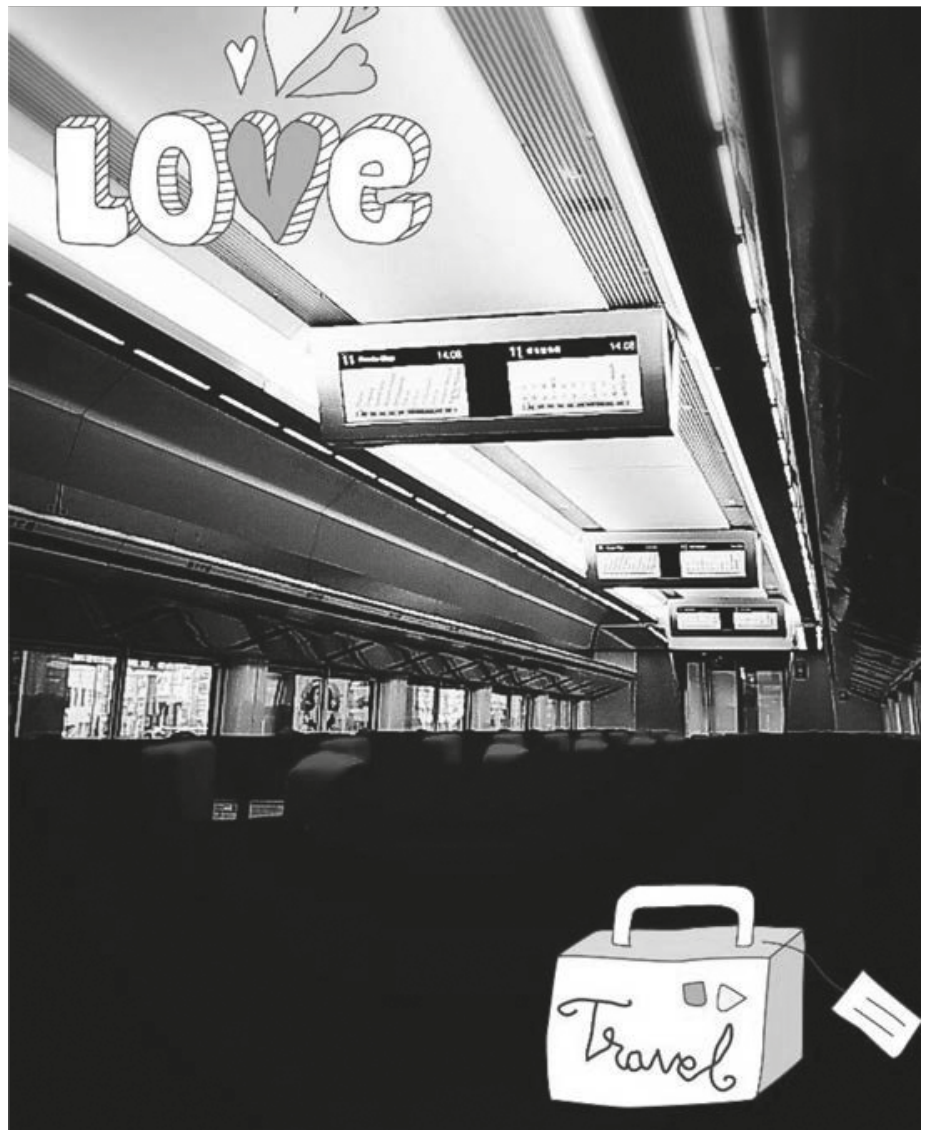

Figure 1.1: Rika's LINE picture to her mum

In Rika's exchange with her mother we can understand the power of digital media to create different forms of care at a distance. For many feminist scholars, care cultures are an important site for affective, emotional and unpaid labor (Mol 2009). Fields such as nursing and teaching are often underpaid despite the pivotal role played in maintaining many societies. The role of care as a feminized form of labor often plays out in many work and social contexts with particular "feeling rules" (Hochschild 1979) being expected. The maintenance of particular feeling rules often involves a type of informal surveillance.

Care has always had a complex relationship to surveillance (Bellacasa 2017), but digital media obscures this imbrication further. Mobile technologies have been deployed as ambient forms of surveillance between family members as evidenced through the substantial research of Misa Matsuda (2009) in Japan. More recently, work has begun to emerge around 
mundane, emergent practices that maintain intimacy in families (Clark 2012; Sengupta 2012; Leaver 2017; Burrows 2017), school surveillance (Shade and Singh 2016) and intergenerational "friendly surveillance" (Hjorth et al. 2017). We know very little about the ways mobile media practices relating to care and intimacy_-what Tama Leaver calls "intimate surveillance" (2017) - are being played out in everyday familial contexts. And how, in turn, these mobile intimacy practices are recalibrating how surveillance is being conceptualized.

In Rika's mundane and intimate exchanges with her mother through social mobile media we see the persistence of previous media rituals-most notably the keitai. We begin to comprehend how the keitai cultures have become part of the fabric of ritualization that represents an extension of existing types of care and gift giving practices as well as new ways to be co-present - a mode of electronic proximity that expands upon temporal, spatial and geographic distances. These modes of co-presence afford ways in which the mobile phone can operate materially and symbolically, expanding upon existing care cultures through the ambience of co-presence. A message or "stamps" (stickers) on LINE can send feelings of care and responsibility-reminding us of the ongoing role of the mobile phone in gift giving practices (Taylor and Harper 2002). As we will see in Chapter 2, the uptake of different platforms in the specific contexts highlight that cultures enhance particular modes of "platformativity" (Lamarre 2017) as much as platforms frame our ways of seeing. In Japan, LINE dominates, while in China WeChat is all-pervasive. In Australia there is a mixture of Facebook, Instagram and WhatsApp.

Paralinguistics - like emojis, stamps and stickers—provide emotional and facial clues for mediated environments. Each platform has its own customized from of emojis, further enhancing particular cultural nuances and norms. They also expand upon discourses around mobile parenting research by affording unilateral social or friendly surveillance between children, their parents and grandparents. During the age of the keitai, children were the ones monitored by their parents. Now, however, in the age of the sumaho, it is the grown children who are monitoring their elderly parents in what can be understood as a "social" (Marwick 2012), friendly or careful surveillance (Hjorth, Richardson and Balmford 2016).

Underlining the tension between enduring and changing rituals of kinship, Rika's story and use of digital forms of co-presence also demonstrate how new forms of kinship are being interwoven within the everyday. Understanding Digital Kinship is central to this book. Through the role of locative media use and non-use, we explore how cultural and generational 


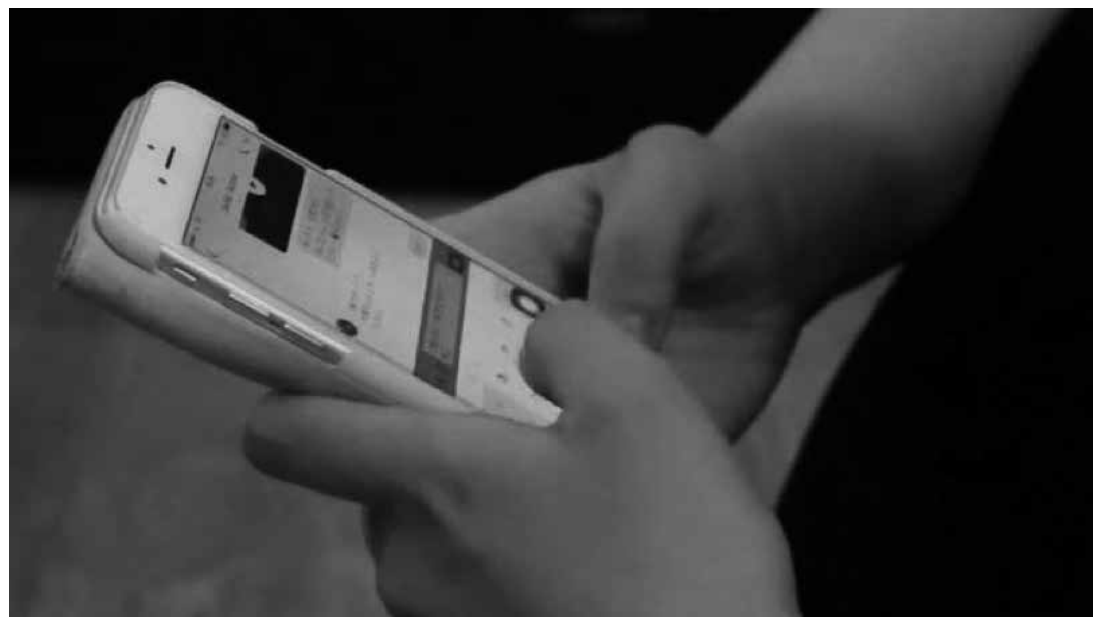

Figure 1.2: Sending messages, stamps and videos to family members on LINE

differences are informing practices of care at a distance, social surveillance and maintaining intimacy. The significance of "non-use" is now a significant part of the repertoire of everyday media practices (Baumer, Ames, Burrell, Brubaker and Dourish 2015; Baumer, Adams and Khovanskaya 2013; Satchell and Dourish 2009) and researchers are starting to realize the importance of understanding media practices as part of a continuum that involves use and non-use in relation to the rhythms and activities of everyday life.

In the remainder of this introductory chapter we outline some of the key concepts and methods included in this book. First, we begin with a brief methods section. We then discuss the role of Digital Kinship as intimate mundane co-presence. This is followed by a review into some of the ways in which surveillance has been theorized and consider how it is being recalibrated through familial locative media use. Then we examine our fieldsite locations, ethnographic methods, and how ethnography can provide valuable insight into understanding locative media in practice. We conclude with a discussion of the book's structure and chapter outlines.

\section{Methods}

Mobile and haptic media play an increasingly central role in intergenerational and transnational relationships and intimacies. To understand how locative, social and mobile media fits into the rhythms of everyday life - with its mundane routines and intimacies - requires us to go beyond standard interviewing methods. Instead we developed ethnographic techniques that 
enable the researcher to engage empathetically with people's intimate experiences in mundane life (Pink, Ardèvol and Lanzeni 2016). Often mundane experience of digital technologies is difficult to access or to observe as a researcher, precisely because it happens at moments when people are alone and in situations where they are unlikely to usually share with others, let alone with researchers.

We employed mobile media as a tool for the researchers and participants, and also positioned mobile devices and software as tools in themselves. This approach was predicated on the idea that mobile media such as smartphones and apps work as a very intimate and mundane objects, allowing for ethnographic research to be undertaken without participants feeling the need to be 'on display' or perform activities that were not part of their usual lifeworlds.

Given that locative media is often used on the move, this makes it even harder to trace. Earlier in the research we had considered using Go-Pro video cameras and giving them to participants, however we felt such a technology would be too foreign and thus destabilize everyday familiar practices. Much of locative media that is activated intentionally, occurs while moving and waiting to move - at a bus stop, planning a driving trip, on public transport, just before getting up in the morning or just about to go to sleep at night, tagging locations while on holiday, or while taking a moment out of a social situation. That is, transitional moments.

To conduct an ethnography of Digital Kinship through locative media means developing techniques to understand these practices as situated within the familial rhythms of everyday life. This means understanding it as part of social mobile media practices more generally. In particular, our study followed 12 households over three years (2014-2017) within the three very different locations we strategically selected (Melbourne, Tokyo and Shanghai) to gain a sense of cultural differences and similarities with respect to intergenerational use. In each cultural context, one local informant was the key researcher to ensure for nuanced understandings of the linguistic, social and cultural practices. In each location we had one Chief Investigator (CI) who worked with a research assistant to recruit a diverse cohort of families in different parts of the three cities. Given the attrition rate over three years, we began with 36 households and ended with 30 households.

In Tokyo and Shanghai, interviews were held in Japanese and Chinese, then transcribed into English. Interviews were predominantly held in the home except when participants, for convenience, requested alternative sites. In this three-year study we were keen to put locative media into context-culturally, socially, linguistically and technologically. This required 
that we deploy methods that sought to understand locative media as part of the assemblage of social mobile media practices. We used video interviews focused on the participants' discussion of their everyday practices through their hand gestures in and around the device. This technique-tactile ethnography - allowed us to explore some of the tacit feelings and practices informing the rituals. There is a growing need to understand screens as part of a haptic ecology that moves in, across and around the screen-which mobile media can help elicit (Pink, Sinanan, Hjorth and Horst 2016; Richardson and Hjorth 2018). For example, often in re-enactment, the hands would role play the movements across the screen in ways that sometimes provided more insight than what was being articulated by the participant. This would then allow us to ask more questions about the gestures as a way to discuss the performative and tacit forms of practice and proprioception. We need to consider what researching through and by the hands might add to our methods and theorization for mobile media and screen research. These haptic practices inform how we interact, experience and understand locative media in our everyday.

We asked participants to show us a few activities - scenarios of use, 'one day in the life of' (re-enacting a typical day of mobile media use, usually yesterday), app walkthroughs - while they talked us through the images, texts and apps they use and why. This approach to ethnography allows us to understand motivations for use which can put digital media into practice: that is, what certain images, words and rituals mean as part of broader kinship practices. The project sought to put into context these rituals rather than seeing the digital as a mere 'disruption.' Just as no method is left untouched by the field (Lury and Wakeford 2011), so too does the relationship between the digital and kinship become one about continuity across articulated and tacit lifeworlds and experiences. These methods allowed us to understand the dynamic relationship between the interfaces, bodies and cultures framing particular forms of mundane Digital Kingship.

\section{Digital Kinship as Intimate Mundane Co-presence}

Understanding Digital Kinship has been central to the broader research project. Over three years (2014-2018) our work traced the role of cross-cultural and intergenerational practices in three locations-Shanghai, Tokyo and Melbourne. These contrasting urban contexts provided different examples of the "digital city" as a complex cartography that involves contested interfaces, vulnerable agencies and placemaking (Foth, Brynskov and Ojala 2015). 
While urban studies scholars have studied the role of smartphones in public engagement and citizenship, it is the more informal practices within the home-and how the home is carried with us through the phone (Morley 2003) across different spaces, places and cultures - that is central to our notion of Digital Kinship.

Through the notion of Digital Kinship, we bring together the continuities and discontinuities in and around digital media and intimacy. Digital Kinship embeds the study into broader debates about the changing (or residual) nature of what it means to be a "family" in an age of networked media as argued by Clark (2012) as well as Horst and Miller (2012). Kinship has always been important to ethnographic understandings of culture. With the added dimension of the digital, we see how kinship moves in and out of online and offline spaces and, in turn, how these spaces have come to develop their own histories, connections and memories.

Doing intimacy within contemporary contexts requires acknowledging the ways in which it can be public and community orientated (Jamieson 2011) - especially through the gradations of publics afforded by social media. In The Feeling of Kinship, David Eng (2010) puts forth a theory of intersectionality whereby previously conceived private and individual psychic structures are transformed into collective ones- that is, the feeling of kinship. Kinship, or in this case Digital Kinship, is central to understanding how contemporary locative media uses and non-uses reflect doing intimacy and boundary work.

As Lynn Jamieson (2011) has noted in her detailed historicization of intimacy, the dichotomy between "private intimacy" and "public community" is deeply flawed. Drawing from feminist literature, Jamieson argues that much of contemporary "doing intimacy" involves community and civic engagement - a process that sees a complete transformation of the role of intimacy in and around the notion of family. Research on couples offer a "mixed picture" on the place of boundary work in doing intimacy. In the work of Lasén and Casado (2012) they discuss how heterosexual couples deploy mobile media in ways to express different forms of intimacy and boundary work - processes that can involve "quiets" and "disquiets."

Understanding digital kinship requires us to think about intimate copresence and the mundane. Co-presence has been an important term in new media, internet and mobile communication research. Outlined by Erving Goffman (1959) as an integral part of everyday presentations of self, the notion of co-presence sees all forms of intimacy as mediated. The work of Mimi Ito and Daisuke Okabe (2005) was seminal in adapting this concept to mobile phone contexts in Japan. Moreover, debates around the 
co-present role of the researcher in, and around, the online (and its relation to co-location) have taken on further significance with ethnographies arguing that the field is always mediated (Beaulieu 2010), if not by technology then by language, gestures, subjectivities and memories. For Anne Beaulieu, this requires ethnographers to think not only of the politics and practice of co-presence but also co-location.

The intimate mundane brings together two strands in recent literatures that are concerned with the embodied and affective dimensions of mundane everyday life at home and with digital media: the role of the mundane and banal as a site for analyzing power relations and rituals; and the role of the intimate as entanglement within everyday digital media practices. Intimacy here must be understood beyond western or Anglophonic traditional notions which privilege the face-to-face as wrongly unmediated (Jamieson 2011). Rather, intimacy needs to be understood on various levels - between individuals, groups and societies — while also acknowledging the role of such concepts as Herzfeld's "cultural intimacy."

For Herzfeld, cultural contexts inform the ways in which intimacy plays out in everyday practices. Herzfeld $(1997,44)$ notes that the "intimate seeps into the public spheres that have themselves been magnified by the technologies of mass mediation." Here cultural intimacy takes three forms: historical, institutional, and geographical. Through a notion of cultural intimacy, we can reconfigure intimate publics as they move in and out of the digital practices.

As Laurent Berlant $(1999,281)$ observed in the last century, intimacy had taken on new geographies and forms of mobility, most notably as a kind of "publicness." In keeping with Michael Warner (2002), Berlant sought to queer traditional and static notions of the public and citizenship by addressing the often tacit and yet increasingly important role of the intimate. In a digital material environment, intimate relations are not simply performed in pairs, bounded groups or cultural contexts; they also traverse the online and offline. This traversing sees physically public worlds entangled by electronic privacy, and an electronic public that is geographically private. As Mimi Sheller asserts "there are new modes of public-in-private and private-in-public that disrupt commonly held spatial models of these as two separate 'spheres'” (2004, 39).

As noted by others (Dobson 2015; Berlant 1999; Warner 2002; Hjorth and Arnold 2013), the idea that forms of intimacy might be generated in contexts that are at the same time public is not new. Mobile media amplify inner subjectivities as they conform to existing socio-cultural rituals and practices. As one of the most intimate devices in everyday life, mobile phones are 
vehicles for haunting upon multiple material, symbolic, and immaterial dimensions. They are vessels for and of our intimacies and emotions, shaping and being shaped by affective bonds. Hjorth and Arnold (2013) have argued that mobile media are also increasingly vessels for intimate publics and mobile intimacy. Here we can understand mobile intimacy as an overlay woven between the electronic with the social, and the emotional with co-present. Intimacy is a multilayered and contextual concept.

Advancing this further, they have proposed a recalibration of intimate publics in an age of social media, through which we can understand the competing histories, identities and practices within the Asia-Pacific region. This work builds on the research on intimacy and mobile telephony (Hjorth 2007; Prøitz 2007) that has shown how technology invites new forms of presence and proximity. The bodily presence and emotions are not lesser, but different from the traditional. For Marika Lüders (2008), what was previously considered private is no longer restricted to the private sphere. This relationship between the private and intimacy is even more complicated by social media (Hjorth 2009). Locative media, coupled with algorithms and Big Data, have challenged how media, control and intimacy are conceptualized and practiced (Andrejevic 2006; Andrejevic, Hearn and Kennedy 2015). And, in turn, how care and intimacy is playing out through the media practices.

\section{Friendly Surveillance and Care at a Distance}

While locative media is becoming a default in many mobile apps, their usage and non-usage speak to ways in which people curate their intimate and yet public lives. For some, the intimate and public are interwoven. For others, they use media to re-create boundaries between the intimate and private in a world in which these concepts seem to blur. Locative media has provided much food for thought in rethinking privacy (Gazzard 2011; Farman 2011; de Souza e Silva and Frith 2012). Here privacy isn't seen as something we possess (or don't) but rather something we constantly do and define through practice (Dourish and Anderson 2006).

Although there has been discussion about corporate and governmental surveillance in an age of Big Data (e.g. Farman 2010; Andrejevic 2006; Cincotta, Ashford, and Michael 2011), the rise of new forms of social surveillance in families (Marwick 2012) is creating an additional — and to date underresearched-layer of everyday practices layered by locative media (Clark 2012; Sengupta 2012). We know very little about the ways locative media practices relating to privacy, intimacy and surveillance are being played 
out in everyday family contexts, in what way these impact on how, when and where locative media are used, or the implications of these practices for how place and time are experienced. Through studying the messiness of practice across generations and cultures we can begin to think through complex ways in which locative media is used to maintain intimate relations, especially at a distance. To understand locative media in practice requires new approaches to surveillance and its relationship to care and intimacy. In this book, we situate locative media as part of broader social mobile media practices.

Lee Humphreys (2013) identifies three kinds of surveillance involved within social media practice. In addition to the traditional notion of surveillance - characterized by its non-transparency by an authority (i.e. government or corporation) — Humphreys identifies three other kinds of surveillance: voluntary panopticon, lateral surveillance, and self-surveillance. There are other types of surveillance emerging-lateral and social. Lateral surveillance is the asymmetrical, nontransparent monitoring of citizens by one another (Andrejevic 2006).

We saw this practice in our fieldwork, epitomized by 30-year-old Melbourne participant Catherine and the watching of her girlfriend (30-year-old Susan) in the Uber through the app's tracking functions. There are many apps that allow citizens to monitor other citizens' behavior through nonreciprocal forms of watching. Every day people can search for information about other citizens without their knowledge or permission.

The advent of various social mobile media platforms has given rise to other forms of lateral surveillance such as "social surveillance" (Marwick 2012), which suggests a mutual surveillance among actors using social media. Like lateral surveillance, social surveillance involves nonhierarchical forms of monitoring (i.e. not involving the state or corporate entities) among everyday people. Unlike lateral surveillance, social surveillance suggests that people engage in permissible and reciprocal forms of watching. We will discuss this in more detail in Chapter 3 on "friendly surveillance."

As we have seen in fieldwork, tinkering and perpetual modulation are two key practices we identify in participant's practices. Over time, the relationship between intimates and media evolve and dissipate while forming a rhythm of careful maintenance and modifying expectations. Throughout this book you will meet our participants and see a variety of ways in which different relationships and types of kinship are played out and through locative media use. These practices take on various forms of care, co-presence and intimacy and are best understood through ethnography. In order to locate the study, we will briefly outline the three locations, which we have 
chosen for their diversity in adoption of locative media over time. All three locations have very different mobile phone cultures and telecommunication regulatory structures.

For Pols, care at a distance is the processes whereby digital media ambiently reinforces, rather than replaces, face-to-face contact. In her study of digital technologies in palliative care settings, Pols notes that media can be useful only when used in unison (2012). As we will show in this book, intergenerational usage of mobile technologies showed the informal ways grandparents, parents and children were using the technologies to provide a sense of continuity - what we call Digital Kinship. Parents have always found ways to spy on their children, and vice versa. However how this watching takes on the overlays of the mobile, social and locative media creates new types of "sense-making."

\section{Kinship Across Three Cities, Generations and Cultures}

The research discussed in this book was developed across three cities in the Asia-Pacific region: Melbourne (Australia), Shanghai (China) and Tokyo (Japan). Tokyo, Shanghai and Melbourne provide compelling stages in locative media practices. Tokyo offers one of the longest examples of everyday mobile phone use. Shanghai represents a location with a rapid and large-scale uptake of locative media by predominantly generation $\mathrm{Y}$, the $b a$ ling hou (CNNIC 2011; Hjorth and Pink 2014) and generation Z, and also the "millenials." Melbourne offers an example of fast locative media adoption through high percentages of smartphone penetration (Australian Bureau of Statistics 2011; Our Mobile Planet 2012). By contrasting and comparing these three divergent cultures through locative media use and non-use we can gain deeper insights into mobile media as part of emerging twenty-first century everyday cultural practices.

The rise of the smartphones within urban contexts has generated much interdisciplinary debate from urban studies and human computer interaction (HCI) to name a few. Smartphones, as part of the Internet of Things (IoT), have been discussed as both a vehicle for empowerment (open data) and exploitation (surveillance). As cities such as Tokyo embrace smart city infrastructure that allows for data collection, analysis and evidence-based policy interventions around environmental responsiveness and sustainability (Hobson and Marvin 2017), the role of these digital technologies for social innovation (especially informal wellbeing and social inclusion for older adults) becomes increasingly important. And yet, understanding the 
informal practices of kinship care which often occurs in the home-especially in terms of intergenerational care of older adults—has been left out of the discussion (Hasan and Linger 2016; Koch 2010; Muramatsu and Yarime 2011). As we explore in the last section of this book, understanding Digital Kinship can provide insight for future planning around super-aging contexts.

Although historically kinship and the structures of family life vary significantly across the three locations (e.g. Daniels 2010; Fong 2004; Kitaoji 1971; Xu and Xia 2014; Wallis 2013; Nonoyama 2009; Amagasa 2012; Matsuda 2009; Dobashi 2006; Hjorth and Arnold 2013), one of the commonalities across all three sites is the changing role of the family with urban migration and growth (Baldassar 2007), which in turn, is changing the ways in which "family" is defined and practiced across all three sites. In particular, an increasing trend towards nuclear families at the same time as a marked growth in the aging population in China and Japan - coupled with policy frameworks-have shaped the structures of families. The concept also acknowledges changing definitions of family in multicultural urban areas such as Melbourne, including the growth in gay, lesbian, bisexual and transgender (GLBT) families, single parents, and families without children. These new forms of families are, in turn, shaping emergent meanings and forms of kinship and relatedness.

In the following section, we briefly outline the three locations to provide readers with a context.

\section{Shanghai}

Over the last 30 years Shanghai has grown into a mega-city with a population of 24.2 million people in 2015. One of the first areas to explore the open-door policy, it is now the largest city in China which is known for the increase in sectors ranging from finance and retail, to real estate and growth in the tertiary sector. Acknowledged for overcrowding and extensive pollution associated with industry and transport, housing has expanded across the region with the increased suburbanization and peri-urbanization of the city adding to the burden of daily commutes. "Temporary" migration has also added to the population and infrastructure burden of the city. Nevertheless, Shanghai attracts such temporary migrants from rural and peri-urban areas of China precisely because of the higher quality of life.

An increase in consumption both of consumer goods, food as well as energy and other resources is prevalent, and scholars have noted the rise of a middle, consumer class (Lewis, Martin and Sun 2016). The growth of the middle class has concurrently increased healthcare and other quality 
of life measures, including lifespan. As Gou observes, another particularly important characteristic of Shanghai is the large proportion of the population over 60 years of age, "22.5 per cent of the overall population in 2009, a figure that is almost double the national average" (2011, n.p.). As a result, the need for communication across generations who might not live in the same region of the city, or the country, makes the understanding of the micro-dynamics of kinship through digital media of central importance.

Like Japan, social media is synonymous with mobile media for most of the older generation. For many millions of people living in regional areas, the use of QQ provided an early entrance into both mobile media and the internet (Hjorth and Arnold 2013). QQ was initially used by young adults who moved away from home as part of a broader trend of geographic and economic mobility unimagined by their parents. By 2010, it was RenRen (meaning people people) - which had the same functionality and design as Facebook - that started to be popular. This was then superseded by the highly popular and easy-to-use stand-alone apps WeChat and Weibo.

Throughout these transitions, young adult children taught their older parents how to use each new platform. Increasingly Chinese families started to form collaborative WeChat groups in order to experience instant online communication no matter how far they lived or worked from each other geographically. This popular new media channel, with its wide range of user adoption and distance-sensitive cases involved in such new family communication patterns, enabled a new way of intergenerational communication between parents and adolescent children. These shifting intergenerational relations need to be reflected in the light of the One-Child policy carried out by Chinese government from the 1980s, which has radically changed the size and intimacy of millions of families in China.

Therefore, the Digital Media Practices in Households research team in Shanghai carried out qualitative research to explore the general questions about how social mobile media use interact with intergenerational relationship within families, using in-depth interviews and scenarios of use (re-enactment) as the main method. A total of 11 family sample groups were collected based on various factors including gender, age, and distance between parents and children. Given the expanding tertiary sector, all of the families in the study included undergraduate students who lived in Shanghai. Parents and children were interviewed separately, consolidating dyadic data of each family from both the elder and the younger generation perspectives. This chosen interview style contributed to avoiding biases in conclusion caused due to partial information collection. Textual analysis on the WeChat interaction screenshots were also carried out. These rich 
first-hand materials made it possible to dig deeper into group online information sharing practices within members of tertiary/university students' families via WeChat.

\section{Tokyo}

In urban Japan the nuclear family consisting of "a couple and child(ren)" has come to be regarded as an exemplary model of the family (Nonoyama 2009). In practice, the composition of the typical family has, in fact, diversified due to the decrease in the number of people getting married, a trend towards marrying later in life, and a decline in the number of children couples and families have. For example, the number of couples married in 2012 was 670 , 000, which was $60 \%$ or 430, ooo less than the level in 1972 (1.1 million).

The average age of first marriage in Japan was 30.8 years old (male), and 29.2 years old (female) in 2012, which signals a rise in the average age of first marriage by 3.0 years (male) and 4.0 years (female) over the last three decades (Ministry of Health, Labor and Welfare 2013). The total fertility rate (TFR) fell to record-low of 1.26 million in 2005 as compared to 4.3 million during the first baby boom from 1947-1949. The number still remains at a low level when compared with countries in Europe and the United States, even though there is a slowly increasing trend in 2011, when the number was 1.39 million (Cabinet Office 2013).

Trends in percent distribution of households by structure of household show that the percentage of one-person households and households of couples has only been increasing in recent years (Statistics and Information Department, Ministry of Health, Labor and Welfare 2012). As of 2010, 25.5\% of every household was a one-person household and $22.6 \%$ of households consist of a couple without children. These statistics illustrate the extent of structural changes to the family in Japan over recent years. In light of this phenomenon, Hisaya Nonoyama (2009), an advocate of the "family lifestyle approach", argues that today, the notion of "family" indicates various forms of family selected by individuals' lifestyles, and family has become an important object of choice for individuals. Thus, within the context of "family" - which is selected and formed as a lifestyle-individuals have to exert active effort to build and maintain "familiar relationships," otherwise $\mathrm{s} /$ he cannot enter, or $\mathrm{s} / \mathrm{he}$ falls out of the family circle.

In order to understand the role of mobile social media in Japanese families over three years we conducted fieldwork predominantly by revisiting households and viewing media practice in situ. The participants were recruited through various channels, including students from the university that the 
authors belonged to, as well as through snowballing (social capital networks from friends and acquaintances). The interviews were conducted at locations the participants were familiar with, on a one-to-one basis between one of the authors and the participants, spanning from one to two hours. Part of the process involved participants becoming familiar with the interviewer, providing a detailed understanding into the various ways social mobile media plays into everyday rhythms.

Within the interview, participants were asked to discuss - in conversation format - how they came to own their first keitai and their social media usage with their family after purchasing a sumaho. The participants themselves determined who was included in their "family." The participants were asked to bring their sumaho during the interview and to provide screen shots, to a feasible extent, of interactions between family members using social media. The participants' name (pseudonym), age, occupation, household structure, scope of "family" was assumed by the participant. In the study, eight out of twelve participants used LINE most frequently in communicating with their families (See Chapter 2). All of these eight participants were female. Over the three years of working with these families, the significance of LINE in intergenerational relationships became increasingly dominant. In addition, six out of these eight participants have a "family" group on LINE and send messages back and forth with their families. Here LINE operates as a digital genealogy for offline intimacies.

\section{Melbourne}

In Australia there were an estimated 6.4 million families consisting of a total of 19.4 million family members in 2012. The vast majority of families were coupled families $(83 \%)$ with about half of the coupled families having dependents living with them (43\%). The next largest group were one-parent families (15\%) (Australian Bureau of Statistics 2013).

One of the particularly important dimensions of Melbourne as the key city for this study is the significant number of overseas residents and, in turn, the role of social media in maintaining a sense of transnationalism. Indeed, $42 \%$ of Melbourne's residents were born overseas and the most commonly spoken language after English is Mandarin (10\%) (City of Melbourne 2013). In addition, Melbourne accommodates over 24,000 international students, including a total of 207 ancestries that residents identified with, 138 overseas countries of birth and 121 languages spoken at home other than English (Ibid.). This is reflected in our selection of families, over half of which include individuals born overseas. In addition, we actively sought out 
GLBT households (4) who are working to define and redefine the meaning of family and kinship in Australia.

In the first two phases of research in Melbourne, we utilized a method known as "tactile digital ethnography" (Pink et al. 2016), which sought to explore what the tactile and often tacit gestures around the screen articulates about mundane practices (i.e. what the body remembers); embodiment, engagement and the everyday. Tactile digital ethnography began with focusing upon filming people's hands in and around the screen while they discussed and re-enacted their locative media use. Through a focus on the hands we were able to understand their ways into the technologies and the sensory experiences that were part of this - thus opening up a route through which to gain a sense of their unspoken and often-invisible everyday technology use (Ibid.).

Over the course of interviews in families in Melbourne, a range of discussions emerged around "data" and especially discursive relationships regarding notions of privacy. In the final phase of the project we explored the ways in which people navigated data in practice, and the ways in which data, or the value of data, circulated between objects and other objects, technologies and devices, as well as between people through data and social media biographies. These often included the use of technologies and devices to "store" data such as photographs, which became traces of relationships to people or places, the transfer of devices between family members and friends with (or without) traces of data as well as the transfer of data between devices. It also attended to blockages and loss often articulated in relation to ideas about losing data, losing track of (or control of) data, and data being "trapped" on devices ranging from hard drives and smartphones. Attending to screens, the data and social media biographies were recorded via video.

By following participants over three years, Digital Media Practices in Households sought to understand media practice as dynamic and nuanced. This book outlines some of the key characteristics of media in and around familial ties. During this period, we witnessed shifts in media usage that included tensions between social media as "impression management" and self-diarization, the archival and yet ephemeral, fleeting role of media. The focus of much social media on the temporal "moment" (WeChat had even started its "Moments" feature in response to this phenomenon) leads to questions about how we can study mobile media if this trend continues, especially as there will be little or no archives for us to study. Given this trend, it became apparent that studying the gestures in and around the mobile media - that is, the way in which we frame the practices in and through 
the knowing hands-will continue to gain significance in understanding mobile media practice.

Moreover, as those participants who grew up with mobile media grow even older, their practices in and around representation of life and after-life will undoubtedly become more prevalent. So too, will issues around the curation of these mobile data trails by loved ones after they pass away. Understanding the role of stewarding (Brubaker 2016; Cumiskey and Hjorth 2018) - that is, the taking care of social media tribute pages by loved ones of the deceased-will continue to grow. Within this area, trying to make sense of tacit obligation and responsibilities will increasingly become an issue as the amount of people that pass away have digital media traces.

\section{Structure of the Book}

In this book we seek to reconcile and recalibrate the often-paradoxical relationship formed in and around continuity (kinship) and discontinuity (digital disruption). Digital Kinship allows us to think beyond the digital and through the digital historically, conceptually etc. In order to do so, this book is organized into four sections-Digital, Playful, Visualizing, and Co-futuring Kinship.

The first section, Digital Kinship, explores the ways in which kinship and forms of relatedness are being created and reproduced through digital technologies. In Chapter 2, "Platform genealogies," we consider the continuities and discontinuities around LINE, WeChat, Facebook and WhatsApp as a digital genealogy. We explore the particular histories and practices informing those platforms - what Gillespie (2015) calls "the politics of platforms" or Lamarre (2017) calls "platformativity" — and why they are being adopted intergenerationally. For example, the developed and quick uptake of LINE was in direct response to the way in which mobile social media shifted in and after the earthquake, tsunami and Fukushima nuclear disaster of 2011 known as 3/11 (Slater et al. 2012; Hjorth and Kim 2011; ITmedia Business Online 2013). The next chapter, Chapter 3, seeks to frame Digital Kinship in terms of debates around the effects of media especially in terms of emotion, intimacy and surveillance. Bringing discussions around emotion and media by Fortunati, Pertierra and Vincent (2012), along with debates around social surveillance (Humphreys 2013; Marwick 2012), mobility and transnationalism (Baldassar 2007), this chapter considers the ways that different forms of mobility (chosen and enforced) are recalibrating familial ties. 
In Section II-Playful Kinship_-we begin with Chapter 4, which revisits historical discussions around mobile media as gift (Taylor and Harper 2002) and the important anthropological meanings of the gift as a practice into power relations and rituals, to think about how we might expand upon this practice in terms of location. Here location, co-location and co-presence are revisited. In Chapter 5 we begin to focus upon the ways in which families "play" with co-presence through different engagements with digital media. We discuss in detail our methods around understanding co-present practices, especially through "tactile digital ethnography" (Pink et al. 2016). This chapter examines how studying the hand gestures in, and around, the screen might help us contextualize a more embodied practice of mobile media in the everyday.

Following upon the multi-sensorial nature of digital kinship discussed in Chapter 5 , Section III embarks on the "visual turn" within much of social mobile media. Increasingly, visual apps like Instagram are being deployed to create new forms of ambient and co-present intimacies. The first chapter of this section, Chapter 6, analyzes the growing role of the visual in social media practices in terms of tensions between sharing, impression management and self-cataloging. Chapter 7 considers the role of generational literacies and etiquettes around visual genres. For example, in our study, younger participants tended to take and share more pictures, while older participants tended to take less but comment more on their children's images. Here, generational understandings of co-present gift giving rituals can be found.

In the last section we turn to Co-futuring Kinship - the ways in which past and present practices inform how the future of the kinship for care at a distance. This is particularly important for "super-aging" contexts like Japan in which one in three is of 8 o years old. How can we map and learn from some of the informal media methods for care at a distance around supporting older adult's independence and social inclusion? Chapter 8 sets the scene for discussion around digital health in which mobile media is fully imbricated in. Discussions around a "silver bullet" in the form of a mobile app still dominant despite the fact that there is much work into the need for social, rather than technological, solutions (Gawande 2014). Chapter 9 explores how some of these practices are playing out for our participants, and how this informs generational imaginaries around data for care at a distance. In Chapter 10, we reflect upon a future rubric for imagining social mobile media.

This book therefore provides ways in which we might contextualize media practices as part of broader cultural and familial rituals. As families change, so too do their practices. Through the three locations we seek to 
provide insights into cultural and generational dynamics and the ways that locative media, as part of a datafication more broadly, can operate as a lens onto contemporary forms of kinship. We seek to explore how we can locate kinship practices in and through mobile media.

In short, locating the mobile.

\section{References}

Amagasa, Kunikazu. 2012. "Keitai to Kazoku (Keitai and Family)." In Keitaishakairon (Keitai Society), edited by Okada Tomoyuki and Matsuda Misa Hen. 101-116. Tokyo: Yuhikaku.

Australian Bureau of Statistics. 2011. "Australian Social Trends." http://tinyurl. com/bg56rdv.

Andrejevic, Mark. 2006. "The discipline of watching: Detection, risk, and lateral surveillance." Critical Studies in Media Communication 23: 391-407. DOI: 10.1080/07393180601046147.

Andrejevic, Mark, Alison Hearn, and Helen Kennedy. 2015. "Cultural studies of data mining: Introduction." European Journal of Cultural Studies 18 (4-5): 379-394.

Baldassar, Loretta, Cora Baldock, and Raelene Wilding. 2006. Families caring across borders: Migration, aging and transnational caregiving. New York: Springer.

Baldassar, Loretta. 2007. "Transnational families and the provision of moral and emotional support: The relationship between truth and distance." Identities: Global Studies in Culture and Power 14 (4): 385-409. DOI: 10.1080/10702890701578423.

Baumer, Eric, Morgan G. Ames, Jenna Burrell, Jed Brubaker and Paul Dourish. 2015. "Why study technology nonuse?" First Monday 20 (11-2), November, http:// firstmonday.org/ojs/index.php/fm/article/view/6310/5137. Accessed 10 October 2017.

Baumer Eric, Phil Adams, Vera Khovanskaya, Tony Liao, Madeline Smith, Victoria Sosik and Kaiton Williams. 2013. "Limiting, Leaving, and (re)Lapsing: An Exploration of Facebook Non-Use Practices and Experiences." Proceedings of CHI '13 Conference on Human Factors in Computing Systems. 3257-3266, http://dl.acm. org/citation.cfm?doid=2470654.2466446. Accessed 1 May 2018.

Beaulieu, Anne. 2010. "Research note: From co-location to co-presence: Shifts in the use of ethnography for the study of knowledge." Social Studies of Science 40 (3): 453-470. DOI: 10.1177/0306312709359219.

Berlant, Lauren. 1999. "Intimacy: A Special Issue." Critical Inquiry 24 (2): 281-288. www.jstor.org/stable/1344169. Accessed 10 October 2017.

Brubaker, Jed. 2016. "Stewarding." Paper presented at the International Communication Association conference, Fukuoka, June. 
Burrows, Benjamin. 2017. "YouTube Kids: The App Economy and Mobile Parenting." Social Media \& Society April-June: 1-8. DOI: 10.1177/2056305117707189.

Cabinet Office. 2013. "Shoushika shakai taisaku hakusho." (White Paper on Countermeasures to the falling birth rate society 2013). http://www8.cao.go.jp/ shoushi/shoushika/whitepaper/measures/w2013/25web honpen/html/b_s1-1. html. Accessed 5 August 2017.

Cincotta, Kate, Kate Ashford, and Katina Michael. 2011. "The New Privacy Predators." Women's Health. November.

Clark, Lyn Schofield. 2012. The Parent App: Understanding Families in the Digital Age. Oxford: Oxford University Press.

City of Melbourne. 2013. "International Student Strategy 2013-2017." www.melbourne.vic.gov.au/SiteCollectionDocuments/internat-student- strategy-2013-17. pdf. Accessed 6 August 2018.

CNNIC (China Internet Network Information Center). 2001. “第 28 次中国互联网络 发展状 况统计报告.” www.cnnic.cn/research/bgxz/tjbg/201107/t20110719_22120. html. Accessed 8 October 2018.

Daniels, Inge. 2010. The Japanese house: Material culture in the modern home. Oxford: Berg.

de Souza e Silva, Adriana, and Jordan Frith. 2012. Mobile Interfaces in Public Spaces: Locational Privacy, Control, and Urban Sociability. London: Routledge.

Dobashi, Shingo. 2006. "The gendered use of Keitai in domestic contexts." Personal, Portable, Pedestrian, edited by Mizuko Ito, Misa Matsuda, and Daisuke Okabe, 219-236. Cambridge: The MIT Press.

Dobson, Amy S. 2015. Postfeminist digital cultures: Femininity, social media, and self-representation. New York: Palgrave Macmillan.

Dourish, Paul, and Ken Anderson. 2006. "Collective Information Practice." HumanComputer Interaction 21 (3):319-342. DOI: https://doi.org/10.1207/s15327051hci2103_2. Eng, David. 2010. The Feeling of Kinship. Durham: Duke University Press.

Farman, Jason. 2011. Mobile Interface Theory. London: Routledge.

Fong, Vanessa L. 2004. Only hope: Coming of age under China's One-Child Policy. California: Stanford University Press.

Fors, Vaike, Sarah Pink, Martin Berg, and Thomas O'Dell. 2019. Imagining Personal Data. London: Bloomsbury Academic.

Fortunati, Leopoldina, Raul Pertierra, and Jane Vincent (eds.). 2012. Migration, diaspora and information technology in global societies. London: Routledge.

Foth, Marcus, Brynskov, Martin, and Timo Ojala. Eds. 2015. Citizen's right to the digital city: Urban interfaces, activism, and placemaking. Springer, Singapore.

Gazzard, Alison. 2011. "Location, Location, Location: Collecting Space and Place in Mobile Media." Convergence 17 (4): 405-417. DOI: 10.1177/1354856511414344. 
Gillespie, Tarleton. 2015. "Platforms Intervene." Social Media + Society April-June: 1-2. DOI: 10.1177/2056305115580479.

Goffman, Erving. 1959. The Presentation of Self in Everyday Life. New York: Random House.

Gold, Matthew, and Lauren Klein (eds.). 2019. Debates in Digital Humanities. Minnesota: University of Minnesota Press.

Gou, Youde. 2011. “Urbanisation and Disease Patterns in Shanghai." https://lsecities. net/media/objects/articles/urbanisation-and-disease-patterns- in-shanghai/ en-gb/. Accessed 1 October 2019.

Hasan, Helen, and Henry Linger, H. 2016. "Enhancing the wellbeing of the elderly: social use of digital technologies in aged care." Educational Gerontology 42, 749-757. DOI: 10.1080/03601277.2016.1205425.

Herzfeld, Michael. 1997. Cultural Intimacy. London: Routledge.

Hjorth, Larissa. 2007. "Snapshots of Almost Contact." Continuum 21 (2): 227-238. DOI: $10.1080 / 10304310701278140$.

- 2009. Mobile Media in Asia-Pacific. London: Routledge.

Hjorth, Larissa, and Kyoung-hwa Kim. 2011. "The mourning after: A commentary on crisis management in Japan post 3.11." Television \& New Media Journal 12 (6): 552-559. DOI: https://doi.org/10.1177/1527476411418351.

Hjorth, Larissa, and Michael Arnold. 2013.Online@Asia-Pacific. London: Routledge. Hjorth, Larissa, and Sarah Pink. 2014. "New visualities and the digital wayfarer: Reconceptualizing camera phone photography and locative media." Mobile Media \& Communication 2 (1): 40-57. DOI: 10.1177/2050157913505257.

Hjorth, Larissa, Ingrid Richardson, and William Balmford. 2016. "Careful surveillance and pet wearables: At home with animals." The Conversation, Sept 5, https://theconversation.com/careful-surveillance-and-pet-wearables-at home-with-animals-63883. Accessed 10 July 2017.

Hobson, M. and Marvin, S. 2017. "Intensifying or transforming sustainable cities?" Local Environment 1-15. DOI: 10.1080/13549839.2017.1306498.

Horst, Heather, and Daniel Miller. 2012. "Normativity and materiality: A view from digital anthropology." Media International Australia 145 (1): 103-111.

Humphreys, Lee. 2013. "Mobile social networks and surveillance: Users' perspectives." In Media, surveillance, and identity: A social perspective, edited by André Jansson and Miyase Christensen. New York: Peter Lang.

ITmedia Business Online. 2013. "LINE, Naver matome ha naze tsuyoinoka? (Why LINE and Naver are strong?)." http://bizmakoto.jp/makoto/articles/1309/o4/ newso16_2.html. Accessed 4 August 2015.

Ito, Mizuko, and Daisuke Okabe. 2005. "Intimate visual co-presence." Paper presented at Ubicomp, Takanawa Prince Hotel, Tokyo, Japan, 11-14 September. 
Jamieson, Lynn. 2011. "Intimacy as a concept: Explaining social change in the context of globalisation or another form of ethnocentricism?" Sociological Research Online 16(4): 15. http://www.socresonline.org.uk/16/4/15.html. Accessed 10 July 2017.

Kitaoji, Hironobu. 1971. "The structure of the Japanese family." American Anthropologist 73: 1036-1057.

Koch, S. 2010. "Healthy aging supported by technology." In Informatics for Health and Social Care 35, 81-91.

Lamarre, Thomas. 2017. "Platformativity." Asiascape: Digital Asia 4(3): 285-305. DOI: https://doi.org/10.1163.

Lasén, Amparo, and Elena Casado. 2012. "Mobile telephony and the remediation of couple intimacy." Feminist Media Studies 12 (4): 550-559. DOI: 10.1080/14680777.2012.741871.

Leaver, Tama. 2017 "Intimate Surveillance: Normalizing Parental Monitoring and Mediation of Infants Online." Social Media + Society 3(2): n.p. DOI: 10.1177/2056305117707192.

Lewis, Tania, Fran Martin, and Wanning Sun. 2016. Telemodernities. Durham: Duke University Press.

Lüders, Marika. 2008. "Conceptualizing Personal Media." New Media and Society 10 (5): 683-702. DOI: 0.1177/1461444808094352.

Marwick, Alice E. 2012. "The public domain: Social surveillance in everyday life." Surveillance and Society 9 (4): 378-393. https://ojs.library.queensu.ca/index. php/surveillance-and-society/article/view/pub_dom. Accessed 5 March 2016

Matsuda, Misa. 2009. "Mobile Media and the Transformation of Family." Mobile technologies: From Telecommunication to Media, edited by Gerard Goggin and Larissa Hjorth. 62-72. London: Routledge.

Ministry of Health, Labor and Welfare. 2013. "Heisei 25 nendoban kouseiroudou hakusho (White Paper on Health, Labor and Welfare 2013)." http://www.mhlw. go.jp/wp/hakusyo/kousei/13/dl/1-02-2.pdf. Accessed 26 August 2014.

Morley, David. 2003. "What's 'home' got to do with it? Contradictory Dynamics in the Domestication of Technology and the Dislocation of Domesticity." European Journal of Cultural Studies 6 (4): 435-458. DOI: 10.1177/13675494030064001.

Muramatsu, N and Yarime, M. 2011. "Japan: super-aging society preparing for the future." The Gerontologist 51 (4): 425-432. DOI: 10.1093/geront/gnro67.

Nonoyama, Hisaya. 2009. Ronten Handbook Kazoku shakaigaku (Handbook of Family Sociology). Kyoto: Sekaishisosha.

Our Mobile Planet. 2012. “Our Mobile Planet.” http://www.thinkwithgoogle.com/ mobileplanet/en/. Accessed 21 May 2012.

Pink, Sarah. 2015. Doing Sensory Ethnography. London: Sage. 
Pink, Sarah, Elisenda Ardèvol and Dèbora Lanzeni. 2016. Digital Materialities. London: Bloomsbury.

Prøitz, Lin. 2007. “The Mobile Phone Turn.” PhD Thesis, University of Oslo: Faculty of Humanities Unipub.

Ruckenstein, Minna, and Natasha Dow Schüll, 2017. "The Datafication of Health," Annual Review of Anthropology 46: 261-278. DOI: 10.1146/annurev-anthro-102116041244.

Satchell Christine, and Paul Dourish. 2009. "Beyond the User: Use and Non-Use in HCI." Human Computer Interaction 366: 9-16. https://www.ics.uci.edu/ djp3/ classes/2012_01_INF134/papers/nonuse-ozchi.pdf. Accessed 5 October 2018.

Sengupta, Somini. 2012. “Big Brother'? No, It's Parents." New York Times, 25 June, http://tinyurl.com/aysm5ud. Accessed 21 May 2013.

Sharon T, Zandbergen D. 2016. "From data fetishism to quantifying selves: selftracking practices and the other values of data." New Media \& Society 19 (11): 1695-1709. DOI: 10.1177/1461444816636090.

Sheller, Mimi. 2004. "Mobile publics: Beyond the networked perspective." Environment and Planning D: Society and Space 22 (1): 39-52. DOI: 10.1068/d324t.

Slater, David H., Keiko Nishimura, and Love Kindstrand. 2012. "Social media, information and political activism in Japan's 3.11 crisis." The Asia-Pacific Journal, Japan Focus 10 (24): n.p. http://apjjf.org/2012/10/24/David-H.-Slater/3762/article. html. Accessed 5 October2018

Statistics and Information Department, Ministry of Health, Labor and Welfare. 2012. "Graph de miru setai no joukyou (Graphical Review of Japanese Household)." www.mhlw.go.jp/toukei/list/dl/20-21-01.pdf. Accessed 30 June 2014.

Taylor, Alex S., and Richard Harper. 2002. "Age-old practices in the 'New World': A study of gift giving between teenage mobile phone users." Proceedings of the SIGCHI Conference on Human Factors in Computing Systems 439-446. New York: ACM. DOI: 10.1145/503376.503455.

van Dijck, José. 2014. "Datafication, dataism and dataveillance: big data between scientific paradigm and ideology". Surveillance \& Society 12 (2): 197-208.

Wallis, Cara. 2013. Technomobility in China: Young migrant women and mobile phones. New York: New York University Press.

Warner, Michael. 2002. "Publics and Counterpublics." Public Culture 14 (1): 49-90. DOI: 10.1215/08992363-14-1-49.

$\mathrm{Xu}$, Anqi, and Xia, Yan. 2014. "The changes in mainland Chinese families during the social transition." Faculty Publications, Department of Youth \& Family Studies. http://digitalcommons.unl.edu/famconfacpub/91. Accessed 10 July 2017. 

Section I

\author{
Digital Kinship
}





\title{
2. Platform Genealogies
}

\begin{abstract}
In Chapter 2, "Platform genealogies," we consider the continuities and discontinuities around LINE, WeChat, Facebook and WhatsApp as a digital genealogy. We explore the particular histories and practices informing those platforms - what Gillespie (2015) calls "the politics of platforms" or Lamarre calls (2017) "platformativity" — and why they are being adopted intergenerationally. For example, the developed and quick uptake of LINE was in direct response to the way in which mobile social media shifted in and after the earthquake, tsunami and Fukushima nuclear disaster of 2011 known as $3 / 11$.
\end{abstract}

Keywords: Platforms; LINE; WhatsApp; QQ; 3/11

In this book we explore the familial practices that shape and are shaped by social mobile media. Within different contexts-cultural, social, generational and familial - we can begin to de-naturalize the ways in which platforms create different affordances and practices. As we argue, through the negotiation of boundary work by families, mobile social media use and non-use is recalibrated. Rather than being platform-specific, this book considers some of the enduring practices and cultures that move in, across, and through the various platforms our participants used. As noted in Chapter 1, particular platforms dominant specific cultural contexts - for example, LINE in Japan, and WeChat in China.

The ways in which the affordances of platforms shape how people manage familial relations is significant - thus it is important to acknowledge that platforms come with their own interfaces that play a significant role in their successful uptake. Scholars such as Ian Bogost and Nick Montfort (2009) have been key in establishing the field of platform studies as an investigation of hardware and software design and the "creative works" produced on and by those systems. Their studies take into account the cultural dimensions

Hjorth, L., K. Ohashi, J. Sinanan, H. Horst, S. Pink, F. Kato, B. Zhou, Digital Media Practices in Households: Kinship through Data. Amsterdam: Amsterdam University Press, 2020 DOI 10.5117/9789462989504_CHO2 
of designed platforms, but these are often secondary to the technical and political economy dimensions.

As Tarleton Gillespie $(2010,359)$ observes in his study, discourses of specific platforms might "matter as much for what they hide as for what they reveal" He argues that these uses connote the concept-literal or metaphorical — of an expansive, level, nonhierarchical surface, hosting multiple activities or events. Platforms thus "anticipate" certain actions, implying a certain neutrality or egalitarian organization that provides an open or democratic space supporting users (individuals or corporations) (Ibid., 350). Such metaphors echo the rhetoric of Web 2.0 as a place facilitating user-created content, amateur creativity, production, creative practice and networking. Yet, as Gillespie demonstrates, the "comforting" ideas of free space and openness obfuscates the tensions and power relationships between the roles and actions of providers, users, and commercial media companies in the creation, distribution, and controlled delivery of online content. José van Dijck builds on Gillespie's discussion by arguing that we live in a "platform society" whereby platforms are "gateways" for everyday social life (2016). Understanding platforms as part of a genealogy means taking seriously the relationality of platforms and how they are part of a broader cultural, social and historical practice. As Hjorth et al. suggest:

We consider platforms as emerging as places in which the digital and material are entangled, and we account for how platforms are made through intensities of social engagement as much as through their occupation of physical sites. Platforms become central to thinking about the clustering of experiential and representational forms, as part of places that are made up of things, persons, and processes of different qualities and affordances, and as open, continually changing aspects of the environments we inhabit $(2016,31)$.

In this book we use the concept of genealogy as means of tracing of lines of descent, lines being an important concept for understanding the entanglement of bodies with the digital and material. For anthropologist Tim Ingold, "the entanglement of things has to be understood literally and precisely, not as a network of connections but as a meshwork of interwoven lines of growth and movement" (2010, n.p). Meshwork highlights the biographies, relations, and participation of objects, people and media.

Michel Foucault (2003) used the concept as a philosophical historical technique in which social beliefs are put into context. His work was particularly interested in power and the different way knowledges come into 
existence. Framing platforms in terms of genealogy allow for continuities and discontinuities, in which platforms as seen as transitory in the digital kinship lines of descent. Here we view platforms as biographies for practices. Given the significance of lines in many of the definitions of genealogy, this chapter will begin with one of the key platforms used in Japan, LINE. We then discuss WeChat in Shanghai and then Facebook, WhatsApp and Instagram in Melbourne. We conclude by discussing one of the enduring continuities across the three sites and multiple platforms-especially in terms of paralinguistics such as emojis ("picture characters"), emoticons (typographic characters), stamps and stickers.

\section{Japan: LINE: A Post 3/11 Social Media}

The social mobile media app LINE has become popular in Japan due to the particular technological, cultural, social, and linguistic factors informing the uptake and adoption of social and mobile media. As mentioned in the introduction, the success of LINE emerged in direct response to the way in which mobile social media shifted in and after the disaster of $2011(3 / 11)$ - as a result of declining trust in traditional media such as NHK (Hjorth and Kim 2011; Slater et al. 2012; ITmedia Business Online 2013; Steinberg 2019). The ex-president of LINE, Mr. Morikawa, noted that they decided to develop LINE because they saw many people use Twitter as a communication tool between intimates at the time of 3/11 (ITmedia Business Online 2013). LINE quickly became embedded in everyday social media practices.

Post 3/11 social mobile media-especially LINE — became integral to many people's everyday life and interpersonal relationships, especially for family communication. Some have suggested that LINE is the form of intergenerational communication for Japanese by enveloping mundane intimacies with hybrid forms of new media literacy. The digital genealogies of LINE reflect offline familial intimacies that both continue older familial rituals while also providing new ways for care at a distance, accounting for what Mizuko Ito and Daisuke Okabe (2005) have called intimate ambient co-presence. The adoption of LINE plays into broader historical practices of mobility in Japan that include long commutes as part of everyday life (Fujimoto 2005).

For Kenichi Fujimoto, understanding mobile media in Japan requires a differentiation between mobility and mobilism. Fujimoto defines mobilism as "broader cultural and social dimensions such as malleability, fluctuation and mobilization" $(2005,80)$. Unlike mobilism, "mobility has tended to refer to functional dimensions of portability and freedom from social and 
geographic constrain" (Ibid.). Thus, mobilism is tied to socio-geographic factors, whereas mobility infers transcendence, particularly around geographic constraints. The distinction between mobilism and mobility becomes important when considering mobile media as part of the fabric of cultural, social and geographic textures in everyday life.

In Japan we see how social mobile media enabled alternative ways to articulate the personal and intergenerational as well as the political and social. For example, immediately after $3 / 11$, reports suggest that in the central Tokyo area (including prefectures adjacent to Tokyo) more than five million people could not reach their home on that day because of the damage to public transportation. According to the survey conducted by Weather News (2014), it took about seven times longer to reach home when compared to one's ordinary commuting.

While many stayed in their office building, some people decided to walk home. During the walk home, many people tried to use Twitter to collect information about on-going changes in transportation recovery. One could, in fact, spontaneously change the route toward his/her home based on communication through tweets. Social media enabled coordination at a micro level. The experience of the earthquake motivated people to utilize social media applications to gather and share information about changes in the social and political climates (Slater et al. 2012; Gill, Steger and Slater 2013).

In addition, mobile media operated symbolically as a vessel for containing the intimate during times of grief - some people held onto their phones as security when they couldn't make contact with loved ones (Hjorth and Kim 2011). In particular, the rise of social mobile media like LINE in Japan highlights the significance of the media in maintaining and fostering intergenerational ties. These are personal as they are political; intimate, as they are public.

LINE remains an integral part of the social media landscape in Japan and its significance is marked by a cross-generational use, and in this way can be viewed as a metaphor for familial genealogies. LINE is a service providing text messaging among individuals or groups, as well as making phone and video calls for no charge. The Stamp function-an advanced form of paralinguistics such as emoji (pictogram or emoticons) and decomail-allows communication without the need of text messaging and the kawaii (cute) culture was key to its success. These functions allow for more affective care at a distance activity, providing co-present gestures of emotion and feeling that can be seen to transcend words and be more sensitive to social context than just words.

Byford (2013) notes that LINE in Japan has introduced the "next-level emoji" through their deployment of stickers (stamps). As we explore later, 


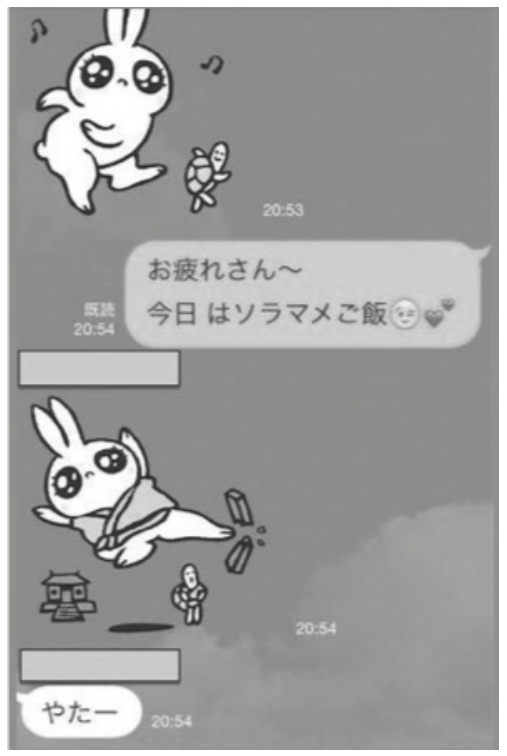

Figure 2.1: Chat with rabbit stamp on LINE

Stark and Crawford $(2015,1)$ define stickers as "proprietary to each platform that sells them." However, the ambiguities of emoji and stickers in and across work contexts like emails and social media can confuse and also be viewed as part of broader problematic of affective labor practices. In the case of families dealing with distance, they can provide the intimacy and care at a distance that is essential for maintaining intergenerational relationships.

In Japan, keitai cultures have afforded ways in which to express forms of intimacy previously left tacit (Ito, Matsuda and Okabe 2005). As noted previously, kawaii (cute) culture informed the invention of emojis in Japan in 1999. Kawaii cultures play with the premature adulthood and gender conventions (Kinsella 1995; Hjorth 2003a, 2003b, 2005) and play, affect and labor are entangled in particular ways. For example, the rise of mobile media (keitai) in Japan was marked by a personalization culture both inside and outside the device (Hjorth 2009). The rise of the online saw the further development of kawaii cultures through ASCII paralinguistics, especially by high school girls who creolized hiragana with ASCII in what has now been called the high school pager revolution (Fujimoto 2005; Ito et al. 2005; Hjorth 2009). Also, there is a long history of deploying the kawaii to make friendly or "warm" the coldest of new technologies (Hjorth 2003a).

By 2015, the number of domestic registrants for LINE was 58 million, or over $45 \%$ of the whole population (LINE 2015). At this time the popularity of LINE had overtaken the role of social media like mixi, GREE 
and Mobage. Deploying kawaii characters have played an important role in Japanese culture and expressing emotions (Hjorth 2003a, 2003b). LINE thus provided the growing amount of sumaho users with easy, fun, familiar, affective and mundane ways to communicate with copresent intimates. Here the kawaii functions to personalize and bring an emotional softening to digital encounters. The kawaii helps to embed particular feelings associated with the message, thus allowing for building emotional ties.

\section{China: WeChat}

On 21 January 2011, Tencent Technology officially launched its mobile instant messaging (IM) product WeChat, with the slogan "To connect people, to connect goods, and to connect everything." Tencent Technology understood the Chinese mobile and internet market well, having established QQ, one of the most important first-generation IM mobile apps. For many generations, QQ was their first entry into mobile internet — and for many of the older generations, it was synonymous with the internet.

WeChat has undergone six major iterations, evolving from one single IM product into one of the most popular mobile apps that combines communication, social networking, media and e-banking functions together. By June 2015, it reached a record 90\% coverage of mobile phone users. In the first quarter of 2015, the number of people engaging with WeChat reached 549 million, with many suggesting that the emergence of WeChat stickers (like LINE stamps) played a key role in this rapid uptake (Tencent 2016). WeChat had developed a very young user group base, with an average age of 26 , where $86.2 \%$ were young adults between the ages of 18 and 35 .

WeChat's friendly interface includes four major interactive componentsChats, Contacts, Discover and Me. As the basic function of WeChat, Chats is set as the default home display for WeChat users. In the Chats section, private chats (with single head portrait) and group chats (with multiple head portraits) are both available for users. Users can also subscribe to public accounts according to their interests.

Multiple sub-functions can be initiated within the Chats section. WeChat allows sending texts, photos, audio, videos, locations and one's favorites. The "Red Packet" and "Transfer" function allows money to be exchanged between WeChat users. The second most-used part of WeChat is the third icon on the bottom, "Discover." In the Discover section, users can find the social function of WeChat, i.e., "Moments." 
New feeds in WeChat Moments are displayed in the informational flow style - similar to Facebook in design. Users can choose to "check-in" when sharing a feed to Moments. In the right screenshot, information in the red rectangle is a snapshot of the "check-in." The way of being social in Moments is by selecting "like" or "comment" on each post. All posts of one single user are date stamped as his or her posts, including all text, image and online content posts. Locative information is also displayed in the individual post page. In the screenshot above, information in the red rectangle is another sample of "check-in." The fourth part is the personal function of WeChat, which includes supportive functions of WeChat, for example, Wallet (for WeChat Pay).

It took WeChat over five years to grow from a simple instant messaging app to a comprehensive social and e-commerce app. However, it is significant that the broad uptake of WeChat is not necessarily represented by youth but, rather, through the engagement with multi-generational functions. In China, it is now very fashionable for families to have a family WeChat group. Like LINE in Japan, WeChat in China can be seen as an extension of kinship rituals. A family WeChat group provides an online private place for domestic communication, and this is especially useful for families when they are physically apart, as is increasingly becoming the case. The easy use and almost zero cost of WeChat makes communication affordable for everyone; the media rich function of WeChat makes communication on WeChat more vivid and multi-dimensional. WeChat brings distant family members together and creates a sense of co-presence.

25-year-old Yolanda is a key example of someone who used WeChat to extend the lines of connection between her and her family when she moved away to pursue a degree in International Relations in Shanghai. Yolanda's hometown was far from Shanghai, and so she only traveled back home to visit her parents - who worked as civil servants - three times each year. For this reason, she established a WeChat family group, and the family moved in and out of the group throughout the day to discuss everyday events, feelings and family rituals. However, Yolanda's main focus was to keep in contact with her peers on WeChat, as they were all frequent users. For Yolanda, texting was not as flexible as WeChat and lacked the full emotional expression made possible with other platforms.

Usually Yolanda's mother initiated the conversation in the family chat group, but she preferred talking to typing so rarely sent text messages, instead she favored the playful use of stickers. Yolanda told her parents that she still had a scholarship for the semester and her mother replied with a sticker saying, "So Happy." Yolanda then registered her own official account on WeChat and started writing fashion blogs on her public account. 


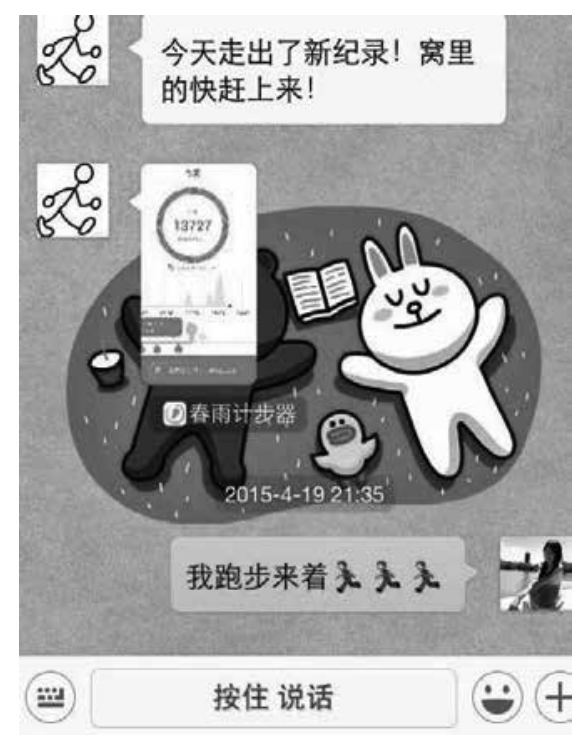

Figure 2.2: Yolanda's father sharing his daily jogging record in the family WeChat Group

Her parents subscribed to her official account to read her blogs and invited their friends to follow, which made Yolanda feel a little awkward in that she did not regard her parents' friends as her target audience. But she still appreciated her parents' passionate support.

Yolanda taught her parents how to use WeChat during the times she visited home, such as during the Spring Festival. Her mother asked about the WeChat Red Packet and so Yolanda spent time figuring out how it worked. She liked to encourage her parents to use digital media to help them discover and cultivate their interests. Considering that her parents planned to retire in 10 years, she wanted them to gain experience with digital media for their retirement. Yolanda also encouraged her parents to share in Moments of WeChat. Due to their concern for privacy, initially Yolanda's parents told her not to share too much on social media. She hoped that her parents would share more in Moments so that the family could learn more about each other by reading each other's social media posts.

\section{Melbourne: Facebook, WhatsApp and Instagram}

Unlike Shanghai and Tokyo where one platform dominates social media, social mobile media practices in Melbourne reflect what Madianou and 


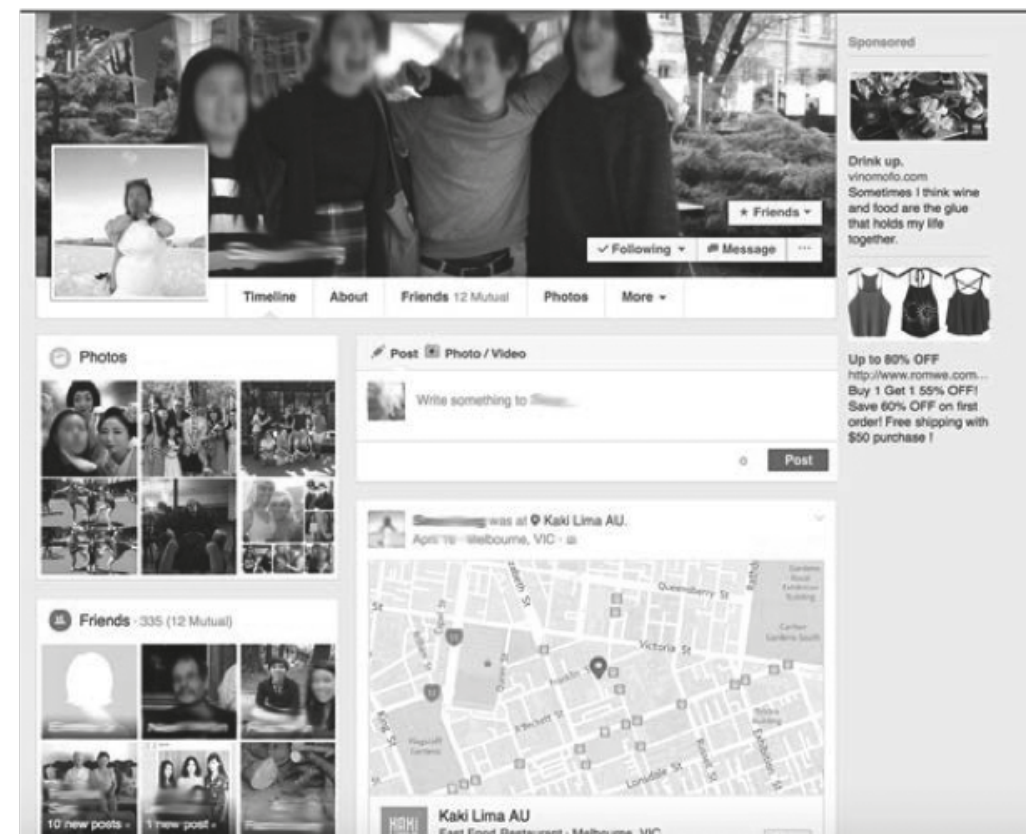

Figure 2.3: Facebook page from our Melbourne participants

Miller (2013) call "polymedia." The concept of polymedia attempts to understand the social and cultural dimensions around the use of increasingly diverse platform technologies as part of the ever-changing interpersonal communication channels. In Melbourne this includes a mix of Facebook, WhatsApp and Instagram.

According to Social Media News (2016), Facebook has 15 million Australian users. Facebook emerged as the dominant social media platform between 2007 and 2008, overtaking then popular social networking sites such as MySpace. The most prominent feature of Facebook, the "profile," operates in a similar way to a bulletin board, where users post textual updates (or "status"), images, videos, links and curated "life events"; all of which create the "timeline" or "newsfeed." Once logging on or checking Facebook from a smartphone, users see posts by other "friends" in their networks appear chronologically in their timeline.

Interaction with posts by others encourages leaving a "like" in the form of paralinguistics — clicking on a thumbs-up icon or another emotional reaction e.g. wow, sad-or by leaving a comment that can instigate a conversation. Members can also create groups, which allows those with mutual interests to share, update and discuss. They also have the ability to create events to inform, publicize, invite guests, and keep track of 
attendance numbers; to manage pages around a particular interest or business; and to private message friends within a confined space. The growth of interaction from once just a "like" to now six different reactions reflects a broader trend in the increasing personalization and affective labor in social media practices. Facebook and the linked Facebook Messenger app also have its own version of stickers. Here we can see the platform proprietary of stickers as much like a type of vernacular or local platform paralinguistics.

Wilken (2014) has also noted the growing importance of Facebook as a location-based platform since it increased locative capacities in 2010; to allow Facebook to dominate as a location-based services company, a recommendation service and to allow for more, targeted advertising campaigns. Yet, as we explore further in this book, the concerns for location, data, privacy and surveillance vary from friendly surveillance as location as known by others in one's networks and data privacy in relation to third parties, companies and government bodies.

WhatsApp has far fewer Australian users-approximately 2.4 million-and our study suggests that WhatsApp is more popular with individuals who have friends and relatives overseas. In our study this was most prominent among migrant families with connections in Southeast Asia. WhatsApp gained popularity in relation to its predecessor, BlackBerry Messenger (BBM), which could only be used between BlackBerry users. When Samsung and other Android phones became prominent competitors to the BlackBerry and iPhone markets, WhatsApp gained traction as it could be used across infrastructural platforms, between users of different smartphones.

Similar to BBM, WhatsApp replicates mobile phone text messaging functions, but uses broadband WiFi to enable unlimited "free" message exchange, phone calls and video calls. Messages accumulate in the form of a chat between two or more individuals as a group. The group chat function has emerged as an important feature throughout the fieldwork in Melbourne, for those who belong to vocational groups and for families with relatives overseas. Within messages, people can also send links, video and audio clips, and images. Image exchange has become an important way of facilitating ambient co-presence and sharing mundane aspects of everyday life as a form of bonding. Messages include a time stamp and one tick appears when the messages have been sent, two ticks appear when the message has been received and turn blue when the message has been read. Individuals can also see the last time their correspondent was online, if they are currently online, and when they are typing a message. 


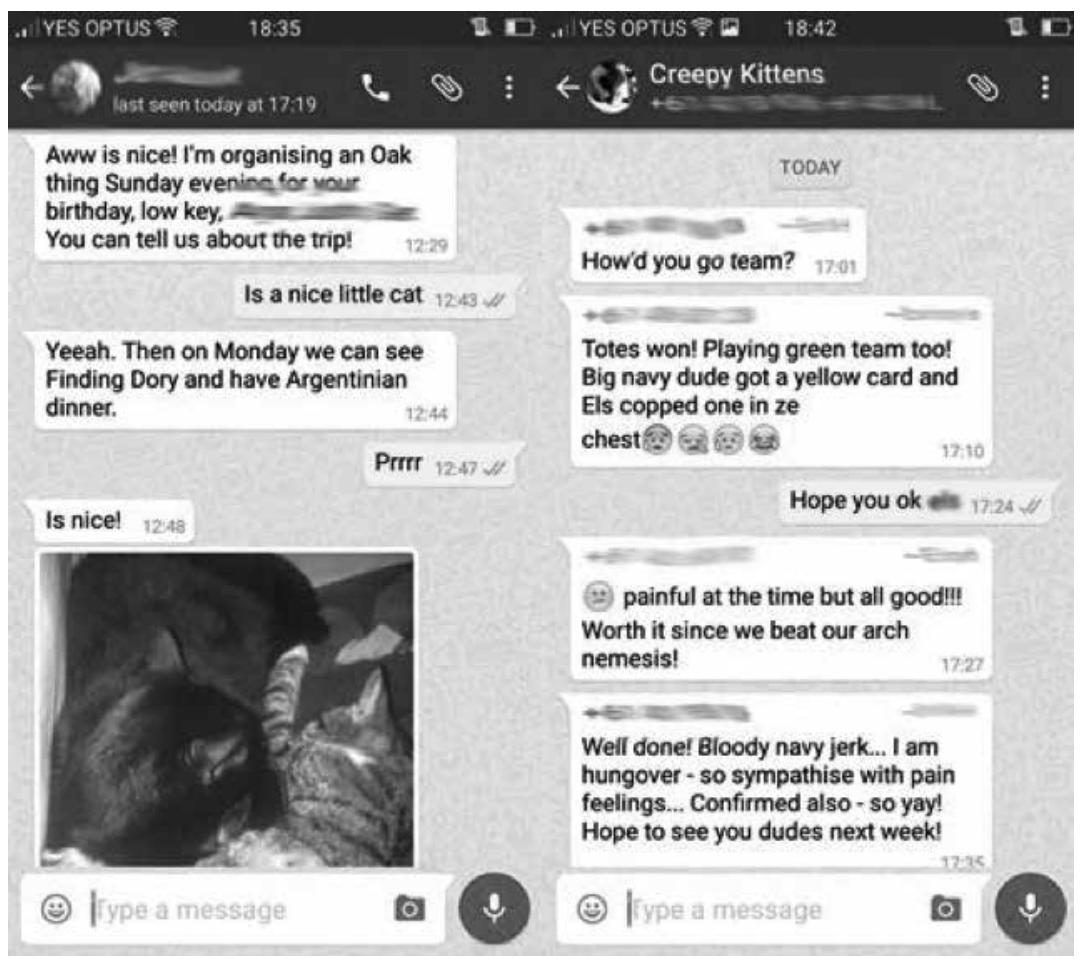

Figure 2.4: WhatsApp

WhatsApp currently does not display location data between those communicating although location data is collected through the platform as accessed on a smartphone. One of the main uses of WhatsApp is the use of groups. This function challenges popular arguments that social media facilitates ego-centered networking. Throughout our fieldwork in Melbourne, group chats were used in a variety of ways. Take 25 -year-old Jasmine as an example. She joined a family group chat, consisting mostly of relatives overseas after a cousin had a baby. The cousin posted images of her newborn every day and different relatives would comment, often resulting in an ongoing conversation. Jasmine herself did not respond often to the chat messages but liked being included as a "lurker" who could read and listen to the messages. Other participants were far more active within group chats.

30-year-old Lily was involved in two WhatsApp groups, one with her close friends from church and the other a mothers' group. The mothers' group exchanged recipes and photos of dishes they were preparing at least a few days a week. When we visited Lily, it was obvious her passions were cooking and baking. She had a bookshelf of cookbooks, exercise books with 
handwritten recipes, and recipes she had printed out from the internet. She also had a food app on her phone that allowed her to browse and share recipes. The WhatsApp group was yet another way for Lily to collect, store and share recipes.

WhatsApp groups were also used between extended family members living overseas for coordinating vocational interest groups. Daniel, for example, was in his late 5os and an active member of a men's choir. They had a WhatsApp group chat to update each other of rehearsal schedules and upcoming events, and exchanged news links and some banter through the week between seeing each other.

Similarly, 6o-year-old Esther and 57-year-old Stephen were a brother and sister who moved to Australia with their families. Since joining Facebook and WhatsApp, they had become far more active within group conversations, mostly on WhatsApp. Esther socialized in a group with her old high school friends from the 1970s, which had over 60 members. Esther received several picture messages every few weeks. She noted that she routinely deleted images that involved politics or anything crude. She kept old photos that her friends scanned and sent, memes of greetings or with sayings she found positive or motivational, and holiday photos. She manually backed up images sent over WhatsApp from her phone to her laptop every few months. Esther found that rekindling a relationship with her old friends from Malaysia was a pleasant and relaxing distraction at the end of her workday.

Like Esther who socialized in WhatsApp with old friends, Stephen participated in a WhatsApp group with his ex-navy colleagues where they exchanged family and travel photos. They also sent funny memes or images that Stephen kept on his phone and looked at from time to time. Stephen also administered his ex-navy colleagues' Facebook page, but he found that the group posted minimally on the page because the WhatsApp group chat was so active. Family in these WhatsApp groups were the main content that members shared.

\section{Locating the Platforms Across the Sites: Emojis/Stamps/Stickers}

In each of the locations, uptake of specific platforms operates in relation to familial relationships. Participants shaped the platform to perform particular forms of affect and co-present intimacy. Across all the different platforms discussed, emojis/stamps/stickers played an important role in everyday mundane rituals - especially when physically co-located. Here we see the playful characteristic of contemporary media come to the forefront (Sicart 
2014). The use of emoticons - emoji-has become an increasingly popular and playful way of personalizing digital media communications.

As one Japanese participant 34-year-old Mana noted, she shared lots of stamps to send love to geographically distant family members. For Mana, a great deal of time was spent using creatively situational stamps, such as "Cute!" and "Take care!" to create a sense of participation and being there. The stamps gave a sense of feeling and emotional that can be hard to express in words.

As discussed earlier, in Asian countries such as Japan, the historical trajectories towards emoji use are tied to the role of the kawaii. The historical context in Japan offers a starting point for considering how such practices have come about and suggests that we would likewise find similar but differently visible forms of cute personalization in other cultures. We have found that with the now ubiquitous (albeit uneven) uptake of mobile social media in the Asia-Pacific region, localized forms of cute character culture have become prevalent across cultures and intergenerationally. Our research has shown how grandparents, parents and children across our Australian, Japanese and Chinese research sites, can all be found deploying different forms of paralinguistics-emojis, emoticons and social media stickers (i.e. LINE, WeChat, WhatsApp) —in playful ways that are tied to generational norms, as well as providing new ways to transgress intergenerational boundaries. We argue that paralinguistics like emoji and stickers can provide an affective care at a distance and co-present intimacy that not only helps to bind families when they are experiencing geographic distance but also provides playful and creative ways for intergenerational literacy and lasting connection.

Through the three different case studies described, we can see the role of paralinguistics in sustaining intimacy intergenerationally-especially when distance is an issue. As we saw in the case of Japan and China, young adults teach their parents to use paralinguistic methods when they move away from home as a way to keep constant contact. In Japan and Australia, the usage between mothers and daughters dominate. Whereas in Australia, it is predominantly migrant participants who use paralinguistics the mostespecially for keeping in close contact with young family members abroad.

Paralinguistics, we suggest, provide a space to explore emotions in ways that traditional forms of language in media platforms cannot. Entangled within everyday mundane digital media practices of care (see McKay 2007; Yeates 2004; Lynch and McLaughlin 1995), our insights into the uses of and feelings that are generated through paralinguistics build on research around family members "do family" at a distance through everyday activities that 
extend beyond the home including through digital media (Morgan 1996; Wajcman et al. 2008; Madianou 2016).

Recent literature in fields such as social media have started to take emojis seriously, as part of the affective labor landscape and playbour of contemporary digital media (Stark and Crawford 2015). Work and young people have been the primary focus, leaving little research exploring how these affective practices play out within families, especially in terms of families grappling with physical distance. As we see in these three localities, the power of emojis have what Stark and Crawford $(2015,1)$ describe as "conduits for affective labor in the social networks of informational capitalism" become central. Different generations, cultures and labor settings are all deploying the emoji with numerous affective and creative practices. As Stark and Crawford note, emoji help to creatively manage "everyday biopolitics":

The patterns of use for emoji over time between friends and partners can become abstract and cryptic, or can degenerate to become pro forma-just plain basic. In the best case, there is a unique personal subtext to that exchange of a rainbow or the love-heart smile, many layers of unspoken meaning that would be difficult for intelligence analysts or machinelearning algorithm to parse. Nonetheless, this complexity has not stopped institutions from making the attempt, and commercializing emoji sociality in other ways $(2015,6)$.

Paralinguistics, we argue, can be seen as an extension of what Julian Kücklich (2005) called "playbour" - that is, involving emotional, creative and social labor in and around their playfulness. In turn, paralinguistics identify a fundamental paradox underscoring digital labor - that is, it is both a playground and a factory (Scholz 2012). Comparing their use in Shanghai, Tokyo and Melbourne with families, we found some of the vernaculars around paralinguistics including "stamps" help with managing intimacies at a distance. In our research with families where adult children lived away from their parents, paralinguistics and stickers were viewed as an important part of the emotions at a distance, by creating a type of affective warmth that sometimes over exceeded face-to-face contact and intimacy.

We argue that paralinguistics like emoji and stickers can provide an affective care at a distance (continuity) and co-present intimacy that contributes in playful and creative ways to intergenerational literacy and connection. As extensions of historical tropes like the kawaii, emojis help to provide emotional warmth around "cool" new media. This chapter has taken the first steps towards understanding this phenomenon. 
We end by proposing that since paralinguistics appear to be here to stay, at least for the immediate future, and the role that they play in everyday mundane and affective lives needs further ethnographic investigation. This is important since such a research agenda seeks to reveal not simply how and why paralinguistics are important in the affective lives of families, but also suggests how such intergenerational relationships are bound up in the problematic global political economies of digital media, technology and labor that are inseparable from this context. In the next chapter we explore the role of locative media, as an integral part of social mobile media, in recalibrating forms of what we call "friendly surveillance." Like the emotional labor of emojis/stickers/stamps, the use and non-use of locative media provides families with ways in which to make and unmake boundaries of intimacy and kinship.

\section{References}

Bogost, Ian, and Nick Montfort. 2009. "Platform Studies." www.platformstudies. com/. Accessed 21 May 2015.

Byford Sam. 2013. "Path moves toward monetization with stickers and private messaging in 3.o." The Verge. www.theverge.com/2013/3/6/4073002/path-3-0update-available-with-messaging-and-shop. Accessed 21 May 2015.

Foucault, Michel. 2003. The essential Foucault: Selections from essential works of Foucault, 1954-1984. New York: The New Press.

Fujimoto, Kenichi. 2005. “The third stage paradigm.” Personal, portable, pedestrian: Mobile phones in Japanese life, edited by Mizuko Ito, Misa Matsuda, and Daisuke Okabe. 77-102. Cambridge: The MIT Press.

Gill, Tom, Brigitte Steger, and David H. Slater. 2013. Japan copes with calamity. New York: Peter Lang.

Gillespie, Tarleton. 2010. "The Politics of 'Platforms'." New Media \& Society 12: 347-364. DOI: 10.1177/1461444809342738.

Hjorth, Larissa. 2003a. "Kawaii@keitai." Japanese Cybercultures, edited by Nanette Gottlieb and Mark McLelland, 50-59. New York: Routledge.

—. 2003b. "Pop and Ma." Mobile Cultures, edited by Fran Martin, Audrey Yue, and Chris Berry, 158-179. Durham: Duke University Press.

- 2005. "Odours of mobility." Journal of Intercultural Studies 26 (1-2): 39-55.

- 2009. Mobile Media in Asia-Pacific. London: Routledge.

Hjorth, Larissa, and Kyoung-hwa Y. Kim. 2011. "The mourning after: A commentary on crisis management in Japan post 3.11." Television \& New Media Journal 12 (6): 552-559. DOI: 10.1177/1527476411418351. 
Hjorth, Larissa, Sarah Pink, Kristen Sharp, and Linda Williams. 2016. Screen Ecologies. Cambridge:The MIT Press.

Ingold, Tim. 2010. "Bringing things back to life: Creative entanglements in a world of materials." NCRM Working Paper. Realities / Morgan Center, University of Manchester (Unpublished). http://eprints.ncrm.ac.uk/1306/. Accessed 21 May 2015. ITmedia Business Online. 2013. "LINE, Naver matome ha naze tsuyoinoka? (Why LINE and Naver are strong?).” http://bizmakoto.jp/makoto/articles/1309/o4/ newso16_2.html. Accessed 4 August 2015.

Ito, Mizuko, Misa Matsuda, and Daisuke Okabe. eds. 2005. Personal, portable, pedestrian: Mobile phones in Japanese Life. Cambridge: The MIT Press.

Ito, Mizuko, and Daisuke Okabe. 2005. "Intimate visual co-presence." Paper presented at Ubicomp, Takanawa Prince Hotel, Tokyo, Japan, 11-14 September.

Kinsella, Sharon. 1995. "Cuties in Japan." In Women, Media and Consumption in Japan, edited by Lisa Skov and Brian Moeran, 220-54. London: Curzon Press.

Kücklich, Julian. 2005. "Modders and the games industry." Fibreculture Journal Issue 5. http://five.fibreculturejournal.org/fcj-025-precarious-playbour-moddersand-the-digital-games-industry/. Accessed 4 August 2015.

LINE. 2015. "2015 nen 4 gatsu - 9 gatsu baitai shiryou (LINE media guide 2015 AprSep)." https://linecorp.com/ads/pdf/8CCCEF52-B730-11E4-BEB8-ED3 AF5 F15 F22. Accessed 4 August 2015.

Madianou, Mirca, and Daniel Miller. 2013. "Polymedia: Towards a new theory of digital media in interpersonal communication." International Journal of Cultural Studies 16 (2): 169-187. DOI: 10.1177/1367877912452486.

Scholz, Trebor. Ed. 2012. Digital labour: The internet as playground and factory. New York: Routledge.

Sicart, Miguel. 2014. Play Matters. Cambridge: The MIT Press.

Slater, David H., Keiko Nishimura, and Love Kindstrand. 2012. "Social media, information and political activism in Japan's 3.11 crisis." The Asia-Pacific Journal, Japan Focus 10 (24):1-33. https://apjjf.org/2012/10/24/David-H.-Slater/3762/article. html. Accessed 21 May 2015.

Social Media News. 2016. "Social Media Statistics - August 2015." http://www. socialmedianews.com.au/social-media-statistics-australia-august- 2015/. Accessed 21 May 205.

Stark, Luke, and Kate Crawford. 2015. "The conservatism of emoji: Work, affect, and communication." Social Media \& Society July-December.1-11. DOI: $10.1177 / 2056305115604853$.

Tencent. 2016. “2016 Annual Report.” http://www.tencent.com/zh-cn/content/ir/ rp/2016/attachments/201601.pdf. (In Chinese). Accessed 2 May 2017.

van Dijck, José. 2016. "Public Values in a Platform Society.” AoiR keynote, Berlin October 2016. 
Weather News. 2011. "A survey on "stranded commuters." Tokyo Metropolitan area on the day of Great East Japan Earthquake, 11 March 2011. http://weathernews. com/ja/nc/press/2011/110411_2.html. Accessed 31 October 2015.

Wilken, Rowan. 2014. "Proximity and Alienation." The Mobile Story: Narrative Practices with Locative Technologies, edited by Jason Farman, 175-191. New York: Routledge. 



\title{
3. Friendly Social Surveillance
}

\begin{abstract}
Chapter 3 seeks to frame Digital Kinship in terms of debates around the effects of media especially in terms of emotion, intimacy and surveillance. Bringing discussions around emotion and media by scholars, along with debates around social surveillance, mobility and transnationalism, this chapter considers the ways that different forms of mobility (chosen and enforced) are recalibrating familial ties.
\end{abstract}

Keywords: intimacy; surveillance; families; transnationalism

32-year-old Maki and her mother 57-year-old Eriko lived in a town 30 minutes train ride from the center of Tokyo. For Maki and Eriko, setting up Facebook events was a regular part of their jobs. When they organized events, they wrote announcements with pictures and location information, and posted them on Facebook. They read each other's posts and didn't post private information on Facebook. For Maki, Facebook facilitated a type of social surveillance: "Since we started to use Facebook, we know each other's situation without talking." By contrast, they described LINE as "a very private tool. We use Facebook for making announcement to the public."

While much of the surveillance literature has focused upon corporate or governmental dimensions of social media, a range of other kinds of surveillance is apparent in everyday practice. These can be horizontal and vertical, benevolent and malevolent, and reflect long-standing practices of gossip, monitoring and even parenting. As Maki and Eriko described, LINE creates a private and individuated mode of co-presence and social surveillance, while Facebook is for more collective and public social surveillance. These practices reflect new forms of social surveillance (Marwick 2012) within families that are creating an additional — and to date under-researched-layers of complexity (Clark 2012; Sengupta 2012).

Despite the continuity with previous practices of social surveillance, we still know very little about how privacy, intimacy and surveillance are being

Hjorth, L., K. Ohashi, J. Sinanan, H. Horst, S. Pink, F. Kato, B. Zhou, Digital Media Practices in Households: Kinship through Data. Amsterdam: Amsterdam University Press, 2020 DOI 10.5117/9789462989504_CHO3 
played out in everyday family contexts through locative media, or the ways in which these dynamics impact how, when and where locative media are used. We also know little about how place and time shape these experiences. As we have explored in previous chapters, different forms of intimacy and kinship weave their ways through media practices. In these practices it is the social or lateral dimensions of surveillance that best encompass the paradoxes of care in and around technology, and data in the home. Here we see care as a texture, contour and practice, moving in and out of the daily rhythms. Care is a complex layering of emotion and slowness that is often entangled with practices such as surveillance.

Alice Marwick distinguishes "social surveillance" from traditional forms through three axes_-power, hierarchy and reciprocity (2012, 378). Utilizing Foucault's notion of capillaries of power, Marwick argues that social surveillance assumes "power differentials evident in everyday interactions rather than the hierarchical power relationships assumed in much of the surveillance literature." Marwick identifies some of the common notions of surveillance such as lateral (Andrejevic 2006), participatory (Albrechtslund 2008) and social (Ellison, Steinfeld and Lampe 2007; Joinson 2008; Tokunaga 2011). As she notes, social surveillance differs from traditional models insofar as it is focused around micro-level, de-centralized, reciprocal interactions between individuals. Marwick frames her definition in terms of boundary work (Nippert-Eng 2010, 10-14) whereby privacy is not necessarily framed by dichotomies of divisions across spatial, temporal and object-related work (Marwick 2012, 379).

Datafication of everyday life complicates surveillance. With tracking data on devices from phones to wearables, two key camps have emerged. Those that recognize the ways in which the data is used by corporation in ways that users are yet to understand. In recent years, especially through corporate data harvesting such as the Cambridge Analytica debacle, it has become apparent that there is a need for more robust regulation. The Facebook-Cambridge Analytica data scandal happened early in 2018 when it was revealed that Cambridge Analytica used millions of Facebook profiles without their consent for political gain. And while in Europe there has been the rollout of the European General Data Protection Regulations (GDPR) in May 2018, there are concerns about companies such as Google and Facebook not being accountable.

Artists and creatives such as Egor Tsvetkov have been making devices or using facial recognition applications like FindFace to demonstrate dystopian realities. In Tsvetkov's Your Face is My Data (2016) photographs of strangers on the Russian subway where identified via their social media profile by the facial recognition app FindFace. Another example can be found in the 
Trevor Paglen and Kate Crawford's viral art \#ImageNetRoulette project which subverted a leading facial recognition database to remove more than half a million images. The project sought to make people aware of the facial recognition biases and to change people's minds about AI (2019). The project resonates with a subgenre of artists working to creatively subvert social and mobile media technology to make more public awareness. Other creatives such as wearable artist Camille Baker are using wearables to bring attention to non-normative bodies (2017). These practices bring to question the interrelationship between privacy, data and trust and how we might gain empowerment beyond corporate exploitation. In the space of digital health and self-tracking, the work of Lupton has been crucial in identifying some of the issues around dataveillance (Lupton 2016). As Albrechtslund (2008) notes, "participatory surveillance" involves active surveillance of self and others in ways that are productive and social.

In our fieldwork across the three sites families and intimates created their own types of friendly surveillance. From the locative function on Facebook that allows friends to monitor, to parents ambiently watching their children's relationships through WhatsApp, mobile media is providing creative and playful ways to manage intimate intergenerational relations at a distance. In this chapter we reflect upon the different forms of cross-cultural intergenerational friendly surveillance (care at a distance or co-present care). We begin with a discussion of debates around surveillance before exploring the many ways co-present care plays out in everyday scenarios across Tokyo, Shanghai and Melbourne.

\section{Understanding Contemporary Surveillance: A Familial Model}

With the rise of mobile technologies, what constitutes surveillance has diversified. As we noted in Chapter 1, there are some key trajectories for understanding the rise and diversification and localization of surveillance. We pointed to the work of Humphreys (2013) who outlines a specific form of mobile media surveillance as emerging from dominant three kinds of surveillance. In addition to the traditional notion of surveillance, characterized by its non-transparency by an authority such as the government, three other kinds of surveillance have been identified in the literature: voluntary panopticon, lateral surveillance, and self-surveillance.

Voluntary panopticon refers to the voluntary submission to corporate surveillance or what Whitaker calls the "participatory panopticon" (1999). A voluntary or "participatory" panopticon differs from older systems of 
surveillance in that it is consensual. The voluntary panopticon is based on a consumer society where information technology allows for the decentered surveillance of consumptive behavior. Participatory panopticon is very similar to participatory surveillance in that people willingly participate in the monitoring of their own behavior because they derive benefit from it (Albrechtslund 2008; Poster 1990).

Lateral surveillance is the asymmetrical, nontransparent monitoring of citizens by one another (Andrejevic 2006). With the advent of the internet and interactive media, people have similar technological capabilities previously held exclusively by corporate and state entities. As such, citizens can monitor other citizens' behavior through nonreciprocal forms of watching. Everyday people can search for information about other citizens without their knowledge or permission. The advent of social media has given rise to other forms of lateral surveillance such as Marwick's (2012) aforementioned "social surveillance," which suggests a mutual surveillance among actors using social media. Like lateral surveillance, social surveillance involves nonhierarchical forms of monitoring (i.e., not involving the state or corporate entities) among everyday people. Unlike lateral surveillance, social surveillance suggests that people engage in permissible and reciprocal forms of watching.

The last kind of surveillance is self-surveillance. Meyrowitz defines self-surveillance as "the ways in which people record themselves (or invite others to do so) for potential replaying in other times and places" $(2007,1)$. Technologies such as video cameras and cameraphones allow people to capture aspects of their lives to replay later. The ability to record oneself can lead to the scrutiny of mundane behavior, which can fundamentally change one's understanding of that behavior or event. The recorded behavior has power over our lived lives.

An important aspect to understanding the mundane and intimate ways surveillance plays out is acknowledging that it is, as both concept and practice, informed by cultural context. Just as what constitutes participation and power is culturally specific, so too do these nuances need to be identified and appreciated. For example, in China, there are various forms of horizontal and vertical forms of surveillance that happen in and around familial practices. In a Chinese context there are three key notions that inform our definition一watching (看护, $\mathrm{Kan} \mathrm{Hu}$, which means keeping an eye on someone); overseeing (监看, Jian Kan, is to follow an activity or an entity to make sure that it operates normally and correctly) and surveillance (监控,Jian Kong, is used where power, authority, and rebellion are often involved). In particular, it is a combination of both Kan Hu and Jian Kan that play out and through the micro-coordination of care at a distance through mobile media practices. 
In Japan, 監視 (kanshi) refers to vertical surveillance whereby something or someone can prevent problems from happening. 観察 (Kansatsu) refers to watching which is viewed as more neutral. 監督 (Kantoku) signifies careful overseeing whereby someone like director or manager who is in charge of organization supervises his or her members. However, when it comes to family, 見守る (mimamoru) is more appropriate and often used by parents when they care for their children-it means to follow or to watch out for one's safety. This notion resonates with the earlier discussion of "careful surveillance" (Hjorth, Richardson and Balmford 2016).

These culturally different notions of watching, surveillance and care need to be taken into consideration. Western ideas about surveillance are not necessarily relevant in many cases where culturally specific notions of care are at play. In the next section we discuss some of the many ways friendly informal surveillance can manifest within familial care at a distance.

\section{Care at a Distance: Examples of Families and Friendly Surveillance}

\section{Melbourne}

Within family relationships, surveillance takes many forms and often involves different textures of care. In Melbourne we find 53-year-old Nancy and her daughter 30-year-old Jessica. Nancy discussed a type of surveillance with monitoring her daughter Jessica's Facebook activities, where she didn't actively monitor, but rather, being one of Jessica's contacts allowed her to be a constant benign presence. As Nancy explained:

I remember when my daughter asked me for Facebook, I said fine, but I need to be your friend. That's the condition and then I explained to her why, it's not that I want to check on you, it's that in case anything happens, I have the access and I know.

Nancy had never asked for Jessica's passwords and they had never discussed monitoring Jessica's whereabouts apart from discussion. In a discussion on tracking apps, Jessica said:

If it makes her (Nancy) feel better yeah, yeah. But I wouldn't be too happy about it. I mean, I've got nothing to hide, you just want your own privacy. And you don't want that kind of tension to be there. Or misunderstandings 
to occur. So, I'd probably like try to talk her out of it, but I guess if that's how she feels, yeah. I don't know how I'd feel about that.

Nancy and Jessica did not feel the need to talk about surveillance mechanisms specifically in relationship to devices as they had established clear expectations generally. From Nancy's perspective:

I suppose I give her that trust, we've discussed it before and I've said to her once that line is crossed once, that's it. If we can maintain that, that will be good on both sides ... thankfully so far, we have built up that trust. I think it's important that at the beginning you set the boundaries and it's easy to manage both sides where we stand. So she knows that she can go out once she gets that permission but I request that she informs me more so for safety and she's done that, so far, we had a good understanding.

Nancy and Jessica's situation illustrates how expectations are integral to maintaining a positive mother-daughter relationship. Nancy expressed several times what she could have done in terms of monitoring Jessica's activities, but she didn't. She constantly used Jessica's laptop and knew it was not password protected, but never checked Jessica's email if open. As we discuss in Chapter 7 , Mason $(1996,15)$ draws an important distinction between the ideas of caring about, which comprises the experience of the feeling and caring for, involving active care directed towards another person. Digital devices and social media in particular have played a role in navigating both expressions of emotion, particularly for transnational families.

Baldassar $(2007,391)$ explores the exchange of emotional and moral support between transnational families and she likens "staying in touch" to reciprocal gift exchange. Drawing on di Leonardo's $(1987,440)$ notion of kinwork-which recognizes the multiple efforts invested in maintaining familial relationships-Baldassar $(2007,394)$ explores types of care as routine, ritual and crisis and their consequences. The frequent contact and sense of co-presence afforded by social media also means that transnational families can be integrated into each other's lives at a distance. Yet, as Wilding (2006) observes, the capacity for increased communication can also create obligation, where transnational family members come to expect frequent and regular contact and for some, increased burden to visit.

Four of the participants from three different households explained how WhatsApp groups with family members and old friends overseas facilitated closer relationships, yet also caused different levels of intrusion throughout the day. For the middle aged and elderly participants, WhatsApp groups 
were seen as an extended form of socializing when they would otherwise remain at home throughout the week when not working, and for the younger participant, the constant "ping" of the WhatsApp group (which she chose to mute) was also a constant distraction.

Esther described how being in a WhatsApp group with her former classmates from primary and secondary school — who she had known for nearly 50 years - inspired her to organize trips with small groups of friends. She received photos and updates when members of the group in Malaysia met together. Throughout the period of research, Esther hosted visitors from two separate small groups of friends who visited Melbourne and even stayed with her and her family. In Esther's case, more frequent communication enhanced these relationships and she did not see those connections as a burden, in part because she was moving towards her retirement and felt she would have time to enjoy the connections.

The expression of care as self-expression but also as directed actions more broadly relates to expression of emotions as a more general mode of communication. A cultural approach to emotions argues that experiences of emotions are culturally embedded, that is, people from different cultures experience different emotions in different ways (Lutz and White 1986). Social media, as one example, constitutes a framework for the expression of emotions but also for the reciprocal recognition of the experiences of others.

As we explore in Chapter 8, withholding from posting certain content on Facebook avoids attracting unwanted attention but reflects an acknowledgement of how others feel about postings. Yet, the same participants who refrain from posting things that might be perceived as showing off still "like" the posts of others that they see as posts intended to "show off." Between family members, it was generally seen as more favorable to acknowledge the posts of others rather than to ignore or dismiss them, even though the person "liking" reserved some judgment about the post.

Yet, as much as the experience of emotions around devices might be influenced by factors such as cultural embeddedness or the state of relationships, devices are also influencing the cues in the expression and experience of emotions. Vincent and Fortunati $(2009,13)$ describe mobile emotions as "mediated emotion," that is, "emotion that is felt, narrated or shown, produced or consumed that is mediated by a computational electronic device." Lasén $(2004,1)$ describes mobile phones as having become affective technologies, or objects that mediate the expression, display, experience and communication of feelings and emotions.

Yet, as Lasén, Vincent and Fortunati also observe, as much as mobile media users have an emotional relationship with their devices and are 
attached to them, mobile media have become technologies that mean multiple forms of intimacy (Lasén 2004; Vincent 2005; Fortunati 2002). Smartphones as devices that converge technologies for communication, information and entertainment also means that the range of emotional experience with the phone is much wider. The following cases illustrate some of these complexities, where emotions were not only about connectedness with others and maintaining relationships, but were also experienced around addiction, attention, boredom, self-consciousness and triggering. If one form of surveillance within relationships with others is based on care, its adversary is self- surveillance which results in self-monitoring and self-judgment around one's usage of the phone and one's emotional attachment to it.

\section{Tokyo}

As noted earlier, Japan has various perceptions that related to different forms of horizontal and vertical, social and organizational forms of surveillance. In particular, it is 見守る (mimamoru) that is more appropriate to familial forms of surveillance and often used by parents when they care for their children.

In Tokyo, LINE was viewed as a way to keep a friendly, non-invasive co-present eye on family and friends. A key example was Haruko, a 22-yearold undergraduate student, who lived with her parents and used LINE as a form of mimamoru with her family. Her family consisted of her parents and younger brother who had been studying abroad for some years. When the big earthquake occurred in the northern part of Japan in 2011, Facebook was the only functioning communication line which every member of her family could access and communicate with each other, while phone, SMS, and email were not. Since then, Haruko had not used Facebook to connect with her family. From 2013, when all the family members started to use smartphones, communication with her family shifted to LINE.

Haruko made three groups on LINE for communicating with her family members: an "official" group of family, a group of family members who lived together (excluding her brother), and a group with her mother. In the official group of family, they told one another what was going on in their life. As Haruko notes:

For sharing information with family, Facebook is not convenient. LINE is very useful in sending messages, pictures, videos, etc. When our family members' lifestyles started to change, we became LINE users. LINE's 
design is very nice. It feels like we are talking. It's not like e-mail. When my brother graduated from a college abroad, he sent us a warm message and pictures on LINE for telling his graduation and appreciation to the family. At that time, I was also away from Japan for studying and my father was also on a business trip. All the family members were in different places. It was very moving to see my brother's message on LINE. I also sent them a message from abroad. It was very warm moment. I was really relieved.

The group of family members who lived together coordinated life and meals daily, but in the group with only her mother their practices differed. As Haruko described:

After my brother and I grew up, my parents spend more time together. I always worry about them if they are nice to each other. Because they are so different, they often fight. When they were traveling to Hokkaido, my father sent pictures with their smile to the official group of family. But my mother sent me a message to the group of us referring to feeling tired and bored. So, I sent a message for asking her if she is ok. I often send my mother stamps, too.

She never performed this kind of one to one communication with her father on LINE; instead she used the Facebook "check-in" function to communicate with her father. Haruko discussed the motivation for this practice as follows:

My father told me to check-in on Facebook at all the places that I go to when I travel. He gets angry if I don't. He said that I don't have to call him, he can make sure that I am safe when I check-in. That's why I check-in at least once a day at the hotel or at the station when I travel.

In contrast to Haruko's family, Maki and her mother Eriko primarily used LINE for familial sharing and co-present intimacy because "it's easy" to communicate. Eriko explained, "e-mails have to be opened one by one. But on LINE, we can smoothly go back to previous messages." Maki believed that communication between the two had increased since they started using LINE because "it's much easier to use LINE than to send e-mails from mobile phones." In this family of only a mother and daughter, friendly unidirectional surveillance in the form of mimamoru operated as a form of care.

Often Eriko and Maki only sent a word or two with photos and stamps to each other on LINE. The conversation was usually about everyday topics like “what time do you go home?" or "Shall I fix your skirt?" They used 


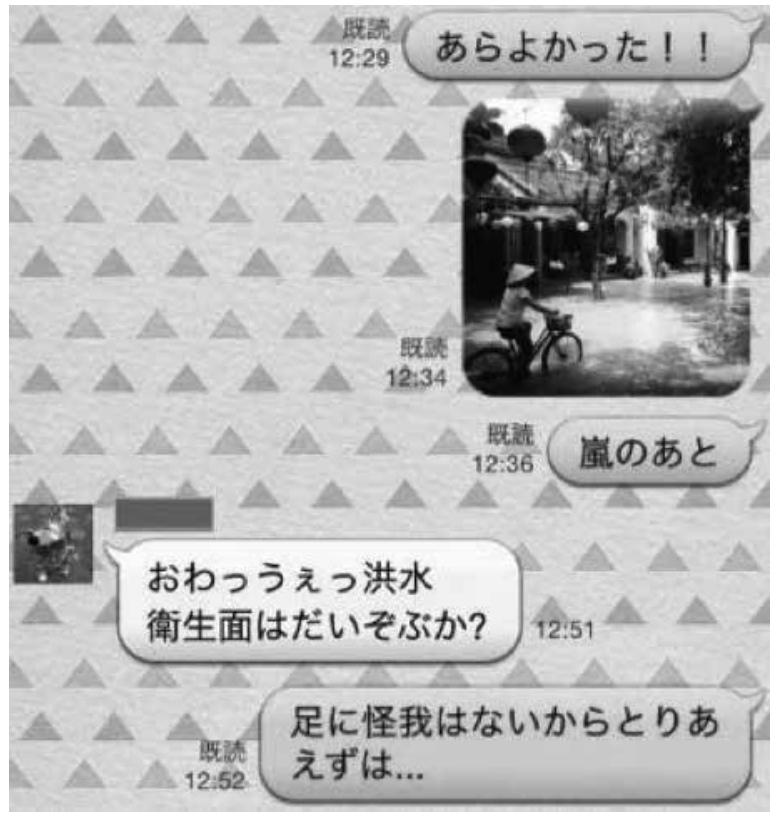

Figure 3.1: Maki and Eriko exchanged messages and photos on LINE for confirming the safety while travelling abroad

LINE not only for everyday communication but also for confirming the safety while traveling abroad. Sometimes when Eriko didn't notice Maki's messages, "Maki makes a single-ring-and-hang-up call. Then I notice that I got the red flag." A single-ring-and-hang-up call was a sign of "Check the message on LINE." While this had been the pattern in recent years, Maki would soon marry her boyfriend and leave her home. At the time of our last interview, they were not sure if and how communication between them might change in the future.

Our examples demonstrate how social surveillance is realized and practiced in Japanese families. One of the key incentives for mutual monitoring is the issue of safety. For example, within the context of daughter-father communication, sharing locational information by "checking in" was a way of developing care at a distance and maintaining relationships. Particularly, after the experiences of the severe earthquake in March 2011, Japanese family understandings and uses of social media changed, and mutual monitoring emerged as a basic condition through which peaceful relationships with family members are maintained.

It is also not uncommon to have multiple channels of social surveillance. For example, Haruko communicated with her parents through one LINE group whilst at the same time connected to her mother alone in another 
one. Interestingly, those two channels were active simultaneously. In each group Haruko and her mother were enacting different roles, shifting their mode of communication depending on the group to talk to.

\section{Shanghai}

In Shanghai there were informal and formal ways in which surveillance plays out in horizontal and vertical ways. Watching (看护, $\mathrm{Kan} \mathrm{Hu}$ ) means keeping an eye on someone, or paying attention to them. When children are little, parents watch them in case they fall down; when they are far away from home, parents may watch them by following their updates closely in WeChat Friends' Circle, or by seeing who they are interacting with in QZone (a website where QQ users can write microblogs and tweets). Overseeing (监看, Jian Kan) is to follow an activity or an entity to make sure that it operates normally and correctly. Parents ask about children's daily activities via instant messages like WeChat, where topics range from their academic performance to their employment. Parents oversee not only to have an idea of children's daily life, but also to ensure that their children are doing well.

Surveillance (监控, Jian Kong) is used in careful watching where power, authority, and rebellion are often involved. For example, some parents download software compulsorily to surveil their children's using of internet, like LvBa. These apps can block pornographic websites, limit children's using time and restrict online chatting. Surveillance is also used for situations where institutions conduct supervision and controlling behaviour-like the Great Firewall of China, which is integral to internet surveillance.

Due to the strong pervasion of mobile internet and rapid development of various location-based services, online "check-ins" have become a trend, especially among the young. Ai, a 24-year-old graduate student noted that, "I used to keep the habit of online check-in on Jiepang.com (the Chinese version of Foursquare). Not for special reasons, just find it interesting to be the 'landlord' (the one who has the most check-in record in certain area)." As Ai described in more detail:

There's one benefit of using Jiepang.com to check-in - that is, I do not have many friends on Jiepang.com, so I can have a secret room for myself. I can say anything I want there without concerning for my parents or others.

Ai was not the only interviewee who expressed a desire for privacy on digital media. As WeChat has gained increasing popularity in China, friendly surveillance emerged as the key expression of intergenerational relationships 
on WeChat. Due to the special 4-2-1 family structure in the wake of China's one-child policy, children have undoubtedly become the focus of the family. When children were old enough to move away for study, parents used social media to provide a constant care at a distance. Because WeChat also allows online check-in through Moments, parents now had a new way to learn about their children's safety and engage in online caring. However, such friendly surveillance could become a burden for some kids.

Whereas parents emphasized how they benefited from knowing about their children's immediate status and detailed daily lives via the WeChat, children held conflicting attitudes about adding parents as "friends" on social media, and on the value of locative sharing. On the one hand, the young people agreed that the locative social media and information sharing facilitated their communication and intimacy with their parents; on the other hand, the constant monitoring from the parents felt like "over-care." 25-year-old Chen was such a typical case. She described her mother as crazily concerned for her safety. Her mother tracked her "footprint" on WeChat. "My mom wants to know every motion of mine," Chen complained. Almost every time she updated new moments or pictures, her parents vigilantly asked details about them.

One time, Chen went to a friend's house and posted some photos on her WeChat. Her mother immediately asked her on their WeChat family chatting group, "Where did you go? Where is the place in the photos? Did you go there alone?" Chen was then obligated to respond to her parents with details. If she did not report her activities in time, her mother would keep asking her questions on WeChat, or might even call her.

It should be noticed that it is not the locative media, per se, that is the source of over-caring for Chen; her mother has engaged in this kind of "over-care" since Chen was a little girl. The difference was the media. In the past, her mother called her at night almost every day to check if she was staying at her dormitory, the "safe area." Chen remembered her mother even called the police when Chen got back to campus half an hour later than usual and her mobile phone was unfortunately powered-off. To some extent Chen had adjusted to this situation, "I have to report my 'doing list' to my parents if I don't use WeChat." The over-care online with WeChat is an extension of existing offline daily over-care from the parents.

23-year-old Ti, on the other hand, described herself as quite an independent girl. She lived in Beijing for four years before she began her Masters study in Shanghai, and had become used to managing her personal life by herself and didn't want to explain the details to her parents. As she explained, "my parents only need to know that I'm healthy and happy." Luckily in the past her parents had no channels to monitor her mobility and life trivia, but things 
started to change. When Ti uploaded a photo, her father often commented and asked, "Where are you? Where is it?" She felt bothered, "What's the use of that! They don't know much about my school things; they don't understand and can't help." Ti said that she would like to share things with her parents if she went somewhere interesting, but she didn't want to be interrogated.

Unlike Chen's mother, 28-year-old Ke's parents encouraged her to go out. So Ke did not care that her parents could see her location on locative social media, but she preferred not to share other things. There were times when Ke complained on social media and her parents read the post and asked her what happened. When she posted photos late at night, her mother chided her and went on about how this could be detrimental to her health. In these situations, Ke felt the over-care from her parents. To her, they were just "negative emotions that everybody can have" and "I don't want them to worry about that." For this reason, she blocked her parents a few times on WeChat. This case illustrates another common phenomenon caused by parents' friendly surveillance on digital media: "worry about worries." That is, the "over-care" from the parents makes their children recognize the worries of their parents, which conversely becomes the worries of the children.

\section{Cultural Understandings of Friendly Surveillance}

As this chapter has demonstrated, there is a need to understand friendly notions of surveillance in ways that are culturally specific. The entanglement of watching and friendly surveillance takes various textures across individual, social and organizational layers. These layers are specific to the cultural context and what Herzfeld (1997) calls "cultural intimacy." In China, for example, with high horizontal state and organization surveillance (and introduction of social credit), watching by parents of children takes on a different dimension.

Just as mobile media has diversified the horizontal and vertical ways in how surveillance plays out, it is also important to understand how culturally specific notions of watching, power and surveillance inform the practices. Careful surveillance is a significant part of maintaining Digital Kinship. It is about the affective labor of doing intimacy and its attendant boundary work.

In this practice of careful surveillance, Playful Kinship is crucial. In the next chapter we move into the second section of the book-Playful Kinship. Here we argue, in keeping with Sicart (2014), that the playful is a key mode for digital media engagement. From paralinguistics to camera phone images, play operates as the logic for modes of sharing. 


\section{References}

Albrechtslund, Anders. 2008. "Online social networking as participatory surveillance." First Monday 13 (3): article 6. http://firstmonday.org/article/ view/2142/1949/. Accessed 20 May 2016.

Andrejevic, Mark. 20o6. "The discipline of watching: Detection, risk, and lateral surveillance." Critical Studies in Media Communication 23: 391-407. DOI: 10.1080/07393180601046147.

Baldassar, Loretta. 2007. "Transnational families and the provision of moral and emotional support: The relationship between truth and distance." Identities: Global Studies in Culture and Power 14 (4):385-409. DOI: 10.1080/10702890701578423.

Clark, Lyn Schofield. 2012. The Parent App: Understanding Families in the Digital Age. Oxford: Oxford University Press.

di Leonardo, Micaela. 1987. "The Female World of Cards and Holidays: Women, Families, and the Work of Kinship." Signs 12 (3): 440-453.

Ellison, Nicole B., Charles Steinfield and Cliff Lampe. 2006. "The benefits of Facebook 'Friends': Social capital and college students' use of online social network sites." Journal of CMC 12 (Issue 4 July): 1143-1168. DOI: 10.1111/j.1083-6101.2007.00367.x.

Fortunati, Leopoldina. 2002. "The mobile phone: Towards new categories and social relations." Information, Communication and Society Journal 5 (4):513-524. DOI: $10.1080 / 13691180208538803$.

Herzfeld, Michael. 1997. Cultural Intimacy. London: Routledge.

Hjorth, Larissa, Ingrid Richardson, and William Balmford. 2016. "Careful surveillance and pet wearables: At home with animals." The Conversation, Sept $5^{\text {th }}$, https://theconversation.com/careful--surveillance--and--pet--wearables--at-home--with--animals-63883. Accessed 20 June 2016.

Humphreys, Lee. 2013. "Mobile social networks and surveillance: Users' perspectives." Media, surveillance, and identity: A social perspective, edited by André Jansson and Miyase Christensen. New York, NY: Peter Lang.

Joinson, Adam. 2008. "Looking at, Looking up or Keeping up with People? Motives and Uses of Facebook." CHI 2008 Proceedings · Online Social Networks April 5-10. Florence, Italy. http://digitalintelligencetoday.com/downloads/ Joinson_Facebook.pdf. Accessed 20 May 2015.

Lasén, Amparo. 2004. "Affective technologies: Emotions and mobile phones." Receiver 11. www.receiver.vodafone.com. Accessed 20 May 2010.

Lutz, Catherine, and Geoffrey M White. 1986. "The anthropology of emotions." Annual Review of Anthropology 15: 405-436.

Marwick, Alice E. 2012. "The public domain: Social surveillance in everyday life." Surveillance and Society 9 (4): 378-393. 
Mason, Jennifer. 1996. "Gender, care and sensibility in family and kin relationships." Sex, Sensibility and the Gendered Body, edited by Lisa Adkins, and Janet Holland. 15-36. London: Palgrave Macmillan.

Meyrowitz, Joshua. 2007. "Watching us being watched: State, corporate, and citizen surveillance." Paper presented at symposium, The End of Television? Its Impact on the World (So Far), Annenberg School for Communication, University of Pennsylvania, Philadelphia, PA.

Nippert-eng, Christena. 2010. Islands of Privacy. Chicago: Chicago University Press.

Poster, Mark. 1990. The mode of information: Poststructuralism and social construct. Chicago: University of Chicago Press.

Sengupta, Somini. 2012. “'Big Brother'? No, It's Parents." New York Times, June 25, http://tinyurl.com/aysm5ud. Accessed 1 April 2016.

Tokunaga, Robert. 2011. "Social networking site or social surveillance site? Understanding the use of interpersonal electronic surveillance in romantic relationships." Journal Computers in Human Behavior 27 (2): 705-713.

Vincent, Jane. 2005. "Emotional attachment and mobile phones." Thumb Culture: The Meaning of Mobile Phones for Society, edited by Stefan Bertschi and Peter Glotz, 117-122. Berlin: Transcript Verlag/Transaction.

Vincent, Jane, and Leopoldina Fortunati. 2009. Electronic emotion: the mediation of emotion via information and communication technologies. Interdisciplinary Communication Studies 3. Oxford UK: Peter Lang.

Whitaker, Reg. 1999. The end of privacy: How total surveillance is becoming a reality. New York: The New Press.

Wilding, Raelene. 2006. "Virtual' intimacies? Families communicating across transnational contexts." Global Networks 6 (2): 125-142. DOI: 10.1111/j.14710374.2006.00137.x. 

Section II

Playful Kinship 



\title{
4. Digital Gifts and Rituals
}

\begin{abstract}
In Chapter 4 we revisit historical discussions around mobile media as gift and the important anthropological meanings of the gift as a practice into power relations and rituals, to think about how we might expand upon this practice in terms of location. Here location, co-location and co-presence are revisited.
\end{abstract}

Keywords: gift-giving; co-presence; location

In Tokyo, Shizuka, a 21-year-old undergraduate student, lived with her mother (50-year-old), father (54-year-old) and younger sister (19-year-old). She used LINE regularly with her family. Her father worked in a major consumer electronics company and knew a great deal about the latest equipment such as computers and smartphones and was also an early adopter of LINE (See Chapter 2). Shizuka's mother had also started to use smartphones and LINE a few years earlier. Initially, she was not very comfortable using social mobile media, but over the past year her husband and her daughter had used stamps to integrate her into family life.

For Shizuka's family, stamps on LINE became one of the daily rituals of family life. Building on the use of emojis and kawaii culture in Japan, stamps provided playful ways to share feelings and emotions, providing a sense of co-presence throughout the day. Often the family would share stamps during specific moments of the day such as lunchtime and at the end of the day.

LINE had even invented stamps to share just between parents-conveying tacit and phatic forms of communication. Emojis and their next iteration, stamps, amplify the significant symbolic role of mobile media in maintaining co-present feelings and rituals when physically distant. They play into broader gift giving cultures that are both culturally specific and also transnational.

Hjorth, L., K. Ohashi, J. Sinanan, H. Horst, S. Pink, F. Kato, B. Zhou, Digital Media Practices in Households: Kinship through Data. Amsterdam: Amsterdam University Press, 2020 DOI 10.5117/9789462989504_CHO4 


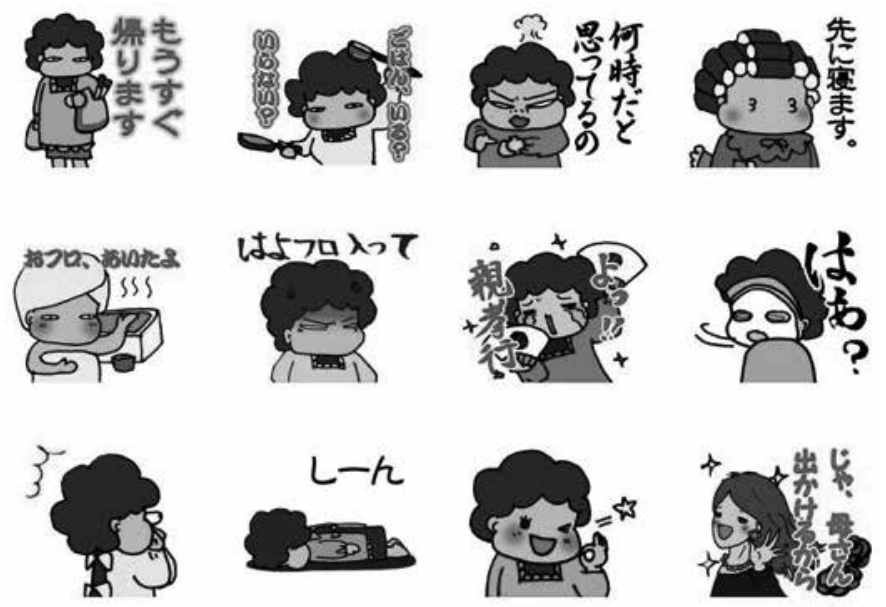

Figure 4.1: LINE stamps for mums

Building upon the discussion of friendly surveillance in the previous chapter, this chapter highlights the more playful forms of kinship-including the exchange of gifts and daily rituals - that are emerging through locative media. Gift cultures have a long and culturally specific history. The symbolic role of gift giving - as a practice of reciprocity, obligation and negotiating power relations - has long been attached to mobile media cultures (Taylor and Harper 2002). Gift exchange and attendant rituals help to make visible issues such as obligation and care and the multiple ways that locative and social media has come to shape digital kinship. Specifically, this chapter highlights how everyday play with digital gifts and rituals in contemporary family life enables the maintenance of family through creating forms of co-presence and related feelings of intimacy.

\section{The Cultural Dimensions of Gifts and Rituals}

There is nothing inherent in objects that designate them as gifts; objects can almost always follow varying trajectories. Precisely, gifts are not objects, but transactions and social relations (Frow 1997, 124).

Within disciplines such as anthropology, gifts and rituals represent some of the glue that keeps society cohesive. Whether it be through non-capitalist forms of exchange such as the Melanesian kula ring-a complex system that govern the rules of exchange and reciprocity (Weiner 1992; Malinowski 2014; Mauss 2016) — or the Native American potlach where gift giving is tied 
to forms of wealth display (Mauss 2016). There are also the more mundane practices around the sharing of food among families in the US over thanksgiving (see Kohn 2013) or gift giving at holidays such as Christmas (Miller 1995). In consumer cultures such as the US, gifts play an important role in the sustainability of the broader social structure.

Whether or not the rules of society include more calculated forms of reciprocity or generalized reciprocity for the broader social group remains a key issue. For example, in Malinowski's Argonauts of the Western Pacific (2014), he argues that reciprocity emerges as a key theme, but not in terms of mutual benefit. Rather, reciprocity is seen as an uneven power relationship that favors the giver of the gift, forcing the recipient into an ongoing "chain of reciprocal gifts and counter-gifts" (Ibid., 40) in order not to "lose face" within the tribe (see Weiner 1992 for a discussion of the gendered dimensions of gift giving).

Comparing different forms of gift exchange around the world, Mauss departs from Malinowski by reviewing the notion of reciprocity as a process primarily derived from (and also perpetuated by) underlying societal rules (Bergquist and Ljungberg 2001, 308-309; Taylor and Harper 2005, 441). For Mauss, it is impossible to extricate gift exchange from the social because gift exchange is itself the basis for all forms of exchange within many nonWestern societies, "a total social fact" that underpins all forms of social exchange be they religious, social, moral, juridical, familial or economic (Bergquist and Ljundberg 2001, 309; Mauss 2002, 3-4; Jarrett 2015, 206-207, 211).

Importantly, Mauss also refines Malinowki's ideas on reciprocity by aligning reciprocity with obligation. In doing so, Mauss identifies three types of obligation relative to gift exchanges - the obligation to give (one must give), the obligation to receive (one must accept the offering) and the obligation to return/reciprocate (when given a gift, an appropriate gift must be returned) (Frow 1997, 108; Mauss 2016, 10, 50). This notion of social obligation is crucial—often shaping contemporary motivations and practices around the use of mobile media for care at a distance. It is important to recognize that care isn't always without obligation. For example, parents and children share particular rituals around digital kinship that are underscored by obligation (i.e. obliged to respond to a social media post).

John Frow in Time and Commodity Culture (1997) — and "Information as Gift and Commodity" (1996) — addresses an alternative aporia: the disparity when gift and commodity economies coincide. For Frow $(1997,102,124-126$, 130), there are no societies in which gift and commodity exchanges are not co-present-nor can the two modes of exchange be seen in purely binary 
term. While gifting and commodities can be understood in "ideal" terms as distinct systems, whenever they meet a "recurrent lack of commensurability between a gift relation on one side and a commodity relation on the other" emerges (Frow 1996, 97-98). Ultimately, Frow concludes that while reading information commodification through the lens of gift/commodity economies is useful, "the concept of gift is irrelevant to the structural understanding of modern societies, with the exception of the micro-level of everyday life" (Ibid., 107-108).

Turning to the mobile phone, Alex S. Taylor and Richard Harper's (2002) "Age-old Practices in the New World-and "Gift of the Gab" with Sara Berg-picks up directly from Frow by analyzing social practices of gift exchange through "everyday" observation. Their chapter-a four-month ethnographic study of British teenagers' use of their mobile phones-shows that the teenagers' mobile practices closely resemble notions of gift exchange (Berg, Taylor and Harper 2005, 440). Specifically, Mauss' system of reciprocity was particularly prevalent wherever SMS transactions between teens was observed (Taylor and Harper 2005, 441).

From feeling of obliged to respond to SMS transactions through to carefully managed intervals between receipt of SMS and reciprocal response, the "social obligation to exchange" was displayed by all participants variously throughout the study (Ibid., 440-444). A key feature of the study was the mixture of reports based on instances where participants were engaging in mobile communications in isolation and also using their phones in the company of others. In either case, patterns of behavior akin to Mauss' system of reciprocity generally exhibited, especially in relation to social status based on open displays of friendship/alliance or rivalry-passing phones to one another, establishing "sideplays" by only sharing with one other group member, etc. (Ibid.).

What becomes apparent in our discussion of the gift—as part of kinships rituals - is the way in which media practices can function to both extend existing rituals of reciprocity and exchange as well as creating new ones. By viewing the context of Australia, which shares many gift giving rituals with other Western contexts such as England and the US, alongside two locations with rich histories of gift giving - Japan and China-we can see the multiple ways in which the gift as kinship plays out. For example, Chinese notions of guanxi (social capital) play into gift cultures and power relationships (Hjorth and Arnold 2013).

Japanese culture traditionally values seasonal greetings and gifts in order to maintain relationships. In mid-summer and the end of the year, family, friends and co-workers send gifts to people such as colleagues, 
bosses, relatives, and teachers in order to express gratitude. It contributes not only to shaping and reshaping relationships, but also to acknowledge and feel the changes of season. By sending such gifts periodically, they nourish the sense of closeness or of growing together. Also, every January, they send postcards to friends, relatives and colleagues to greet the New Year (like the western tradition of sending/receiving Christmas cards in December).

While the Japanese custom of sending New Year's greeting still remains, in contemporary culture paper postcards are replaced by electronic versions. In particular, the active use of emoji or stamps over social media may resonate well with Japanese culture of periodical gift giving. As we highlight in this and the following chapters, sending photos and stamps can be understood as a form of gift, through which we develop and nurture our relationships and, through these practices, a remediation of postcards in the form of electronic paralinguistic sharing (Hjorth 2005).

In Shanghai, gift giving is one of the most important forms of social exchange. Gifts build up the inter-dependent relationship, i.e., Guanxi, between different people (see Yang 1994). Chinese society is rather mobile and individual-centered, and gifts play a vital role in maintaining, building and transformation interpersonal relationship in China (Jacobs 1979; Walder 1986; Yang 1989). According to Yoon et al. (2011), there are three primary goals to engage in gift giving practices in China: to manage one's social status, to maintain or improve relationships, as well as to demonstrate conformity to agreed social norms. Gift giving culture can be dated back to ancient China. As a Chinese saying goes, courtesy requires a return of visits received. A state of ceremonies as it is, China has numerous historical stories of gift giving and emphasizes the importance of two-way exchange of gifts both in the aspect of folk tradition and diplomacy.

During the Spring and Autumn Period (770 B.C-221 B.C), when the territory was separated into several states, there were gift-exchanging cases between regimes. It was also the Chinese tradition to send gifts among civilians. The most common and in-style way of gift giving today is the Red Pocket. Sending red pocket is a typical Chinese tradition, which means the seniors give lucky money to the young, in order to wish the young luck in the coming year. During the 2015 Spring Festival, WeChat developed its Red Pocket function into an app. According to Tencent (2016), the total sending and receiving amount of WeChat Red Pocket on the eve of Chinese lunar year reached 101 million.

In the following section we focus upon forms of digital gifts and rituals that have emerged in the intergenerational families in this study. Specifically, 


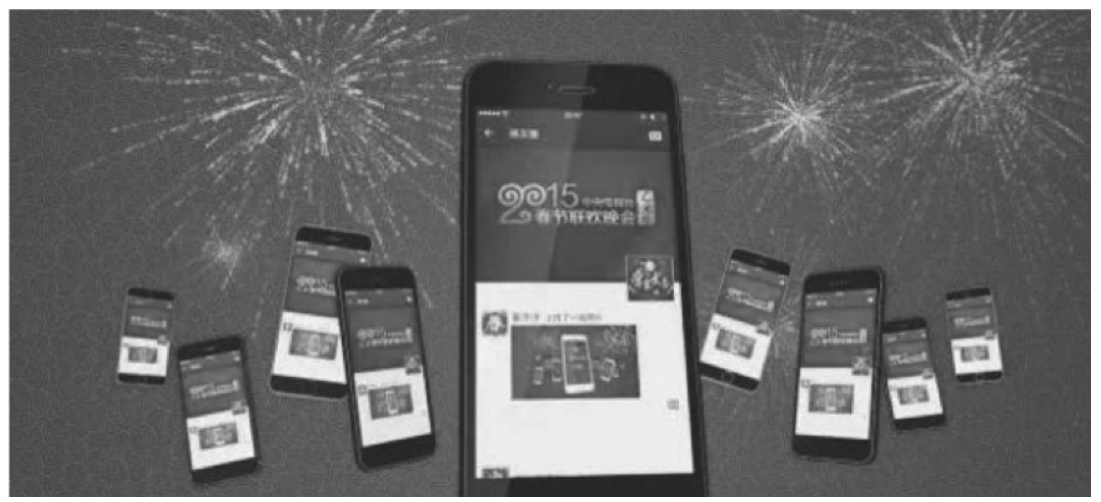

Figure 4.2: WeChat Happy New Year gifting

we will focus upon two, interrelated forms of gift giving which has become part of the playful ways in which kinship is produced and reproduced in Shanghai, Tokyo and Melbourne. The first is the use of digital gifts for mundane forms of domestic care where the intimate mundane emerges in and through social and locative media use. The second is the use of digital gifts to create and maintain intimate rituals within families and in the final section we see how practices around disclosure enable family members to enact one of the classic practices of gifting, keeping while giving (Raheja 1988; Weiner 1992).

\section{Digital Gifts and Domestic Care}

In Melbourne the gift plays a role in maintaining social relationships (Ling and Yttri 2001; Hjorth 2005). Texting and/or sharing location on the way home from work (e.g. "I'm on my way home, should I pick up milk"), is a mundane practice that reinforces ties of connection and the reproduction of the household, what Ling and Yttri have referred to as "micro-coordination" of family life. Here domestic care operated as a gift culture in the maintenance of daily rituals.

For 6o-year-olds Dina and Mike Harrison, texting at the beginning and end of Mike's day was a comfort. Dina retired in February 2015 and was adjusting to life at home in Melbourne. She also suffered from arthritis and didn't leave the house for prolonged hours. Mike worked in a state correction center, where his job could be dangerous at times. As he couldn't have his mobile phone on him, he would call Dina from a landline when he arrived and before he left for home. Mike explained that the end of the day phone 
call was to let Dina know his day at work had gone well with no incidents and also to find out what kind of day she had had, how she was feeling and if anything had been particularly difficult for her, in order to prepare himself for the evening. As Mike explained:

I can find out what kind of mood Dina is in, with her arthritis if she's having an ok day or a terrible day I'll know, sometimes I'll wish I could go home now but it does help me prepare for what I'm going home to. It is a mental thing, but you do it automatically. You can tell by the sound of her voice. But I also like to ring and say hi.

Mike also let Dina know if he was going to pass the local shops when he was on the way home, in case she would like anything, especially if she had not left the house during the day. Texting on the way home from work emerged as a consistent practice between couples at different life stages in Melbourne. Ben (31-year-old) and Jasmine, for example, sent text messages over Facebook Messenger almost every day in order to coordinate their evening. Ben played soccer a couple of days a week and Jasmine had work or social plans most evenings, so they often let each other know of their upcoming movements for the evening to coordinate having meals together, or so Ben could make plans if Jasmine was already planning to be out for the evening.

Another couple, Lily and Christopher Turner (30-year-old), had two sons aged three and five. Lily finished work half an hour before Christopher and arrived home to start making dinner. A usual evening involved Lily cooking after work, Christopher arriving home, where they would all eat together before giving the boys a shower, then half an hour of play or reading time before putting the boys to bed. Lily and Christopher also texted each other every day when they were leaving work. As Christopher described:

In terms of being considerate and knowing what to expect, it probably matters more with the boys. Like being home by 6 wasn't as big a consideration when you're not going to have dinner 'til 7 and it's not boys finishing dinner and getting ready for showers and things. 5 or 10 makes a difference with kids, it's not the be all and end all but it's helpful.

Lily added, "Or if they're giving me a really hard time. Sometimes I just need him home. Like if I know I just have to survive another 10 minutes and he'll be home." Like the use of phone calls to micro-coordinate daily life, gift giving in Japan can also be seen through the sharing of familial information regarding their day-to-day routines. 


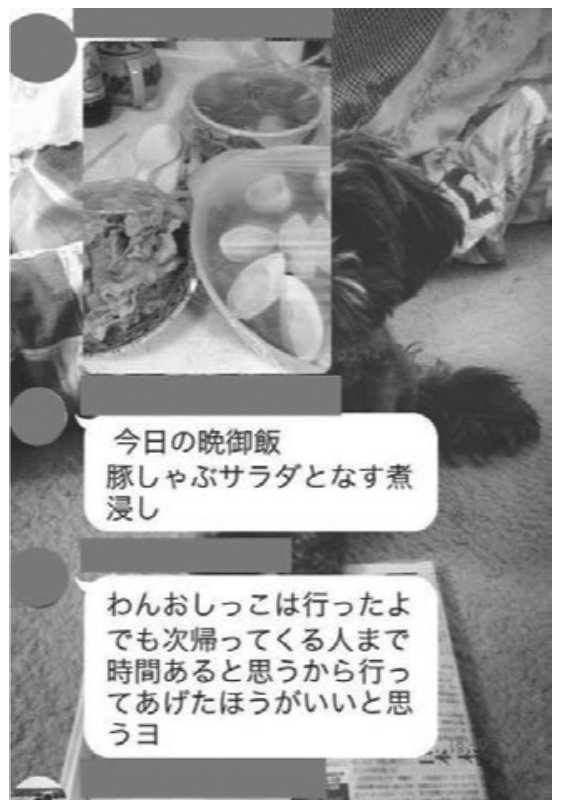

Figure 4.3: Saori and symbolic gift giving through maintenance on LINE

Saori, a 21-year-old undergraduate student, lived with her mother (49-yearold), father (51-year-old) and younger sister (21-year-old). Saori and her family used LINE for making contact with each other. In their LINE family group, the main topics were "food and the dog." Her mother would send a message about and a photo of "today's dinner" almost every weekday, usually to say something like, "please warm this before eating." They would also talk about who would take a dog for a walk.

Saori's mother's message "please warm this before eating" suggested that whereas family members were connected through LINE group to coordinate things, they would not be not eating together that particular day and the first person to get home might eat alone. In Shanghai, sharing location is also a normative practice between family members. For near-by family members, sharing locative information was a helpful practice for the micro-coordination of domestic life.

50-year-old father Jun and 45-year-old mother Qin exchange often through the family WeChat group. As a typical Shanghai father, Qin was in charge of preparing food for the family every day. In the red rectangle, Qin was saying that he was at the market and asking his family members for suggestions for the dinner. "It is really hard for me to design the daily menu," he said, "by asking my family's preference for dinner, it saved time." 
In essence, families in Melbourne, Shanghai and Tokyo used mobile, social and locative media to manage the vagaries of everyday life and the reproduction of the family. Whether it involved phone calls home to assess the mood of a spouse coping with an illness, a new life stage or the management of children or posts to share the preparation of dinner, social, mobile and locative media become part of the mundane forms of care in families. These small "check-ins" to micro-coordinate the activities of the household and the family thus represent forms of everyday (digital) gift giving in families. While mundane, family members noted their absence or delay, and the exchange of information was part of the reciprocity involved in reproducing the household.

\section{Digital Gifts as Intimate Co-presence}

Alongside the more instrumental use of the phone to micro-coordinate domestic life across the three sites in this study, we also saw the emergence of digital gifts in the full-time intimate communities (Ito, Matsuda and Okabe 2005) that constitute co-presence. Co-presence is an important part of maintaining social relations in everyday rituals. In our research, we saw the sharing of events happening in other places on a variety of platforms through tagging on sites such as Facebook, SMS direct messaging or WhatsApp. These often produced a sense of mundane intimacy, both creating and reinforcing a sense of familiarity.

In Shanghai, the creation of co-presence through social and locative media has become a key way through which families manage separation and distance. As children grow older, it is common for Chinese children to gradually leave their beloved hometown and start their new life alone in a faraway city without parents' company. Due to the one-child policy carried out in the 1980 s in China, the majority of Chinese university students today are the only children in their family. Parenting thus involved extensive care during their childhood and, as they move forward in life without their parents physically by their side, it also involves worry and concern. Sharing information and events within digital family groups thus effectively creates a virtual domestic space where family members feel co-present.

Thanks to the rich media content available on WeChat, Chinese users have various ways of sharing interesting life events with distant families so as to let remote family members get involved in the event at the same time by creating virtual co-presence. 


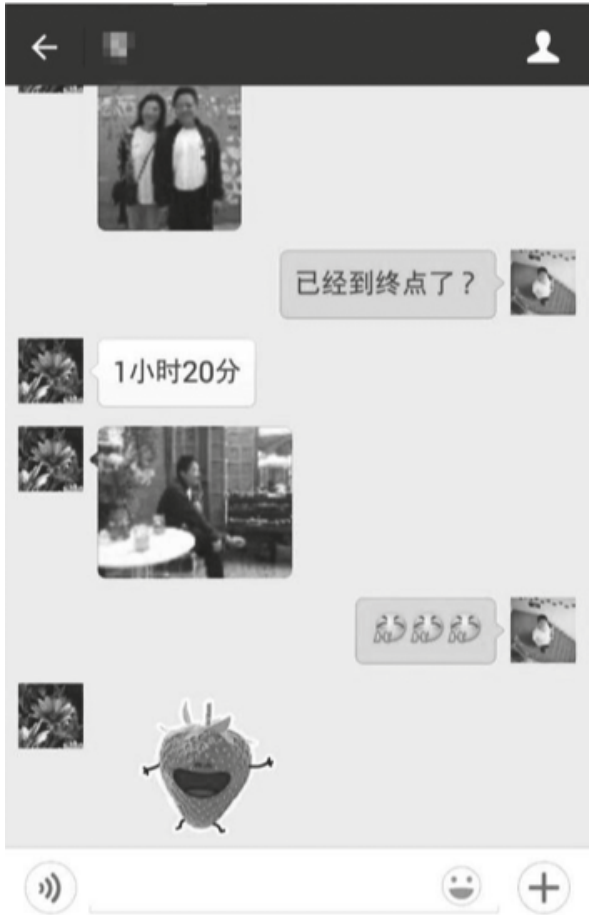

Figure 4.4: Kevin's family WeChat group

Figure 4.4 is a screenshot of Kevin's family WeChat group. Kevin, the participant on the right, was a 22-year-old boy from a Shanghai suburb who was studying in downtown Shanghai. Although Kevin was lucky enough not to leave his hometown to further his study, he only went back home once a month due to school commitments. According to his 50-year-old parents, they did not have much time to have face-to-face communication with Kevin. Therefore, the family WeChat group played a vital role in daily communication.

One weekend when Kevin was at school as usual, his parents took part in a hiking competition and took 80 minutes to finish the full distance. In Figure 4.4, Kevin's mother (on the left) shared a photo of both parents taken at the destination of the hiking competition and Kevin replied with cheerful emotional icons to congratulate. By sharing photos and text information, Kevin's family were able to be together during the hiking competition, which created a sense of daily intimacy, as if they were still by each other's side.

In Melbourne many of the migrant families we interviewed discussed sharing pictures of food, flowers or other things that they believed other family members would find of interest while separated. For example, Nancy 
used her iPhone to take, store and send photos and said, "I love photos. Sometimes, say, I take photos of my daughter, she is learning to cook so I take photos just to have a record." She sent several of her photos to her relatives overseas. As Nancy described:

My extended family, my cousins and aunties in Singapore. Just share because they don't have the opportunity to cook much over there. They do cook different things, they would like to bake more but they don't have that kind of equipment like here and it's hot over there as well so sometimes baking is not a conducive thing. And sometimes if we go for occasions we'll share, so some things that the family do.

While Nancy might share some of the things she did with her family on Facebook, where her photos might be seen by others, she shared photos of things she was more interested in with only certain people over WhatsApp such as photos of her garden and collages that she liked to do. She took photos and sent them overseas, commenting "because in Singapore they don't have much land to grow things so I love gardening, so I take all kinds of (photos) like plants and things like that and I show them fruits that's fruiting."

Through photos Nancy shared the kinds of things she would have shared with her extended family had she been living in Singapore. Occasions and meals were an important part of shared family time, where photos helped to fill some of the distance between relatives overseas, with WhatsApp being the key platform for the sharing of digital gifts.

\section{Keeping While Giving}

As noted in Chapter 3 , one of the more interesting digital gifts that appeared across the three sites was the increasing acknowledgement of the sharing of information as a gift. A key example is Satomi, a 31-year-old full-time housewife who lived in a town an hour and a half from the center of Tokyo by train. She lived in a duplex house with her daughter, her husband — who ran a company_-and his parents.

Satomi used LINE on a daily basis with her husband, his parents, and his sister, who were all registered as a group named "family." She frequently received messages from her husband, his mother, and his sister with messages such as "I've finished work now," "I got hung up now because of airplane's delay," or "Why don't you all have dinner at our place?" However, Satomi said, "I communicate with them only in a businesslike manner because I 

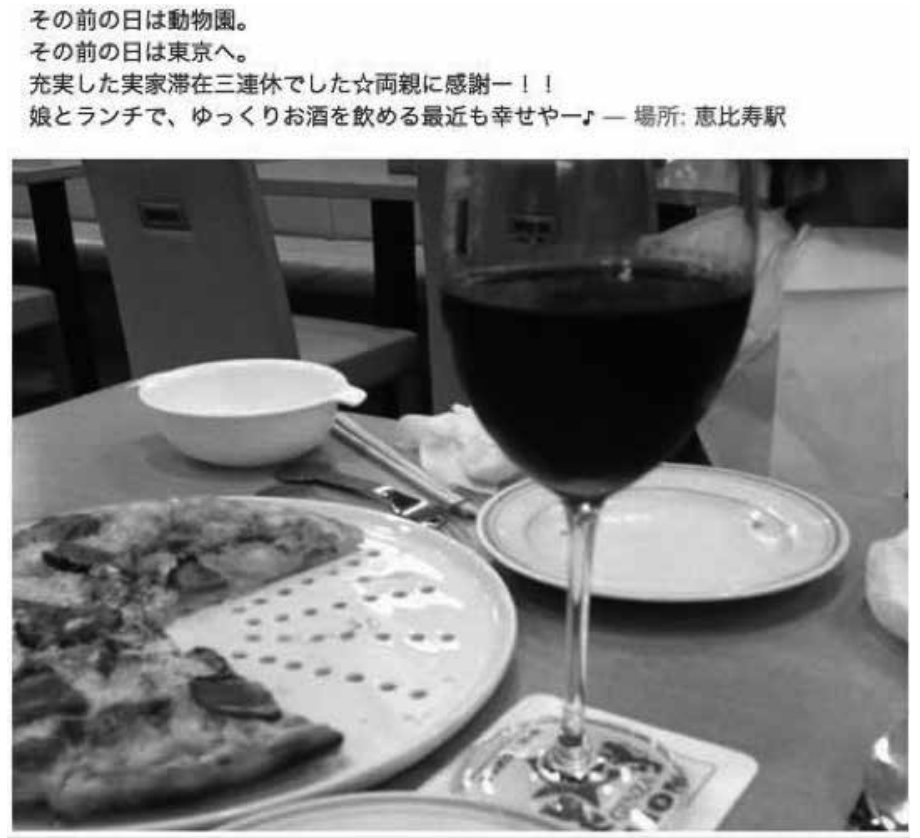

Figure 4.5: Satomi and co-presence as gift

never want them (husband's family side) to know what I did." She sometimes uploaded pictures to Facebook with location information, but not in real time.

In Figure 4.5 Satomi summarized her three-day holiday, together with a photo. She intentionally (or tactically) condensed information about her past three days into a single item in order to share vaguely with "friends" on Facebook. While Satomi wished to diarize her day-to-day activities on Facebook, she also attempted to keep a "proper" distance between her friends and family members by delaying her uploads or by blurring information. Thus, Satomi used social media to help construct distances and proximities, using it to keep her parents informed while also creating a "proper" distance. As highlighted in the extensive literature on gifting (see Weiner 1992), here we see that digital gifting can be used to keep distances as well as create intimacies.

\section{Conclusion: Gifts of Presence/Presents}

In this chapter we have focused upon the ways in which mobile, social and locative media have become part of the gift giving cultures in families across Melbourne, Shanghai and Tokyo. Building upon the extensive literature 
on gifting and the importance of gifting for maintaining social cohesion, we have highlighted the mundane practices of gifting that are occurring through digital means, and how these may be extending previous practices of sharing in families and cultures broadly. In the first section on care and reproduction, we looked at the ways in which WeChat and other platforms are being used to micro-coordinate family life, ranging from the planning of dinner to regulating the emotional state of partners.

The second section focused upon the use of digital gifts such as photos and other information to create a sense of intimacy and co-presence, particularly for families managing distance. In the third and final section we explored how one woman in Japan, Satomi, enacts a classic principle in gifting - the act of keeping while giving. While disclosing information and sharing images of travel and other activities, we saw how Satomi also attempted to maintain distance and keep control over the information that she shared.

As we demonstrated throughout the chapter, locative information conveys more precise information of the whereabouts of family members. It makes it easier for daily communication, which brings convenience for daily life micro-coordination within families. It also creates a sense of co-presence. Meanwhile, differentiated exposure of locative information on digital media indicates a sense of intimacy between certain members. Under such circumstances, sharing location becomes part of the gift economy within families.

\section{References}

Berg, Sara, Alex S. Taylor, and Richard Harper. 2005. "Gift of the gab." Inside Text: Social, Cultural and Design Perspectives on SMS, edited by Richard Harper, Leysia Palen, Alex S. Taylor, 271-285. The Netherlands: Springer-Verlag.

Bergquist, Magnus, and Jan Ljungberg. 2001. "The power of gifts." Information Systems Journal 11: 305-320. Accessed 10/20/2017. DOI: 10.1046/j.1365-2575.2001.00111.x.

Frow, John. 1996. "Information as gift and commodity." New Left Review 219: 89-108. _.1997. Tie and commodity culture: Essays on cultural theory and postmodernity. Oxford: Clarendon Press.

Hjorth, Larissa. 2005. "Odours of Mobility: Mobile Phones and Japanese Cute Culture in the Asia Pacific.” Journal of Intercultural Studies 26 (1- 2): 39-55. DOI: $0.1080 / 075^{256860500074003 . ~}$

Hjorth, Larissa, and Michael Arnold. 2013.Online@Asia-Pacific. London: Routledge. Ito, Mizuko, Misa Matsuda, and Daisuke Okabe (eds.). 2005. Personal, portable, pedestrian: Mobile phones in Japanese Life. Cambridge: The MIT Press. 
Jacobs, Bruce. 1979. "A preliminary model of particularistic ties in Chinese political

alliances: Kan-ch'ing and Kuan-his in a Rural Taiwanese Township." China Quarterly 78: 237-273. DOI: 10.1017/So305741000040467.

Jarrett, Kylie. 2015. Feminism, Labour and Digital Media: The Digital Housewife. London: Routledge.

Kohn, Tamara. 2013. "Stuffed turkey and pumpkin pie: In, through and out of American contexts." Cultural Studies 19 (1):50-72. DOI: 10.5130/csr.v19i1.3075. Ling, Richard and Birgitte Yttri. 2001. "Hyper-coordination." In Perpetual contact: Mobile communication, private talk, public performance, edited by James Katz. 139-169. Cambridge: Cambridge University Press.

Malinowski, Bronislaw. 2014. Argonauts of the Western Pacific. Translated by Adam Kuper. Revised edition. London; New York: Routledge.

Mauss, Marcel. 2002. Essai sur le don. First edition. Abingdon: Psychology Press. -2016. The gift. Translated by Jane I. Guyer. Chicago: HAU.

Miller, Daniel, Ed. 1995. Unwrapping Christmas. Oxford: Oxford University Press. Raheja, Gloria G. 1988. The Poison in the Gift. Chicago: Chicago University Press. Taylor, Alex S., and Richard Harper. 2002. "Age-old practices in the 'New World': A study of gift-giving between teenage mobile phone users." Proceedings of the SIGCHI Conference on Human Factors in Computing Systems. 439-446. New York: ACM. DOI: 10.1145/503376.503455.

Tencent. 2016. “2016 Annual Report.” http://www.tencent.com/zh-cn/content/ir/ rp/2016/attachments/201601.pdf. (In Chinese). Accessed 3 February 2019.

Walder, Andrew. 1986. Communist neo-traditionalism: Work and authority in Chinese industry. Berkeley: University of California Press.

Weiner, Annette B. 1992. Inalienable possessions: The paradox of keeping-while giving. Berkeley: University of California Press.

Yang, Mayfair Mei-hui. 1989. "The gift economy and state power in China." Comparative Studies in Society and History 31 (1): 25-54. DOI: 10.1017/So010417500015656.

-1994. Gifts, favors, and banquets: The art of social relationships in China. New York: Cornell University Press.

Yoon, Song, O., Kwanho Suk, Seon M. Lee, and Eun Y. Park. 2011. “To seek variety or uniformity: the role of culture in consumers' choice in a group setting." Marketing Letters 22: 49-64. DOI: 10.1007/s11002-010-9102-5. 


\title{
5. Playful Haptics in Families
}

\begin{abstract}
In Chapter 5 we begin to focus upon the ways in which families "play" with co-presence through different engagements with digital media. We discuss in detail our methods around understanding co-present practices, especially through "tactile digital ethnography." This chapter examines how studying the hand gestures in, and around, the screen might help us contextualize a more embodied practice of mobile media in the everyday.
\end{abstract}

Keywords: play; families; ethnography; tactile; methods

For 6o-year-old Patricia Williams in Melbourne, locative media like Google Maps felt counter-intuitive when understanding place. The recent retiree found digital maps disorientating. She preferred to trace the paper map with her hand-as if the haptics (touch) of the hand could enact the movement of her legs. She found the pulling, pitching and zooming in and out confusing, creating a disembodying sense of scale and spatiality. However, Patricia actively engaged with social mobile media, especially the news.

Damien Williams, Patricia's 30-year-old son and Andrew, his 40-yearold partner, lived with Patricia in the same household. Andrew was the official IT person of the household, who installed and de-installed apps on everyone's mobile media. Both Damien and Andrew loved locative media and used it in many day-to-day scenarios. In particular, Andrew even had a dog locative media app that allowed him to track where the dog was in the house when he was at work - affording a particular type of care at a distance and friendly surveillance. Damien was an avid social media user who often tagged locations and time-stamps daily. His screens were dirty and broken from constant use and exposure.

This is in sharp contrast to Susan Jones whose screens were untouched by hands. Susan, a 65-year-old retiree who also lived in the house, was starting to get acquainted with mobile media by doing iPad classes. She would not

Hjorth, L., K. Ohashi, J. Sinanan, H. Horst, S. Pink, F. Kato, B. Zhou, Digital Media Practices in Households: Kinship through Data. Amsterdam: Amsterdam University Press, 2020 DOI 10.5117/9789462989504_CHO5 


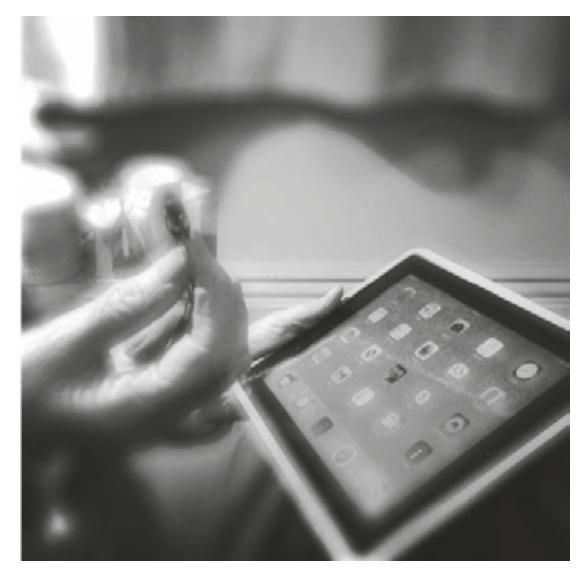

Figure 5.1: Susan using her iPad pointer (so her fingers don't touch the screen)

touch the screen with her hands, instead using a special pen pointer that she bought after seeing one of her iPad teachers using it (see Figure 5.1).

The "Williams" family is indicative of the multiple ways in which locative media, as part of broader social mobile media, was and was not being used in Melbourne households. We found generational and gendered differences playing out, both in and around their relationships to embodiment and notions of place. As Patricia's example highlights, the experience of place through paper maps creates a different sense of scale and movement that is constant, whereas the movement with Google Maps gives a sense of inertia and disorientation. Locative media use and non-use moves in and out of household rhythms - as symbolic of the screen's relationship to embodiment and place. For some participants, screens are so much part of our lives that they reflect the messiness of life through dirt and scratches. Other screens are viewed as a continuum of older screen practices whereby it was seen as bad to touch screens, such as TV screens.

The rise of haptic playful screens and media practices requires us to revise our methods. What does the dirty screen mean to a sense of embodiment? And does this differ, if at all, with the screen that remains untouched? What does it mean to engage with the intimate and haptic dimensions of media practice? What does it mean to study mobile media through knowing hands? What do the hands say that go beyond the spoken? Can we find similarities across cultural contexts? Or are there culturally specific gestures?

As we argue elsewhere, we need a theoretical interrogation of how we know through the hand, one which offers a deeper understanding of what and how we might learn through a tactile approach to digital ethnography (Keogh 2015). In other words, there is a growing need to understand screens 
as part of a haptic ecology that moves in, across and around the screen. We need to consider what researching through and by the hands might add to our methods and theorization for mobile media and screen research. These haptic practices inform how we interact, experience and understand locative media in our everyday.

In this chapter we develop this line of investigation through our crosscultural studies of locative media in and around the family. We consider how taking a tactile digital ethnography approach (Pink et al. 2016) might teach us new things about how we can study the practice through the "knowing" hands that might express tacit meanings. These meanings can be generational, technological affordances, culturally specific and also arguably innate gestures. In particular, we consider through this approach what "knowings" are culturally specific and which ones are more generic in and around the mobile screen. This study did not intentionally begin as a study of the hands, but through the digital ethnographic methods and the process of ethics where we wanted to de-identify our participants, filming in and around the hands and screen became increasingly prevalent.

\section{Reading Gestures}

Game studies have a long history in understanding the importance of touch as a sense of embodiment. As Brendan Keogh $(2015,1)$ observes in his phenomenology of videogame play, that there is a need to attend to the multi-sensorial dimensions of play through, in and around screenplay. Keogh argues, "Through an entanglement of eyes-at-screens, ears-at-speakers, and muscles-against interfaces players perceive videogames as worlds consisting of objects and actors with texture, significance, and weight" (Ibid.). Game consoles such as Nintendo Wii have been important in understanding and addressing the multi-sensorial experience of play in and around the screen-much of the work discussing this console speaks about the excessive and in-excessive deployment of the gesture (Giddings 2014). Simon (2009) argues that there is a "gestural excess" present in all videogame play that has been harnessed by haptic technologies such as Nintendo Wii and, more recently, touch screen play.

As the work of David Parisi (2009) discusses, it is important to create a media archeology of the touch in order to understand more deeply the relationship between touching and media. Touching did not just become important with smartphones. Parisi notes through the example of the Nintendo DS, the advertisements had to teach people to learn to touch the screen after decades of etiquette not touching the screen. It is important 
to remember this history when considering the normalizations around touching screens. They are generational and they are cultural. They are taught, untaught, retrained.

Parallels to the rise of the touch and screen-and its impact upon the spatial dynamics and context outside the screen/media-can be made with the rise of personal and portable technologies. For example, Rey Chow (1999) in her important study of the Sony Walkman in China argued that the technology revolutionized the role of listening. In China, listening had been historically a collective and public activity. Through the Walkman, it became internalized and personalized. Alternatively, Japan has a long history of the personal and portable as argued by Ito, Matsuda and Okabe (2005). The focus upon contemporary media as participatory needs to engage with the fact that participation takes many forms-including listening and the tacit.

The role of gestures in and around portable media has its history in newspaper and novel cultures. Reading traditionally in Chinese and Japanese required readers to begin at the Western "back" of the book and to move backwards to the front. In its adaption to touch screens (mobile and tablet) there has been some re-choreographing of the hand and reformatting of the book. In a western context swiping might involve the flicking from right to left for "page turning," which has then been adopted into eastern contexts. It is significant to recognize that these gestures are not innate but historically specific.

In this chapter we consider some of the ways in which haptic play moves in and around the attendant screen cultures. What we see is that the haptic quality of the screen creates different techniques and affective practices across cultures in the three locations Melbourne, Tokyo and Shanghai. We firstly define haptic play and its relation to the screen as defined through fieldwork. We see how affective labor through emojis can create different playful approaches to intimacy in and around the screen.

\section{Haptic Play and Screens}

It's a personal record. I enjoy the moment of check in. It's like treasure hunting. I would feel frustrated if I couldn't check in because of lack of WiFi connection. I don't want to socialize on Swarm. It's my own world. (22-year-old Yuto)

As Yuto noted, locative media game Swarm provided him with a playful way in which to think about his movements. He gained a sense of joy, much 


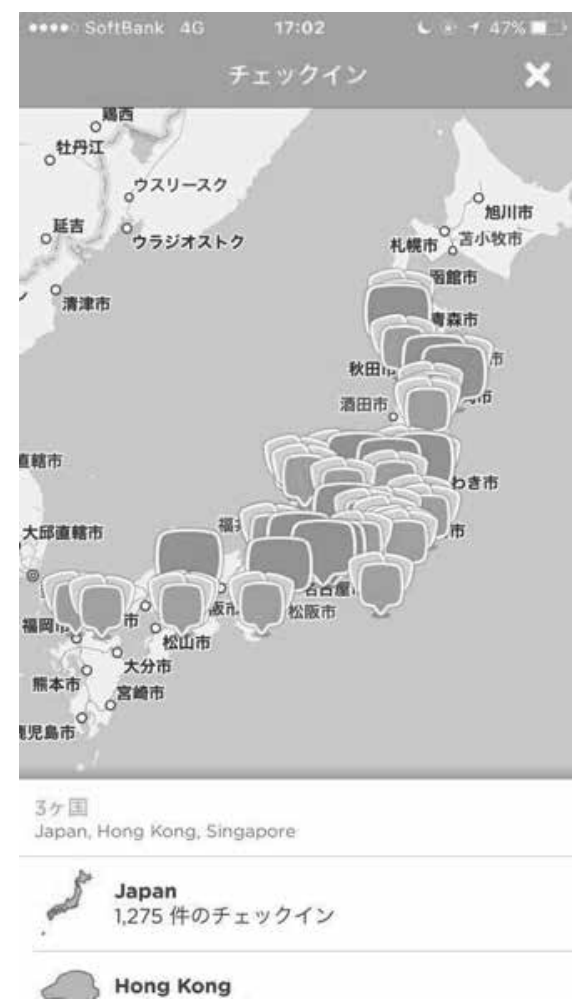

Figure 5.2: Yuto's journey via Swarm mapping

like a game reward, when viewing his journeys via Swarm. As a 22-year-old undergraduate student, Yuto moved from the northern part of Japan to Tokyo to enter the university. Yuto lived with his father (52-year-old), who worked in Tokyo, while the rest of his family - his mother (52-year-old) and his younger brother (17-year-old)—continued to live in his hometown. He bought his iPhone before entering the university and downloaded 10 apps including Twitter, Facebook, Instagram, LINE and Swarm.

Yuto used to tweet on a daily basis, but at the time of our research he did not tweet anymore because he had started job hunting and was worried some companies would judge him via his tweets. After that he made his Twitter account private. Yuto's media practices changed dramatically when he met his girlfriend and started to feel that he did not need to engage with social media, from then on only using it as a personal archive. Yuto's favorite app was Swarm, which he checked in on almost every day.

In the example of Yuto we see how media can be deployed in different ways to create a sense of haptic play, which moves in and around the screen (Figure 5.2). For Yuto, haptic play occurred through his deployment of Swarm 
as a form of a personal diary for mapping place. But it was also translated into other practices. For example, for Yuto, the sumaho (smartphone) was an extension of intimacy with his girlfriend, in the form of "girlfriend in the pocket." Yuto had his sumaho turned on and received alerts when his girlfriend sent him a message. He did not carry his sumaho in his hands but rather carried it in his pocket. These pockets become repositories for haptic play, awaiting his girlfriend's message. Haptic play moves in and around the screen and knowing hands.

Hjorth, Richardson and Balmford (2015) note in their study of mobile games how questioning seemingly trivial gestures in and around mobile media practices actually embodies a wealth of "deep" attitudes about privacy, boundaries between work and play, social etiquette, and care for the device. A "simple" gesture of having the phone screen face down when talking in face-to-face scenarios can reveal complex methods for negotiating engagement between online and offline worlds. As they note "it is clear that the bodily methods of interfacing with the materiality of the screen are of paramount importance to participants' engagement." The notion of haptic play seeks to engage with these new "techniques of the body" that must be accounted for if we are to interpret the complexities and intentionalities of use.

In the context of mobile touchscreens, it is not that such devices demand a more embodied or sensory mode of interaction, but that they have "alerted us to the sensoriality of our embodied and affective engagement with media in new ways" (Pink 2015, 6). As we explore the mutual imbrication of media interfaces and our embodied selves, the intimate connection between perception and meaning that is always-already both individual and collective.

Paterson $(2007,1)$ explores both the historical and contemporary theorizations and dimensions of touch. He argues that touch cannot simply be defined in physiological terms; it is also always "a sense of communication", and more significantly, it is manifold. This turn to "touch theory" is complexly interwoven with the emergence of haptic technologies and, in media studies, is often applied to the analyses of computer and touchscreen interfaces. In Paterson, Dodge and MacKian's (2012) terms, haptic media engage the manifold facets of touch, a sense ensemble that incorporates cutaneous, kinesthetic, proprioceptive, somatic, mimetic, metaphoric and affective modes of perception. Over the past decade such analyses of the haptic interface have enabled fertile connections between media theory and the disciplinary fields of sensory studies and new materialism. 
Parisi $(\mathbf{2 0 1 5}, 6)$, for example, traces an "intensified focus" in game studies on the way digital games invoke and depend on the body's movement and perception, which has followed the success of gesture controls and haptics (such as vibrating or rumbling controllers, or mimetic devices and applications such as the Nintendo Wii and many mobile games). In response, researchers have developed "nuanced theoretical frameworks capable of accounting for the body's newfound centrality to the play experience." Just as Parisi demonstrates the ways in which mobile gaming consoles advertisements sought to educate users about touchscreen interfaces after years of non-tactile screen cultures in the form of TV, so too can we see that the "intuitive" nature of touch and play is actually culturally and historically situated.

Sicart $(2014,22)$ distinguishes between play and playfulness. He has taken on the notion of "playfulness" as an attitude, an orientation to an activity, which also characterizes contemporary media. This attitude is not specific to games but can be seen in the seamless integration of games and creative mobile apps into our everyday lives and modes of communication, the perpetual expansion of participatory media forms, and in the rise of ever evolving social media services that enable users to upload, share and remix their own and others' creative "small media" content.

Exploring playful practices and the lusory attitude from the position of games gives us different ways to think through the role of data and algorithm in media cultures, from the quantified self $(\mathrm{QS})$ and gamification, to virtuoso play, creativity and playful resistance. Here the playful can be seen as an orientation to action, a mode of inquiry, and a set of practices that can help to expose some of the tacit power relations in and around the rhythms of data in everyday life. Play is fundamentally a creative, political and social activity.

\section{Haptic Rhythms}

Haptic interfaces can provide new experiences for and of the screen within everyday contexts. These haptic rhythms can create a sense of intimacy and temporal urgency that might not happen with non-haptic sense. Haptic screens provide a greater embodiment by deploying not only the sense of visual, but also, more importantly, the often-tacit sense of touch. The role of touching is culturally specific as well as historically informed. Touching involves a type of embodied sensorial experience. As noted with Yuto, haptic media in the form of vibrating alerts from the girlfriend in his pocket created 


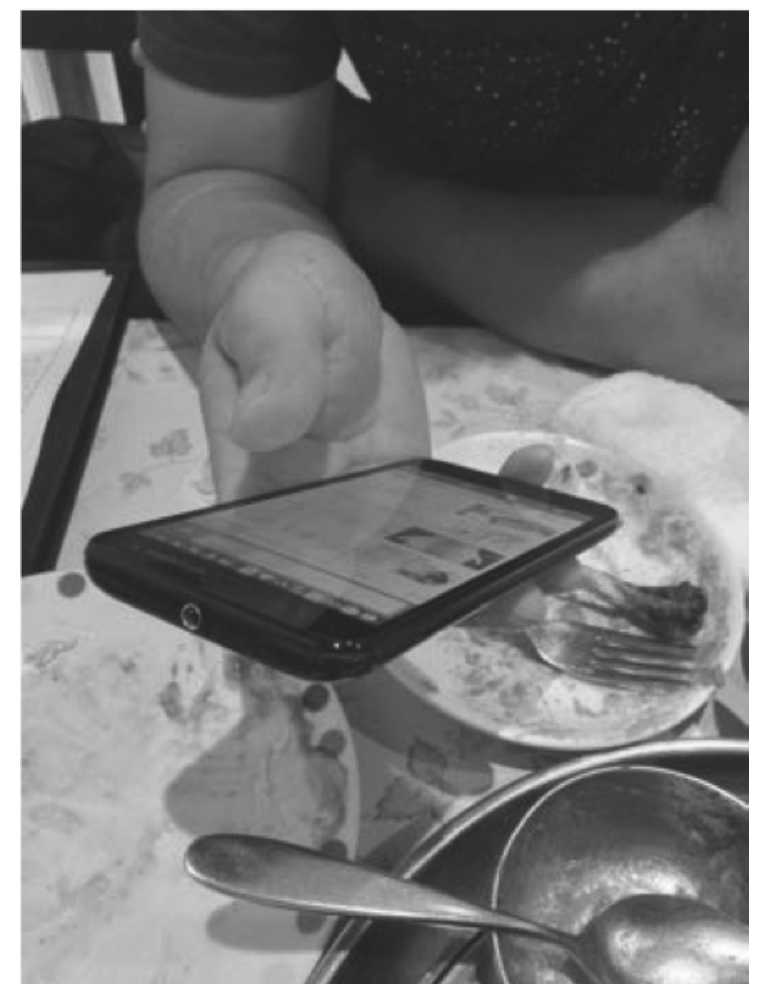

Figure 5.3: Yuto's smartphone was often in his pocket. He took it out during the interview.

a sense of intimacy as well as urgency (Figure 5.3). When Yuto received the vibration in his pocket he brought it out and quickly replied. Then he placed it back in his pocket. He was unaware of other people's messages as the phone was hidden in the pocket.

For Mana, the haptic nature of the mobile screen meant that she and her mum were so quick in their responses throughout the day that they were almost instantaneous. When Mana received the vibration that her mum had sent a message, she replied straight away. Mana had recently experienced some changes in her life that had made her exchanges with her mum even more frequent.

One year before our research, Mana broke up with her boyfriend of ten years. This made her quit her job in a local region and move to Tokyo for a new job. In order to try to "move on" from the heartbreak, Mana moved into a "social apartment" in the hope of meeting new people. A "social apartment," often referred to as "interactive rented accommodation" has a kitchen and lounge on the first floor where residents can contact and communicate 
with each other and associated individual rooms. Social apartments are a new phenomenon in Japan whereby companies buy an apartment and the employees can live there. They are, according to one advertisement, making "your life more open and connected." Here social refers to "social" in media rather than more historical or political notions of social. In places such as this, the friends become symbolic of the "family."

As Mana got used to the life in the apartment she began to think about the residents as akin to a family. As she described, "when I leave home they say, 'have a nice day' and when I go home, they say, 'welcome back'." Someone is always in the lounge. Someone receives a package when I am not there and if someone cooks or I cook, we eat together." Her "real family" lived in Tohoku (northeast) area. Her family included her parents, her elder sister, sister's husband, their child, her younger brother, brother's wife and their two children. As Mana lived apart from the rest of her family members, she made efforts to reply to messages on their LINE family group as soon as possible, using situational stamps such as "Cute!" or "Take care!" to create "a sense of participation." She bought different kinds of stamps to make her family laugh.

Takeshi, a 27-year-old hospital employee, lived with his family—his father (61-year- old), his mother (61-year-old) and his elder brother (31-year-old). He started to use a smartphone in 2012. His most used social media were LINE and Facebook, which he used to contact his friends. He also played games while using e-mail to communicate with his family. His favorite game at the time of our research was LINE PokoPoko puzzle game. In the game, the main character, Boni, and the other residents of Poko Forest must save the forest from the evil demons and return peace to their home by getting high scores on each stage and restoring the forest to its natural state by making the flowers bloom. The game, typical of the haptic play of contemporary mobile games, is as follows.

Each time you play the game, you consume one "clover." Clovers are like tickets necessary to play the game. Clovers regenerate at a pace of one every 30 minutes. Takeshi usually played the game for five minutes and consumed five clovers before going to work in the morning. By lunchtime, the clovers had regenerated. The rhythm of the game perfectly reflected his working hours. When we interviewed him in 2017, he had recently started to use another game app, Shogi Wars. Shogi is Japanese chess and in Shogi Wars, you can play with other players online. In Shogi Wars there is a "10SEC" mode that allows 10 seconds per move.

In Shogi Wars there is a "10SEC" mode that allows 10 seconds per move, which Takeshi played when he was with his family or his girlfriend after 


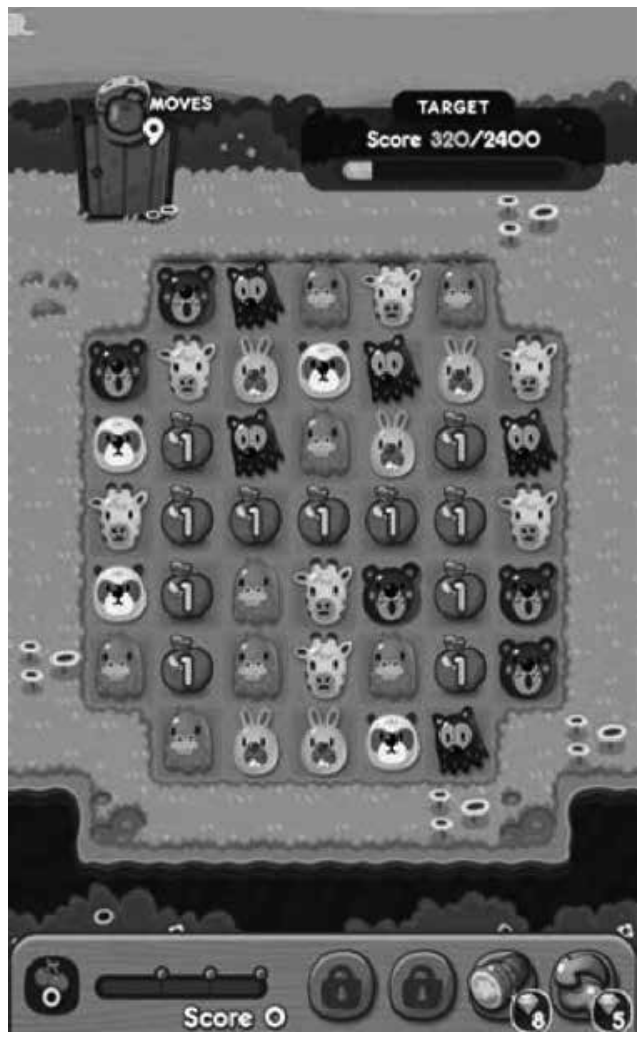

Figure 5.4: LINE PokoPoko

work. "In this mode, you can see the result of the match right away. So, it doesn't bother communication with others. I like to play this game under the gaze of my girlfriend," Takeshi explained. For Takeshi, it was his right thumb that was automatically deployed when using mobile touch screens. The impact of such haptic play exercise can be noted in the fact that his right thumb was bigger than his left thumb.

Within our Shanghai and Melbourne fieldwork, parallels and differences could be found. In Shanghai, unlike the traditional in-person family communication, family intergenerational communication via WeChat provided a more relaxing and playful format. The playfulness Sicart (2014) has suggested is prevalent in contemporary digital media is affording Chinese family's new ways of conversing in everyday scenarios. As parents of young adult children become more immersed within digital media and the attendant forms of unofficial literacies, the children are the ones being surprised by their parent's playful use of media. We see new forms of depth in terms of what haptic play performativity entails. 


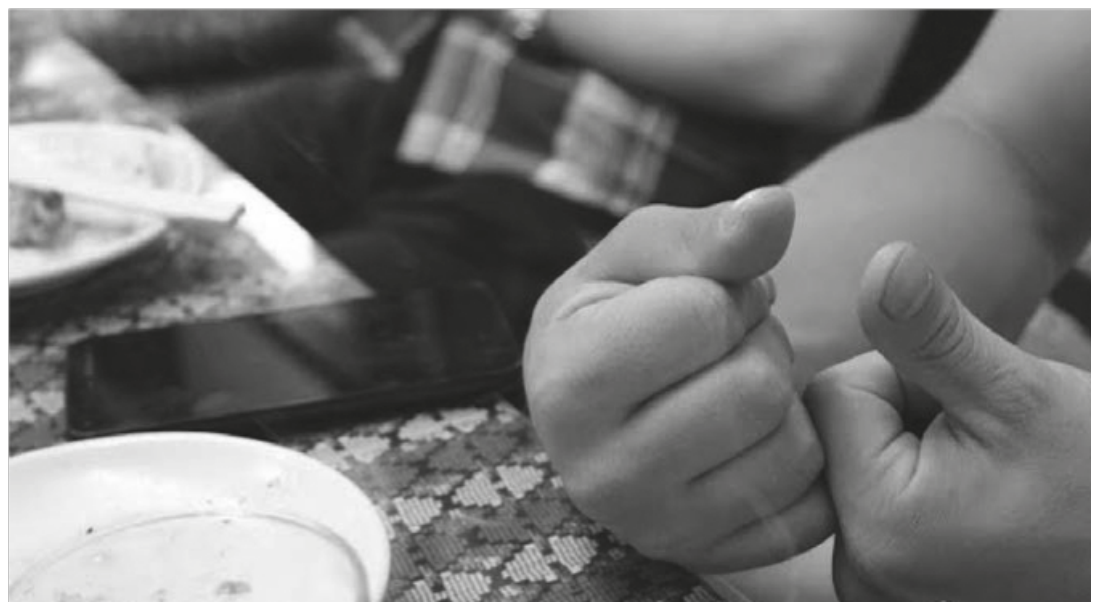

Figure 5.5: Takeshi's right thumb became bigger than his left thumb

\section{Haptic Play Poetry}

One such incident is the now famous case (see Figure 5.6) of a short poem written by a mother to her daughter on WeChat and then shared by the daughter on Weibo.com. During April of 2015, this WeChat screenshot rose to popularity and was shared by thousands on Chinese social media. The poem, taking the format of the WeChat message, roughly translates as,

Dear daughter Yaya/It's already spring time/Let's go on a trip/I'll go with you/and you'll bring the money/No matter where we go/Mount Tang-la is fine/the Desert Tengri is also good/Let's go on a trip at once/As long as we set off/I'll go withyou/andyou'll bring the money/YOU MUST REMEMBER TO BRING THE MONEY!!!

The daughter, Yaya, found the poem playfully humorous and so she posted it on Weibo (see Figure 5.6), which triggered a wide range of online responses. The Weibo post was forwarded over 19,00o times and the mother became famous, in an interesting way.

According to Yaya's other updates, her mother was very fashionable amongst her peers and was a savvy user of digital media like WeChat. The mother was very pleased about her impromptu internet fame and even has a post in her personal WeChat Moments to celebrate the phenomenon. This story is but one example of how WeChat's popularity across the generations is creating playful entanglements between generational differences in expectations and modes of use. In our interviews with parental participants, 


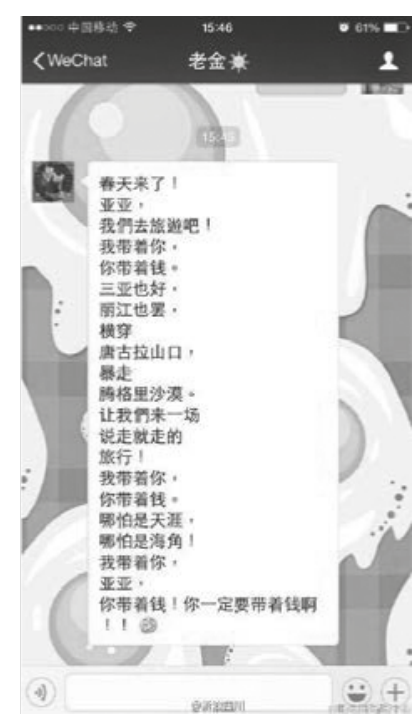

Figure 5.6: A mother's poem shared by her daughter on Weibo.com

"play" was the actual word (wán) used frequently to describe their daily involvement with WeChat. As a communication tool that connects friends and families, even strangers, together, WeChat was viewed as a useful "toy" to "play with." These digital immigrants explore WeChat in a playful way, enjoying sharing photos, sharing posts and sending stickers.

This playfulness is not only thanks to the rich media formats available on WeChat, but also due to plethora of interesting WeChat emoticons and stickers. There are two default kinds of emotional icons in WeChat - the old emotional icons derived from the first-generation of mobile social media, QQ, and emoji.

According to screenshots gathered from all participants, it became apparent that parents use the emoticons more often, while the children prefer using emoji. In addition, WeChat allows stickers that provide different ways to express feelings playfully. Here there are distinct parallels between generational use of WeChat in China and LINE in Japan. In WeChat there are two kinds of stickers - those provided by WeChat and users' customized stickers.

In Figure 5.8 we can see eight cartoon icons indicative of the WeChatprovided stickers. Within the WeChat sticker format there are those that are free and those that can be purchased. WeChat also encourages users to upload their customized stickers. In fact, customized stickers have gained much popularity among young WeChat users. Popular customized stickers change along with online hot topics. By using interesting stickers, dialogue on WeChat is textual, but also visual, emotional and playful. 


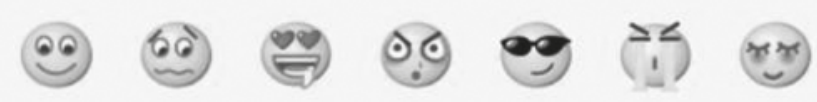

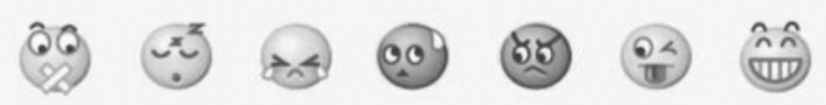

(30) :28) (20) $\times$

- 0 O 0

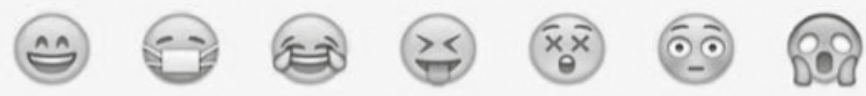

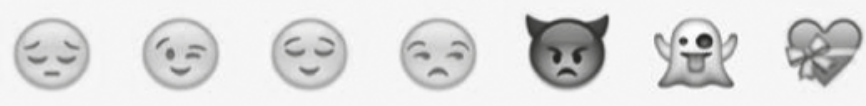

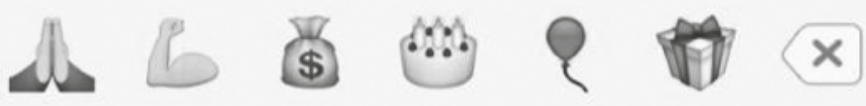

Figure 5.7: WeChat emoticons
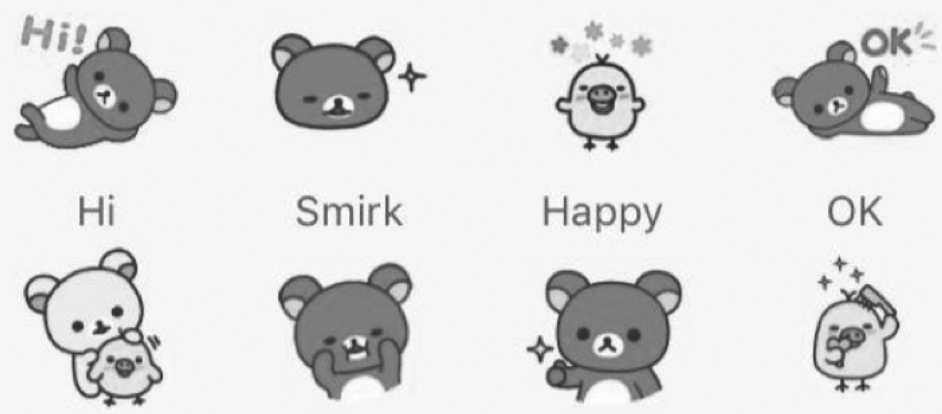

Smirk

Happy

OK

Praise
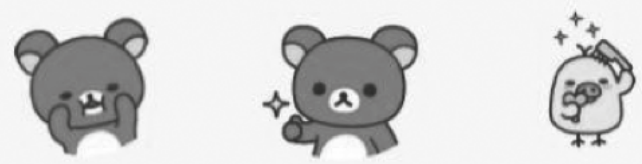

Silly

Good

Groom

Figure 5.8: An example of WeChat-provided stickers 


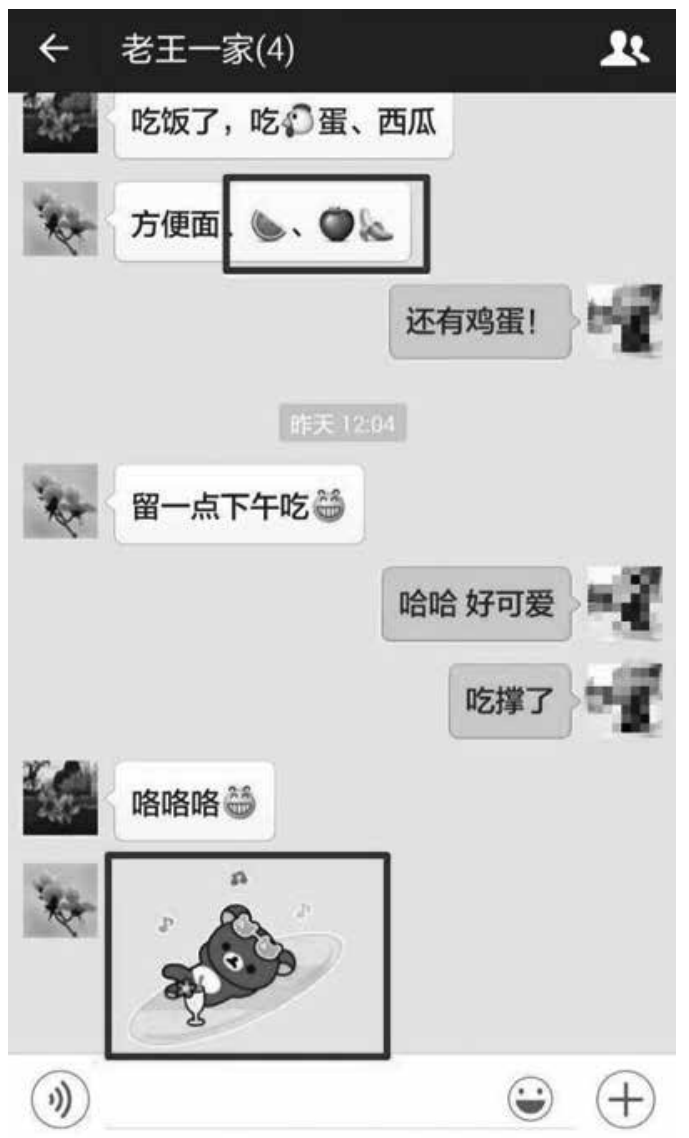

Figure 5.9: Screenshot of Taylor's family WeChat group

\section{Haptic Play Cadences (Co-present Frequency)}

In order to understand the emoticon phenomenon further, we examine the example of Taylor and her familial deployment of haptic play through frequent use of emoticons. A 24-year-old master's student living in Shanghai, Taylor left her remote hometown for University when she was 18. According to Taylor, WeChat had now become a significant communication channel for her family. She frequently used text, audio, and the Moments function in WeChat to interact with her parents. It was under Taylor's initiative that the family created a WeChat group for her parents to correspond with her frequently.

In Figure 5.9 we can see a screenshot from an interaction between Taylor's family members on WeChat. They were talking about lunch. Taylor's parents 


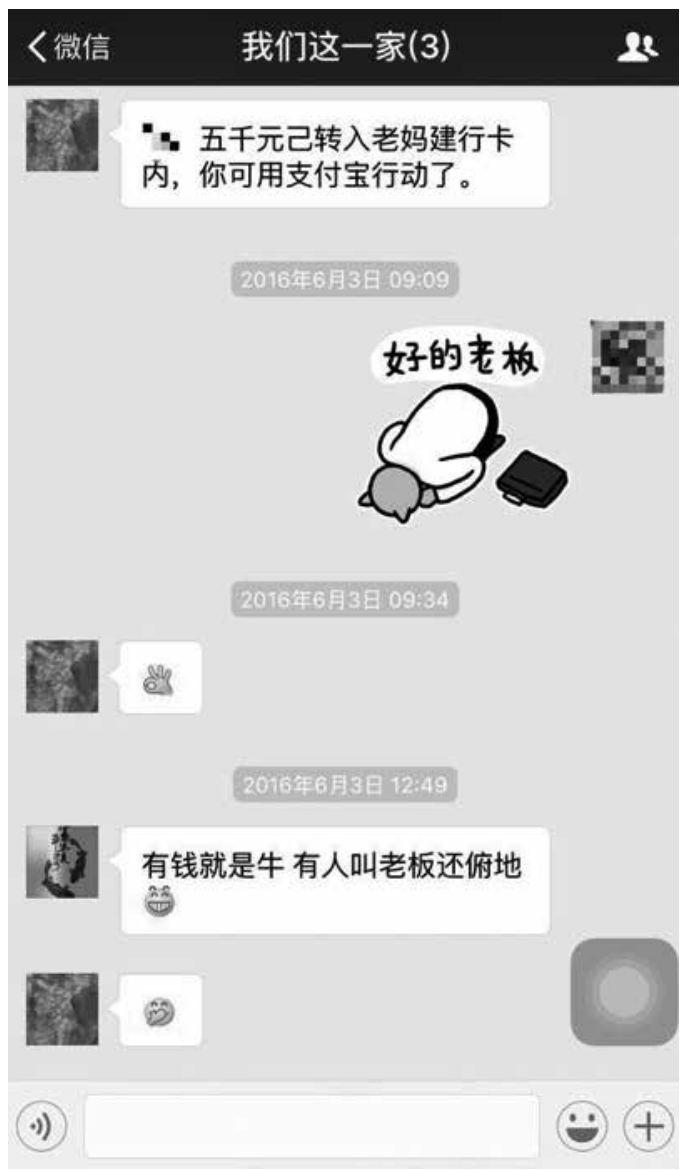

Figure 5.10: Screenshot of Tiffany's family WeChat group

deployed a range of emoticons and stickers to convey certain information and-more importantly - to express certain feelings. Through the use of a tapestry between emoticons, emoji and customized stickers, mundane familial interactions can be given a sense of emotional texture and haptic intensity.

In another case we find Tiffany. Tiffany is a 24-year-old master's student in Shanghai whose parents live elsewhere in China. She had also established a WeChat group for her family. According to Tiffany, though her parents did not have much knowledge of digital culture before she left, they had fully immersed themselves in the practice at the time of our research.

In Figure 5.10 we can see a screenshot of her parent's skillful and complex deployment of emoticons against Tiffany's minimal use of one sticker. What is interesting is that Tiffany chose a sticker meaning "OK, Boss!" however she 
was at first only able to express the meaning "OK." (When user types in "OK", this "OK, Boss!" sticker would automatically pop up as the recommended sticker, as long as the user has downloaded that set of stickers.) Tiffany noted, "It's really funny that my parents tried their best to understand the literal meaning of the sticker and replied with their illustration. That's really cute." As Tiffany's exchange with her parents demonstrates, phatic and mundane talk can be transformed into playful exchanges.

In Melbourne, we also found that family members of different generations can create a feeling of playfulness and closeness through using stickers and emojis. Nancy, who lived with her 6o-year-old husband and 30-year-old daughter in the outer western suburbs of Melbourne uses WhatsApp to chat with her cousin, nieces and nephews in Singapore a few days a week. She said, "I think they see a different side to me when we chat, it's not like I'm just the strict aunty when I visit." When her cousin or her niece wanted to have a more serious conversation with her, her cousin would Skype her and they would have a face-to-face conversation for up to an hour and her niece called on her iPhone using Skype or WhatsApp. For conversations that were more of a simple update or checking in, they would exchange quick texts over WhatsApp and Nancy always ended the exchange with a 'thumbs up,' high five or smiling emoji.

Nancy described how she picked up using emojis from her niece and nephew, since "they are always up to date with gadgets." For Nancy, WhatsApp was all about sharing emojis as part of an intimate co-presence. By using emojis she thought were cute, Nancy saw emojis as way of communicating intergenerationally - especially with younger members of her extended family in a way that was more familiar and colloquial to them (see Chapter 2). Through emojis she could create a relationship more akin to a friendship, rather than a strict aunty. Nancy's sister-in-law Esther lived 20 minutes from her with her husband, Patrick. Their daughter, Jasmine, lived with her husband in the inner northern suburbs of Melbourne. Jasmine saw her parents around once a week and the rest of the time, her primary communication with Esther was over Facebook Messenger.

Similar to Nancy, Esther was introduced to using emojis and stickers by a younger member of her family. Jasmine had been chatting to her mother and when she said she had to say goodnight as she had to wake up early in the morning, she followed the statement with a sticker from Facebook's Cutie Pet series of a cat crying. Esther exclaimed, "That's so cute! How do you do that?" and Jasmine explained how to access the sticker store. Within a few minutes, Esther had browsed the store and downloaded a few sheets 
of stickers. This type of action-based learning is synonymous with social media communication.

During our earlier visits to Esther, she commented that she rarely checked her phone when she arrived home from work-often missing calls and texts from Jasmine as her phone was still on silent from her workday. Esther's main reason for avoiding checking her devices at home was because she sat in front of a computer all day at work, so when she arrived home, the last thing she felt like doing was being tethered to yet another piece of technology. Jasmine also explained that she found contacting her mother extremely frustrating, as her mother never checked her phone. Given this issue Jasmine switched to Facebook Messenger as her primary source of contacting Esther.

Esther often ended her day by playing solitaire or reading on her tablet and when she turned it on, the tablet automatically updated her with notifications of messages received, so Jasmine was sure Esther would know when she had tried to contact her. Secondly, with stickers, Jasmine didn't just feel like she was overloading her mother with logistical information, about plans for the weekend or other kinds of coordination. By leaving a sticker at the end of the exchange, both mother and daughter felt like they had also left the conversation with something light-hearted, playful and cute.

\section{Conclusion: Playful Encounters}

As noted earlier, contemporary digital media has been defined as playful (Sicart 2014). Play and emotional gesturing can occur inside and outside the phone and is key to understanding the affective sharing of intimate co-presence. They help to reinforce and transform digital kinship ties. Through emojis, playful emotions can be shared without requiring faceto-face contact. Through emojis, intergenerational communication can be fostered in creative and inventive ways.

Both play and personalization have a particular genealogy in the mapping of digital media in the region. The role of personalizing digital media with emoticons (i.e. emoji) has a long history in Asian countries such as Japan, whereby the role of the cute (kawaii) plays into subcultural practices that seek to question traditional gender and age prescriptions, as noted in Chapter 2 and further discussed in this chapter. With the uneven uptake of mobile and locative media in the region, localized forms of cute character culture have become more prevalent across the generations. Grandparents, parents and children can be all found deploying emojis, emoticons and 
stickers in playful ways that are both tied to generational norms as well as providing new ways to transgress intergenerational boundaries.

In this chapter we have explored some of the ways in which haptic play is becoming a key part of everyday mundane intimacies between the generations. The affordances of the haptic and playful media like emoticons is creating ways in which generations are challenging norms around notions of media literacy. These are indicative of the types of digital kinship emerging in the three locations.

\section{References}

Chow, Rey. 1999. "Listening otherwise, music miniaturized: A different type of question about evolution." The Cultural Studies Reader, edited by Simon During. 382-399. London: Routledge.

Giddings, Seth 2014. Gameworlds. London: Bloomsbury.

Hjorth, Larissa, Ingrid Richardson, and William Balmford. 2016. "Careful surveillance and pet wearables: At home with animals.” The Conversation, 5 September. https://theconversation.com/careful-surveillance-and-pet-wearables-at-homewith- animals-63883. Accessed 20 May 2017.

Ito, Mizuko, Misa Matsuda, and Daisuke Okabe. eds. 2005. Personal, Portable, Pedestrian: Mobile phones in Japanese Life. Cambridge: The MIT Press.

Keogh, Brendan. 2015. "Paying attention to angry birds." The Routledge Companion of Mobile Media, edited by Gerard Goggin and Larissa Hjorth. 267-275. New York: Routledge.

Parisi, David. 2009. Game Interfaces as Bodily Techniques. New York: IGI Global.

- 2015. "A Counterrevolution in the Hands: The Console Controller as an Ergonomic Branding Mechanism.” Journal of Games Criticism 2 (1):1-23. http:// gamescriticism.org/articles/parisi-2-1. Accessed 20 May 2016.

Paterson, Mark. 2007. Senses of touch: Haptics, affects and technologies. London: Berg. Paterson, Mark, Martin Dodge, and Sara MacKian. 2012. "Introduction: Placing Touch within Social Theory and Empirical Study." In Touching Space, Placing Touch, edited by Mark Paterson and Martin Dodge, 1-28. Surrey: Ashgate Publishing.

Pink, Sarah. 2015. Doing Sensory Ethnography. London: Sage.

Sicart, Miguel. 2014. Play Matters. Cambridge, MA: The MIT Press.

Simon, Bart 2009. "Wii are out of control: Bodies, game screens and the production of gestural excess." Canadian Games Studies 3 (4): 1-17. http://journals.sfu.ca/ loading/index.php/loading/article/viewArticle/65. Accessed 26 May 2016. 


\title{
Section III
}

\author{
Visualizing Kinship
}





\title{
6. Personal Visual Collecting and Self-Cataloguing
}

\begin{abstract}
Chapter 6 analyzes the growing role of the visual in social media practices in terms of tensions between sharing, impression management and self-cataloging.
\end{abstract}

Keywords: sharing; impression management; self-cataloging

In this section of the book, Visualizing Kinship, we explore the particular role of camera phone practices in kinship and family relationships through its aesthetics of mundane intimacy and co-presence. With the rise of algorithms, automation and movements such as quantified self (QS) and attendant methods of self-tracking (Lupton 2016), the role of documenting one's life through different methods have come to the forefront, many of which involve forms of visualization, especially through camera phone use (Pink and Fors 2017a, 2017b). As Humphreys notes, contemporary digital media practices are increasingly about not just quantifying but qualifying the self through creative data archiving and sharing (2019). However, as we will explore later in the book's last section, Co-futuring Kinship, the ramifications of progressively more datafication in everyday life has yet to be fully realized in terms of digital legacy and digital health.

Against this trend, some are opting out of the politics of sharing digital data and instead focusing on representing the ephemeral (such as Snapchat or non-sharing). In these paradoxical trends, camera phone practices are integral. The differences between sharing as a collective memory and nonsharing as a form of diarization play out across cultural and generational divides. This tension between the archive and ephemeral media is at the core of theories around photography and has been harnessed by social media like Facebook "memories" and Instagram "stories."

Hjorth, L., K. Ohashi, J. Sinanan, H. Horst, S. Pink, F. Kato, B. Zhou, Digital Media Practices in Households: Kinship through Data. Amsterdam: Amsterdam University Press, 2020 DOI 10.5117/9789462989504_CHO6 
In conceptualizing Visualizing Kinship, the entanglements between camera phone apps and geo-tagging practices are key. With the rise of geo-tagging camera phone apps, the role of emplacement becomes pivotal. As Hjorth and Pink (2014) note, time and place are emplaced on images through geotagging - creating a flattening of some dimensions in preference for others. This emplacement of locative media within the image creates particular cartographies for memory for the user and their intimates. Camera phones demonstrate that co-presence is reflected in the rhythm and movements across places, spaces and temporalities. Just like intimacy, presence has always been mediated, if not by technologies, then by gestures, language and memory. For Licoppe $(2004,135)$, mobile technologies "provide a continuous pattern of mediated interactions that combine into "connected relationships," in which the boundaries between absence and presence eventually get blurred". Mobile communication inflects presence on diverse levels in which "the ways absent ones make themselves present have been many" and that "copresent interactions and mediated communication seem woven in a seamless web" (Ibid.).

Within current camera phone trends, a tension between the archival and ephemeral relationship can be found. In much of earlier camera phone practices, people often unintentionally archive, taking and storing images of their everyday lives that they may or may not revisit. For first-generation camera phones, without the instantaneity of the app ecology (known as second-generation), sharing was a considered and deliberate act that took time and energy through sites such as Flickr. As Søren Mørk Petersen (2009) notes, digital photo sharing partakes in the logic of "common banality." For Ilpo Koskinen (2007), camera phones amplify a particular type of banality. Mundane co-present intimacy becomes a key motivator within this first-generation of camera phone sharing.

In second-generation camera phone practices, temporality between the act of taking and reflection on sharing is accelerated. It's easier to share than to not share (van Dijck 2008; Holland 2015). Motivations in and around the photographic act are more fleeting, with many of the affordances of camera phone apps seemingly leaning towards a compulsion to share. Scholars such as van Dijck have highlighted (2007), the logic of sharing is the default function for much of social media. For van Dijck in the Culture of Connectivity, sharing has become the "social verb" (2013). Expanding on this idea further, Nicholas A. Johns argues in Age of Sharing that sharing is central to how we live our lives today-it is not only what we do online but also, a model of economy and therapy (2016). Tracing across these areas Johns highlights how sharing can be understood as part of caring practices and discourses.

Moreover, with default time-stamps and geo-tagging, many camera phone apps like Instagram become intrinsic to the process of narrating everyday 
life. Through these moments we create micronarratives of the everyday, which, in turn, create forms of collective memorialization (van Dijck 2008; Sarvas and Frohlich 2011). Apps like Instagram, with their archival-type affordances, create a type of witnessing. This witnessing can create social bonding at the same time as it socializes certain events and moments to the collective witness.

It is whether there is a compulsion to share and make collective these processes, or to compile as part of a personal narrative and catalogue, which interests us in this chapter. How do motivations around sharing play out in different cultural contexts to different social norms of repository and sociality? And how do tensions between the shared and non-shared - the compulsion to archive or not—reflect existing and emerging patterns around the mobile phone as a tool for sociality and self-cataloguing?

Within the politics of second-generation camera phone practices, the emphasis starts to move towards emplacement-emplacing the temporal, social, spatial or geographic (Hjorth and Pink 2014). And yet these emplacements oscillate between practices towards the archive and, conversely, the ephemeral. This tension can be seen in apps like Snapchat and more recently Instagram's "Story" feature that put the emphasis on the ephemeral media. And so how do these tensions between the archive and ephemeral, collecting and diarization, play out in the three sites? And what role does locative media like time-stamps play in the categorizing of this mundane and intimate media into a type of visualizing kinship?

In this chapter we explore the role of the camera phone not only as a portal for collecting, sharing and diarization, but also as a site for which tensions around memory making and temporality. Through the crosscultural discussion we will consider the ways in which collecting is viewed as both a group activity (i.e. networked and co-present affordances) and individual activity (i.e. diarization and self-cataloguing) and how these practices reflect culturally specific notions of the mundane and intimate. Through outlining specific examples, we demonstrate the ways in which camera phone sharing (and non-sharing) is playing out as part of impression management, co-present intimacy, and diarization.

\section{The Politics of Collecting: Sharing and Non-sharing, Group Archive or Self-catalogue}

Our focus on the act of collecting may point to the issues of emerging rules and conventions among friends and family members, and of individuals' 
attempts to manage their identity within the social relationships. Photo collecting can be understood as a form of talk through which one can present images of self to others, and to oneself (Holland 2015; Kuhn 2010). A smartphone is handy and useful "gear" for documenting mundane life. Given the convenience of taking, sending, and uploading the photos, the use of smartphones may increase one's opportunity to generate what Ken Plummer $(1983,2001)$ called "life documents."

In contemporary digital media contexts, Kato (2005) has repurposed this idea of life documents to make sense of mobile media practices within a sequence of daily events. By transferring photos directly, Instagram, for example, can be understood as a group-shared archive of visual notes. An individual can reflect upon their day by tracing the timeline. Also, because a person's friends' photos are weaved into his/her timeline, they can also learn about how others were doing at the particular moment of that day (Van House 2011; Holland 2015). Moreover, by becoming a tool for "life documents," mobile media like Instagram become part of a process for reflection and also encourage forms of self-reflexivity. The documented moments become sites for analysis and discussion. This, in turn, creates a different relationship to the everyday and vernacular media (Gómez Cruz and Lehmuskallio 2016).

Previous studies on first-generation photo sharing suggest that sharing photos over websites may influence the ways in which we understand each other (Kato and Shimizu 2005). In this study, researchers developed an experimental mobile blogging model (moblog) for pedagogical exploration by students. Set in the first-generation camera phone period (i.e. whereby images were transferred to the computer and then uploaded to the internet), the students deployed the camera phone as a researcher's tool for understanding how the everyday is framed, experienced and visualized. What images were uploaded reflected both upon the individual as well as the group. Students considered how these genres represented the group and also became a way to, in turn, understand themselves; in this way, the camera phone became a tool for reflexivity. At the crossroads of teaching students and researchers about ways in which to analyze the everyday, moblog was a valuable tool, eventually being known as the "mother of social media."

There are also historical instances of such research archives, perhaps the most notable being the Mass Observation (MO) project founded in 1937 by Charles Madge, Tom Harrisson and Humphrey Jennings in which analog cameras were used to document everyday Britain. Through the camera, practices and processes of the everyday could be given new forms of visuality and visibility. In particular, they used the camera to explore the social and cultural dimensions of the working class in emerging urban cultures-an 
area which had previously been given little visibility. In turn, MO provided new models for engaging participants and researchers in dynamic ways, leading to what would be understood as new British social documentary. It also had its critics, most notably those that argued that researchers were aestheticizing the lives of the working class rather than providing some form of intervention.

However, researchers such as Harrisson argued that by giving participants camera they were empowering them in new ways to document their life (Highmore 2002). It is against this backdrop that camera phone and the attendant forms of citizen journalism and affective witnessing needs to be understood. Similar criticisms about the aestheticizing of everyday life through camera phone photography can be found (Jurgenson 2012). Yet understanding these practices can provide profound insight into motivations around sharing (and not sharing) everyday moments, and how these become part of the affective witnessing processes (Reading 2015).

Camera phone practices can be framed as part of broader cultures of collecting, yet in doing so they create a specific relationship between the digital, archive and ephemeral (van Dijck 2008; Van House 2011; Broekhuijsen, ven den Hoven and Markopoulos 2017). While digital photographs tend not to take material photographic form, through the mobile device they take on a type of materiality in terms of not only remediating portable devices like the analogue photo album but also create a type of what Anna Reading calls "wearables." Here the relationship between the body, embodiment and data take on a complex entanglement that has both material and immaterial dimensions, or a kind of digital materiality (Pink, Ardèvol and Lanzeni 2016).

As Geismar argues, one of the powerful properties of digital photography is its ability for co-presence. In Geismar's anthropology of photography in locations such as Vanuatu, she considers the,

... structure, the reception and use of digital images of museum collections where many tensions arise between photographic imaginaries of evidence and indexicality in regard to digital images, and the ways in which the social experience of looking is increasingly understood to be part of the digital image (the hallmark of images embedded within social media which is an increasing part of museum digitization projects). In the conservative context of museum imaging, photography is still a primary reference for the authority of digital visualization. However, the opening up of museum collections to new communities of care and engagement allows us to understand digital images of museum collections within the context of a more affective sociality $(2015,306)$. 
The relationship between informal and formal ways of collecting is being transformed through social media practices, especially camera phone apps. It is not uncommon for museums to now consider how they install works to make them more "Instagramable." The more an installation looks good in a picture, the more the picture is likely to go viral. Outside the museum context, personal modes of collecting reflect particular forms of sociality as they conform to specific forms of collective taste (Broekhuijsen et al. 2017; Watkins, Sellen and Lindley 2015). In this way, camera phone practices as tools for replicating and analyzing taste cultures circulating within groups can provide much insight. Take the sharing of food; while food rituals are global, they are also localized in their practices, as we will see in the following sections. They reinforce notions of what Erving Goffman calls impression management at the same time as they allow new forms of front and back stage to play out.

Instagram, for instance, can be understood as a site for one's face-work (Goffman 1959). The notion of face refers to an image as self-delineated in terms of approved social attributes. Through the act of collecting and sharing a photo, an individual is trying to construct and maintain proper relationships with others (Rose 2012; Watkins et al. 2015). An individual's postings are not only displaying to friends/family members what he/she has seen, but how he/she understands the relationships. On some occasions, users may take and share photos for the sake of connected friends, other than for one's own natural interests (van Dijck 2008; Van House 2011). Decisions on whether or not to make the photo visible to others may also reflect one's understanding about the relationships.

On some occasions, individuals are guided by their own communication strategies in terms of what to make available, to whom, and when (Litt and Hargittai 2014). The act of collecting and sharing may no longer be a simple and instinctive reaction to what we encounter in our mundane experiences. Rather, it became an act of making careful decisions in selecting proper photos to be shared, to appropriate groups of connections, in a right timing (Thudt et al. 2016; Rose 2012). In this regard, the act of collecting and sharing can be understood as a moment at which one understands about oneself and the relationships with others are constantly negotiated (Uimonen 2016; Vivienne and Burgess 2013).

\section{Tokyo}

As aforementioned, in Japan mobile phones are used as a tool to connect with intimates, especially family members (Ito et al. 2005; Matsuda 2005). 
While the mobile phone has a longer history in Japan, smartphones started to become an integral communication tool among family members, not only through their provision of e-mail, SMS, and telephone services, but also as a portal to social media in the late $2000 \mathrm{~s}$.

As Richard Chalfen (2011) notes in his analysis of the "shinrei shashin" (ghosts in snapshots), the relationship between ghosts and photography in Japanese culture challenges conventional readings of the snapshot. Mobile media and especially smartphones further complicate this situation in networked visuality and the attendant forms of affective witnessing (Papailias 2016). While photography has a long and important history in the role of the family and memory at a global level, as Chalfen highlights, this phenomenon is particularly prevalent to Japan (2011).

As discussed in Chapter 2, since 3/11, Tokyo's use of mobile media has gone under serious recalibration. With growing distrust of broadcast media like NHK (after they withheld important information about the Fukushima nuclear disaster), many millions have turned to mobile media like Twitter, Line and Instagram to help negotiate a constant co-presence between friends and family. In particular, according to a Toyokeizai media report, 90\% of Instagram users are under the age of 35 and almost $70 \%$ of its users are female (Toyokeizai online 2014). Instagram has exploded in popularity in Japan since 2014, four years after its inception. Camera phone practices can be understood as a vibrant part of contemporary visual culture. In order to understand the gendered use of Instagram, we introduce three examples that highlight the functions and meanings of camera phone practices as a way in which to navigate the everyday, its rituals and co-present intimacies.

Mari was a 21-year-old Japanese graduate student. She bought a sumaho (smartphone) when she started at university and used LINE, Facebook, Instagram and Twitter. While she used LINE to communicate with her friends and family, Instagram - which she started to use the year before our research — was deployed the most to share photos with her friends. When she had time to spare, she opened Instagram and checked her friends' photos. As Mari described, "I share photos on Instagram to save my favorite moments and daily things. It's a kind of diary. Whenever I travel, I share photos on Instagram." She shared travel photos with location information and there were many photos of food and scenery on her Instagram. Instagram had effectively replaced Twitter and Facebook, which she had not updated for some time.

Mari was aware of her followers when she shared photos on Instagram. She didn't share photos if she thought that they were not interesting for her 


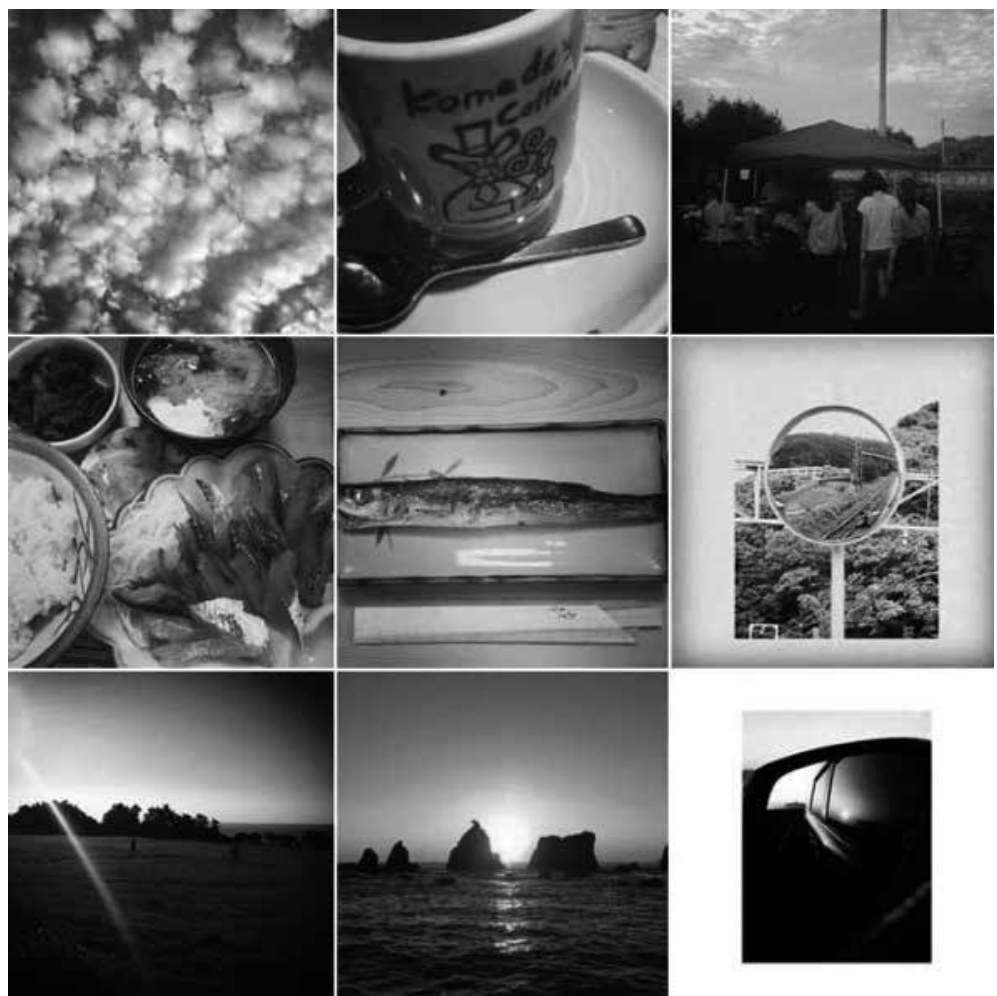

Figure 6.1: Mari's Instagram images

followers. For Mari the choice of what to share and what not to share involved maintaining a sense of intimacy through the mundane. As Mari notes:

On Facebook, there are too many 'friends' who are not my real friends. But on Instagram, most of the followers are my real friends. I would like to share my interesting experiences with them using Instagram. On Instagram you can show them what you saw and experienced.

Nozomi was a 22-year-old graduate student who started to use sumaho when she was a high school student. She used Instagram and LINE on sumaho. She had Twitter and Facebook accounts, but had not used them for a year. Although she had been an active user of Twitter and had more than 1200 followers, on seeing unpleasant tweets from her ex-boyfriend, she deleted the Twitter app. She found Facebook boring because people post only "official reports" on it. She used LINE, a Japanese social network site, to communicate with friends and family. 


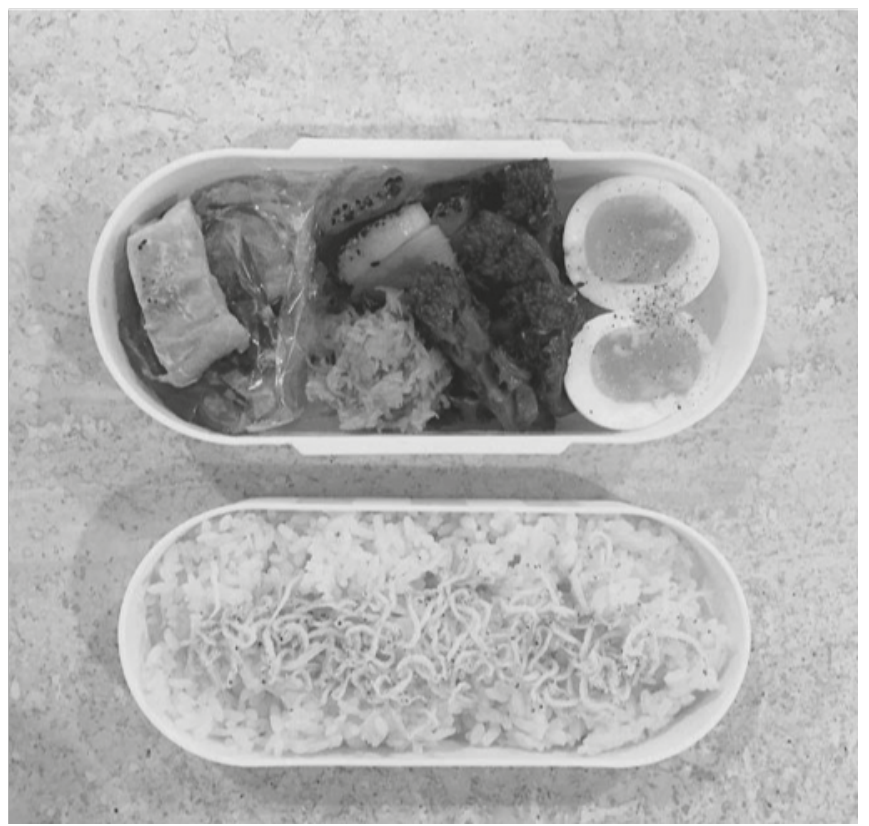

Figure 6.2: Nozomi's lunch photographed and shared

When it came to sharing photos, she used Instagram the most. She started to use it because of its editing features. "I was surprised. My photos became so cute with this. Then I started to use it." She shared photos almost on a daily basis. When she traveled, the number of photos posted increased. She opened Instagram five or six times a day. She not only followed her friends, but also celebrities. "I enjoy watching private life of celebrities. I enjoy my friends' photos and check in which restaurants they had lunch/dinner. Also, I learn how to take a good photo." Nozomi also shared photos of food and interesting things she found in her everyday life and travels. She often shared photos of fancy food in restaurants/cafes, and also bento (lunch box in Japanese) and dishes she made.

Sometimes Nozomi did not share photos even though she had fancy food in a good restaurant. "When I share photos of fancy food on Instagram, I'd like to make my friends jealous. But I am careful not to do it too much." The photos of bento and dishes she made were taken and shared mainly for the record, but she "styled" the food so that it looked nice. She often enjoyed looking at the old photos on Instagram. “I can't imagine my life without Instagram. Good memories and my daily things are on it. It reminds me of what I have done with visual images." Nozomi's use of Instagram was dual functional - a tool for self-diarization while also performing the mundane 
for friends. While the images were of everyday situations like food, they were presented in a highly stylized way. Here Instagram allowed her to transform the mundane into a spectacle.

21-year-old graduate student, Rina, bought a sumaho when she started university. She used LINE, Twitter, Facebook, Instagram and Snapchat. For photo sharing, she used Instagram the most, which she started to use for its editing features. Rina used to use Facebook for photo sharing, but she got tired of it. "I'm full. There are too many friends on Facebook and I'm tired of watching photos of their life events." Her Instagram account was private, and Rina was trying to limit her followers to 100, so sometimes she declined requests from friends who were not close. As she noted:

Instagram is the most 'homely' social media for me. On each social media, my degrees of self-disclosure are different. For example, on Facebook $20 \%$, on Twitter $30 \%$ and on Instagram more than $70 \%$. About LINE and Snapchat, I use them to communicate with only familiar people.

Rina shared photos once a week, when she wanted to express something or to keep records. Recently she had enjoyed visiting beef barbecue restaurants and when she visited a new restaurant, she shared photos with location information. Here we see Rina using Instagram to package the mundane as informative so that her friends might learn of different restaurants. Through these snapshots of the mundane Rina highlighted difference and similarity between herself and her friends.

For our Tokyo participants, one of the biggest motivations for sharing photos was to attract their friends' interest and reinforce the significance of their co-present in everyday life. To accomplish this purpose, respondents carefully took, selected, edited and shared photos of their everyday life. Being both mundane and intimate in content and context, the pictures sought to create an ambience of being there. The participants cared about their friends' reaction to their photos. They checked the number of "Likes" and comments, and also talked about the photos when they met with friends. Those photos were very important tools for their "impression management," evocative of Goffman's (1959) work around presentations of self in everyday life. The collection of the photos on Instagram represented not only their everyday experiences but also how they wished to tell their everyday experiences and wanted to be seen (Holland 2015; van Dijck 2008).

Some of our participants connected with their sibling/s and/or wife/ husband, but none of them connected with their parent/s on Instagram. The "impression management" to be seen "cool" is directed towards mainly 
close friends and family members of the same generation. Here we see that through Instagram they created intimate publics that can differentiate between sharing with family and friends (Holland 2015; Vivienne and Burgess 2013). Many of the participants used Instagram in combination with social media LINE to compartmentalize what their parents see and don't see. Respondents used LINE, rather than Instagram, to share photos with their parent/s.

The collection of photos on Instagram was not only important for keeping in constant contact with friends but also for their self-satisfaction. Also, an act of selecting 'proper' photos for sharing can be understood as a reflection of one's desire to be recognized and approved by other members of the community (Van House 2011; Watkins et al. 2015). By carefully maintaining one's images to be shown/not shown, he/she manages to situate him/herself within the social relationships (Uimonen 2016; Litt and Hargittai 2014). When they got tired in everyday life, they recalled pleasant moments "to feel happy" by scrolling the feed on Instagram. And they rediscovered their friends' comments and the number and name list of the "Like" button. Once they shared photos on Instagram, the photos became something more meaningful in conjunction with their friends' reactions (van Dijck 2008; Sarvas and Frohlich 2011).

\section{Melbourne}

Smartphones with locative technologies are relatively pervasive in Australia, and this rate of pervasiveness is increasing rapidly. Smartphone penetration in Australia was $37 \%$ at the beginning of 2011 ; two years later, at the beginning of 2013, that figure had risen to 65\% (Our Mobile Planet 2013; ACMA 2013). But unlike Tokyo, which already has a decade of mobile internet, Melbourne is relatively new to the phenomenon. Other recent research has indicated that the number of people who own either a smartphone or a tablet has risen to over $70 \%$ in this Australian city (Deepend 2014). Device penetration is relatively high across most age demographics. Within certain age brackets, such as the $25^{-29}$ demographic, ownership of smart devices is over 9o\%; however, even older demographics, such as the over-6os, report smart phone ownership of $55 \%$ (Ibid.).

Unlike Japan's highly gendered use of Instagram, Australia has about $60 \%$ women and $70 \%$ of total users are between the ages of 18 and 34 . In recent statistics released on social media usage, Instagram has 5 million monthly active Australian users, which represents a $30 \%$ growth in the last 12 months and implies that around $21 \%$ of the Australian population own 
an Instagram account (Social Media News 2015). Australians follow these main interests: friends (61\%), photography (40\%) and travel (40\%). These dominant genres were echoed in our fieldwork in Melbourne. Just as the genres and demographics are similar to Tokyo, the way in which Instagram is used to rediscover the poetics of the mundane and the everyday can also be paralleled between the two sites.

22-year-old Yana lived in the outer suburbs of Melbourne with her partner 28 year-old Nathan and her young son. She was a keen user of Facebook, Instagram and Pinterest, but she only considered Facebook as being "social," and somewhere she would actively seek to have a conversation with her friends or have a look at what they were doing. She considered Instagram and Pinterest to be personal entertainment, where she chose to only browse images. As Yana described Instagram, "For me, it's not a social thing, it's just for me."

When we visited Yana in her home, it was clear that she lived in a family of collectors. Nathan had a cabinet full of Star Wars and Call of Duty figurines, models and Lego that he made and displayed, and her son uses the dining table as a space where he built his own Lego displays. "Collecting" gives us some further clues to how Yana thought about social media, as prior to using Pinterest and Instagram she was an avid subscriber of Fashion Trends Forecast magazines. Yana reflected:

I paid for forecasting magazines and they're not cheap and I think that this (Pinterest and Instagram) is more relevant than a forecasting magazine because a forecasting magazine is only really seen by people in the industry, whereas, this is seen by everybody and I think this is more valuable than a forecasting magazine just quietly.

Yana still kept her magazines stacked in piles in her study. "It hurts me to throw them away, so I've started recycling them and using them for things and chopping them up. For gift wrapping paper and stuff." Yana's study was connected to her kitchen by a long corridor and she had decorated the walls with collages of photos hung in frames. She printed the photos from her phone and digital cameras before she owned her smartphone. "They're all from the phone. Just family stuff, that's just fun that you don't really want on social media, but I've got one up there I'm in a bikini, that's not going on Facebook but I'm happy to have it in my house sort of thing." Yana also kept boxes of printed photos with her magazines in the study.

In addition to her photos and magazines, Yana also had a collection of recipe books in a cabinet in her kitchen. She would look up recipes 
online and save them, although she pointed out that she also still used recipe books as well as online sources to inspire her. Although Yana's use of Instagram and Pinterest was less of a diary than those of the examples of our participants from Tokyo, her use of these platforms was still highly personal as they represent private collections that she used similar to the way she previously related to print media (Thudt et al. 2016; Watkins et al. 2015).

Family meals and food preparation have always been an important part of Lily's home life. Before she was married and still lived at home with her parents, enjoying her mother's meals with her siblings was a part of their shared experiences growing up. During one of our research visits, Lily had a bookshelf in her kitchen where she kept scrapbooks, exercise books and display folders of recipes she had collected over the years, along with recipe books she had bought or had been given. As Lily reflected:

This is like my old folders; this is all my sweets, that's all my savories, so sweets a lot thicker, but I don't use all of these recipes so I was finding I really had to go through, and what I was planning to do was go through and either make notes or throw out the ones that I don't use. So, cheesecakes, I might have two recipes; if this one's better I want to keep one and chuck the other, but I've never gotten round to it. So there's heaps in here, and there's some I will keep coming back to and others I haven't used since I've printed them out. So they do need a good sort through.

Lily had downloaded the Paprika app on her phone, which allowed her to browse recipes and save them to her own lists, just like "lunches" or "baking." Lily reflects:

I do still like print copies, so I do have recipes books, I do have booklets of recipes as well. But Paprika is pretty good as well because it's all there and you can have your own categories, desserts, mains, whatever you want. You can tag them anyway and so it's sorted there.

Lily also took photos of the dishes she prepared, mostly baking and shared them over WhatsApp with a few of her friends, a couple who were also young mothers who she exchanged meal planning ideas with and another who enjoyed baking. "Generally, the finished product, but because it's been baking breads and scrolls and stuff then it could be in the middle when it's proving; 'How does this look? Is it almost there?' If someone just wants tips or something." She kept some of the photos if the dish looked 
exceptionally good or if it was a good photo, but she deleted most of the photos.

Collecting recipes and exchanging meal ideas with her friends also allowed her to bond with her mother. As Lily noted:

She's a good cook so she's got lots of good stuff that I enjoy, so these are the key ones and I'm like, "Can you please just write that down before we lose you" or whatever, you know what I mean. Because she cooks by feel she doesn't really have recipes, so she was trying to capture all that for me. So yes, there's a few but they're really old, you can see. Some are just her dictating, "This is what I'm doing" so I'm trying to scribble it down. And it's never accurate because she'll taste and she'll add a bit of this, a bit of that.

Recipe browsing and curating websites and apps had complimented her hard copy collection of recipes. Lily continued:

Everything now is so much easier on the net, so it's easier to sometimes go to your Safari and look up a recipe. Taste has heaps and there's good ones there too. The ones that I know are good I've started trying to put into Paprika, or ones that I want to try, and then over there it's easy, you can make notes as well. If you've tried it once and it's too salty maybe come down in salt, so I'll make a little note in there.

In Melbourne, taking and retaining images as archiving can be better understood in relation to habits of collecting prior to social media. In the cases of Yana and Lily presented below, collecting was experienced as a private past time. Before discovering Pinterest, Yana was an avid collector of magazines and Lily has an extensive collection of recipes, both in the forms of "old media" from hand-written notes from her mother and magazine clippings and "new media," where she has downloaded a couple of apps for collecting and curating recipes.

Further, food and food preparation were an important part of Lily's family and social life. When she had baked a dish for example, she would share a photo of the finished product with her friends over WhatsApp. Lily did not intend to keep or archive her images; rather, they were a type of visual conversation with her friends at that time (van Dijck 2008; Holland 2015). By contrast, Yana's husband Nathan was a professional chef. She often took photos of food he had prepared for the family, but she never displayed them digitally, but only showed them to her friends in conversation. 


\section{Shanghai}

Photo sharing is a common phenomenon on Chinese social media such as WeChat. The history of the camera phone practice can be dated back to the social networking website Qzone and different online photo albums in the 20oos. Unlike Melbourne and Tokyo, due to the Great Firewall (GFW, the Chinese governmental block practice for certain foreign websites, including Google, Facebook and Twitter), Chinese users have not had access to internationally popular photo sharing platforms like Instagram unless they used special ways to leap over the GFW. In the past decade, the popular platform for personal photo sharing has drifted from Qzone to Renren. com/ Kaixin.com to Weibo, paralleling the development of the internet in China. However, as WeChat got a huge number of monthly active users (549 million in 2015 Q1) (Tencent 2016), WeChat has gradually taken first place in personal photo sharing in China.

Within WeChat, users can not only take photos but also share them, either directly to certain friends or in WeChat Moments. The camera function built in WeChat Chats allows users to take and share photos at any given moment whilst in dialogue with others. The photo sharing function in Moments is designed as the basic social function. As pictures give more vivid information and draw more attention, the photo sharing function has successfully enhanced the social attribute of WeChat and has led to a new fashion within the Chinese social media arena.

Graduate student Tiffany moved to Shanghai from a southeastern coastal city called Xiamen. Being apart from her parents, Tiffany usually shared her life with her family via WeChat. She had created a family WeChat group that included her parents and herself. The family enjoyed sharing photos, especially photos of food, in the WeChat group.

In the screenshot above, Tiffany (on the right) shared a photo of dumplings from the school canteen to their family WeChat group at lunchtime, indicating the everyday event that she had had dumplings for lunch that day. Seeing the photo, her father replied with texts that he also had dumplings for lunch. This conversation over WeChat can be regarded an epitome of typical daily family communication on WeChat.

In addition to sharing photos of her life events with her parents, Tiffany enjoyed sharing photos in Moments. However, when she was doing photo sharing in Moments, she was cautious about the quality of the photo and the wording of illustration. "Photos you share speak for your taste and style. I want to be an interesting person and want to be regarded as an interesting one." 


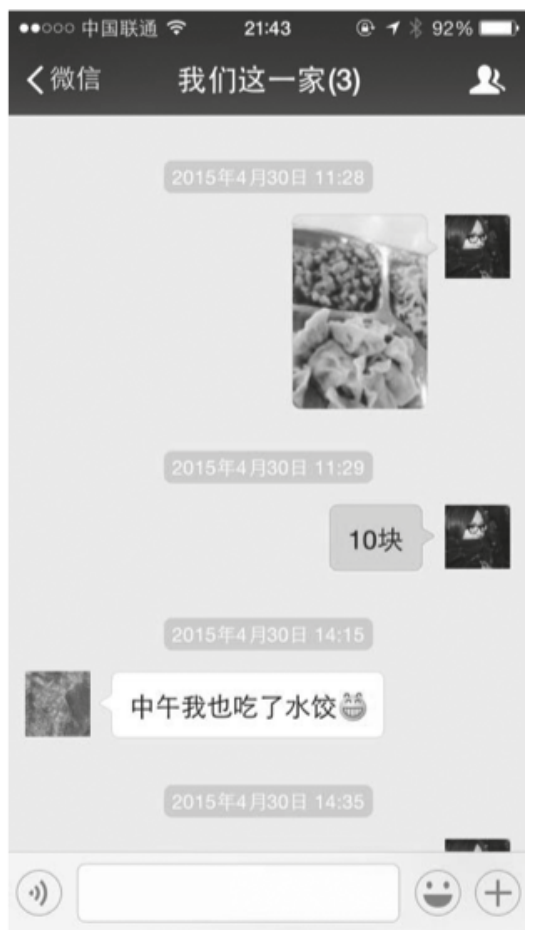

Figure 6.3: Tiffany sharing a photo of her school dumplings with her family

Tiffany posted nine photos after her trip from New York. She drew a parallel between photos of landmarks in NYC taken by her own mobile phone and landmarks on postcards. This fantastic idea won her a large number of "likes" on WeChat.

Ben was a graduate student of landscape design in Hannover, Germany. He was born and grew up in Shanghai. After finishing his undergraduate study in a university in Shanghai, he applied for a master's program in Germany to pursue his dream as a professional designer. Ben likes photography and was a guru in Photoshop.

Ben viewed the sharing of photos as a gendered preoccupation and did not share as many as his female counterparts. Instead, he shared photos occasionally. Notably, he would only share photoshopped photos of trips that he had been on, saying that. "When I go on a trip and I take some nice photos, I want to keep a record and to mark the unique experience. So sometimes I would share my photos in Moments when I am in that mood. Also, I feel better with processed photos instead of the original one." 
Wo ist Wochenende? Keine Ahnung. Immer unterwegs
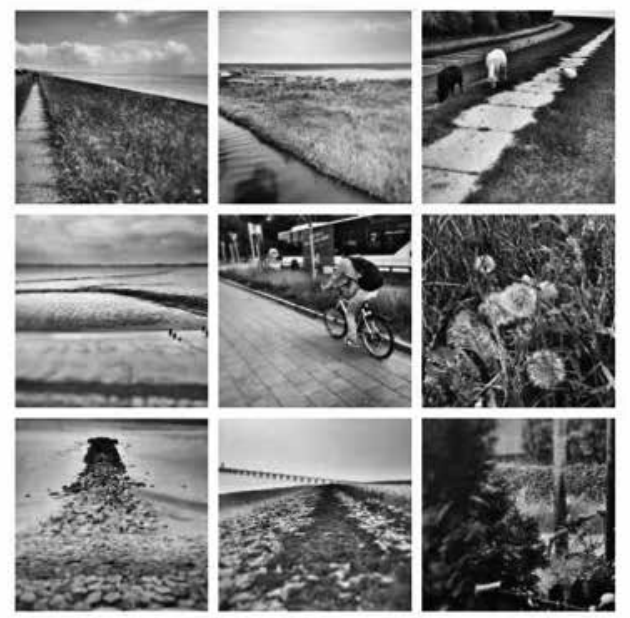

8 day(s) ago

Figure 6.4: Post by Ben

The role of photo sharing as an extended form of collecting continued to play an important part in each of the locations. Specifically, its role in managing relationships, co-presence and intimacy was significant. Participants spoke about having certain family or friends in mind when they posted something and using a variety of platforms to "select" specific contexts, meanings and audiences. In each of the multiple platforms of the participants, there were particular groups of intimates that then shaped what they shared and when they shared. The tension between digital archiving/ legacy/collections and ephemeral moments was apparent in the different usage from Snapchat to Instagram to WeChat.

As evidenced in the examples discussed in this chapter from three cities, younger generation users (mostly in the Tokyo and Shanghai cases) tended to use social media for displaying their day-to-day mundane, and as a form of "impression management." While they were careful enough not to make their friends "too jealous," the photos shared and the act of sharing itself were still playing an important role in displaying how they are organizing their everyday mundane experiences.

In part, cultural differences may become salient, with regard to the ways in which individuals present themselves to others. For these participants 
sharing photos was a form of communicating and expressing their tastes and lifestyles. In contrast, young mothers (in the Melbourne examples) were focused on the relationships within their family members and/or intimate friends and acquaintances. Rather than presenting tastes and lifestyles to a broader audience, their interests are geared toward sharing and nurturing memories within a confined group of people.

As demonstrated in this chapter, food is a popular subject to be captured and shared over SNS in the present cases. In fact, this tendency may be found across many other cultures as well. Whereas cases in Melbourne suggest that food photos are mainly for one's personal collection (also for practical reasons), cases in Tokyo and Shanghai illustrate that the SNSs play an important role as a site for demonstrating their eating and/or cooking behaviors. It also creates a site for constructing role images of housewives, or of female in general.

For example, in May 2015, the official Twitter account of the Cabinet Secretariat of Japan uploaded a photo of a bento box. There, a photo of kyaraben (a bento decorated to look like characters from anime) prepared for kids was shown, accompanied with texts depicting that the bento box is a symbol for a hard-working and caring mother. The page itself was titled as "For an encouragement of female (in Japan)" and was linked to a web page of a popular "charismatic" housewife who uploads kyaraben photos almost every day. Immediately after the tweet, the site was "flamed" with many complaints and oppositions to the idea of promoting such an image through bento photos.

The claims were that it was unrealistic for an ordinary working mother to spend that amount of time and energy on preparing bento boxes, and thus the image depicted was far from the "real" mundane social experience. This controversy shows how mundane social media photos can be taken out of context from a personal to a public realm. This instance highlights that while we might think we have control over the shaping of our intimate publics that are social mobile media, there are still many areas—especially in terms of digital legacy — that we are still to fully comprehend (van Dijck 2008; Holland 2015).

The ideas of sharing as a collective memory and non-sharing as a form of diarization may coexist balanced by both cross-cultural and culturally specific contexts (Uimonen 2016; Litt and Hargittai 2014). In part, such balance can be understood in terms of cultural differences, where users in Tokyo (and Shanghai) tend to use photos to present themselves for circulation purposes, with understandings that photos on social media may reflect socially desirable images of individuals. 
Occasionally, they acknowledge peer pressures to conform to certain tastes and lifestyles due to a relatively homogeneous culture. Still other parts can be understood in terms of participants' age (life stage) and their experiences with social media. Though there are cross-cultural differences, a focus on the act of collecting and sharing may be an entry point to speculate upon our images and understandings about role expectations (e.g. gender roles) within a society (Rose 2012; Holland 2015). In the next chapter we continue the focus on visual kinship to reflect how the visual genres play a role in how kinship is maintained, managed and mitigated.

\section{Conclusion: Sharing and Non-sharing}

Much of the literature into mobile visuality-especially in terms of camera phones - speaks about the important role of sharing as part of its logic (Frohlich et al. 2002; Kindberg et al. 2005; Van House et al. 2005; Koskinen 2007; van Dijck 2013). Indeed, as noted earlier, sharing has become a form of logic for contemporary cultural practice (Johns 2017). However, against this logic of sharing and co-presence emerge other, less articulated practices-most notably, the act of mobile visuality as a non-shared artefact. While previous research tends to pay attention to the social role of mobile media as a tool for communicating, socializing and accessing internet, we focus upon the internalized practices of mobile visuality as a non-shared activity that plays into different notions of memory making and cataloguing of the self.

As we found at the end of the study, many participants came to talk about non- sharing as a form of memory making. Against the compulsion of the digital archive that much of camera phone practices have contributed to, ephemeral apps like Snapchat and practices such as non-sharing (i.e. keeping the photo on the phone just for personal, individual use, started to emerge. This phenomenon seems to suggest participants are finding ways in which to resist the data trails and the default settings of locative media, which "emplace" place and time in the framing of the photo app geo-tag (Hjorth and Pink 2014). As algorithms and big data create anxieties around privacy, the option and right to not share will become more prevalent (boyd and Crawford 2011).

Moreover, with the rise in "spontaneous" and ephemeral media like Snapchat there is increasingly a need for researchers to think about mobile visuality beyond the archive. This non-sharing component of mobile visuality is about different forms of intimacy, memory and emplacement (Thudt et 
al. 2016; Uimonen 2016). In turn, this requires us to develop new methods that understand this non-sharing practice as part of nuanced reading of everyday life (Litt and Hargittai 2014). However, as we explore in the next chapter, sharing is still very much a way of curating co-present sociality and intimacy while emplacing locative media.

\section{References}

ACMA. 2013. "Communication Report." http://www.acma.gov.au/theACMA/Library/ Corporate-library/Corporate- publications/communications-report. Accessed 20 May 2015.

boyd danah, and Kate Crawford. 2012. "CRITICAL QUESTIONS FOR BIG DATA: Provocations for a cultural, technological, and scholarly phenomenon." Information, Communication and Society 15 (5): 662-679. DOI: 10.1080/1369118X.2012.678878.

Broekhuijsen, Mendel, Elise ven den Hoven, and Panos Markopoulos. 2017. "From PhotoWork to PhotoUse: Exploring personal digital photo activities." Behaviour and Information Technology 36 (7): 754-767. DOI: 10.1080/0144929X.2017.1288266.

Chalfen, Richard. 2011. "Doing family photography: The domestic, the public and the politics of sentiment." Visual Studies 26 (2): 176-178. DOI: 10.10 8o/1472586X.2011.571905.

Deepend. 2014. "Making digital work harder: Australian mobile device ownership and homeusage report 2014." http://www.deepend.com.au/download-whitepaper-device-usage-in-the-home. Accessed 20 May 2015.

Frohlich, David, Allan Kuchinsky, Celine Pering, Abbe Don, Steven Ariss. 2002. "Requirements of photoware." Proceedings of the 2002 ACM conference on Computer supported cooperative work, New Orleans.

Geismar, Haidy. 2015. "Post-photographic presences, or How to wear a digital cloak." Photographies 8 (3): 305-321. DOI: 10.1080/17540763.2015.110276o.

Goffman, Erving. 1959. The Presentation of Self in Everyday Life. New York: Random House.

Gómez Cruz, Edgar, and Asko Lehmuskallio. 2016. Digital photography and everyday life: Empirical studies on material visual practices. New York: Routledge.

Highmore, Ben. 2002. Everyday Life and Cultural Theory. London: Routledge.

Hjorth, Larissa, and Sarah Pink. 2014. "New visualities and the digital wayfarer: Reconceptualizing camera phone photography and locative media." Mobile Media \& Communication 2 (1): 40-57. DOI: 10.1177/2050157913505257.

Holland, Patricia. 2015. "'Sweet it is to scan...': personal photographs and popular photography." Photography: A Critical Introduction, edited by Liz Wells. 133-188. Abingdon; Oxon: Routledge. 
Hogan, Bernie. 2010. "The presentation of self in the age of social media: Distinguishing performances and exhibitions online." Bulletin of Science, Technology and Society 30 (6): 377-386. DOI: $10.1177 / 0270467610385893$.

Institute for Information and Communications Policy. 2014. "Heisei 25 nen jouhou tsushin media no riyou jikan to jouhou koudou ni kansuru chousa sokuhou (The results of a survey of the utilization time of telecommunication media and information behavior)." http://www.soumu.go.jp/iicp/chousakenkyu/ data/research/survey/telecom/201 4/h25mediariyou_1sokuhou.pdf. Accessed 30 June 2014.

Ito, Mizuko, Misa Matsuda, and Daisuke Okabe. eds. 2005. Personal, portable, pedestrian: Mobile phones in Japanese Life. Cambridge: The MIT Press.

Johns, N.A (2016) The Age of Sharing. New York: Wiley.

Jurgenson, Nathan. 2011. “The Faux-Vintage Photo.” The Society Pages. http:// thesocietypages.org/cyborgology/2011/05/14/the-faux-vintage-photo- full-essayparts-i-ii-and-iii/. Accessed 20 May 2015.

Kato, Fumitoshi, and Aiko Shimizu. 2005. "Moblogging as face-work: Sharing a "community-moblog" among project members." A position paper submitted to PICS Workshop (Pervasive Image Capture and Sharing: New Social Practices and Implications for Technology), UbiComp2005, September 11, Tokyo, Japan.

Kindberg, Tim, Mirjana Spasojevic, Rowanne Fleck, and Abigail Sellen. 2005. "The Ubiquitous Camera: An In-Depth Study of Camera Phone Use." IEEE Pervasive Computing 4 (2): 42-50. DOI: 10.1109/MPRV.2005.42.

Koskinen, Ilpo. 2007. "Managing banality in mobile multimedia." In The Social Construction and Usage of Communication Technologies: European and Asian Experiences, edited by Raul Pertierra, 48-6o. Singapore: Singapore University Press.

Kuhn, Annette. 2010. "Memory texts and memory work: Performances of memory in and with visual media." Memory Studies 3 (4): 298-313. DOI: 0.1177 / 1750698010370034 .

Licoppe, Christian. 2004 “'Connected' presence: The emergence of a new repertoire for managing social relationships in a changing communication technoscape." Environment and Planning Design: Society and Space 22 (1): 135-156.

Litt, Eden, and Eszter Hargittai. 2014. "Smile, snap, and share? A nuanced approach to privacy and online photo-sharing." Poetics 42: 1-21. DOI: 10.1016/ j.poetic.2013.10.00.2.

Lupton, Deborah. 2016. The Quantified Self: A Sociology ofSelf-Tracking. Cambridge: Polity Press.

Matsuda, Misa 2005. "Discourses of the keitai in Japan." Personal, Portable, Pedestrian: Mobile Phones in Japanese Life, edited by Ito, Mizuko, Daisuke Okabe, and Misa Matsuda. 19-39. Cambridge: The MIT Press. 
Mørk Petersen, Søren. 2009. "Common banality: The affective character of photo sharing, everyday life and produsage cultures." PhD diss., IT University of Copenhagen.

Our Mobile Planet. 2012. "Our Mobile Planet." http://www.thinkwithgoogle.com/ mobileplanet/en/. Accessed 21 May 2016.

Papailias, Penelope. 2016. "Witnessing in the age of the database: Viral memorials, affective publics, and the assemblage of mourning." Memory Studies 9 (4): 437-454. DOI: 10.1177/1750698015622058.

Pink, Sarah, Elisenda Ardèvol and Dèbora Lanzeni. 2016. Digital Materialities. London: Bloomsbury.

Pink, Sarah, and Vaike Fors. 2017a. "Being in a mediated world:Self-tracking and the mind-body-environment." Cultural Geographies. Online first. DOI: 10.1177/1474474016684127.

-2017b. Self-tracking and mobile media: New digital materialities." Mobile Media and Communication. Online first. DOI: 10.1177/2050157917695578.

Plummer, Kenneth. 1983. Documents of Life: An introduction to the problems and literature of a humanistic method. London; Boston: Allen \& Unwin.

-2001. Documents of life 2: An invitation to a critical humanism. London: Sage.

Reading, Anna. 2016. Gender and Memory in the Globital Age. Basingstoke: Palgrave.

Rose, Gillian. 2012. Doing family photography: The domestic, the public and the politics of sentiment. Kent, UK: Ashgate Publishing Ltd.

Sarvas, Risto, and David M. Frohlich. 2011. From snapshots to social media - The changing picture of domestic photography. London: Springer-Verlag.

Social Media News. 2016. "Social Media Statistics - August 2015." www.socialmedianews.com.au/social-media-statistics-australia-august-2015/. Accessed 21 May 2016.

Tencent. 2016. “2016 Annual Report.” http://www.tencent.com/zh-cn/content/ir/ rp/2016/attachments/201601.pdf (In Chinese). Accessed 1o February 2017.

Toyokeizai online. 2014. Toyokeizai online. http://toyokeizai.net/. Accessed 21 May 2016.

Thudt, Alice, Dominikus Baur, Samuel Huron, S., and Sheelagh Carpendale. 2016. "Visual Mementos: Reflecting Memories with Personal Data." IEEE Transactions on Visualization and Computer Graphics 22 (1): 369-378.

Uimonen, Paula. 2016. “I'm a picture girl.' Mobile photography in Tanzania.” In Digital photography and everyday life: Empirical studies on material visual practices, edited by Edgar Gómez Cruz and Asko Lehmuskallio, 19-34. New York: Routledge.

van Dijck, José. 2008. “Digital photography: communication, identity, memory.” Visual Communication 7 (1):57-76. DOI: 0.1177/1470357207084865.

—.2013. Culture of Connectivity. Oxford: Oxford University Press. 
Van House, Nancy A. 2011. "Personal photography, digital technologies and the uses of the visual." Visual Studies 26 (2):125-134. DOI: 10.1080/1472586X.2011.571888. Vivienne, Sonja, and Jean Burgess. 2013. "The remediation of the personal photograph and the politics of self-representation in digital storytelling." Journal of Material Culture 18 (3): 279-298. DOI: 10.1177/1359183513492080.

Watkins, Rebecca. D., Abigail Sellen, and Siân E. Lindley. 2015. "Digital collections and digital collecting practices." Proceedings of the 33rd Annual ACM Conference on Human Factors in Computing Systems, 3423-3432. 



\title{
7. Visual Generational Genres
}

\begin{abstract}
Chapter 7 considers the role of generational literacies and etiquettes around visual genres. For example in our study, younger participants tended to take and share more pictures, while older participants tended to take less but comment more on their children's images. Here, generational understandings of co-present gift giving rituals can be found.
\end{abstract}

Keywords: gift-giving; co-presence; visuality; food and travel genres

This chapter focuses on two popular genres of social media photographyfood and travel — and how families in Shanghai, Tokyo and Melbourne deploy them to partake in different forms of care at a distance (see Chapter 5 ). We consider genre as groupings of images as used by Horst and Miller $(2012,108)$, where "genre implies a combination of acceptability that is simultaneously moral, aesthetic and practical." Here the exploration of food and travel images, shared and circulated by family members are considered as part of maintaining digital kinship rhythms. Through these dominant intergenerational genres, we consider how cross-generational media literacies play out.

For example, in Japan, older participants tended not to share images as often as their younger counterparts, but they felt compelled to respond and comment on images posted by others as part of digital kinship to affirm and maintain family relationships. These older participants also felt that part of bonding with younger relatives was to acknowledge them through responding to their posts. On the other hand, younger users tended to upload more images on a regular basis, which was also found in Shanghai.

In our fieldwork, Shanghainese parents were also more likely to comment, illustrating some differences around expectations of obligation and responsiveness. And in the examples from Melbourne, sharing images of food and travel were common across wider age groups and were also reflective of lifestyle practices. In this circulation culture (as explored in Chapter 6) emergent practices_-such as non-sharing — are also playing new forms of

Hjorth, L., K. Ohashi, J. Sinanan, H. Horst, S. Pink, F. Kato, B. Zhou, Digital Media Practices in Households: Kinship through Data. Amsterdam: Amsterdam University Press, 2020 DOI 10.5117/9789462989504_CHO7 
intimate engagement. Across the three field sites, it was observable that circulating images, particularly around themes of food and travel contributed to how participants experienced family relationships.

Returning to Hochschild's (1979) notions of emotion work and feeling rules are especially helpful in considering how the circulation of images over social media platforms constitutes some of the work of the "family." That is, the kinds of labor that Erickson $(2005,338)$ describes as "activities that are concerned with the enhancement of others' emotional well-being and with the provision of emotional support." Significant to the study of emotions, gender and family life, Hochschild $(1979,561)$ has theorized emotion work as "the act of trying to change in degree or quality an emotion or feeling." She identifies how techniques of emotion work — such as changing ideas or thoughts, expressions or gestures - may alter a feeling that is experienced. Emotion work can be done or enacted by individuals directed towards others, enacted on individuals by others, or done towards oneself to change one's own state of emotions.

Feeling rules share some of the cues from other sorts of social rules, where what one should feel, when and for how long are deemed appropriate for a given situation. Some of the complexities around the appropriate expression of emotions are leveraged by affordances of social media such as "liking" or reacting on Facebook, for example. Hochschild (Ibid., 567) argues that as much as feeling rules have aspects in common with other norms of conduct, changes in feeling rules results in a lack of clarity of what an appropriate reaction should be. What emerged from our field sites was that between generations, there are different expectations as to the norms of what to disclose, what to withhold and the appropriate way to react to the posts of others.

As we discuss later in the chapter, some of our informants revealed the tremendous effort they invest into displaying and circulating images in a way that is sensitive to the expectations of others within their family. As aspects of digital kinship, images of food and travel and the responses they invite constantly acknowledge family relationships. Prior to social media, family meals and family travel have emphasized bonding and ideals of family life. Circulating images of food and travel holidays in particular, contribute to sharing the experience of eating or travelling, activities that ideally would be experienced together. These digital practices of sharing are an extension of the offline, and greatly contribute to a sense of intimacy, especially for transnational families.

Food photography and social media has been previously discussed in relation to aesthetics and consumption, and travel photography has been 
discussed in relation to family photography as memory making and performance of idealized family life (Rousseau 2012; McDonnell 2016; Chalfen 2011; Kuhn 2007; Rose 2012). In this chapter, we demonstrate how the making and circulation of food and travel photos over social media also have culturally specific inflections that emerge through comparison.

\section{Co-present Eating: Sharing Food Moments}

As one of the most dominant genres of camera phone practices, sharing images of meals is integral to maintaining co-present intimacy and digital kinship. Sharing a meal is an act of intimacy and cultural significance in many places (Counihan 1999; Tierney and Ohnuki-Tierney 2012). Take, for example, this opening image by Tetsuo, a 38 -year-old freelancer, who lived with his wife and three children in a suburban area, around an hour by train from the center of Tokyo. Tetsuo often worked from home and was quite active in caring for their children and undertaking daily chores-including cooking. As his wife was not particularly fond of cooking, Tetsuo usually prepared the family meals. Often, he shared images of the food he and the children ate together while his wife was still at work, a gesture that allowed her to feel a sense of co-presence.

Tetsuo also used his mobile phone to discuss the evening's dinner menu with his wife. Based on their conversation and her advice given, he then went grocery shopping for everything needed for the evening meal. For the participants living with other family members in the same household, sharing information around the "meal" was a daily routine. This included sharing photos of food in addition to the prepared dish; photos also included the ingredients bought, and dishes bought outside the home, such as in restaurants.

In Tokyo, regardless of household structures and family members, the most common topic uploaded and shared was around travel. For example, Rika, the flight attendant who lived in Tokyo who was introduced earlier, used LINE to video call her mother while she was in the United States, travelling in Sedona, Arizona. Even though Rika's mother lived very close to her, she lived alone, so Rika tried to video call her as much as possible. "We can talk face-to-face using LINE video call. And it's free. When I was in Sedona, I wanted to tell my mother, 'I am in a place like this!' by showing the scenery and my face." Rika's calls were enjoyable as much as they were reassuring to her mother. Along with Facebook, she found LINE the easiest mode of communication with her daughter, while she was away, "It's nice. I can feel 

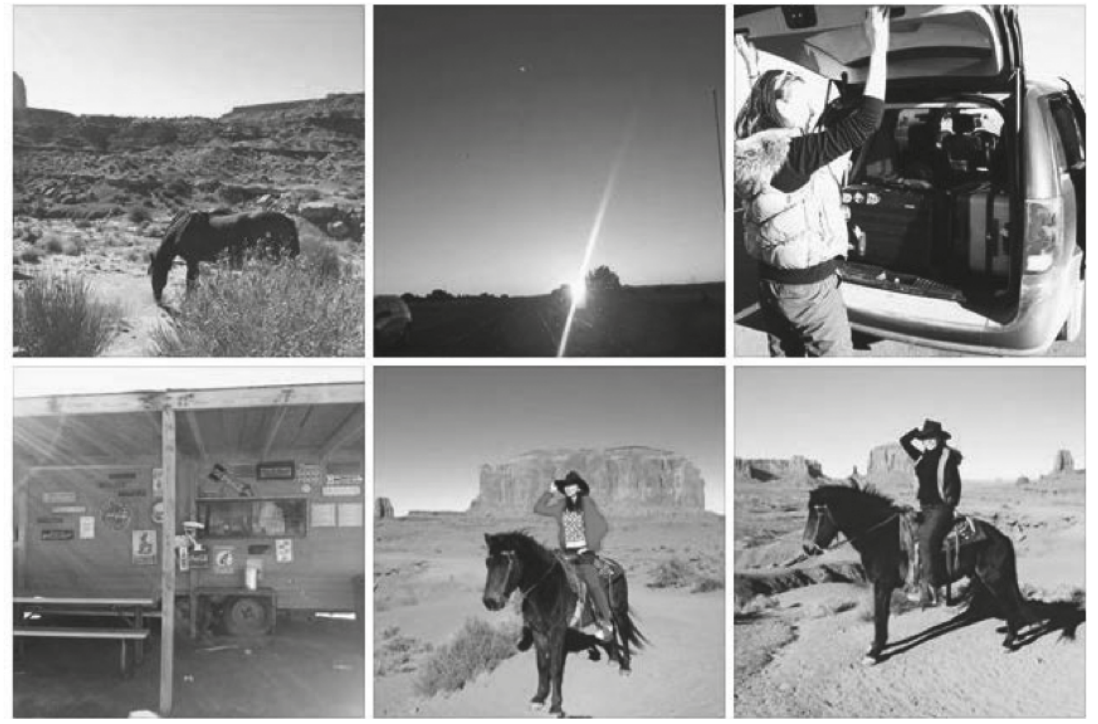

Figure 7.1: Rika's travel images

safe." Similar to the case of Rika, many participants explained the reason for sharing content related to their travels was that they wanted to share experiences they felt to be "extraordinary" with family members (Larsen 2005; Haldrup and Larsen 2003). They also wanted to keep other family members informed of their well-being. Travel photos operate effectively as a sense of friendly surveillance and the assurance of safety.

The majority of participants in Japan initiated contact with family members to coordinate or confirm schedules with one another via social media, rather than SMS. Take, for example, university student Shizuka who lived with her father, mother and younger sister in a condominium in the Tokyo area. Her busy schedule included studying, a part-time job, club activities, and catching up with her boyfriend. She often chatted with her family on LINE. She was not connected to her family on other social media platforms such as Instagram, Facebook and Twitter, but on LINE she communicated with them in a "family group." On her way home late at night, she would send a message to the family group on LINE to let them know what time to expect her. These kinds of gestures were reassuring to her mother, while her father stayed awake to let Shizuka in when she arrived home. Further, sharing updates around movements reassured family members of one's safety and assisted in coordinating schedules around meal planning.

Exchanging the image as a kind of information is as important as chatting for micro-coordination. Family members upload and share photos for the 
purpose of confirming safety, running errands, or coordinating calendars. At the same time, visualizing their day-to-day activities may serve to fulfill one's desire to be (socially) accepted.

Echoing Villi (2007), Gómez Cruz and Meyer (2012) who suggest that posting mundane photos of the everyday is not simply about showing mundaneness, but also the act of capturing and sharing the mundane is in itself stepping outside the mundane. The act of sharing a photo is also done in the hope that friends will see it on their timeline or newsfeed and will respond by "liking" the image. For example, travel and food photos are aesthetically pleasing and less confrontational forms of self- expression than sharing one's emotions.

The theme of "food-porn" has been observed in several contexts (McDonnell 2016). In Japan, there is an equivalent term meshi-tero where mesh $i$ is a meal, and tero is an abbreviated form of the word terrorism. Within close friendships, the term is used when friend(s) upload food photos, particularly during dinnertime or late at night. What makes these images meshi-tero is that they appear to be posted with the aim of appearing appetizing, while those viewing them are currently occupied with work or other tasks and are unable to leave to have a meal. Meshi-tero can be understood as a sign that the genre of "meal (food)" may have potential capacities to facilitate communication over social media in a routinized manner.

Although several of our participants in Melbourne were able to show instances of food photos posted by others on their Facebook or Instagram timelines, far less posted of images of food themselves. Yana, who appeared in the previous chapter, lived with her partner Nathan, who worked as a chef. Nathan considered himself a very private person, and although his cooking was outstanding - even when preparing meals for the family at home-he preferred not to display images of them to others. Yana also did not upload images of Nathan's cooking to Facebook, she says, "I just took pictures of it but this won't go on Facebook or anything like that but everyone at work has seen it but I won't post that because to me it's private." She preferred to show images on her phone to her friends at work where they could have a light- hearted chat about them, rather than "put them on display." Yana and Nathan illustrated a significant point about digital kinship and disclosure. By withholding images of Nathan's dishes, the intimacy of family space and family time was also maintained as private and not for the public display, consumption or gaze of others.

Circulating images of food and travel was much more important for others who have relatives and friends overseas. Stephen lived with his wife and teenage daughter. He had retired, but soon took up a part-time job in a fast 
food restaurant to keep himself busy. He moved to Melbourne in 1989, but he still visited his relatives, ex-navy colleagues, and former school friends in Malaysia and Singapore around once a year. He also used WhatsApp to chat with his friends overseas and he was the administrator of two Facebook pages, one for his ex-navy friends and one for his extended family. Stephen posted photos to both Facebook groups and he encouraged his ex-navy friends to post images to their page as well. He explained, "They can post what they're doing, where they've been, if the family is going somewhere, celebrations, that sort of thing, where we can connect as a family and as a group."

Stephen usually had his smart phone when his family went to dinner with his sister's family. Because they have lived in Melbourne for over 25 years, they usually tried new restaurants, rather than going to ones they had been frequented over the years. Although not a special occasion per se, Stephen would usually take a photo, particularly when the families were trying a new cuisine, and shared them on his profile page. Before owning the phone, he was using at the time of the research, Stephen only took family photos on holiday or visiting relatives overseas. Stephen posted both family outings and photos from within the home:

Family events, yeah, I do take photos, not many, just enough to remember the occasion ... Sometimes I post it on Facebook, but sometimes I just keep it in there (the phone). Sometimes I go outside and have a look at my plants I take a photo and post it. I want my family to have a look. I'm proud of my garden!

Several of the same groups of friends have visited Stephen and his family in Melbourne. Throughout these visits, Stephen was the main photographer and used his smartphone to take photos of his guests with his family at restaurants or at landmarks - especially if they were recognizably Australian-and shared them on his own profile, and also the ex-navy colleagues' page. When Stephen's family went on holiday, they would travel to Malaysia and other parts of South East Asia. Common holiday photos for Stephen and his friends were types of food, "like someone put they went to Korea, they had food they enjoy, this sort of thing. Especially when they travel." As well as keeping photos on his phone, Stephen backed them up to his desktop, a habit his wife was careful to ensure.

Drawing on John Urry's (2003) "tourist gaze," Haldrup and Larsen (2003, 25) explore family tourist photography through what they term the "family gaze." Employing a similar reflexivity as the tourist gaze, the family 
gaze draws attention to how sociality and social relations color the tourist perspective and photography. They suggest that the family gaze is "for acting out and framing active and tender family life for the camera. Family members and their performances make experiences and places extraordinary and full of enjoyable life" (Ibid.).

By approaching family photographic practices as a way of how families see the world and themselves, Haldrup and Larsen also emphasize that holiday photos do more than record keeping or documenting real experiences, they also "reveal more about the culture of imaginative families and idealized holidays than the people and places represented" (Ibid., 28). The family gaze takes a significant turn with mobile media discussed in relation to digital kinship. Family relationships shown through images posted more publically also creates ideals that are put on display, in the hopes that family members themselves may be able to live up to them.

As the administrator of his friends' page, Stephen also acted as content moderator. He encouraged family photos around food, travel and domestic life, but he discouraged his friends posting "rubbish" and "clutter," jokes that might be considered offensive, political opinions, and shares he saw as irrelevant. When they did appear, he removed these kinds of posts from the group. By encouraging photos of family life on the group page, Stephen also acknowledged the normativities around ideal family relationships as part of their shared identity as former colleagues.

A notable point from our case studies is that although posting photos was more typical for younger participants, posting travel and food photos was common across gender. Our observation challenges some of the assumptions around emotion work as well as family photography as being primarily the responsibility of women and mothers in particular. DeVault $(1999,56)$ draws attention to the different ways that women work to produce comfort within the family, from facilitating conversation about another family member's day, to preparing food as an expression caretaking.

Similarly, in the literature on family photography, mothers are emphasized as assuming the primary role in family photography making, curation and storage (Chalfen 1987; Rose 2003; Janning and Scalise 2015). These same authors also assert that as an extension of motherhood, mothers take responsibility for taking photos at key instances, organizing photos into albums and selecting and placing photos in frames to display in the home, where each of these acts serve to represent the family and idealized family life. Yet smartphones with high quality inbuilt cameras may represent a shift in the gendered nature of these practices, where digital kinship recognizes the efforts of maintaining relationships made by both men and women in the family. 

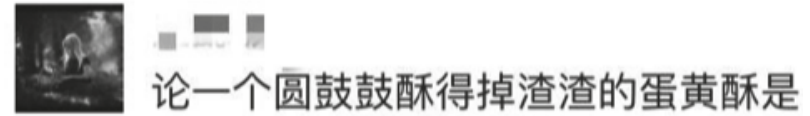
如何诞生的 @

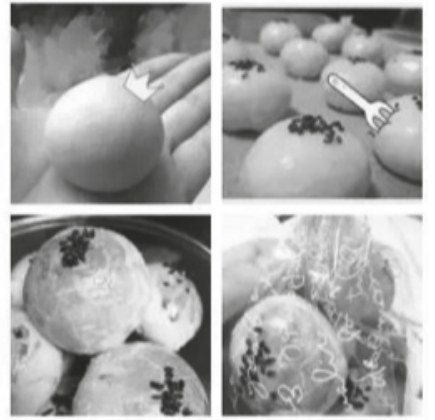

252 day(s) ago

Figure 7.2: WeChat moments, sharing food in Shanghai

In China, various genres of photos are circulated on WeChat. Food, travel and babies are among the most popular categories to appear as "Moments." Again, food and family meals are culturally important, and university students who participated in our research posted images of food regularly. 25-year-old Yi, for example, was a senior student at a university in Shanghai. She was particularly fond of sharing her homemade traditional Chinese dishes over digital media. Yi also considered herself to be quite shy, she did not share photos she considered too revealing of her personal life, and she only posted images once every two to three months; comparatively less often than Japanese and Australian participants. Yi explains, "I love cooking. I felt it meaningful to share something made by myself."

Figure 7.2 shows an image of Chinese snacks Yi prepared and then shared as WeChat "Moments." When Yi shared an image, she took several photos whilst preparing her dishes, which also enhanced her enjoyment of cooking. Every time Yi displayed a new post, her mother "liked" it, which demonstrated her support for her daughter's hobby. Sometimes, Yi also shared photos of her cooking in their family WeChat group.

Historically, food has also been a habitual way of greeting in Chinese society. On meeting, it is usual for familiars to ask: "Have you had your dinner yet?" as a daily greeting (similar to the way the weather is the subject of small talk for the British). Sharing photos of food with family 


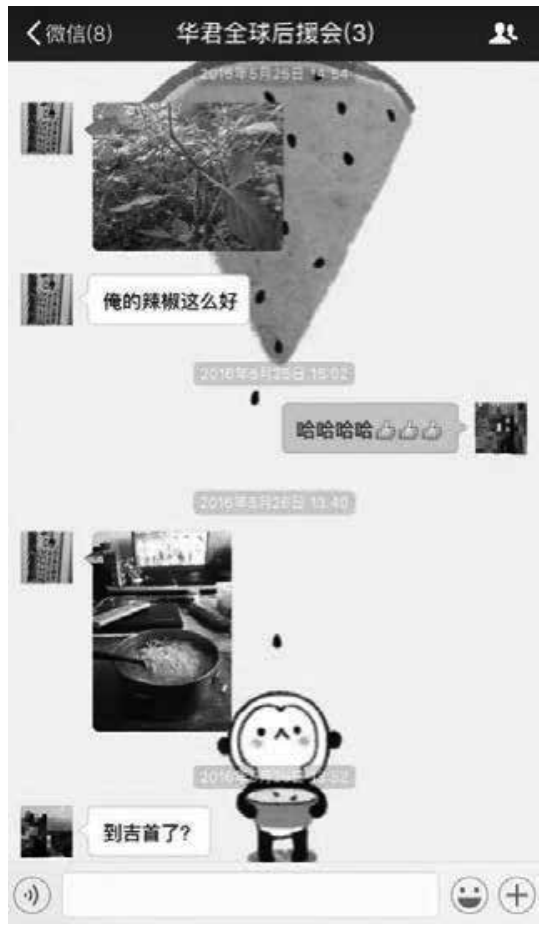

Figure 7.3: Chun's shared pictures

members is therefore a mundane practice on WeChat that reports the well-being of the person posting. Tiffany, who was introduced earlier, maintained a habit of sharing photos of food with families. "I did that just for fun, and to let them not be worried about my health." Another interviewee, 28-year-old student Chun, also shared a screenshot of her family sharing photos of food.

In this screenshot from Chun's phone, her father has shared two images. One is of his homegrown chili plant and the others show noodles he had cooked. By sharing these two photos, Chun's father conveyed two messages: firstly, that he had already arrived at home, and secondly, he was having a nice lunch. Notably in the second photo posted by Chun's father, he shared both the location and the dietary information with his wife and daughter, which is a typical example of mundane intimacy on digital media in the Chinese context. Images sent that reveal other useful information-such as the father's location and his dietary requirements - also reflect Miller et al.'s (2016) observation that due to social media, communication has become more visual. 


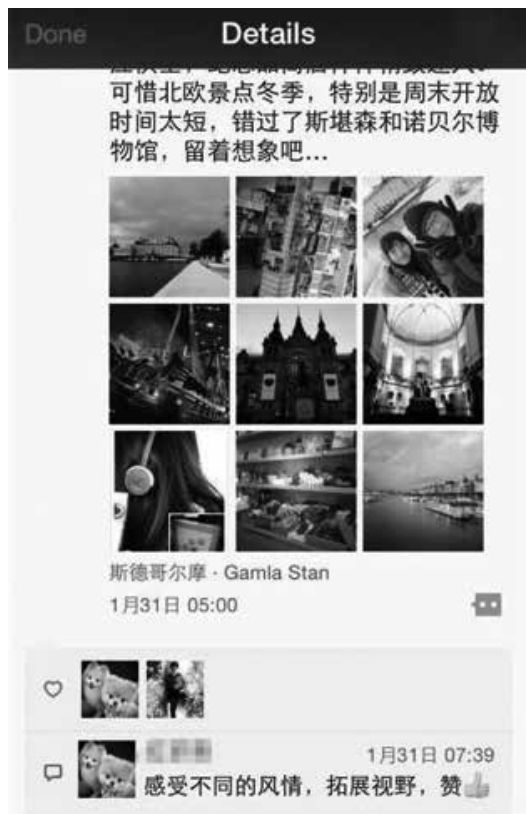

Figure 7.4: Jia's images on holiday

\section{Co-present Mobility: Sharing Travel Experiences}

According to participants who regularly share travel photos on WeChat, sharing photos of trips on social media was also a practice to commemorate life events. And by sharing travel photos in Moments, WeChat friends, including family members, could see the location as well as the "mood" of the person sharing, which can be read as an indication that the person is safe, especially when that person is quite young.

Jia, a 24-year-old young woman frequently shared travel photos. As a student who majored in tourism management, she liked travelling and enjoyed nature. During a trip to Europe, she posted updates to WeChat Moments almost every day.

Jia went on the European trip with her cousin Bo. The screenshot above shows several photos she posted of scenery in Stockholm. These images received the most "likes" from both her and Bo's mothers. Notably, Bo's mother reflected, "It was the first time for these two kids to travel abroad alone. To be honest, we've been worried about them." And Jia's mother described, "It is really convenient and comforting for us to find their travel photo in Moments, seeing them doing well and enjoying their time there." In addition to sharing travel photos in Moments, which is more public, 
some participants chose to directly share travel photos with their families in family groups on WeChat.

Qin and Jun had one daughter who was 25-years-old. Qin, Jun and their daughter interacted frequently on their family WeChat group. Their daughter shared a photo of a sunset during her trip with her parents in their family WeChat group. She had gone to Korea with two other friends and her parents felt relieved to receive her messages as well as seeing that she was enjoying her trip.

As we illustrate, families who live within close proximity and also for transnational families, the smartphone as a domestic technology plays a key role in how relationships are constituted and maintained, not only through pictorial depiction of the home and home life, but also through various uses of the smartphone within home life. These include organizing and coordinating daily tasks and routines, for capturing and sharing images of mundane and more eventful activities, and for research and entertainment. Hjorth $(2008,93)$ further asserts that the mobile phone is also "firmly embedded in what it means to experience place, co-present or not."

Doreen Massey's (2005) thoughts on locality have been influential for Hjorth and Pink, where she suggests a sense of locality is always mediated. For example, what constitutes "home" is mediated by memories infused with our sense of identity (Hjorth 2008, 94; Hjorth and Pink 2014). Place acts as "an organizing concept with fluid boundaries through which we can view and consider different configurations of online/offline combinations and the threads of sociality and visuality that traverse them" (Hjorth and Pink 2014, 46). By focusing on "emplacement" and co-presence, they also draw attention to where people, images, and technologies are always situated, in movement, and part of and constitutive of place (Ibid., 54). For transnational families, this is quite literal, where shared images are also, as Pink $(2011,7)$ describes, images that "are in movement as material or digital 'things' that travel from one locality to another."

\section{Conclusion}

This chapter has contextualized the exchange of images on social media, travel photos and food photos in particular within digital kinship as a mode of doing some of the work of the "family." Hochschild's (1979) notions of feeling rules and emotion work have been particularly helpful in relation to digital practices. Feeling rules as the norms and expectations around the appropriate ways to react, and when are first learned within 
the family (Thompson and Meyer 2007, 25). Images posted to social media act as cues from which the responses elicited display a range of emotions, from acknowledgement to relief.

As a form of "staying in touch," sharing photos can be compared to mutual gift exchange and generalized reciprocity, a theme that was explored in Chapter 4. And as discussed in Chapter 5, Baldassar (2007) identifies different kinds of care giving - from routine, the day-to-day form of care that is characterized by regular contact, and ritual care giving, the marking of special occasions and makes up "kinwork." For migrant families in particular, digital devices are used to maintain relationships in different ways including sending, sharing and displaying images, but also, as Baldassar, Baldock and Wilding (2006) argue, different forms of communication have different consequences for the family relationships involved.

One of those consequences is that more routine exchanges increase obligations and expectations, for more ritual exchange around significant events and for more visits. We have found that one of the ways family members navigate these expectations-either actual or perceived-is through withholding, a theme that is explored further in the next section of the book. Family members don't necessarily withhold communication, but they withhold what can be seen as "material evidence" of their lives and lifestyles "away."

Finally, Mason (1996) draws a distinction between the ideas of caring about, and the feelings and emotions of caring, and caring for as the actions, efforts and forms of labor that are invested in active caring. The circulation of travel and food photos are strongly resonant in relation to both caring about and caring for, which takes into account the family and family practices as represented in photos and in the acts of sharing and displaying them. Kinship through digital media practices also highlights the tremendous efforts that individuals invest into maintaining family relationships, whether in the same locality through forms of micro-coordination or through showing care at a distance.

\section{References}

Baldassar, Loretta, Cora Baldock, and Raelene Wilding. 2006. Families caring across borders: Migration, aging and transnational caregiving. New York: Springer. _.2007. "Transnational families and the provision of moral and emotional support: The relationship between truth and distance." Identities: Global Studies in Culture and Power 14 (4): 385-409. DOI: 10.1080/10702890701578423. 
Chalfen, Richard. 1987. Snapshot versions of life. Wisconsin: University of Wisconsin Press.

Chalfen, Richard. 2011. "Doing family photography: The domestic, the public and the politics of sentiment." Visual Studies 26 (2): 176-178. DOI: 10.1080/1472586X.2011.571905.

Counihan, Carole M. 1999. The anthropology offood and body: Gender, meaning, and power. New York: Routledge.

DeVault, Marjorie L. 1999. “Comfort and struggle: Emotion work in family life." The Annals of the American Academy of Political and Social Science, 561 (1): 52-63.

Erickson, Rebecca J. 2005. "Why emotion work matters: sex, gender, and the division of household labor." Journal of Marriage and Family 67 (2): 337-351. DOI: 10.1111/j.0022-2445.2005.00120.x. Accessed 10 March 2018.

Gómez Cruz, Edgar, and Eric T. Meyer. 2012. "Creation and control in the photographic process: iPhones and the emerging fifth moment of photography." Photographies 5 (2): 203-221. DOI: 10.1080/17540763.2012.702123.

Haldrup, Michael, and Jonas Larsen. 2003. "The family gaze." Tourist Studies 3 (1): 23-46. DOI: 10.1177/1468797603040529.

Hjorth, Larissa. 2008. "Being real in the mobile reel: A case study on convergent mobile media as domesticated new media in Seoul, South Korea." Convergence 14 (1): 91-104. DOI: 10.1177/1354856507084421.

Hjorth, Larissa, and Sarah Pink. 2014. "New visualities and the digital wayfarer: Reconceptualizing camera phone photography and locative media." Mobile Media \& Communication 2 (1): 40-57. DOI: 10.1177/2050157913505257.

Hochschild, Arlie R. 1979. "Emotion work, feeling rules, and social structure." American Journal of sociology 85 (3):551-575. DOI: 10.1086/227049. Accessed 2 March 2018.

Horst, Heather, and Daniel Miller. 2012. "Normativity and materiality: A view from digital anthropology." Media International Australia 145 (1): 103-111.

Janning, Michelle, and Helen Scalise. 2015. "Gender and generation in the home curation of family photography." Journal of Family Issues 36 (12): 1702-1725. DOI: 10.1177/0192513X13500964.

Kuhn, Annette. 2007. "Photography and cultural memory: a methodological exploration." Visual Studies 22 (3): 283-292. DOI: 10.1080/14725860701657175.

Larsen, Jonas. 2005. "Families seen sightseeing: Performativity of tourist photography." Space and Culture 8 (4): 416-434. DOI: 10.1177/1206331205279354.

McDonnell, Erin M., 2016. "Food Porn: The conspicuous consumption of food in the age of digital reproduction". In Food, Media and Contemporary Culture: The Edible Image, edited by Peri Bradley, 239-265. New York: Springer.

Mason, Jennifer. 1996. "Gender, care and sensibility in family and kin relationships." Sex, Sensibility and the Gendered Body, edited by Lisa Adkins, and Janet Holland, 15-36. London: Palgrave Macmillan. 
Massey, Doreen. 2005. For Space. London: Sage.

Miller, Daniel, Elisabetta Costa, Nell Haynes, Tom McDonald, Razvan Nicolescu, Jolynna Sinanan, Juliano Spyer, Shriram Venkatraman, and Xinyuan Wang. 2016. How the world changed social media. London: UCL Press.

Pink, Sarah. 2011. "Sensory digital photography: Re-thinking 'moving' and the image." Visual Studies 26 (1): 4-13. DOI: 10.1080/1472586X.2011.548484.

Rose, Gillian. 2003. "Family photographs and domestic spacings: A case study." Transactions of the institute of British Geographers 28 (1): $5^{-18}$. DOI: 10.1111/1475-5661.00074.

-2012. Doing family photography: The domestic, the public and the politics of sentiment. Kent, UK: Ashgate Publishing Ltd.

Rousseau, Signe. 2012. Food and social media: You are what you tweet. Lanham: Altamira Press/Rowman \& Littlefield.

Thompson, Ross A., and Sara Meyer. 2007. "Socialization of emotion regulation in the family." In Handbook of Emotion Regulation, edited by James Gross, 249-268. New York, London: The Guilford Press.

Tierney, R. Kenji, and Emiko Ohnuki-Tierney. 2012. "Anthropology of food." In The Oxford Handbook of Food History, edited by Jeffrey M. Pilcher, 117-134. Oxford: Oxford University Press.

Urry, John. 2003. Global Complexity. Cambridge. UK: Polity Press

Villi, Mikko. 2007. "Mobile visual communication: Photo messages and camera phone photography." Nordicom Review 28 (1): 49-62. 


\section{Section IV}

Co-futuring Kinship 



\title{
8. Re-imagining Digital Care and Health
}

\begin{abstract}
In Chapter 8 we turn to Co-futuring Kinship — the ways in which past and present practices inform how the future of the kinship for care at a distance. This is particularly important for "super-aging" contexts like Japan in which one in three is of 80 years old. Chapter 8 sets the picture for discussion around digital health in which mobile media is fully imbricated in. Discussions around a "silver bullet" in the form of a mobile app still dominate despite the fact that there is much work into the need for social, rather than technological, solutions.
\end{abstract}

Keywords: aging; digital health; mobile apps

In this section of the book we explore the role of mobile social media practices as a vehicle for quotidian, mundane notions of digital health. Rather than conceiving digital health as something that is designed by technologists and biomedical scientists for others, we consider the important role of the lived experience of everyday users to create their own forms of health and wellbeing in, and through, digital contexts. As surgeon Atul Gawande notes, aging in Western contexts has often been simplified through a medical lens - instead, Gawande argues that aging needs to be understood as a more holistic social and cultural phenomenon, where it is about finding meaning and purpose throughout all of life's transitions (2014). Understanding aging as part of a continuum, in which intergenerational unidirectional sociality is constantly at play, is key to Co-futuring Kinship.

Indeed, Co-futuring Kinship requires us to reflect upon the ways in which the digital creates and affords different forms of care, continuity and connection. We deploy the concept of co-futuring as a way to emphasize our participants' role in designing the contextual uses of digital media in everyday life. Co-futuring, as defined by UNESCO chair Sohail Inayatullah, is a design process that allows participants to relate differently to the past and present (2008). Inayatullah defines six different ways to think about

Hjorth, L., K. Ohashi, J. Sinanan, H. Horst, S. Pink, F. Kato, B. Zhou, Digital Media Practices in Households: Kinship through Data. Amsterdam: Amsterdam University Press, 2020 DOI 10.5117/9789462989504_CHO8 
co-futuring as a way to collaborate with different stakeholders to investigate future solutions for sustainability and social inclusion. Co-futuring sees participants as co-designers in their futures as informed by their past and present practices. Co-futuring puts lived experience at the center of the participatory process. In the case of growing datafication of life in which citizens can feel disempowered by everyday corporate or governmental surveillance, co-futuring allows for more micro and more intuitive forms of activism.

The notion of co-futuring became prevalent in our ethnography over the three years. Over that time, relationships and digital media dynamics changed and evolved. Elements such as tracking and automation became heightened in conversations and practices. As datafication became increasingly prevalent through mobile devices in everyday life_-from intentional self-tracking to more un-intentional forms of surveillance by corporations and governments - we needed ways in which to not only reflect on the impact of these technologies but also the implications on our future lives. For example, what happens to our data when we die? Many of our participants were only starting to think about this and many didn't have digital legacy plans in their wills.

In this final section the book turns more to reflection on present practices as future-in-making-co-futuring. We returned to our participants once more to gain their insights into how digital media-especially in terms of locative and mobile media-were starting to take on new dimensions. In particular, we focused on aging and the role of mobile media for informal notions of health and care. We discussed with both our participants as well as including older adult family members about their relationships to health and care in an age of datafication. Were technologies helping to enhance certain aspects of health? Was the technology camouflaging bigger issues about intergenerational care?

We begin this chapter with a discussion of mobile games in relation to quotidian digital health. We then reflect upon the complex and often contradictory space of applification and datafication alongside hearing our participants' valuable insights. By connecting practice with co-futuring approaches we hope to reconnect lived experience with hopes and desires for a future in which technologies are embedded within the social.

\section{Mundane Mobile Games as Quotidian Digital Health}

We met with Machiko, an 86-year-old woman who lived in Kyoto. When her husband died a decade ago, Machiko started going to her local gym, 
as a way to make friends and focus on her health and wellbeing. While she made some good friends, over the past decade Machiko found that some of her friends had passed away, while others had increasing mobility issues. Machiko felt less motivated to go to the gym when her friends weren't there.

One day she went to the local park with her friend Koko and Koko's grandson, Haru. Haru asked Koko and Machiko if they could play with him. He guided them around the park showing them the digital overlays of the Pokémon GO universe. They had fun playing. Haru asked Koko if she might play it often and so they could play in parallel. Machiko offered to play with Koko to help. After a few months, Koko and Machiko started to feel that they understood the game and would often go for a walk to achieve steps for fitness whilst also playing Pokémon GO.

Since its highpoint in 2016, Pokémon $G O$ has begun to attract older players as a way to avoid social isolation and increase physical activity. In suburban areas of Kyoto, it is not uncommon to see players over the age of 60 slowly navigating their neighborhoods through the augmented Pokémon $G O$ interface. In a country with a large and growing aging population, Japan is now facing a future in which people will increasingly age and die alone. In places like Singapore, the elderly has also embraced Pokémon $G O$ as a way to keep fit and socialize, while also opening up opportunities to play and interact meaningfully with younger generations.

But Asia is not alone. In locations like Badalona, Spain, social workers are teaching older adults who live alone to play Pokémon $\mathrm{GO}$, and engage with others by joining local Pokémon GO communities of players (Hjorth and Jimenez 2019). Here, young and old play together in urban spaces. What becomes apparent is that there are ways of addressing various aging and health issues that are more effective than medication or new-fangled technological devices. Rather, through the now mundane and habitual medium of the mobile phone and a somewhat hackneyed three-year old mobile game - which is old for a mobile game - significant improvements to quality of life can be achieved. Pokémon $G O$ transforms urban space into something playful and social.

Ethnographic exploration of these lived experiences highlights the need for digital health to focus more carefully on the social dimensions of aging well rather than just having a "silver bullet" solution in the form of yet another mobile app. Instead, we can learn a lot from "old" media like Pokémon $G O$ and how they can be redeployed and reinvented in ways that demonstrate the importance of understanding social practice in designing for digital health solutions-especially in terms of aging well. 
Here Pokémon GO signals a few future directions for mobile games.

Firstly, mobile games afford both ambient and located social connection, and so can be used to counteract the emotional and physical isolation experienced by many people-young and old - in playful and innovative ways. Secondly, they can facilitate connections between younger and older generations through an interface that has mutual significance and enjoyment for both, creating the possibility for social change. Thirdly, they move across public and private spaces in ways that can usefully link informal and formal systems and routines of care, especially as we move towards aging societies.

Fourthly, mobile games also provide us with ways to understand the complex relationality between humans and our companion species, as we form intimate bonds through playful interaction, share sensory and affective modes of perception and proprioception, yet are distinct in our embodied uses of media. Fifthly, in an age of Big Data and gamification, and an associated focus on the quantitative and utilitarian aspects of technology, the sensory intimacy and ambient sociality of mobile games and playful apps force us to attend to the complex tapestry of media use, and the corresponding ethnographic and interpretive adaptability required of researchers.

The rise of Pokémon GO illustrates the importance of haptic digital media in affording space for the non-normalized body. Haptics allow players with impairments like hearing or vision to be able to participate in meaningful ways. The rise of digital media in terms of multisensorial dimensions such as haptics — which marries media and sensory study approaches—will be increasingly important as we move towards aging and super-aging societies (that is, over a third of the population is over 65 years). In the next section we contextualize this phenomenon in relation to debates around mobile applification and datafication. We then turn to the concept of feeling data. This discussion provides context for Chapter 9 in which we discuss participants' practices and perceptions.

\section{Applified and Datafied: Quantified Self and Digital Health}

Since 2010, the significance of Big Data, and the response to it through forms of the QS movement, has been unmistakable (Kennedy et al. 2016). As danah boyd and Kate Crawford (2011) note, much of the debates around Big Data need to acknowledge that no matter how "big" the data, it is always subjective. Data questions are riddled by the searchers' own perception and thus inherently human in their scope. Moreover, they argue the need for 
transparency, and access to the ways in which algorithms shape definitions of society. For ethnographers such as Dawn Nafus, big data, like little data, is about storytelling (Nafus 2016; Nafus and Neff 2016).

As digital health experts such as sociologist Deborah Lupton (2016) have noted, datafication has created a double edged sword for health-selftracking offers insight and a space for reflection about one's own exercise and yet, this same reflective data can be used by corporations in ways we are still only just understanding in light of, for example, the recent Cambridge Analytica debacle. For Natasha Dow Schüll, wearable technology allows for the designing of "self care" (2016) which has paradoxical dimensions. She argues:

key artifacts in a new cultural convergence of sensor technology and selfcare that I call 'data for life', wearables are marketed as digital compasses whose continuous tracking capacities and big-data analytics can help consumers navigate the field of everyday choice making and better control how their bites, sips, steps and minutes of sleep add up to affect their health. By offering consumers a way to simultaneously embrace and outsource the task of lifestyle management, I argue, such products at once exemplify and short-circuit cultural ideals for individual responsibility and self-regulation (cited in Ruckenstein and Schüll 2017, 317).

As aforementioned, concepts like applification and datafication have very much become part of quotidian urban everyday life with the saturation of smartphones. Datafication is the process whereby our personal data is given over to platforms for their own interests and financial benefit (van Dijck 2015). In the rise of datafication-i.e. where data saturates seemingly every aspect of our everyday lives - there have been two key camps. One camp stems from the QS movement founded by Wolf and Kelly in 2007 which sought to see individuals "gamify" their life in creative ways (Gilmore 2016; Lupton 2016b; Nafus and Sherman 2014) such as Strava and fit bit. In this space, approaches such as ethnography have blossomed to explore the multiple overlays of the digital in relation to the senses, sociality and movement through everyday places and spaces (Hjorth et al. 2017). This area explores what Jason Farman has called "social proprioception" (2011) — whereby the knowledge of the lived body and its movement is focused upon in the context of sociality. The other camp sees the darker side of neo-liberalism whereby data is being co-opted by corporations in ways that compromise privacy-what has been called dataveillance (Ruppert 2011; van Dijck 2015; Lupton 2016). 
Datafication and algorithmic cultures are now commonplace-that is, where data penetrate, invade and analyze our daily lives, causing anxiety and seen as potentially inaccurate statistical captures (Hjorth and Hinton 2019). These tensions and the paradoxes of power/powerless; tactic/strategy; identity/anonymity are highlighted through the lens of artists "playfully resisting" through creative projects that scrutinize the normalization ("bellcurving") of QS and datafication usage at a broader and everyday sociopolitical level. For some, self-tracking and QS allows for a better sense of the body - a deeper proprioception. Through thinking about the body as "laboratory," alternative human-centerd (and "more-than-human") social digital methods emerge around ethnography and critical making. But for others, the emphasis on numbers and quantification goes against real understanding of practice as something that is story-based and nuanced and can't be summed up by numbers (Lupton 2016; Humphreys 2018).

In general use applification is an IT industry term used to refer to one way of simplifying and making complex software and processes easier to use. Applification can be seen in the emergence of apps on the mobile device (van Dijck 2017). Applification, as part of "listening devices," raise important questions around privacy and surveillance and the use of the data by government, corporations and individuals. And while QS advocates will argue that mobile apps allow for the democratizing of data and tools for cartography and tracking, there are counter arguments about the deployment of data in ways we are yet to fully realize. This is especially in the case when user's die and they don't have a digital legacy provision in their will — thus affording corporations like Facebook to own their data, much to the horror of bereaved loved ones.

On mobile devices, apps are the embodiment of the ideal of applification. Fitness tracking apps like Strava (used mainly for bicycle riding and running) are indicative of how self-tracking mobile apps (as part of the broader QS movement) are playing a powerful role in people's lives. In these apps, one user's exercise becomes part of others' movements, allowing the data overlays about place-making to be shared as a social act. As users become more playful with their use of data and digital media, the role of feeling data, through complex multisensorial ways of being in the world becomes heightened.

\section{Feeling data}

The rise of self-tracking QS measures on mobile media allow for reflection-what some scholars have called feeling the data (Lupton 2017). Lupton has been working in the space of digital health for over a decade now. More 
recently her work has explored the role of what digital health "feels" like (2017). As Lupton notes, feeling the data can be understood as a process in which movement, cartography, data and place-making coalesce into a heighten sense of social proprioception.

This discussion raises one of the key tropes in current game studies around what a game "feels" like. Game designers such as Swick have highlighted that unpacking the often-tacit notion of the game "feel" is crucial to understanding player engagement (2008). Game phenomenologists such as Keogh have explored the role of game feel as part of the proprioception of the knowing, playing body (2018). Indeed, as important as "feeling" might be to the game or medium's affect, it has often been untheorized. Understanding and articulating the feelings associated around media and data requires complex multisensorial methods to unpack these experiences.

As Lupton identifies, more work into understanding the multisensorial dimensions of spaces and places is needed - in sum, the affective atmospheres (2017). As a term developed in cultural geography by Gernot Böhme (and especially mobility studies), it explores the affective and emotional attunements of humans and more-than-humans as they move through spaces and places. In areas like phenomenological psychopathology it is being deployed to understand the importance of atmospheres in helping with client consultation and related treatment (Costa et al. 2014). Focusing on affective atmosphere puts the lived experience at the center. As Lupton notes:

Affective atmosphere is understood as an assemblage of affect, humans
and nonhumans that is constantly changing as new actors enter and leave
spaces and places. Affective atmospheres are shaped by their multisensory
properties: how spaces and places are physically encountered via their
visual, haptic, aural, olfactory and taste properties is central to the feelings
they generate. Affective atmospheres can have profound effects on the ways
in which people think and feel about and sense the spaces they inhabit
and through which they move and the other actors in those spaces $(2017,1)$.

As Lupton argues, there is very little research into the "concept of affective atmospheres to the context of health, illness and healthcare" $(2017,2)$. With the rise of haptic screens through smartphones, the role of the digital as an embodied part of the everyday life comes to the forefront. Haptic media studies coalesce key areas: sensory studies, new materialism and new qualitative non-media centric approaches. It highlights the need to develop language around multisensorial approaches in and around haptics which emphasise the importance of the lived body and social proprioception. 
The rise of haptic media and trying to understand the phenomenon through practice has led to many subareas of ethnography including multisensorial ethnography (Pink 2009), tactile ethnography (Pink et al. 2016) and haptic ethnography (Richardson and Hjorth 2017). In particular, haptic ethnography seeks to coalesce the work done around mobile media in terms of the material, sensory and corporeal aspects (Richardson and Hjorth 2017). This process requires deploying methods and techniques that give voice to experience through multisensorial elicitation.

As Richardson and Hjorth note, haptic technologies such as smartphone screens are impacting our sensorial experience of being-in-the-world, beingwith-others and being-with-media (2019). Informed by and informing this approach, many researchers are increasingly focusing on the intimate, social and playful nature of mobile touchscreens. The role of haptic technologies is especially important when it comes to vulnerable agencies - that is, elderly, disabled or more-than-human users. Haptic technologies allow for multisensorial approaches to media that encourage different lived experiences and skillsets. Haptic screens re-focus our attunement away from the primacy of the visual and aural towards other sensorial knowledges. In this way, haptic screens require us to develop different techniques and methods in our ways of understanding the world.

At the intersection of the QS movement and gamification (Deterding et al. 2011), mobile media and its attendant apps are providing new ways in which to think about creativity, play and labor in everyday life (Hjorth 2018). From body measuring devices such as fitbits and apps to measure sleep, one could argue that everyday life is being colonized by datafication. However, in practice we see a diversity of resistance and subversives that suggest that not all of life is quantifiable (Neff and Nafus 2016; Hjorth 2018). We see how existing sociocultural practices and the ways in which users can queer the applification of everyday life through play and creativity. Indeed, far from just a medium for beautiful and young people, Instagram, like its audiences, are diversifying. Audiences are getting older and alternative, non-normative modes of body and performativity are being celebrated (Olszanowski 2014; Tiidenberg 2014).

\section{Conclusion}

In the next chapter we explore the complex and alternative ways in which the data can be felt by participants in quotidian contexts. We explore the practices and perceptions around data and caring intergenerationally. How does mobile and locative media provide particular forms of care? What 
problems around care does it highlight? Understanding the rise of mobile apps and self-tracking has become a key area of emerging research (Fox and Duggan 2013; Lupton 2016, 2017; Fox 2017). We begin with Japan as an example of a super-aging society which is grappling with its own cultural understandings of intergenerational care and digital health, then turn to our participants to explore their own experiences and reflections. We reflect upon rituals and perception as a process of co-futuring present practices.

\section{References}

boyd, danah, and Crawford, Kate. 2011. "Six Provocations for Big Data." In A Decade in Internet Time: Symposium on the Dynamics of the Internet and Society, September. https://papers.ssrn.com/sol3/pape"s.cfm?abstract_id=1926431. Accessed 10 February 2018.

Costa Cristina, Carmenates, Sergio, Madeira, Luís, and Giovanni Stanghellini. 2014. "Phenomenology of atmospheres. The felt meanings of clinical encounters." Journal of Psychopathology 20: 351-357. https://www.jpsychopathol.it/issues/2014/ vol20-4/SOPSI4-14.pdf\#page=12. Accessed 5 April 2018.

Fox, Nick. 2017. "Personal health technologies, micropolitics and resistance: a new materialist analysis." Health 21 (2): 136-153. DOI: 10.1177/1363459315590248.

Fox, Susannah, and Duggan, Maeve. 2013. "Tracking for Health," Pew Research Center: Internet and Technology, 28 January. https://www.pewinternet.org/ 2013/01/28/tracking-for-health/. Accessed 4 March 2018.

Gawande, Atul. 2014. Being Mortal. New York: Metropolitan Books.

Hjorth, Larissa. 2018. "Ambient and soft play: Play, labour and the digital in everyday life." European Journal of Cultural Studies 21 (1):3-12. DOI: 10.1177/1367549417705606. Hjorth, Larissa, and Sam Hinton. 2019. Understanding Social Media. Second Edition. London: Sage.

Humphreys, Lee. 2018. The Qualified Self: Social Media and the Accounting of Everyday Life. Cambridge, MA: The MIT Press.

Inayatullah, Sohail. 2008. "Six pillars: futures thinking for transforming." Foresight 10 (1): 4-21. DOI: 10.1108/14636680810855991.

Kennedy, Helen, Thomas Poell, and José van Dijck. 2015. "Introduction: data and agency." Big Data \& Society 2. DOI: 10.1177/2053951715621569.

Lupton, Deborah. 2016a. "The diverse domains of quantified selves: selftracking modes and dataveillance." Economy and Society 45 (1): 101-122. DOI: 10.1080/03085147.2016.1143726.

—.2016b. The quantified self: A sociology of self-tracking. Cambridge; Malden, MA: Polity Press. 
-2017a. "Feeling your data: Touch and making sense of personal digital data." New Media \& Society 19 (10): 1599-1614. DOI: 10.1177/1461444817717515.

_.2017b. "How does health feel? Towards research on the affective atmospheres of digital health." Digital Health 3. DOI: 10.1177/2055207617701276.

Nafus Dawn. 2016. Quantified:Biosensing Technologies in Everyday Life. Cambridge, MA: The MIT Press.

Nafus, Dawn, and Gina Neff. 2016. Self-Tracking. Cambridge, MA: The MIT Press.

Nafus, Dawn, and Jamie Sherman. 2014. "This one does not go up to 11: The Quantified Self Movement as an Alternative Big Data Practice." International Journal of Communication 8 (1): 1791-1793.

Olszanowski, Magdalena. 2014. "Feminist Self-Imaging and Instagram: Tactics of Circumventing Sensorship." Visual Communication Quarterly 21 (2): 83-95. DOI: $0.1080 / 15551393.2014 .928154$.

Pink, Sarah. 2009. Doing Sensory Ethnography. London: SAGE Publications.

Pink, Sarah, Jolynna Sinanan, Larissa Hjorth, and Heather Horst. 2016. "Tactile Digital Ethnography: Researching Mobile Media through the Hand." Mobile Media \& Communication 4 (2): 237-251. DOI: 10.1177/2050157915619958.

Pink Sarah and Vaike Fors. 2017. "Being in a mediated world: self-tracking and the mind-body-environment." Cultural Geography 24 (3): 375-388. DOI: 10.1177/1474474016684127.

Ruppert Evelyn. 2011. "Population objects: Interpassive subjects." Sociology 45: 218-233. DOI: 10.1177/0038038510394027.

Schüll, Natasha Dow. 2016. "Data for life: wearable technology and the design of self-care.” BioSocieties 11 (3): 317-333. DOI: 10.1057/biosoc.2015.47.

—.2017. Our metrics, ourselves. Public Books, 26 January. https://www.publicbooks.org/our-metrics-ourselves/. Accessed 2 March 2018.

Sharon, Tamar. 2017. "Self-tracking for health and the Quantified Self: re-articulating autonomy, solidarity, and authenticity in an age of personalized healthcare.” Philosophy \& Technology 30 (1): 93-121. DOI: 10.1007/s13347-016-0215-5.

Richardson, Ingrid, and Larissa Hjorth. 2019. "Haptic play: Rethinking media cultures and practices." Convergence 25 (1):3-5. DOI: 10.1177/1354856518815275. _.2017. "Mobile media, domestic play and haptic ethnography." New Media \& Society 19 (10): 1653-1667. DOI: 10.1177/1461444817717516.

Ruckenstein, Minna, and Natasha Dow Schüll, 2017. "The Datafication of Health.” Annual Review of Anthropology 46: 261-278. DOI: o.1146/annurevanthro-102116-041244.

van Dijck, José. 2014. "Datafication, dataism and dataveillance: big data between scientific paradigm and ideology." Surveillance \& Society 12: 197-208. 


\title{
9. Quotidian Care at a Distance
}

\begin{abstract}
Chapter 9 explores how some of these practices are playing out for our participants and how this informs generational imaginaries around data for care at a distance. How can we map and learn from some of the informal media methods for care at a distance around supporting older adult's independence and social inclusion? And how can understanding intergenerational emerging practices provide insight to that complicate definitions of datafication and dataveillance (using data to watch people)?
\end{abstract}

Keywords: informal care; care at a distance; social inclusion; aging

In this chapter, we focus on participants' practices and perceptions in and around Co-futuring Kinship through the idea of quotidian care at a distance. Co-futuring requires us to navigate the ways in which lived experiences are narrated in and through processes of the past, present and future. Understanding this complex and dynamic narrative requires us to acknowledge the ways in which intergenerational perspectives of care create multiple ways of being in the world. How does the data we make today impact upon how we reflect upon future notions of self? How can we make careful data? How are people starting to think about what data trails they will leave for their loved ones when they die? Aspects of Co-futuring Kinship recognize that the stories that we make of the world are a combination of experiences of the past and present projected onto feelings of the future.

We begin with Japan, deemed a super-aging country - that is, one quarter of the population are over 65 years old (OCED 2019). Many countries are turning to locations such as Japan to see how they address super-aging. What can we learn from Japan? What lessons are transferrable? What are culturally-specific? While images of robots or AI in aged homes dominate press media, the everyday Japan is much more mundane in its deployment of methods for care at a distance. We then turn to fieldwork in Shanghai and Melbourne to further reflect upon the cultural differences and parallels.

Hjorth, L., K. Ohashi, J. Sinanan, H. Horst, S. Pink, F. Kato, B. Zhou, Digital Media Practices in Households: Kinship through Data. Amsterdam: Amsterdam University Press, 2020 DOI 10.5117/9789462989504_CHo9 
There are many particular features to the Japanese super-aging context which are culturally, technologically and spiritually specific. For example, in rural Japan in which dying towns are growing, the deployment of automated cars to drop off groceries to older adults in remote mountain areas is becoming reality. Risks of older adults driving recklessly were heightened in April 2019 with the accident involving an 87-year-old driver, who ran off road out of control and killed a mother and child, as well as injuring 10 others (Inagaki 2019). With over 50\% of road fatalities caused by elderly drivers, there is a mounting case for driverless cars in Japan. Companies like Toyota are working with researchers and older adults to explore perceptions and practices given that much of the early work into driverless cars has been in urban areas. Issues such as network coverage and low consumer acceptance are but a few of the concerns.

In Japan, the average life expectancy is 87 for females and 81 years old for males (Ministry of Health, Labour and Welfare of Japan 2018). Even though Japan leads the rest of the world in life expectancy, there is a gap between the average life expectancy and healthy life expectancy (WHO 2016). In Japan, healthy life expectancy is 75 for women and 72 for men. This means that there is a decade in which dependency and decline affect quality of life. In a study conducted over two decades with 6000 participants, Akiyama found that about $80 \%$ of the older adults start to decline in health in their mid-seventies and need some kind of care (2000).

One of the key issues for prolonging healthy life is the prevention of frailty (Iwasaki 2017). Iwasaki describes "frailty" as the state between good health and need for personalized care. As a concept it covers physical, mental and psychological and social issues. For Iwasaki, frailty can be resolved with appropriate intervention. For Iijima (2016), there is a need to understand frailty as having three key aspects- physical, mental and social.

One of the key issues regarding aging well is social inclusion. Here media like Pokémon $G O$ that encourage social cooperation as part of their game play can be playful ways to combat these issues (Hjorth and Jimenez 2019). In Japanese press media, koritsu-shi (solitary death) cases have received significant attention due to the increase in single-person households (Kanawaku 2018). Kanawaku explains that often kodoku-shi (lonely death) is conflated with koritsu-shi (solitary death). For Kiwaki et al. (2015) the rise of loneliness/ social isolation in older adults has grown out of lifestyle shifts in post WWII Japan, in which adult children no longer live with their older parents to help with informal forms of care. 


\section{Informal Care}

As mentioned in Chapter 1, Pols's notion of care at a distance is useful in conceptualizing the way in which intergenerational care and digital media are playing out (2012). While Pols spoke of formal contexts of care in the case of palliative care, we reflect upon the often unspoken and mundane aspects of informal care in the home. Here, we argue, the role of mobile media (and its attendant datafication) can help to provide an ambient sense of autonomy from a distance - what we called "friendly surveillance" (see Chapter 1 and 3).

In contemporary Japan we are starting to see emerging forms of informal care and housing that no longer adhere to traditional familial ties. Instead, we are seeing new forms of Co-futuring Kinship. As argued in Chapter 1 and 2 , the digital plays a key role in creating continuity, rather than disruption, in practices of kinship. We illustrated that through the context of LINE (Chapter 2), familial kinship can be reinforced through digital relations. Moreover, the digital can also create forms of kinship between non familial members.

Take, for example, 37-year-old female graduate student Akiko who had divorced her husband and lived in a shared house with a cat "Mii" in the Tokyo metropolitan area. In the shared household there also lived an older administrative woman in her 6os and a graduate student at the same university. Her parents lived in the Tohoku district. To come back to her parents' home, it took over three hours from Tokyo by airplane or Shinkansen (bullet train). Her parents used to run a construction company for years but they semi-retired when Akiko's older brother inherited a business several years ago. Her father Masaru, 73-year-old, traveled to a local hospital but also a special hospital in Tokyo several times a year because of kidney disease. Her 68-year-old mother Yoshie was healthy and supported her husband for a long time.

However, Yoshie went to hospital for pneumonia. Masaru was a typical traditional husband who was committed to his work and left all housework and childcare matters to his wife. For Akiko, Yoshie was a "super woman" who managed housework, childcare and supported their family business. If something happened to Yoshie, Masaru wouldn't cope. Once Masaru called Akiko at five in the morning to come back home. He said, "Mother might die so come home." Yoshie survived but the incident highlighted how much Masaru depended on Yoshie. Akiko's brother, Kouichi, helped with parents' daily life when he wasn't busy managing the business. 
Given the circumstances, Akiko decided to come home more frequently to take care of her parents. Things had changed a lot from when Akiko used to live in Tokyo with her ex-husband. But since the divorce and her parents' decline in health, Akiko had to make a few big changes in her life to ensure that her parents are cared for. When Akiko wasn't there, she kept a friendly eye on Yoshie through LINE. Kouichi's children taught Yoshie to use her smartphone and LINE. Yoshie was always looking forward to seeing Akiko's life in Tokyo on Facebook and LINE. Akiko was mindful of what she chose to select and share with her mum.

For Yoshie, seeing Akiko with a job and research made her happy. Yoshie sent messages and stamps on LINE to Akiko as soon as she read Akiko's posts. Akiko felt that it had become a great deal easier to get in touch with her mother after she started to use LINE. Calling was sometimes bothersome. But on LINE it was easy to share mundane matters and fleeting feelings. For Akiko, the stamp feature of LINE was most useful as it enabled them to easily “confirm the existence of each other." Initially Akiko didn't like LINE that much, however, the cuteness of the stamp "softened" the communication between her and her mother — which allowed for informal care at a distance.

As discussed in Chapter 2, the rise of paralinguistics allows for new ways to articulate emotional and often tacit ways of caring at a distance. This new vernacular can allow for intergenerational modes of care to be fostered. Paralinguistics in the form of LINE stamps allows for playful experiences between mother and daughter. As Yoshie said in one post, "It's fun to send you stamps. This stamp reminds me of your childhood." Akiko said, "I am 37 years old now!!” Yoshie says “I know ... I am an annoying mother...”

37-year-old Serika, who worked in an advertising agency, had a different story about care at a distance. She lived in the Tokyo metropolitan area with her husband and was expecting their first baby in six months. Serika's parents were divorced but she had a good relationship both with her father and mother. Her father had been struggling with cancer for two years and was now in hospital at the terminal stage. Serika, his only daughter, traveled to hospital to take care of him on a regular basis.

As part of her job, Serika used smartphone apps. But, for a long time, she resisted using them in private life. She still used SMS to keep in contact with her husband. For Serika, she hoped for a watching camera app for babies and so she could give her 74-year-old father Kunio a way to be part of the baby's life during his last stages of cancer in which he was bed-ridden. Serika thought that these kinds of watching systems could help foster a sense of safety, especially with more older adults who lived alone. Serika said "seeing 


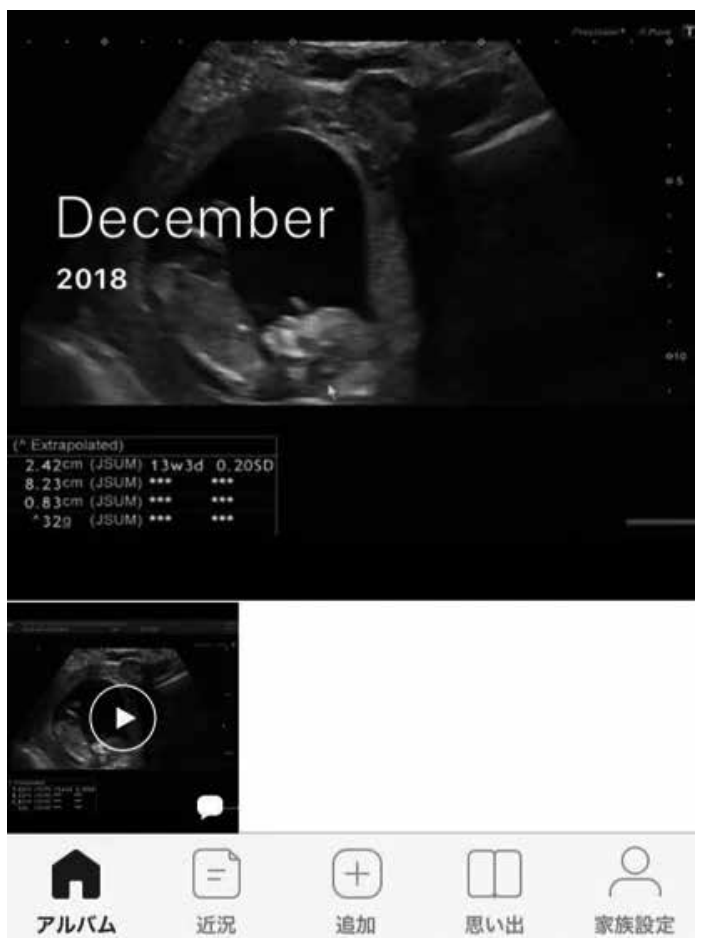

Figure 9.1: A moving echo image of the embryo provided by the gynecologist and shared with Serika's family members on Mitene

can create relief." For Serika, visual information had become increasingly essential for families who lived separately.

Recently Serika started to use a family album app "Mitene" (take a look) as recommended by the hospital. It enabled Serika to share an image of her embryo sent by the gynecologist among family members who registered in the app. The app recorded the baby's growth by picture or moving image, which enabled other family members to watch the baby's growth at a distance. This phenomenon is what Tama Leaver calls intimate surveillance (2017) - a practice that has, according to Leaver, ramifications for future generations in terms of digital legacy and data control.

\section{Digital Care}

Although Akiko still felt young, the idea of dying alone frightened her. Even though she lived in a shared house, she hardly saw her housemates. Akiko 


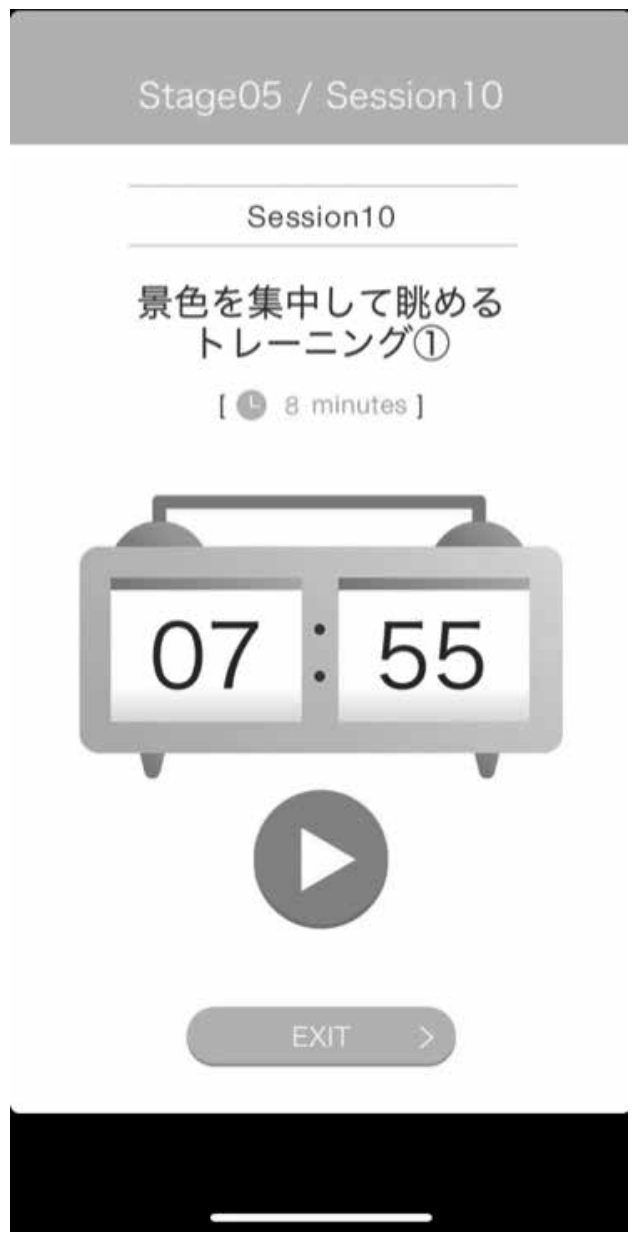

Figure 9.2: Screenshot of a meditation app, which gives instructions according to the user's heart rate. The text translates to "training to look outside with concentration"

became interested in buying an Apple Watch to help monitor her health and heart-rates. She also started using a meditation app to help with anxiety and stress issues. After facial registration occurred using the camera, the app automatically measured heart rate and gave instructions for something that must be done in relation to these biometrics. When thinking about mobile apps, Akiko hoped that the digital data about her body might provide new understandings into her health.

Like Akiko, Serika also liked the idea of an online doctor who diagnosed based on facial recognition via phone camera or advised according to biological data through an app. Serika had used a few apps for monitoring 
health - an app which forecasted ovulation according to body temperature (when she was taking infertility treatment) and also an app for weight control.

Since becoming pregnant, Serika used her smartphone constantly to look up facts about giving birthing and childcare. If she had morning sickness or some such condition, she researched it using her phone. She found it gave her more relief than a book or asking another person. Serika liked that she could search different responses and conditions in detail, commenting that "the smartphone is like a teacher about childcare for me." She noted that she relied on the phone because she didn't live with her mother. Getting online was faster. For Serika, as an expectant mother, she used the phone like a doctor-monitoring her child's health and notifying her of any concerns.

For Serika's 74-year-old mother Yurie, "digital health" had a different meaning. She was new to smartphones having only purchased recently. Living with her grandson, Yurie had learnt to use LINE from him. For Yurie, social media like Facebook and Instagram concerned her-she felt strangers would invade her privacy. Recently she had hurt her back and found herself using the new smartphone for enjoying drama on Netflix and contacting friends on LINE. And so, for Yurie "digital health" entailed maintaining mental health by using the smartphone for entertainment and communication with her close friends and family members.

Similarly, Yurie's 73-year-old friend Masami uses tablet PC for entertainment. After getting divorced in her 40s, she worked as a full-time until her late 6os. She retired at 70 when she started to feel she had less energy and strength. At that time, she also gave up driving. Since then, Masami often spent her time at home. On weekdays she went to an orthopedic doctor twice a week to care of her knee and to a culture center to learn Japanese calligraphy once a week. She also sometimes went out for lunch with her friends or her daughter. Otherwise, Masami watched TV at home. Her son recommended she play the game reversi on tablet PC to help prevent cognitive decline. This was her first tablet experience. Even though smartphones were attractive, Masami thought they looked difficult and so preferred to use her featured phone. In the beginning, she felt confused about how to use it - gradually she mastered it and enjoyed playing games. Getting a higher score brought her joy and she felt satisfaction. She also liked the idea that the game was helping her cognitive health.

Retired 64-year-old Hiroshi also used digital media to maintain his mental and physical health. He was very interested in new technologies such as mobile devices and the smartphone because of his previous job. Hiroshi had been an iPhone owner since its release in Japan. Hiroshi used various apps - such as LINE, weather forecast, and an app provided by his town which reminded him of garbage collection day. In addition to his iPhone 


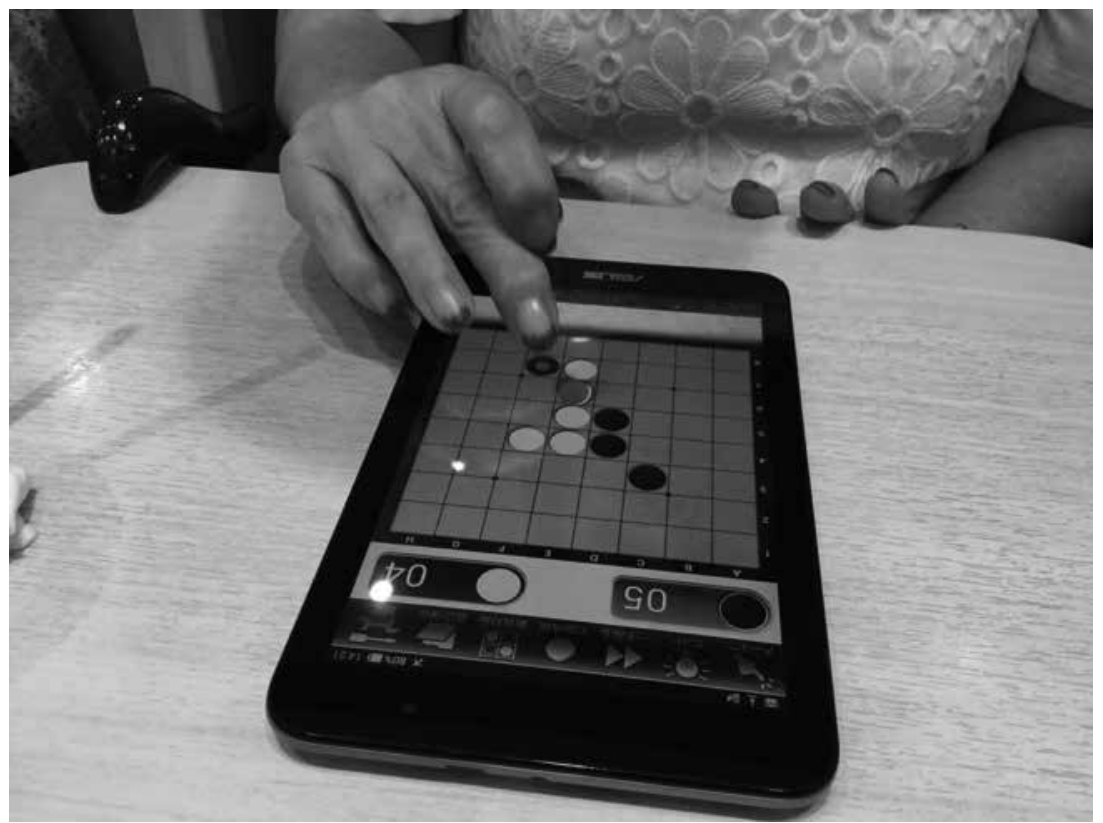

Figure 9.3: Masami is playing game, reversi, on tablet PC

Hiroshi had an Apple Watch to check his schedule and to monitor his daily exercise. He liked playing games but preferred to play on the bigger desktop computer screen. Hiroshi played simple games like Tetris and solitaire. Hiroshi admitted "I cannot play young people's games.”

While Hiroshi enjoyed the more retro games, he was mindful of their role in helping to maintain cognitive health. As someone that previously worked in media (at a cable television company), Hiroshi enjoyed the role of technology for relaxation and reflection. Here he differed from some of our female participants in his age group as he didn't use the technology very much for socializing.

Through our fieldwork in Tokyo we developed insights into some of the complex and ambivalent ways in which intergenerational care is playing out in different data trails. While younger participants talk more about present practices and perceptions about the future almost seemingly, our older participants seemed much more circumspect in their observations. Moreover, there still seemed to be some implicit notions of agism with the intergenerational discussions around technology. However, the use of mobile apps for care at a distance cannot be underestimated.

There was also less concern around the role of data and digital legacy than in the Shanghai and Melbourne contexts. In Japan, this was partly to 


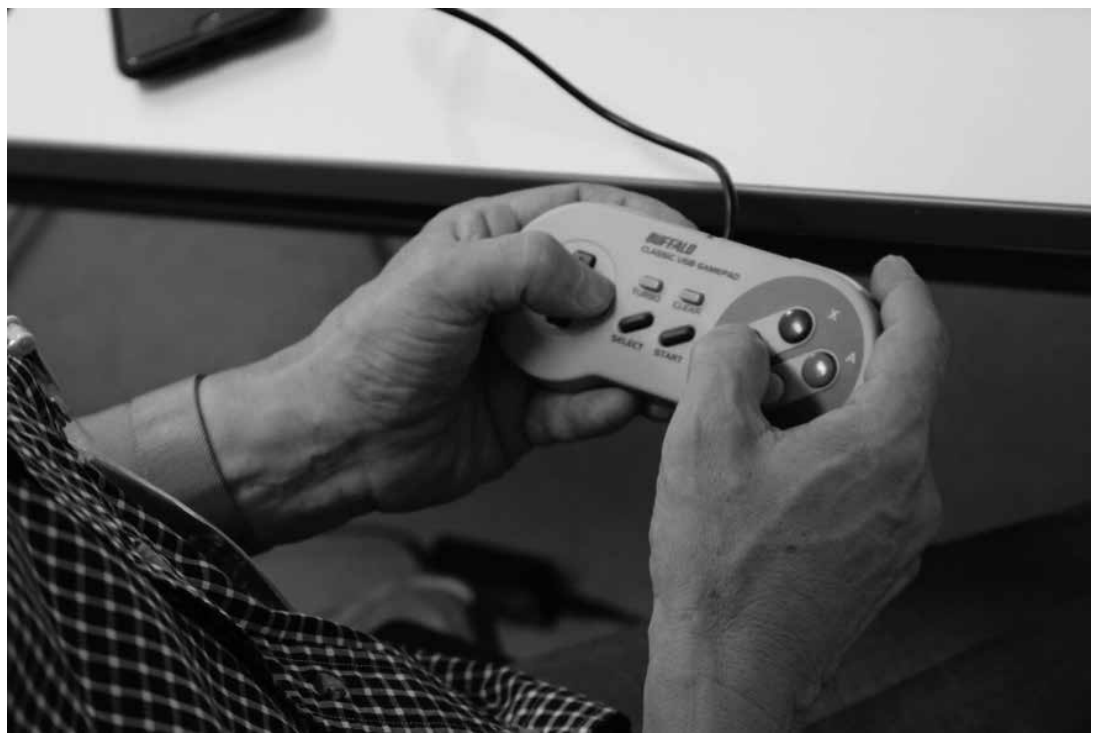

Figure 9.4: Hiroshi likes playing "old" games on desktop computer

do with underlying Buddhist spiritualism whereby this life is conceived as one in many. It was also due to the lack of palpable impact of data trust scandals (i.e. NHK Fukushima disaster cover up) in Japan in which social media like LINE, rather than Facebook, dominate.

In our Japanese fieldwork, the growing role of technology to assist the burgeoning super-aging populations was undeniable. As aforementioned, much of the emergent technology work was grappling with phenomenon such as aging populations in remote areas. The use of AI robots in aged care facilities to explore alternative care scenarios was a growing field of research and practice (Yamazaki et al. 2018). AI Robots like Hiroshi Ishiguro's "Telenoid" were being pivoted in dementia care settings to see if robots could help with memory and sociality. While STS scholars such Judy Wajcman rightly argue that robots and AI will never be able to master the slow and mundane rituals of care that humans do so well, how they operate in intermediary ways - even if to just give reprieve to care workers - will continue to be questioned into our Co-futuring Kinship.

\section{WeChat and Informal Care}

In Shanghai we find parallel practices emerging. While not experiencing super-aging yet, the country's thirty year one-child policy is starting 
to have ramifications in caring for older adults. With only one child to care and work, stress on informal care systems will begin to grow. Moreover, with the rise of technological surveillance being used by the government to implement automated issues such as social credit, the role of smartphones for self-tracking such as monitoring health takes on different dimensions.

According to the statistics of China's National Bureau of Statistics, there are about 240 million elderly people in China over 60 years old, and about 160 million people over 65 years old (Jiao 2018). With the aging of China's population, the elderly population has become the largest demand for health care services, and its health needs and medical security have become the hotspots of all circles (Chen and Xu 2011). At the end of 2018, China's internet penetration rate reached $59.6 \%$, and the number of users reached 829 million. However, aged users over 60 only accounted $6.6 \%$ of the internet users (CINIC 2019).

The introduction of mobile applications for health or medical dimensions was introduced late to China in 2011. However, since 2011 digital health applications have developed to include "Safe Doctor," "Meiyou" and "Xin Yang" (Chen 2018). WeChat dominates the mobile application scene with more than $80 \%$ of smartphone users in China deploying it. WeChat not only has social functions, but also features mobile health — such as pushing health information, making appointments, etc. The mobile health function of WeChat is predominantly used by middle-aged and older adults to follow health-related public accounts, discuss health problems in WeChat groups, as well as forward relevant health pushes, among others, which facilitates the information flow and exchanges among middle-aged and elderly people in China (Xu et al. 2018).

Simultaneously, the Chinese family structure has undergone a large change in recent decades with the majority of families transformed from an extended family structure (i.e., grown children living with older parents) to a nuclear structure where grown children live on their own (Gao et al. 2015). In this phenomenon, the older adults and their grown-up children often live a long distance apart and can only communicate with each other through mediated channels. In major metropolitan cities like Beijing and Shanghai, older adults are more likely to be economically independent and the co-residence of older people with adult children is declining (Zimmer et al. 2007; Logan and Bian 1999). According to a national survey on the living status of older adults, the average rate of the "empty nest" (KongChao) family has reached $49.7 \%$ in China's urban areas, with a $7 \%$ increase since 2000 (China National Committee on Ageing 2007). Given these factors, 
mobile-based social media platforms like WeChat have become a crucial channel for intergenerational caring and emotional support among longdistance family members across China.

China's current mobile-health platform can be classified into five types according to their functional characteristics-medical product e-commerce platform, professional information inquiry platform, medical consultation platform, sub-function function platform, health management and health care platform (Prospective Industry Research Institute; Wang and Wu 2014). In terms of technology development, studies in China have established a chronic disease management software model for hypertension patients to analyze user data (Qi 2017). Li Yangzi developed a telemedicine monitoring mobile medical platform based on the Android platform. Patients can conduct an inspection report inquiry, online diagnosis and treatment, and obtain health guidance on the platform $(\mathrm{Li}$ 2014). Xing Jin (2017) introduced the mobile medical system and mobile doctor assistant of Xuanwu Hospital of Capital Medical University (Jin and Fei 2007).

In user behavior research, some scholars have explored the influential factors of mobile health service acceptance behavior of middle-aged and older people in China. The results show that the vast majority of middleaged and older people have some anxiety about new technologies, which negatively affects their adoption intention to mobile health. Perceived ease of use in the process of implementing mobile health services has proven to be an important factor in influencing the adoption of mobile medical technology, which in turn, emphasizes the improvement of convenience and usability are needed to eliminate the anxiety and worries of middleaged and old users on new services (Mo and Deng 2015). For example, Liu Yongjun's research explores factors of mobile medical payment diffusion. In a discourse analysis of the website Hao Daifu (a popular online medical platform), Zhang Xiaofei established an online medical service adoption model and found that medical habits, silent costs, conversion costs and privacy protection create a certain hindrance to users' practice of online medical services (2014).

Sun et al. (2016) also focus on the development trends in Mobile Health and its clinical applications for the prevention and treatment of diseases, especially on the operation model of m-Health. They suggest the unified practice doctors' model, in which all of the doctors do not need to provide face-to-face services for patients (Sun et al. 2016). In China, Mobile health service app such as Chunyu Doctor has been studied to show its ability to enable general users to look up symptoms or to consult with professional 
doctors free of charge, as well as allowing professionals to communicate with health consumers.

Many new models of human-computer interaction (HCI) have been launched in various parts of China to respond to aging populations. Wuzhen has established an $\mathrm{O}_{2} \mathrm{O}$ platform for the aged care community to provide home-based early warning, emergency assistance, and health management services for the elderly and families. The online platform is mainly for the elderly to install smart home care equipment, remote health care equipment, SOS calls and alarms, and to use Alibaba Cloud server and WeChat for remote management.

The first "virtual nursing home" in Xi'an was launched in Lianhu District in March 2018. This is defined as the "nursing home without walls." Through the call center, it provides socialized old-age service subscription, planned appointment, service scheduling and Service supervision for the elderly groups in need (Zhao 2018). The need to design for complex sensory dimensions for older adults is vital-especially in terms of declining mobility, eye sight, and hearing impairments (Xu 2016). The use of robots for social interaction and mundane activities is starting to emerge — but only nascently when compared to Japan.

In fieldwork with our older adults, there was little understanding into how locative media within the apps functioned. However, many were concerned with perceived notions of privacy and trust. As noted in earlier chapters, the notion of privacy was culturally-specific - Japan had its own understandings of privacy that differed from the Chinese or Australian participants. Also, the significance of intergenerational understandings was also heightened as they were unidirectional. Often older adults were informally taught by their adult children, in particular ambient ways of being with the media that they were not necessarily attuned to.

Let us begin with our older adult participants. In Shanghai, popular digital health applications usage and attitudes among the older adults include WeChat sports and Xiaomi bracelet (self-track monitor). 51-year-old Li Wei was a government official in Suzhou. While Li Wei used a Xiaomi bracelet for a short period for tracking his personal health, he found that it wasn't very accurate and so he changed to WeChat sports. Li Wei said confidently that he didn't feel the need to use other apps because he was still very healthy.

Li Wei checked into the WeChat sports app at least once a day. This app kept track of his exercise and gamified it through ranking among friends. Li Wei's daily exercise was subject to the weather-if the weather was cold, he walked 6 ooo steps a day; when the weather was warm, he could walk 
12,00o steps each day. He admitted that the app changed his lifestyle habits and how he viewed health in an everyday context. Before using WeChat sports, he decisions to exercise were based only on his personal habits, like walking for one hour each day. After using WeChat sports, he made use of the app to monitor his exercise record and this became his "new habit." If he didn't walk someday, or if the walk number didn't meet his standard step count (e.g., 1200o), he felt that something was amiss.

However Li Wei has his doubts about other digital apps-especially self-tracking apps. He also commented that people should be mindful of "being kidnapped" by technology. When asked to explain further, Li Wei said, "when the digital is involved in our lives, other non-digital aspects of the body can be ignored. Some people will become reliant on digital indicators-like being kidnapped. If the indicators are not normal, we might feel more pressure, so we need to balance well."

For 54-year-old mother Biyu, hospital apps were useful to download when needing to see a doctor. As Biyu noted "for example, if I want to go to Zhongshan Hospital to see a doctor, I will download the Zhongshan Hospital app to get a registration number and make an appointment, which is quite convenient." In addition to hospital apps, the WeChat public account platform has become another popular channel for the Chinese older adults to make an apportionment to see a doctor. Biyu preferred using hospital apps as she was afraid to disclose privacy on WeChat. She also tried some self-tracking and testing apps—-like blood pressure meter apps—but she had concerns about the accuracy of the digital health applications. For instance, she did not trust the accuracy of the blood pressure app. "Compared to these apps, I trust the tests and measurements from traditional hospitals more," explained Biyu.

In fieldwork, there was a great discrepancy between young and older people attitudes towards digital health mobile apps. 69-year-old Aunt Chen said that she preferred to go to the hospital for regular registration and checkups, while her daughter favoured registration and checking on the WeChat platform. Biyu believed that while digital health was promising for young people, it was less realistic for older people. "In fact, many of us still like traditional ways - for example, I have learned how to pay by WeChat, but I am still paying with my wallet... Many of my friends in this generation feel that it useful to have digital options but we would hate for it to be the only option."

Increasingly WeChat plays a central role in many aspects of Chinese everyday life. Health information is no exception. There are many ways people can obtain information-WeChat group, public accounts, and moments. 
For Li Wei, "Now I am getting older, so I pay a lot of attention to health information. I can't completely control my mouth, but I try my best to keep a light diet and eat more whole grains. So, I need to know more knowledge about how to make a healthy eating and lifestyle." He subscribed to some health-based WeChat public accounts_-including "Wen Xiaoshu says" and "a guide to make a healthy life" — which sent him a notification every day.

Li Wei also joined some health knowledge groups via the introduction of friends — such as "Dadao Medical Center" and "Suzhou Health Sharing Group" - from which he also received updates about how to maintain a healthy life. Li Wei said that some professors and doctors were also active in these groups, helping to solve people's questions and inquiries, which he felt was quite useful and efficient. He also often forwarded and shared this information and knowledge to his own family group. Although he found these messages quite rich, he also doubted their credibility. He noted, "these messages are not entirely accurate, and even some are rumors." Li Wei thought it to be very important to check the health information but also admitted the difficulty for older people. Regarding the influence of WeChat channels, Li Wei said:

For much older groups like those are over 70 or 80 , the new media has no influence on them. However, for our age (50-60), the influence nowadays is still relatively large. Digital media like WeChat public accounts and groups are the main channel for me and my colleagues to know about the health information. And secondly, we can also exchange our experience about health issues... For example, cancer and diabetes patients can build a group specifically to organize some activities to discuss the current treatment methods, similar to the offline clubs and associations, which can helpful.

WeChat also plays an important role in informal ways of caring at a distance. This care can take the form of everyday sociality in helping with healthcare issues. Li Wei had 6oo-700 WeChat friends and 40-50 WeChat groupsincluding classmate groups, family groups, activity groups and co-worker groups. Talking about WeChat groups, Li Wei believed that the family group promoted long-distance communication between family members and facilitated the guidance and care with his daughter. Li Wei usually sent articles to his daughter, mainly about social value orientation and health information, in hopes that his daughter would pay more attention to health. He also utilized WeChat video once every few days with his daughter who was studying in Hong Kong. 


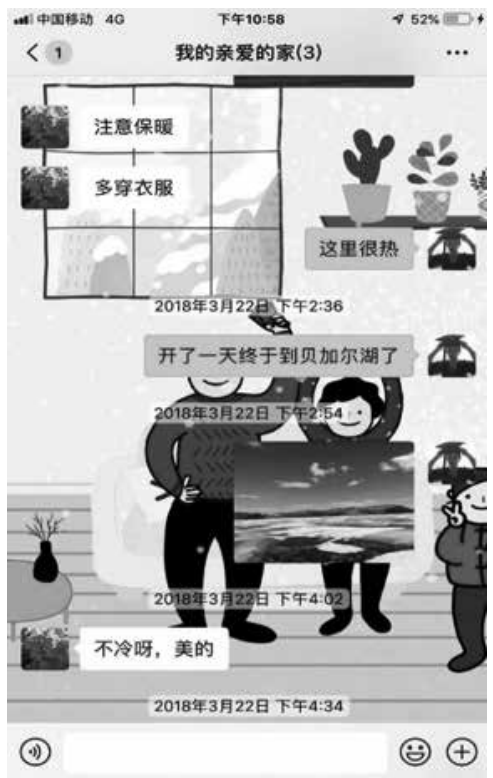

Figure 9.5: Screenshot of Li Wei's family group chat

Li Wei said that his wife was more actively involved his daughter's life — she asked her daughter more questions about her everyday life, taught her to cook through WeChat, reminded her about weather changes when she would need to travel home early. Once, through the remote guidance on WeChat, the daughter actually surprised them by making a table dish of garlic, cucumber, fried beans, braised prawns, steamed fish, etc. Li Wei was very happy and praised his daughter on WeChat. "Take this opportunity to practice cooking, and I look forward to your cooking skills when you go home."

Care and watching is often unidirectional. Adult children also use social media to watch their parents' lives and keep a friendly eye on them. As the only child, their 24-year-old daughter would also use WeChat to keep abreast of her parent's lives and health. She was worried that her parents were lonely when she was studying abroad. Therefore, she often sent updates to her family through WeChat, including photos and interesting stories. "I will let them know what I am doing every day, and it is another companion to them," explained Li Wei's daughter. This story of the use of WeChat's for care at a distance is common in contemporary China.

Different from Li Wei 's family, Biyu rarely sent health information to her son or to her family group. "I don't want to bore my son. I prefer to send positive information." Biyu explained that she seldom sent health 
information to her son because it was difficult to judge the credibility of much of the health information online. She also suspected that she had been blocked by her son because she couldn't see her son's WeChat moments. "I think he must have grouped me into a special group and blocked me from some content. But I didn't ask him about it." She explained her special communication model with her son, "Only when I have something urgent to communicate, will I make contact."

Aunt Chen said that WeChat had become one of the most important bonds to maintain family ties: "This kind of caring is very important, and it keeps the family together... For example, if I do not send any WeChat news to my daughter, my daughter will worry and will immediately give me a video call to confirm and vice versa." If there was any problem in daily life, Aunt Chen would also inform her daughter through WeChat, especially relating to situations about health status.

For example, recently, Aunt Chen got up and found that the towel she used had blood on it. After thinking for a while, she immediately took a photo and sent it to her daughter through WeChat. They talked through what it could be, problem-solving together. This was a typical long-distance health communication between two generations via WeChat. "My daughter was afraid to see the pictures and worried about whether there was something getting worse in my body. She kept checking my situation and gave me suggestions." This concern from her daughter made Aunt Chen feel better. She believed that WeChat is not only an essential tool for her family to express caring but also, for informal modes of healthcare.

As we saw in our Shanghai fieldwork, generational differences in attitudes towards data and health prevailed. Older adults, while using WeChat health discussion groups and hospital apps to book appointments, were clear that they didn't want the technology overtaking face-to-face care. Here, their thoughts echoed the findings in Pols' telecare study about the role of technology to enhance, not replace, face-to-face care (2012). Our younger participants were much more active in their use of tracking apps for self-care and were keen users of WeChat for familial care at a distance.

\section{Careful Apps in Melbourne}

Unlike Tokyo and Shanghai which saw main social media LINE and WeChat being used to not only care at a distance but also to choreograph ways of thinking about data and health, in Melbourne we find many of 
our participants using a diversity of media and techniques. Returning back to Jasmine, we find a participant whose practices have been greatly affected by the Cambridge Analytica debacle. After being hacked she is now vigilant about privacy settings for herself and her mum. As Jasmine noted:

My mobile media practices — that's my laptop, my tablet, and my mobile phone-have changed quite a bit... My phone, I don't use my phone as much; I don't check it as regularly. I still check it throughout the day, but I definitely used to scroll through Facebook a lot more. I took the app off my phone-after Cambridge Analytica and all of that stuff that happened last year. My mum's account was also hacked, and somebody started contacting her friends-I used that as an excuse to go through her phone and her Facebook profile and my Facebook profile to de-friend a lot of people and to just tighten down my security settings... At first, that was quite mentally difficult (without Facebook app), because I had to demarcate, OK, yes, you can do this; no, you have to stop, no you have to turn this off, no you can't use that. So that was a mentally exhausting to try to manage, but after a little while it became good habits.

When discussing her changes in her media practices, Jasmine reflected upon the way in which generation shapes media use. For Jasmine, she viewed mobile media as an important part of older generations having social connection-especially in the case of her mum. As Jasmine said:

I'm mid-3os and grew up in a time where we didn't have internet, and then we did have internet, and then we had mobile internet. So, I think for our generation, we've got a mix of addiction, discerning use, built into the routines in a way that we don't really think about it. So, I just hope that there will still be positive routines around social and mobile media use. My parents' use is different. For my mum, I think social media and her tablet has been great for her. She's still working, so when she comes home from work, she's still got her friends overseas, her relatives overseas that she chats with or she sees their posts. I think devices are good for aging populations in terms of diminishing social circles and less activities to do. I think they're a good source of company.

For Jasmine, the generational differences between her mum and her own practices were very much in terms of social connection. This importance 
of social connection had been a key aspect in aging well. For Jasmine, WhatsApp was crucial in keeping connected with her family in Australia and overseas. She observed:

We also have a family WhatsApp group, and that's a touching base, that's well wishes; it was just Chinese New Year, so we sent some images for that. So, yeah, I think they're gentle, just touching base with images and organization to catch up, and things like that. So that will be our informal care with social mobile media use. For care at a distance, yeah, for mainly that friend who lives in London, and it's the banter, humor, gossip, images; I think we also use Instagram quite a bit together. We direct message each other with other people's posts that we find amusing, usually cat posts, or if there's make up, or fitness, or if we're planning a trip, an image from that destination we want to go to.

For Jasmine, self-tracking apps were not of much interest. She noticed that her younger family members - especially female - were active self-trackers and used many apps. She expressed concerned that some of this micro-managing of the body could lead to unhealthy body obsessions - reflecting "beauty apps" discussions by Ana Sofia Elias and Rosalind Gill (2017). As Elias and Gill argue, such apps "offer a technology of gender which brings together digital self-monitoring and postfeminist modalities of subjecthood" in ways that are neoliberal $(2017,59)$. In building on the work in feminist surveillant studies they argued that such gendered self-tracking apps create "intensification, extensification and psychologization of surveillance" (Ibid.).

Indeed, much of our discussions with Jasmine - as with other Melbourne participants - foreground more ambivalence towards apps than in Tokyo and Shanghai. As Jasmine noted, whilst she and her friends had made a concerted effort to "pull back" from apps and datafication, Jasmine recognized that the future would be even more "datafied." As she noted:

I think there'll be a lot more interventions. I think there'll be a lot more hard-selling of apps, either to collect data, or to push a certain kind of healthy lifestyle. And those, I think, will become normalized as well. I'll probably download some stuff and then either embrace them into my routines or reject them [...] So, I think there will be a lot more interventions into pushing people to maintain healthy lifestyles and to collect data on them, and then these sorts of digital interventions will be normalized so much they become invisible, like social media was a few years ago. But now, a bunch of us have pulled back from that social media use as well. 
Like Jasmin, father Damian also noticed a continued growth when he used WhatsApp for discussions with family and friends to keep constantly connected. He saw the role of data in everyday life as a "double edged sword" and thought that the technology made for more frequent but shallower connections. As Damian noted:

Yeah, I think it's a double-edged sword and I don't know which way it'll go. It simultaneously connects and disconnects people. So, on the one hand, people have I've started to realize is that they are so connected with so many people by seeing them on Facebook. And yet it feels like people are catching up face to face less. We are all so busy.... Technology makes us feel like we need to busier and then allows us to fill in the absences with Likes. So, I've just got ridiculously wide circles of relatively shallow friendships with lots of people.

For Damian, the future of data in his and his family life involved the need for media literacy. As he observed:

I think that there might be some sort of space to educate people and give them really concrete strategies for how they engage with their technology to make sure that they're using their technology, not vice versa.

For 50-year-old Maria, wearable technologies like the Apple Watch had "released" her from her phone. She commented on how she was able to keep track of her fitness - which was important to aging well-without much effort. Maria was so happy with the Apple Watch that she had bought her 70-year-old mother one too-and then spent a week teaching her interstate mother how to use. Maria found comfort in features like heart rate monitoring apps-knowing that if a problem arose for her or her mother others could be alerted. She wondered if such conditions as dying from a heart attack could be avoided with such monitoring devices. Six months on from the purchase, Maria still received calls from her mother about how to work something or how to fix the Apple Watch. Maria joined her mother to the fitness app Strava so they could compare walks co-presently. As Maria noted:

My mum and I loved walking together. But being interstate means we can't do that very often. But having us both on Strava means we can give each other kudos and comment on each other's walks to give encouragement as an excuse for a conversation. I will ask her about what the walk was like.... 
Something that the Strava trail doesn't really show. It also makes me feel good that I know my mum is still fit and active. I think she will live to 100.

For 6o-year-old Amy, apps like WhatsApp were important in maintaining social connection with friends. It's often used to coordinate a social event with key friends, or to talk about key interests like films. While Amy's adult children used a lot of media and have taught her to use technology like iPads, she saw the dominance of mobile media slowing down. As Amy said:

There was a time when the kids were always with their phones, constantly on screens, texting each other while in the same room. I guess young people always find ways to challenge older generation's ideas and beliefs. But I have noticed a pull back from those behaviours-especially given all the stories about fake news and Facebook hacking. It's good to see some reflection. Well, at least it appears that way! My daughter recently showed me how my smartphone tracks my walking (default Health app) which was an eye opener. I'm still unsure how much of my life is being "captured" by this device... and what it will mean after I pass away.

50-year-old Mark was deeply concerned about data trails and digital legacy. In particular, he was worried about his parents who had started to use apps like Facebook on their phone to keep in contact with his sister overseas. Mark worried that they weren't aware of the darker side of datafication. He frequently asked them not to post pictures and to be careful what things they recorded on Facebook.

For Mark and his friends, being mindful of how their data could be used against them while they were alive, and also dead, was of major concern. When completing his first will Mark ensured he had a digital legacy component to it so as to ensure that his data could be accessed by loved ones, and not owned and reappropriated by corporations. This area of digital legacy is a growing area of interest and concern (Cumiskey and Hjorth 2018; Kaskett 2019).

In the Melbourne fieldwork, the use of apps for informal or formal health was less prevalent. However, the use of apps for informal care (at a distance) remained salient across the three sites. Here we can see the different ways in which health and care are manifested in everyday life subject to notions of trust. In sum, they are about quotidian notions of care and health shaped by generational, cultural and linguistic contexts. In particular, in Melbourne we noticed the biggest impact of the Cambridge Analytica debacle with many like Jasmine deleting their Facebook app on their phone and being 
more mindful of the darker side of quotidian datafication. In all locations, generational perceptions differed in terms of trust and understanding. But more research is needed to be done in this dynamic area.

\section{Conclusion}

In this section of the book we have explored some of the emergent ways our participants reflected upon social mobile media in everyday life through the ubiquitous rise of datafication through apps. In this assemblage we find various forms of ambient locative media. As we have discovered, while locative media was once about tracking through public spaces and places, through the rise of self-tracking we see the notion of locative media being recalibrated in ways that cater to more internal types of mapping. As we start to see, the rise of datafication can allow for us to explore the emotional and social cartographies of being in the world. This can be understood as feeling locative media - that is, the tacit and sensorial ways in which techniques of locative media-where location involves both internal and external cartographies-are being deployed in everyday life.

As this chapter has explored, feelings and perceptions about data and care differ across generations, cultural and linguistic contexts in ways that suggest more work is needed into the dynamics of Co-futuring Kinship. As we have argued through the kinship rubric, these practices are about a process of continuity rather than disruption to everyday rhythms. Having explored the beliefs and opinions of older adults in Chapter 8 we now can better understand how differences between and through the generations' use of media occur. We reflected upon how younger participants are co-futuring around applification and its relationship to family and kinship.

In this chapter we also started to hear participants' concerns around their data being used in unintended ways-in past (i.e. Cambridge Analytica debacle, NHK Fukushima disaster), present and in the future (data of the dead). In particular, participants voiced issues around what happens to their data and loved ones when they die. Notions such as digital legacy—having a provision in legal wills around ownership of data post-death — are becoming more and more apparent.

In fieldwork with participants over the three-year period, the rise of self-tracking and conspicuous journaling for parents at a distance played out. As discussed earlier, their digital media has increasingly played a pivotal role in navigating expressions of emotion, particularly intergenerationally when physical distance is an issue. As Lupton (2019) has noted, the rise of 
self-tracking has demonstrated how digital narratives can have material ramifications for users. She highlights that such QS practices make users more aware of their movement and its impact in the world.

For Lupton, feminist material approaches can provide insight into the ambivalences around self-tracking which include pleasure and burdensome elements. It has allowed for more "affective" and "sensory" understandings of movement, data and the everyday. Lupton points to ethnographic fieldwork around self-tracking in which digital devices can "monitor embodiment and selfhood, the mobilities and emplaced dimensions of these practices and the role of data visuality" (Lupton 2019, 68; Pink and Fors 2017).

In our fieldwork we have focused on the relationship between tracking and self-tracking intergenerationally as care at a distance. In this fieldwork we found how families and intimates could use data ambiently to keep a "friendly" eye on each other. Sometimes, these tacit forms of ritual and care moved ambiently from the background to the foreground in everyday life. And as we move towards datafied futures (Co-futuring Kinship), quotidian care at a distance can help us understand some of the ways in which data is playing out in intergenerational digital literacies around care.

\section{References}

Anonymous. 2011. Chunyu Doctors. http://www.chunyuyisheng.com/. Accessed 2 March 2018.

Akiyama, Hiroko. 2010. "Choju jidai no kagaku to shakai no kousou." Kagaku 80 (1): 59-64.

Cabinet Office, Government of Japan. 2018. White paper on aging society 2018. http://www8.cao.go.jp/kourei/whitepaper/w-2018/zenbun/3opdf_index.html. Accessed 2 March 2018.

Chen, Youhua and Xu Wei. 2011. "The health status, welfare needs and prospects of the elderly population in China [J]." Population journal 2 (In Chinese).

China Internet Network Information Center. 2018. The 43th Statistical Report on Internet Development in China [EB/OL] (In Chinese), Report. http://www.cnnic. net.cn/hlwfzyj/hlwxzbg/. Accessed 2 March 2018.

China National Committee on Ageing. 2007. A follow-up survey on China's elder population in urban and rural areas, Report. http://www.cnca.org.cn/ default/content/contentllxw.jsp?columnid=iroot $1000010001 \&$ articleid $=$ 4028e47d16ec2fd9011719a71bdg044e. Accessed 2 March 2018.

Chen, Yujia. 2018. Analysis of the factors affecting the adoption of mobile medical care by the public. Report. Dalian University of Technology (In Chinese). 
Elias, Ana Sofia, and Rosalind Gill. 2017. "Beauty surveillance: the digital selfmonitoring cultures of neoliberalism." European Journal of Cultural Studies 21 (1): 59-77. DOI: $10.1177 / 1367549417705604$.

Gao, Qin, Ebert, Daniel, Chen, Xing, and Yao Ding. 2015. "Design of a mobile social community platform for older Chinese people in urban areas." Human Factors and Ergonomics in Manufacturing \& Service Industries 25 (1): 66-89. DOI: 10.1002/ hfm.20523.

Han, Xinnian, Shi Wei, Li Hui, Wang Wei, and Meng Fanyuan. 2017. "Research on key technologies for the construction of regional medical information service platform [J]." The world's latest medical information digest 17 (71): 208-206 (In Chinese).

Iijima, Katsuya. 2016. "Early prevention strategies of 'frailty' based on local support network." https://www.mhlw.go.jp/file/05-Shingikai-1080100o-Iseikyoku-Soumuka/ ooo0135472.pdf. Accessed 2 March 2018.

Inagaki, Kana. 2019. "Japan's demographics make good case for self-driving cars." Financial Times. 17 July. https://www.ft.com/content/382aef5c-a7d7-11e9-984cfac8325aaao4. Accessed 2 March 2018.

Iwasaki, Fusako. 2017. "Elderly Person and Frailty." Quarterly journal of welfare society $36(2): 1-16$.

Jiao, Yahui. 2018. "Four questions test elderly medical care [J]." China Health July (In Chinese).

Kanawaku, Yoshimasa. 2018. "Koritsu-shi (Solitary Death) and its Actual Situation." Journal of Nippon Medical School 14 (3): 100-112. DOI:10.1272/manms.14.100.

Kiwaki, Nachiko and Arai, Yasutomo. 2015. "Social Isolation of Elderly People in Japan: From the Viewpoint of Family Paradigm Changes.” The bulletin of the Faculty of Human Life Sciences 52: 65-74.

Li, Yangzi. 2014. Research and Design of Mobile Medical System Based on Android Platform [D]. Report. Wuhan University of Science and Technology (In Chinese).

Liu, Weiying. 2014. System design of walking leg series elastic actuator. Report. Harbin Institute of Technology (In Chinese).

Liu, Xizhou. 2014. Design and Implementation of Mobile Medical Management System [D]. Report. Central China Normal University (In Chinese).

Liu, Yongjun. 2015. Study on the Influencing Factors of Mobile Medical Payment Based on Innovation Diffusion Theory [D]. Report. Southern Medical University (In Chinese).

Luo, Qihua. 2015. User-centered design of wearable sports health monitoring products for the elderly [D]. Report. Guangdong University of Technology (In Chinese).

Logan, John R., and Bian, Fuqin. 1999. "Family values and co-residence with married children in urban China." Social Forces 77 (4):1253-1282. DOI:10.2307/3005876. Lupton, Deborah (ed.). 2019. Self-Tracking, Health and Medicine. London: Routledge. 
Ministry of Health, Labour and Welfare of Japan. 2018. Abridged life table 2017. Report, https://www.mhlw.go.jp/toukei/saikin/hw/life/life17/dl/life17-14.pdf. Accessed 2 March 2018.

Mo, Xiuting, and Deng Zhaohua. 2015. "Empirical Study on the Acceptance Behavior of Mobile Health Services for Middle-aged and Old-aged Users in Wuhan." China Health Statistics 32 (2): (In Chinese).

Prospective Industry Research Institute. 2016-2021. China Mobile Medical Industry Typical Business Model and Construction Design Strategy Analysis Report (In Chinese).

Qi, Yumeng. 2017. Research and practice of a mobile medical software design method for patient compliance enhancement [D]. Report. Zhejiang University (In Chinese). Xing, Jin, and Fei Xiaowei. 2007. "The Architecture and Application of Mobile Medical System in Our Hospital [J]." Chinese medical equipment 32 (10): 122-25 (In Chinese).

Xu, Huan, Sun Sihang, Lin Wosa, and Yang Yang. 2018. "Feasibility analysis of mobile health based on smartphones to urban elderly." Journal of Preventive Medicine Information 34 (6): June (In Chinese).

$\mathrm{Xu}$, Junheng. 2016. Immersive interface design of the grip measurement software for the elderly [D]. Report. Zhejiang University (In Chinese).

Wang, Peng, and Wu Wei. 2014. "Discussion on the current status and future development trend of mobile Internet medical applications at home and abroad [J]." China Digital Medicine (In Chinese).

World Health Organization. 2016. Healthy life expectancy (HALE) at birth. Report. http://apps.who.int/gho/data/node.wrapper.imr?x-id=66. Accessed 2 March 2018.

$\mathrm{Wu}$, Rusong. 2012. Design of interactive TV interactive interface for urban elderly [D]. Report. Jiangnan University (In Chinese).

Yamazaki, Ryuji, Shuichi Nishio, Hiroshi Ishiguro and Hiroko Kase. 2018. "Use of Robotic Media as Persuasive Technology and Its Ethical Implications in Care Settings." Journal of Philosophy and Ethics in Health Care and Medicine 12 (December): $45^{-58 .}$

Zhao, Chuanfang. 2018. "Discussion on the development of Internet + aged care service [J]." China Development, August (In Chinese).

Zhang, Xiaofei. 2014. Research on the obstacles to the adoption of online medical services based on the current deviation theory [D]. Report. Harbin Institute of Technology (In Chinese).

Zimmer, Zachery, Kwong, Julia, Fung, Xianghua, Kareda, Toshiko, and Tang, Zhe. 2007. "Child Coresidence among Older Adults Living in Beijing, China: Trends, Determinants and Transitions." In New Perspectives on China and Aging, edited by Ian G. Cook and Jason L. Powell, $5^{-28}$. New York: Nova Science. 


\title{
10. Conclusion
}

\begin{abstract}
In Chapter 10 we reflect upon a future rubric for imagining social mobile media and data for intergenerational care at a distance.
\end{abstract}

Keywords: futuring; aging societies; data for care

In this book, we have investigated the evolving intergenerational media practices over three years to reflect on the quotidian (and often invisible) forms of care at a distance enacted as part of contemporary Digital Kinship. As we have explored, within different cultural contexts we are seeing diverging forms of intergenerational perceptions and practices around media and care. Over the three years, we witnessed the growth of self-tracking health apps which are being taken up in diverse intergenerational ways. As we note, understanding intergenerational care at a distance is about complicating care beyond medical notions of health and social services. In sum, taking care seriously as part of intergenerational media practices.

In the introduction of the book we met Rika. And so, it seems fitting at the end of the book to revisit her, to see how her changing kinship relations reflected in her media practices over the three years. Rika, who had worked as a flight attendant for more than 11 years, quit her job in 2015, at the age of 30 . By the end she had accumulated the equivalent number of miles to fly around the world almost 100 times.

Being a flight attendant had been Rika's lifelong dream since she flew to the US at the age of 6 to see her father after her parents divorced. During the flight, all the flight attendants were kind, and this inspired her to become a flight attendant. However, after flying for many years, she gradually began to think about marriage and having a baby. When we carried out the first interview in Tokyo in 2014, she was 32 and was still looking for a partner with whom she could share a family. Three years later, Rika found a partner and had a baby. She recently quit her job to devote her time to her new child.

Hjorth, L., K. Ohashi, J. Sinanan, H. Horst, S. Pink, F. Kato, B. Zhou, Digital Media Practices in Households: Kinship through Data. Amsterdam: Amsterdam University Press, 2020 DOI 10.5117/9789462989504_CH10 

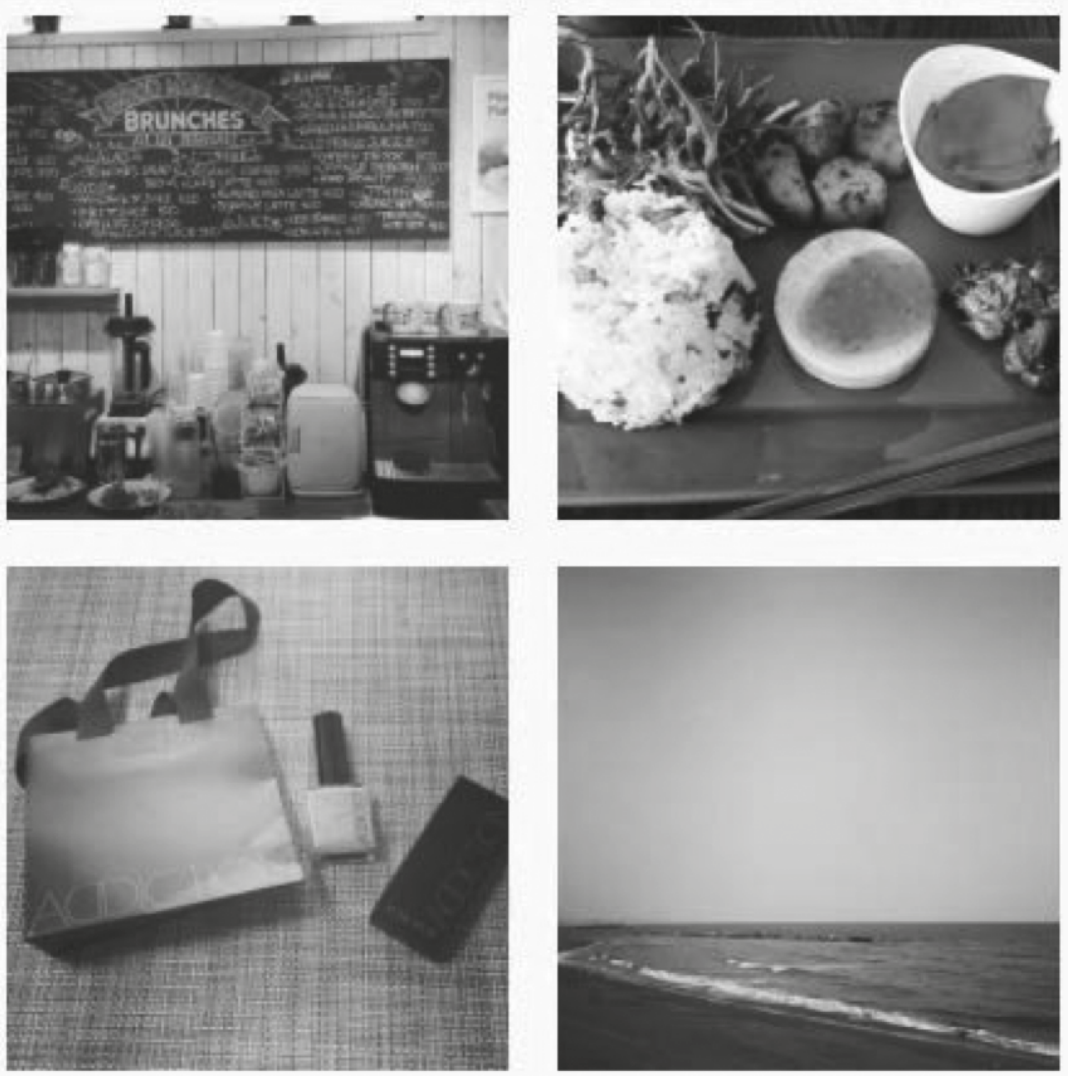

Figure 10.1: Rika's Instagram

This is reflected in her Facebook feed which is full of the pictures of the baby instead of the pictures of travel of her former life.

Soon after the birth, she started to upload pictures of her baby and new experiences of motherhood, cataloguing everything from the baby's growth record to her anxieties around raising a child. However, after a few months, she stopped sharing her stories about nurturing on Facebook. As Rika noted, "It was because I was tired of being advised by my friends who gave birth earlier than me or comparing the situation of my friends and that of me." At the time of our research she preferred to use Instagram rather than Facebook. On Instagram she shared pictures of her favorite cafe or scenery that she had found in a seaside town where she moved after marriage. She usually used a hashtag with the phrase "hope to connect with people who love the sea" which spoke to her desire to find her "long-lost self after becoming mother." 
As we see through our description of Rika's experiences, studying media use over time allows us to understand the dynamics of media practice. Over the three years Rika moved from using social mobile media to help keep a friendly eye on her mum (and vice versa) to then becoming a mother herself. This transition in life stages has had a big impact on how and why Rika uses social mobile media. Rika's motivations of media use are embedded in her strategies for intergenerational care at a distance.

Understanding these motivations, and how we might best chart them as part of broader social and cultural practices, has been a key focus of this research project. In this book we have brought together the various intergenerational and cross-cultural case studies from Shanghai, Tokyo and Melbourne over three years to demonstrate the ways in which discontinuities and continuities emerge in and around kinship in ways that are digital and non-digital, playful and non-playful, visual and tacit, quotidian and speculative. In this conclusion we summarize some of the key findings of our research, alongside provocations for the future of social mobile media, especially in context of the families and their ongoing use of digital media for intimate forms of care and co-presence.

\section{Continuity and Discontinuity}

In this book we worked to reconcile and recalibrate the often-paradoxical relationship formed in and around continuity (kinship) and discontinuity (digital disruption). Digital Kinship allows us to think through the digital historically, conceptually, and methodologically, enabling a holistic approach when contemplating our connective and empowering digital future. Throughout the four sections-Digital Kinship, Playful Kinship, Visualizing Kinship and Co-futuring Kinship-we explored different formations and manifestations of kinship within contemporary everyday media practice.

The section on Digital Kinship included two chapters that investigated the role of the different social mobile media platforms that families use to engage one another and how they are shaping — and are shaped by - familial modes of co-present intimacy. We also demonstrated how locative media use and non-use (Baumer et al. 2015; Baumer, Adams and Khovanskaya 2013; Satchell and Dourish 2009) is complicating discourses and ideas of surveillance, where surveillance is much more part of the enduring relationships to community, gossip and the tyranny of normativity in small towns and other local settings (e.g. Miller et al. 2016; Horst and Miller 2012). In 
such contexts, cultural variances around what it means to watch, and be watched, in different places, communities and families determines the tone and tenor of surveillance.

Increasingly we are witnessing different forms of informal surveillance practices emerging that require new forms of understanding and care. We focused on the notion of careful and friendly surveillance to highlight some of the ways in which familial care at a distance plays out in culturally specific ways. Emergent literature in this area suggests that, as Benjamin Burrows argues, there are new forms of relationality between the apps economy and mobile parenting (2017). As Tama Leaver and others identify, the online documenting of young children's lives by parents has created implications and ramifications we are still yet to fully understand (2017).

In Section II, Playful Kinship, we began with a chapter on digital gifts and rituals, which revisited the enduring discussions around mobile media as gift and the important anthropological meanings of the gift as a practice that both sheds light on and reinforces power relations. It highlights how digital gifts-often-playful forms of emojis and other cute images of animals, places and faces-also include locative information that is designed to share as well as obfuscate, as people attempt to keep while giving. Following earlier sociologists, boyd (2014) has also described this practice among teens as social stenography. Chapter 5 then turns to the ways in which people "play" with co-presence through different engagements with digital media. This chapter additionally reflects upon methods developed in the field to negotiate new forms of knowing — especially in terms of the emergent field of haptic media studies.

Section III, Visualizing Kinship, engages with the "visual turn" that dominates the use of social mobile media. Increasingly, visual apps like Instagram are being deployed to create new forms of ambient and copresent intimacies. However, against the weight of the digital archive of the everyday, some participants are choosing not to share as a new way in which to give different, sometimes material, meanings to images as part of digital wayfaring processes.

In the concluding section IV, Co-futuring Kinship, we explore our participants' emerging practices and perceptions around self-tracking and co-social tracking for familial care at a distance. We reflect upon some of the emerging concerns around informal digital health in which our older adult participants are keen to ensure that the technological (i.e. mobile app as silver bullet) doesn't replace face-to-face interaction. We also consider the role of datafication in terms of digital legacy. 


\section{Implications for Digital Media Practices in Households}

In this final section we conclude by reflecting upon the implications of this cross-cultural study for the future study of social mobile media. We believe that this is a first exploration - with many more intriguing possibilities with different cultural contexts. This is in many ways the tip of the iceberg with respect to what we learn from attending to kinship and familial dynamics given that much of the current literature on locative media, as part of social mobile, tends to focus upon the discursive dimensions of surveillance in western countries or the platforms that make surveillance and knowledge of location possible. These final thoughts are as much summaries of the findings of our work as they are provocations for the future of research.

Firstly, there is a tremendous need for methodological innovation and intervention when it comes to studying locative media as part of social mobile media practice. Much of the literature around locative media doesn't study it over time, over cultures, over changes. While behavior can be tracked, mapped or visualized on apps, the interpretation of and motivations for practices are difficult to study in depth. It also misses out on the experiences of social mobile media use. As Chapter 5's discussion of the emergence of tactile digital ethnography suggests, attention to the haptic dimensions of locative media apps develops an understanding of how locative media have become intertwined in the routines and habits of everyday life.

This discussion also enables us to understand the ways in which these habits, practices, and knowledge merge and evolve into our embodied knowledge, much like riding a bike, walking or eating. How does the knowing hand communicate different forms of embodied experiences that can teach us as researchers? There remains a great deal of work to be done in this area of media studies. There are also serious ethical issues to consider. For example, as researchers, we need to develop-in co-design with participants-iterative and dynamic methodological innovations, and thoughtful interventions that enable new ways to understand how locative media is being used in everyday life, with particular attention to changes over time.

We also believe this study highlights the need fornew theories and language on surveillance as an iterative and social practice. For most of the participants in our Melbourne study, privacy — with a Capital P—was an abstract concept associated with transnational corporations and the government who might mine their data for profit, information and so on. Yet privacy was of 
paramount concern. The privacy that played out in a tangible way for the individuals and the families in our study was the monitoring and surveillance of people they knew. We see in previous work by Alice Marwick (2012) that the social is a key dimension of surveillance. Through the examples of notions and practices of surveillance across three different contexts, our study highlights the importance of more complex, dynamic and micro understandings of the relationship between media and sociality. How can the knowledge generated from the field be translated into ways in which we articulate different types of what we have called in this book "friendly surveillance"?

At the same time, we do recognize the broader power structures that shape the platforms we have at our disposal, as well as the access we (and others) have to our own content creation. Much of the default use of geo-tagging in early camera phone apps was, for participants, about a type of archival process (as noted in Chapter 6). However, with the rise of spontaneous image apps like Snapchat, and concerns around the corporatization of data and digital legacy issues, participants are starting to not share.

There are also indications that with the widespread popularity of particular platforms, people are also withdrawing to find alternative spaces for sharing and navigating particular platforms for specific practices, as much as for their affordances as the social connections and disconnections that entangle and accompany them. This phenomenon goes against much of the earlier literature of camera phone apps as part of networked visuality. Studying this phenomenon for researchers presents challenges, as it can't be sourced online and involves deploying ethnographic inquiry into motivations for non-use. Understanding the various notions of privacy and surveillance being used by participants - and at specific points in their lives - helps to distinguish whether these practices of non-sharing represent a resistance to making the ephemeral part of a corporate archive, a return to esoteric or exclusive spaces of participation, or if there are other unanticipated explanations at play.

This study makes inroads into understanding how the datafication is creating paradoxical ways of being in the world. For our participants, while some enjoyed the aspects of self-care afforded by self-tracking fitness apps, others were concerned about where this data would end up. With the fallout of the Cambridge Analytica debacle, participants were increasingly concerned how their data could be manipulated for non-ethical means. And moreover, what is the life of data once someone dies? And how does this matter get addressed by the family? 
Finally, we believe that this study contributes to the understanding of families - collocated, proximate, separated or transnational-across different cultural contexts. Although we are clearly aware of the limitations of claiming broad societal shifts from studying 36 families across three different cultural contexts, the practices witnessed across multiple contex ts clearly highlights a series of intimate and domestic practices worth paying attention to in the future. As explored in the book, one of the key ways for negotiating distance is through the playful articulations of paralinguistics - emojis, stickers and stamps - that have become the fodder of daily conversations and activities. We see the need for more to be done in this area to understand the complex ways in which affective and emotional labor mediates the practices of co-presence and co-location we see emerging in this study.

There are also interesting questions being raised about the prominence of the nuclear family and the various ways in which this is being reconfigured in places like Melbourne, Shanghai and Tokyo. While there is a greater acceptance of non- dominant families (e.g. single parent, LGBT), we also see social and locative media playing an important role in grounding identity for these newly configured families. Of interest here is the fact that in the past the internet enabled spaces of communication and connection for those who did not find fit within their given families and place-based identities (e.g. see Mary Gray's work on teens in rural America); for families in Melbourne trying to make a go at creating a new sense of family, social and locative media matters a great deal.

However, as we see in one of the families we worked with — such as 30-year-old Amanda and Nerida - these spaces are as much places for identity affirmation, as they are ones that must be constantly nurtured, curated and cultivated in order to keep these spaces supportive. Sharing location, the use of paralinguistics and maintaining a sense of a family through micro-coordination all contribute to this aim. However, this is a very different project from the maintenance of connection between extended transnational families like those we see in Melbourne for whom their key relationships are, largely, normative and thus not under surveillance by the broader society in the same fashion. These variations, even within cultural contexts, make claims about what families do and not do difficult to generalize. It is clear that locative and social media will play a role in all of these family's lives, yet it is precisely those differences that we believe matters for any future theoretical development of the field.

More work needs to be conducted around the mundane uses of locative media especially in terms of the relationship in and hook-up apps. As 
hook-up apps become the norm for younger generations there is a need to understand the various ways in which gender, sexuality, class and ethnicity inform use and non-use. Currently much of the work around hook-up apps has focused upon use by male GLBT users (Mowlabocus 2010; Blackwell, Birnholtz and Abbott 2014; Brubaker, Ananny and Crawford 2014), while straight and queer women users and non-users have been overlooked (Murray and Anderson 2016; Tang 2015; Albury and Byron 2016). Mapping this movement of use - and non-use - over time as a dynamic practice will teach us much about the power of locative media for mundane intimacy.

We therefore conclude this book by calling for work that pushes us to challenge and contest our concepts such as friendly surveillance, the intimate mundane or even Digital Kinship in order to further develop the field of locative media studies.

\section{References}

Albury, Kath and Paul Byron. 2016. "Safe on My Phone? Same-Sex Attracted Young People's Negotiations of Intimacy, Visibility, and Risk on Digital Hook-Up Apps." Social Media + Society October-December 2016: 1-10. DOI: 10.1177/2056305116672887.

Blackwell, Courtney, Jeremy Birnholtz and Charles Abbott. 2014. "Co-situation and impression formation using Grindr, a location-aware gay dating app." New Media \& Society 17 (7):1117-1136. DOI: 10.1177/1461444814521595

boyd, danah. 2014. It's complicated: The social lives of networked teens. New Haven: Yale University Press.

Brubaker, Jed, Mike Anani and Kate Crawford. 2014. "Departing Glances: a sociotechnical account of 'leaving' Grindr." New Media \& Society 18 (3): 373-390. 10.1177/1461444814542311.

Burrows, Benjamin. 2017. "YouTube Kids: The App Economy and Mobile Parenting." Social Media \& Society. April-June 2017: 1-8. DOI: 10.1177/2056305117707189.

Gray, Mary. 2009. Out in the Country: Youth, Media, and Queer Visibility in Rural America. New York: New York University Press.

Horst, Heather, and Daniel Miller. 2012. "Normativity and materiality: A view from digital anthropology." Media International Australia 145 (1): 103-111.

Leaver, Tama. 2017. "Intimate Surveillance: Normalizing Parental Monitoring and Mediation of Infants Online." Social Media \& Society April-June 2017:1-8. DOI: 10.1177/2056305117707192.

Marwick, Alice E. 2012. "The public domain: Social surveillance in everyday life." Surveillance and Society 9 (4): $378-393$. 
Miller, Daniel, Elisabetta Costa, Nell Haynes, Tom McDonald, Razvan Nicolescu, Jolynna Sinanan, Juliano Spyer, Shriram Venkatraman, and Xinyuan Wang. 2016. How the world changed social media. London: UCL Press.

Mowlabocus, Sharif. 2010. Gaydar Culture: Gay Men. Technology and Embodiment in the Digital Age. Farnham: Ashgate.

Murray, Sarah and Megan Anderson. 2016. "Lez takes time: designing lesbian contact in geosocial networking apps." Critical Studies in Media Communication 33 (1): 53-69. DOI: 0.1080/15295036.2015.1133921.

Tang, Denise. T. S. 2015. "Essential labels? Gender identity Politics on Hong Kong Lesbian Mobile Phone Application Butterfly." In The Routledge Handbook of New Media in Asia, edited by Larissa Hjorth and Olivia Khoo, 263-274. London: Routledge. 



\section{Author Biographies}

Distinguished Professor Larissa Hjorth is an artist and digital ethnographer. Hjorth has two decades experience working in cross-cultural, interdisciplinary, collaborative creative practice and socially innovative digital media research. Hjorth is currently the Design \& Creative Practice ECP Platform Director at RMIT University and visiting Professor at University of Osaka co*design center. Since 2000, Hjorth has been researching the socio-cultural dimensions of mobile media and gaming cultures in the Asia-Pacific - these studies are outlined in her books such as Mobile Media in the Asia-Pacific (Routledge, 2009), Games \& Gaming (Berg, 2010), Online@ AsiaPacific (with Arnold, Routledge, 2013), Understanding Social Media (with Hinton, Sage, 2013), Gaming in Social, Locative and Mobile Media (with Richardson, Palgrave, 2014), Digital Ethnography (with Pink, Horst, Postill, Lewis and Tacchi (Sage, 2016), Screen Ecologies (with Pink, Sharp and Williams, MIT Press, 2016) and Haunting Hands (with K. Cumiskey, Oxford Uni Press, 2016).

Kana Ohashi is a postdoc fellow at the Graduate School of Media and Governance, Keio University, Japan. Her PhD is from Keio University and she studied Documentary Film making at MET FILM SCHOOL. Her research interests: mobilities studies, sociology of families, and video ethnography. She is especially interested in how people experience migration and how people maintain long-distance relationships with their family members. Currently she is making ethnographic videos on transnational lives in Japan.

Jolynna Sinanan is a senior research fellow at in the School of Media and Communication at University of Sydney, Australia. Prior to this post, she was a VC postdoc at RMIT University, and before that she was a Research Fellow in Anthropology at University College London with the Why We Post project, funded by the European Research Council, which compared uses of social media across eight countries. She is currently finishing a book from the study, Social Media in Trinidad (UCL Press) and is the co-author of Visualising Facebook (UCL Press) and Webcam (Polity) with Daniel Miller. 
Sarah Pink is Professor and Director of the emerging technologies lab at Monash University, Australia. She is Visiting Professor at Halmstad University, Sweden and Loughborough University, UK, and Guest Professor at Free University, Berlin, Germany. Her recent works are usually collaborative and include the books Theoretical Scholarship and Applied Practice (2017), Digital Materialities (2016), Digital Ethnography: principles and practice (2016), Screen Ecologies (2016), Doing Sensory Ethnography 2nd edition (2015), and Media, Anthropology and Public Engagement (2105). Less conventional works include Un/Certainty iBook (2015), Laundry Lives documentary film (2015) and the Energy and Digital Living website www.energyanddigitalliving.com.

Heather Horst is Professor in the School of Media and Communication at University of Sydney, Australia. Her research focuses upon understanding how digital technologies, mobile phones and other forms of material culture mediate relationships, learning, and mobility. These themes are reflected in her publications which include The Cell Phone: An Anthropology of Communication (Horst and Miller 2006, Berg), Hanging Out, Messing Around and Geeking Out: Kids Living and Learning with Digital Media (Ito, et al. 2010, The MIT Press), Digital Anthropology (Horst and Miller Eds 2013, Berg), Digital Ethnography: Principles and Practices (Pink, Horst, et al, 2016, Sage), The Routledge Companion of Digital Ethnography (Hjorth, Horst, Galloway and Bell Eds, 2016, Routledge) and Location Technologies in International Context (Wilken, Goggin and Horst, eds. 2017, Routledge). Her current research, funded by the Australian Research Council, the European Union Horizon 20/20 Program and industry partners, explores transformations in the mobile telecommunications industry and the emergence of mobile, social and locative media practices across the Asia-Pacific region.

Fumitoshi Kato (Ph.D., Communication) is currently working as a Professor at the Faculty of Environment and Information Studies, Keio University, Japan. His research interests include: communication theory, media studies, socio-cultural impacts of new technologies, qualitative research methods. He is especially interested in the use of camera phones in the context of place-making and community development. For the past ten years, he has been conducting field research in various local communities in Japan, with a primary focus on the notion of "mobile learning." 
Baohua Zhou is a Professor and Assistant Dean at the School of Journalism, Fudan University. He is director of the new media communication master program and associate director of Media and Public Opinion Research Center at Fudan University. He was a visiting scholar at University of Pennsylvania. Zhou was awarded the Changjiang Young Scholar by the Ministry of Education of China and Excellent Young Scholar Award by the Chinese Association for History of Journalism and Communication. His research focuses on digital media, media effects, public opinion, and data mining. Current research projects include: social differentiation, media use, and citizenship engagement in China; ICTs and migrant workers; social media and journalism, and public opinion on social media, among others. His research has been published in Asian Journal of Communication, Chinese Journal of Communication, Communication \& Society, and various communication journals in China. 



\section{Index}

\section{Page references for figures are italicized}

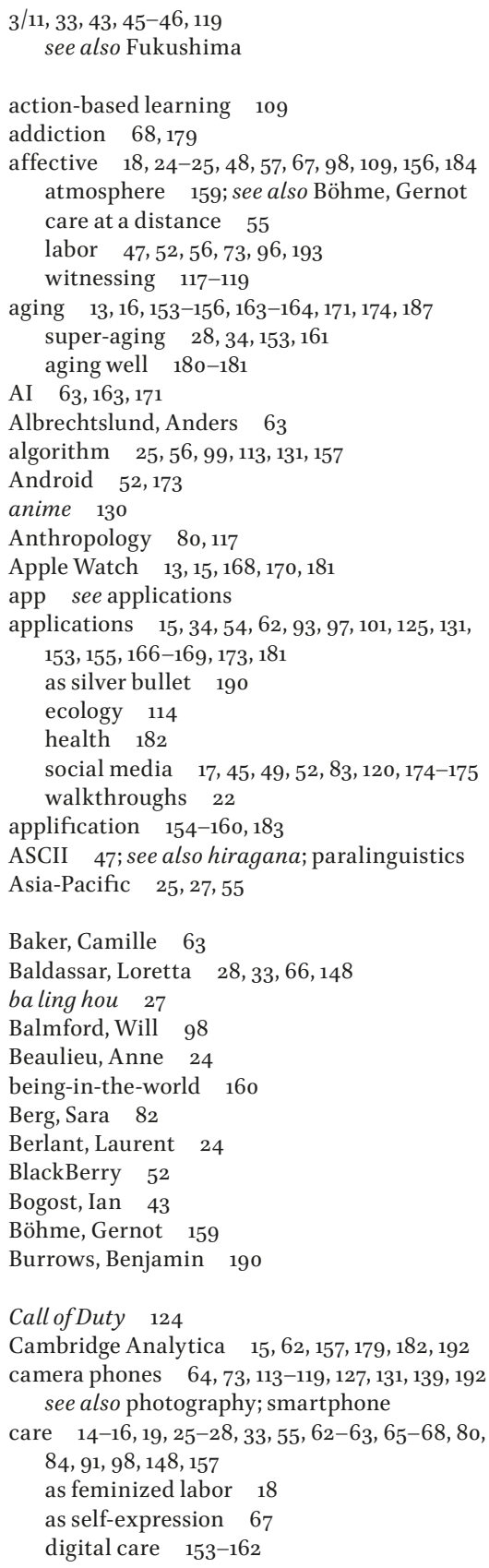

domestic $\quad 84-87$

informal $163,165^{-166,171-172,180,182}$

over-care $72-73$; see also care at a distance care at a distance $13-20,25,27,34,46-47$,

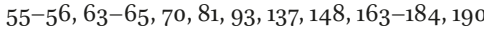

Chalfen, Richard 119

Chief Investigator 21

children $19,27-30,55^{-} 56,65,68,71-73,81,87$, 101-104, 139, 164-166, 172-174, 177, 182

Chow, Rey 96

Chunyu Doctor 173

CI see Chief Investigator

Clark, Lynn Schofield 23

co-futuring $15,34,113,153^{-154}, 161,163,165$, 171, 183, 189-190; see also kinship

co-presence $17,19,22-24,34,45,49-50,61,66$,

$79,87,90,114,117$

and play 93

creative $5^{6}$

frequency 106

gift giving rituals 34,90

intimacy $56,91,108-109$

intimate mundane $16,20,22,113$

of social media $\quad 16,119,129,131$

Crawford, Kate 56 ; \#ImageNetRoulette 63

creativity $44,99,160$

cross-cultural $14-15,22,63,130-131,189,191$

data $13,27,63,99,113,117,154-160,167-171$, $178-184,187,191-192$

Big Data $\quad 25,131,156-157$

collection and analysis $\quad 27,29$

feeling $156,15^{8}$

for care 163,187

little 157

rhythms of 99

trails $14,131,163,170,182$

transition 14

dataveillance $14,63,157,163$

datafication $13-16,35,62,113,154,156-160$, $163,165,180-183,190,192$

decomail 46

DeVault, Marjorie L., 143

di Leonardo, Micaela 66

digital health $34,63,113,153-61,169,172-175$, 190

Digital Kinship $\quad 13-23,27-28,33,45,61,73$, 8o-81, 109-110, 137-141, 143, 147, 187, 189, 194 See also kinship

digital legacy $113,130,154,158,167,170$,

$182-183,190,192$

stewarding 33

Dow Schüll, Natasha 157 
e-commerce 49,173

Elias, Ana Sofijia 180

e-mail $\quad 69,101,119$

embodiment $\quad 32,94-95,99,117,158,184$

Emojis 19, 45, 47, 54-57, 79, 96, 108-109, 190, 193

Emoticons $\quad 45-46,55,104,105,106-110$

emotion $33,46,61-62,66,183$

mediated 67

work $138,143,147$

Eng, David 23

Erickson, Erik 138

ethics 95

of media practice $\quad 15$

ethnography $13,20-21,26,154,157-158,160$ engage empathetically 21

digital $3^{2}$

tactile $22,32,34,93-95,191$

see also interviews

emplacement $114-115,131,147$

everyday $32,54,61-64,93,102,110,113-119$, $141,153,163-184,191$

biopolitics 56

observation 82

play 80

rituals 87

Facebook 17, 19, 29, 33, 43, 49-54, 51, 61-69, $89,97,101,119-124,127,138-142,158,169,171$, $179,181-182,188$

Messenger $85,108-109$

memories 113

Farman, Jason $\quad 157$

FindFace 62

Flickr 114

friendly surveillance see surveillance

Frow, John 81

Foursquare 71

Fortunati, Leopoldina $\quad 67$

Foucault, Michel 44

Fujimoto, Kenichi 45

Fukushima $33,43,119,171,183$; see also $3 / 11$

game $95^{-102,154-156,159,164,169-171}$

gamification $99,156,160$

Gawande, Atul 153

Geismar, Haidy 117

genealogy 31,44-45,109

digital 33,43

gesture $95,98-99,139$

GFW see Great Firewall

gift giving $17,19,34,79-85,86,87,90,137$

digital, 84,90

guanxi (social capital), 82

social cohesion 91

system of reciprocity 82

the Red Pocket 83

Gill, Rosalind 180

Gillespie, Tarleton 33

GLBT 28, 32, 194

Goffman, Erving $\quad 23$, 118; see also co-presence

Gómez Cruz, Edgar 141
Google Maps 93-94

Go-Pro 21

Gray, Mary 193

Great Firewall 71, 127; see also GFW; surveillance

Haldrup, Michael 143

Hannover 128

Hao Daifu 173

haptic $20,94,96-110,159-160,191$

digital media $\quad 156$

ecology 22, 95

media studies 159,190

play poetry 103

see also ethnography; mobile media; play

Harper, Richard 82

Harrisson, Tom $\quad 116$

HCI see human-computer interaction

healthcare $28,159,176,178$

self-tracking apps 15,157

Herzfeld, Michael 25

hiragana 47; see also paralinguistics

Hjorth, Larissa 44, 98

Hochschild, Arlie $\quad 18,138$

Hong Kong 176

Horst, Heather 137

human-computer interaction 174

Humphreys, Lee 25

Iijima, Katsuya $\quad 164$

impression management $32-34,113,115,118$, 122, 129; see also Goffman, Erving

Inayatullah, Sohail 153; see also co-futuring; UNESCO

information technology 64

Ingold, Tim 44

Instagram $13,19,34,45,50-51,97,114-125,120$, $127,129,140-141,160,169,180,188,190$

Instagramable 118

stories 113

intergenerational $14-22,27-31,55-57,63,71$,

$137,153^{-154}, 161,163,170,174$

care at a distance $\quad 189$

communication $45^{-47}$

data for 187

digital gifts 83

digital literacy $\quad 55,184$

informal care $\quad 165$

WeChat 173

Internet of Things 27

interviews $21,29,32,103$

face-to-face 98

snowballing 31

video 22

see also ethnography; participants

intimacy $14,23-29,33,47,61-62,72,80,96$, $98-100,141,156$

co-present $54-57,69,87-91,113-115,129$, $131-132,189$

cultural 24, 73

digital gifting 90 
during times of grief $\quad 46$

mobile 25

mundane $120,145,194$

sharing 139

IoT see Internet of Things

iPad 93-94, 94

iPhone $\quad 5^{2}, 89,97,108,169$

Ishiguro, Hiroshi 171

Ito, Mimi 23

Iwasaki, Masanori 164

Jamieson, Lynn 23

Jennings, Humphrey 116

Jiepang.com 71

Jin, Xing 173

Johns, Nicholas A., 114

juku 16

Kaixin.com $\quad 127$

Kato, Fumitoshi 116

kawaii (cute), 46-48, 55, 79, 109

keitai 16, 19, 31, 47; see also mobile phone

Kelly, Kevin 157

Keogh, Brendan 95

kinship $14-15,82,187,189,191$

boundaries of 57

co-futuring $33,153,163-165,171,183$

playful $73,80,84,190$

rituals 49

visualizing $113^{-115}, 131$; see also Digital

Kinship

kinwork 66, 148

Kiwaki, Nachiko $\quad 164$

kodoku-shi 164

koritsu-shi 164

Kücklich, Julian 56; see also Playbour

kyaraben 130

labor $160,47,57$

affective $\quad 47,52,56,73,96$;

and care 148

emotional 18, 138, 193, see also Hochschild, Arlie

feminized form $\quad 18$

as global political economy $\quad 56$

playbour $5^{6}$

lamarre, Thomas 33

see also platformativity

laptop $54,66,179$

Larsen, Jonas 143

Lasén, Amparo 67

Leaver, Tama $\quad 19,167,190$

Lego 124

Licoppe, Christian 114

LINE $15,18,20,68-70,70,79,86,89,97,102$,

104,171

as digital genealogy 31

familial use $\quad 101,119-123,139-140,165^{-169}$ post 3/11, 45-48; see also 3/11; Fukoshima stamps \& stickers $19,20,45,47,80$

Ling, Richard 84 locative media $\quad 15,21-27,57,80,90,96,131,160$, $174,183,191$

and algorithms $\quad 25,113,131$

familial use $\quad 20,62,72,87,94-95,109$, $114-115,193$

use and non-use $\quad 19,32,84,93,189,194$

Lüders, Marika 25

Lupton, Deborah $\quad 14,63,157-160,183$

see also dataveillance

Madge, Charles 116

Madianou, Mirca $\quad 5^{0-51}$

Malaysia 142

Malinowski, Bronislaw 81

mapping 98, 109, 183,194

Marwick, Alice 25

Mason, Jennifer 148

Mass Observation project $\quad 116$

Massey, Doreen 147

Matsuda, Misa $16,18,96$

Mauss, Marcel 81

media practice $14,26,30-33,94,189,191$

Melanesian kula ring 80 ; see also gift giving

Melbourne 14, 21, 26, 31-32, 50-53, 65, 84, 88, $93-94,108,123,137,142,178,182,191$

memory 114, 119, 139, 171

collective $113-115,130$

memorialization 115

meshi-tero 141

Meyer, Eric T., 141

Meyrowitz, Joshua 64

Miller, Daniel $\quad 51,137$

Mitene 167

MO 117, see also Mass Observation project

mobile phone $13,17,27,48,52,72,82,139$,

147

and self-cataloguing $\quad 115$

games 155

social media $\quad 19,119,128,179$

see also smartphone; iPhone; sumaho

mobilism $\quad 45^{-46}$

mobility $24,29,33,45^{-46}, 61,72,146,155,174$

in Japan 45

studies 159

moblog 116

modes of perception $\quad 98,156$

Montfort, Nick 43

more-than-human 158-160

motherhood $66,103,126,143,146,188$

and social media $\quad 49,53,55,68-73,86,125$, 130, 139

family photography $\quad 143$

MySpace $\quad 5^{1}$

Nafus, Dawn 157

Netflix $\quad 169$

New Materialism $\quad$ 98, 159

New York 128

Neoliberalism 157

Nintendo, DS 95

Wii 95, 99 
Nonoyama, Hisaya 29

non-sharing $113,115,130-132,137,192$

Okabe, Daisuke $\quad 23,45,96$

One-Child policy $\quad 29,72,87,171$

Paglen, Trevor 63

Paprika app 125

paralinguistics $19,45^{-47}, 51,55^{-}-57,73,166$, 193

see also hiragana; ASCII

parenting 19, 61, 87, 190

Parisi, David 95

Paterson, Mark 98; see also touch

Petersen, Søren Mørk 114

performativity 102,160

photography $113,117,124,128,137-139$

in Japanese culture 119

kyaraben 130

tourism 142-143

Photoshop 128

Pink, Sarah 32

Pinterest 124

platformativity 19, 33, 43; see also lamarre, Thomas

Platform Studies 43

playbour $\quad 56$

play $34,43,54,73,80,85,93,109-110,155$

as resistance 99

haptic 20, 96-99, 102

videogame 95

virtuoso 99

see also Pokémon GO; PokoPoko

Plummer, Ken $\quad 116$

poetry 103

Pokémon GO 155-156, 164

PokoPoko 101, 102

Pols, Jeanette $15,27,165,178$

polymedia $\quad 5^{1}$

potlach 80 ; see also gift giving

privacy $24,32,5^{0}-5^{2}, 61-63,71,98,131$,

157-158, 169, 173-179, 191-192

see also surveillance; dataveillance

QQ 29, 43, 48. 71, 104

QS, See Quantified Self

Quantified Self 14, 99, 113, 156; see also datafication

Qzone 71,127

Reading, Anna 117

Renren.com 127

reversi 169

Richardson, Ingrid 98

rituals $15,17,22,34,45,66,79-87,148,184,190$ food 118

mundane 54

of care 71

see also gift-giving

Ruppert, Evelyn 14
Samsung $5^{2}$

sensory studies $\quad 98,159$

Shanghai $14,21,27,28,49,71,83,86-88,102$, 127-130, 144, 171-178, 189

Sheller, Mimi 24

Shogi Wars 101

Sicart, Miguel 54, 73, 99, 102

Skype 108

smartphone $13,16-17,23,27,51,98,100,101$,

$116,123,142,147,169,172$

apps 166

health 182

screens 160

SMS 82; see also mobile phone; sumaho

Snapchat $113,115,122,129,131,192$

SNS 130

sociality $56,115,117,132,143,147,153,156-157$, $171,176,192$

social proprioception 157; see also Farman, Jason

social media $14,29,32,43,56,80,99,114,127$,

$148,153,171,173,193$

care 72,177

photography $117-124,139,147$

platforms $17,45,138$

surveillance $26,61,64$

Social Network Services; see SNS

Solitaire 170

Sony Walkman 96

Star Wars 124

Stark, Luke 56

storytelling 157

Strava 157

STS 171

sumaho $16,19,31,48,98,119,122$; see also smartphone

Sun, Jianjun 173

surveillance $16,18-20,25-33,52,63-73,154$, $158,172,189,192$

ambient 18

careful 19,65

friendly $18,57,80,93,140,194$

informal 18,190

intimate 19,167 ; see also Leaver, Tama

lateral 26,62

social 25, 61-62, 192; see also Marwick, Alice voluntary panopticon 63 ; see also care at a disance

Swarm $\quad 96-97,96$

Swick 159

tactile $\quad 22,32,94,99,160,191$

knowing hands 95

see also ethnography

Taylor, Alex S., 82

Telenoid 171

Tetris 170

tracking $13,26,154,174,183,190$

data 14,62

health $15,187,192$ 
self-tracking $14,63,113,158,172,175,182$ see also location media; Lupton, Deborah;

QS; friendly surveillance; surveillance transnationalism $31,33,61$

Tokyo $16,21,27,30-31,46,61,68-69,79,89$, $96-100,118,122,125,130,139,165,170,193$ touch $93,94,95,96-99$

travel $54,91,119,124,137-148,188$

Tsvetkov, Egor 62

TV 94, 99, 169

Twitter $\quad 45^{-46}, 97,119-122,130,140$

Uber 25

UNESCO 153

Urry, John 142

van Dijck, José 14, 44, 114

video cameras 64

Villi, Mikko 141

Vincent, Jane 67

visuality $116,119,131,137,147,184,192$

Wajcman, Judy 171

Warner Michael 24

wearables $14,62-63,117,157$

Web 2.0, 44
WeChat $15,19,29,48-73,5^{0}, 5^{0}, 71,84,85,91$, $103,105,106,107,127-129,144-147,171-178$ and Chats section $\quad 48,86,88$

Moments 32, 50,103

Red Pocket $\quad 83-84$

stickers 48, 104; see also social media; Shanghai

Weibo 29, 103, 104, 127

wellbeing 27, 153,155

WhatsApp $15,50-54,53,63,87,108,142$ group chat $52,66,180-182$

sharing $89,125^{-126}$; see also social media; Melbourne

WiFi $17,52,96$

Wii see Nintendo

Wilding, Raelene 66

Wilken, Rowan $\quad 15,52$

Whitaker, Reg 63

Wolf, Gary 157

worry about worries $\quad 73$; see also care; over-care

Xiamen $\quad 126$

Xiaofei, Zhang 173

Xiaomi 174

Yangzi, Li 173

Yongjun, Liu 173

Yttri, Birgitte 84 
